\title{
Role of p21-Activated Kinases in Pancreatic Cancer
}

\section{Dannel Yeo}

ORCID ID: orcid.org/0000-0002-6546-1209

Submitted in total fulfilment of the requirements of the degree of Doctor of Philosophy

September 2016

Department of Surgery (Austin Health)

The University of Melbourne 


\section{Abstract}

Pancreatic cancer remains one of the most lethal of all solid tumours with an overall 5-year survival rate of $7 \%$. Management has not improved significantly over the last thirty years and based on current trends, is expected to become the second leading cause of cancer-related mortality by 2030. Treatment options are limited and gemcitabine-based chemotherapy remains the standard of care as a single agent. Furthermore, the presence of the dense stroma, characteristic of pancreatic cancer, contributes to therapeutic resistance and poor therapeutic response. Thus, a better understanding of the underlying genetic and molecular mechanisms is urgently required to find targeted and effective therapies.

There is growing evidence that p21-activated kinases (PAKs) are involved in pancreatic carcinogenesis. The PAK family consist of six isoforms, two of which, PAK1 and PAK4, are upregulated and/or hyper-activated in pancreatic cancer. PAK1 can mediate many different cellular processes including the regulation of cytoskeletal dynamics and cell adhesion, the evasion of apoptosis, the promotion of cell survival, proliferation, migration and invasion, the fibrosis that constitutes the stroma, and the interplay between cancer cells and the stroma. PAK1's role has not been fully elucidated in pancreatic cancer and has not been evaluated as a target for therapeutic intervention. The work presented in this thesis investigates the role of PAK1 in pancreatic cancer and the effect of PAK1 inhibitors, alone and in combination with gemcitabine, on pancreatic cancer growth, metastasis, stroma, and survival.

First, we investigated the effect of glaucarubinone, a known inhibitor that reduces the activity of PAK1 and PAK4, on pancreatic cancer growth, migration and murine survival. Using 4 human and 2 murine pancreatic cancer cell lines, PAK1 and PAK4 was expressed in all pancreatic cancer cell lines tested and proliferation and migration/invasion inhibited by treatment of glaucarubinone with reduction in PAK1 and PAK4 activity in vitro. Synergistic inhibition was observed when combined with gemcitabine with decrease in pancreatic cancer 
proliferation in vitro, decrease in pancreatic cancer growth in human xenograft tumours in vivo, and increase in murine survival in an orthotopic immunocompetent model in vivo. This was one of the first studies that showed clinical benefit of targeting and reducing PAK1 in pancreatic cancer.

Using more direct methods of reducing PAK1 activity, shRNA knockdown systems, and a PAK1 selective inhibitor, FRAX597, were utilised. shRNA knockdown of PAK1 resulted in a reduction in pancreatic cancer cell proliferation and survival and sensitised cells to gemcitabine in vitro. PAK1 was also found to be key regulator of signalling pathways such as PI3K and HIF1 $\alpha$. FRAX597 treatment decreased pancreatic cancer cell proliferation and migration/invasion and synergised with gemcitabine to decrease cell proliferation in vitro. FRAX597, combined with gemcitabine, reduced pancreatic tumour volume and increased murine survival in preclinical orthotopic immunocompetent murine models in vivo. Although, further clinical validation is required, it illustrates the clinical potential of a PAK1 inhibitor, FRAX597, combined with gemcitabine to improve pancreatic cancer patient outcomes.

PAK1's role was investigated in pancreatic stellate cells (PSCs), which are primarily responsible for the fibrosis that constitutes the pancreatic cancer stroma. This was the first study to show the presence of PAK1 activity in isolated human PSCs. The treatment of the selective PAK1 inhibitor, FRAX597, on PSCs resulted in a reduction in their activation, proliferation, and increase in apoptosis in vitro. PAK1 knockout mice tumours had decreased expression and activity of PAK1, associated with increased murine survival, showing the effect of depleting host PAK1 in an orthotopic immunocompetent murine model in vivo. These results implicate PAK1 as a regulator of PSC activation, proliferation and apoptosis and targeting stromal PAK1 could increase therapeutic response and survival of patients with pancreatic cancer. 
Together, these results illustrate the importance of PAK1 signalling in pancreatic cancer and the possible therapeutic benefit of targeting PAK1 with gemcitabine on pancreatic cancer growth and the stroma to increase the survival of pancreatic cancer patients. 


\section{Declaration}

This is to certify that:

- the thesis comprises only my original work towards the $\mathrm{PhD}$ except where indicated in the Preface,

- due acknowledgement has been made in the text to all other material used,

- the thesis is fewer than 100,000 words in length, exclusive of tables, maps, bibliographies and appendices

Dannel Yeo

September 2016 


\section{Preface}

Chapter 3 and 4 of this thesis represents work that has been published (Yeo et al. 2014, Yeo et al. 2016, Yeo et al. 2016). The work was jointly conceived by Dr Hong He and Dr Mehrdad Nikfarjam who assisted in the initial manuscript draft and were both involved in the editing and final approval before publication. The normal immortalised human pancreatic duct epithelial (HPDE) cells used were originally derived from Dr Ming-Sound Tsao's laboratory. Glaucarubinone used in this thesis was generously supplied by Dr John A. Beutler. Some of the $\mathrm{IC}_{50}$ values described in Chapter 3 (namely Chapter 3.3.1 and 3.3.2) had been submitted as part of my Honours degree and been included for comparison. I independently carried out all in vitro and in vivo experiments described, analysed the data, scripted the initial manuscript draft and performed subsequent editing in response to supervisors' and reviewers' suggestions and comments.

Chapter 5 of this thesis represents work currently under review. I conceived the work with Dr Hong He and Dr Mehrdad Nikfarjam and I independently carried out all in vitro and in vivo experiments described including the collection of pancreatic cancer specimens from resected patients, analysed the data, scripted the initial manuscript draft and performed subsequent editing in response to supervisors' suggestions and comments. Dr Hong He and Dr Mehrdad Nikfarjam were both involved in the editing and final approval before submission. Dr Phoebe Phillips assisted in providing analysis and interpretation of the data and was involved in the editing and final approval before submission.

Therefore, I estimate that my contribution to the work presented in this thesis is greater than 85\%. I also acknowledge the NHMRC (508908 to Dr Hong He; 1020983 to Prof. Graham S Baldwin), Austin Hospital Medical Research Foundation (Dr. Mehrdad Nikfarjam), Sir Edward Dunlop Foundation Grant (Dr. Mehrdad Nikfarjam), and Pancare Foundation for funding the work described in the thesis. 


\section{Acknowledgements}

It would not have been possible to complete this doctoral degree without the support of the all the people who provided advice, guidance, motivation and friendship along the way. First and foremost, I would like to thank my supervisors, Dr Mehrdad Nikfarjam and Dr Hong He, for giving me the opportunity to conduct research into pancreatic cancer and assigning me with this fascinating project. Your guidance in the research field of both clinical and basic sciences, ability to always make time for me, patience, and ongoing support were invaluable and has provided me a solid foundation to continue my future in the scientific community. Lastly, I thank you both for your mentorship and friendship over the last few years.

I would also like to thank the department of allowing me to do research in the laboratory. Special thanks to Nhi Huynh who was always willing to spare her time to go through something with me or give me a hand in the laboratory. Also to Prof. Graham Baldwin for his invaluable advice, scientific support and guidance. Thank you to the rest of the department, past and present, animal facility, and tissue biobank for all your help and the friendships I have gained.

I would also like to thank Jane Loxton and the Australian Rotary Health for the Ian Loxton Pancreatic Cancer Research Doctor of Philosophy scholarship. Jane has become a great friend over the years and have placed the vital context of my research into this cancer which sadly took her husband.

Finally, I would like to thank my parents for their unconditional love and support throughout not only these past few years, but all my life and everything they have done for me. Also, thank you to my brother for his support and for always being there when I needed it. To all my friends, I thank you for being understanding - working around my schedule - and for your support and keeping me sane. To all the people I have mentioned or couldn't fit in, you have all touched and made an impact on me and I couldn't be where I am today without you, so I thank you. 
Table of Contents

Abstract

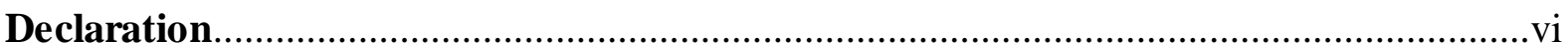

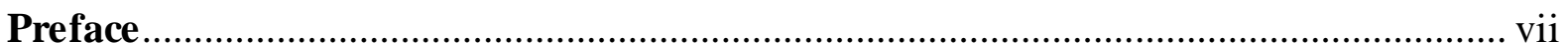

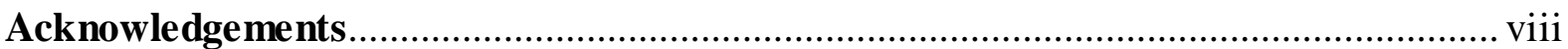

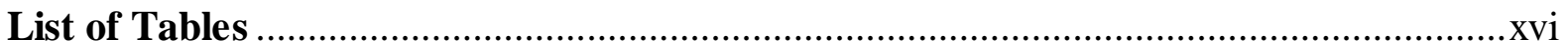

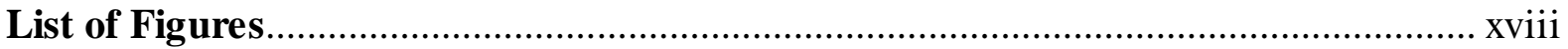

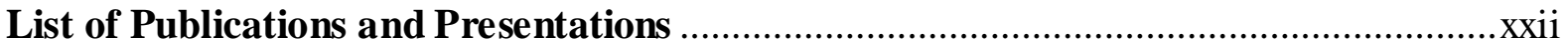

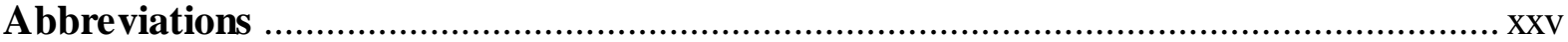

Chapter 1: Role of PAKs in Pancreatic Cancer ..........................................................

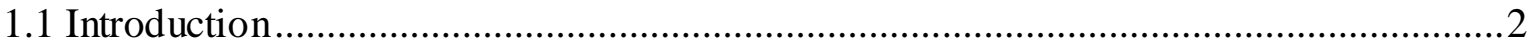

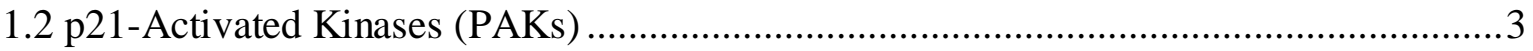

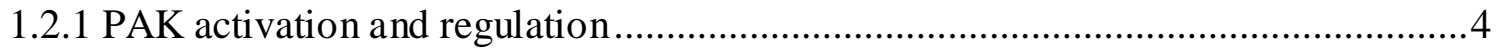

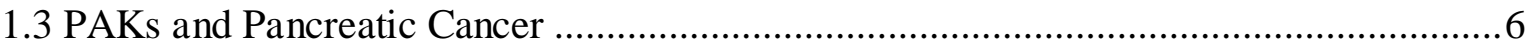

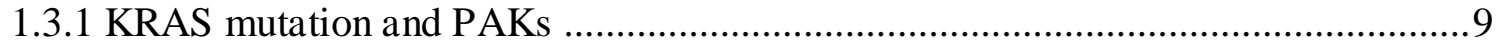

1.3.2 PAKs and Pancreatic Cancer Cell Migration and Invasion .................................11

1.3.3 PAKs, the EMT and Embryonic Pathways in Pancreatic Cancer ........................... 14

1.3.4 PAKs and Pancreatic Cancer Cell Proliferation and Survival ...............................16

1.3.5 Pancreatic Cancer Stroma and PAKs ................................................................. 19

1.3.6 PAKs, miRNAs and HSPs in Pancreatic Cancer .................................................20

1.4 Targeting PAKs as a Therapy for Pancreatic Cancer ...............................................21

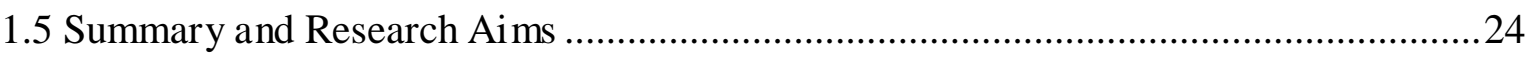




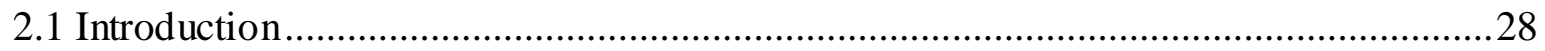

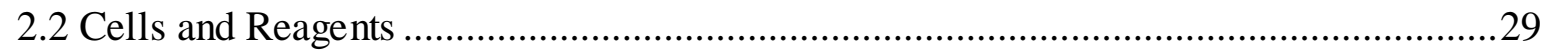

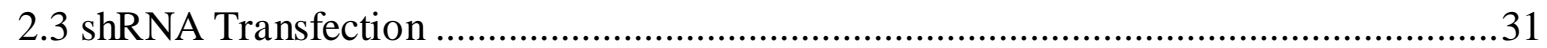

2.4 Cell Proliferation, Survival and Toxicity Assays ...................................................... 31

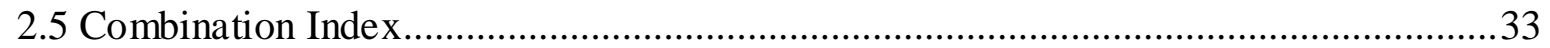

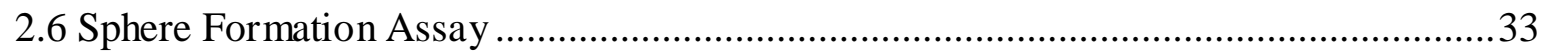

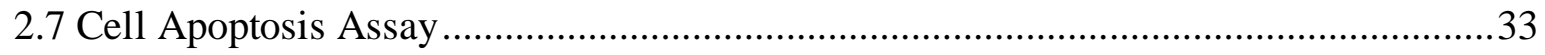

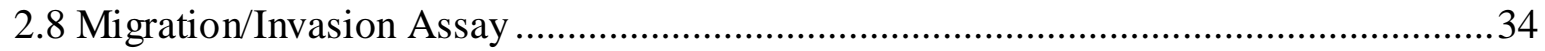

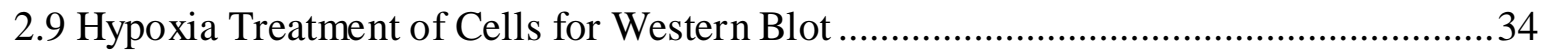

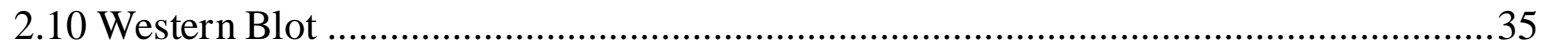

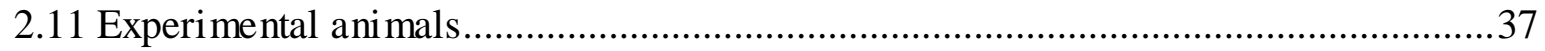

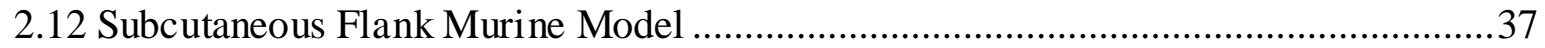

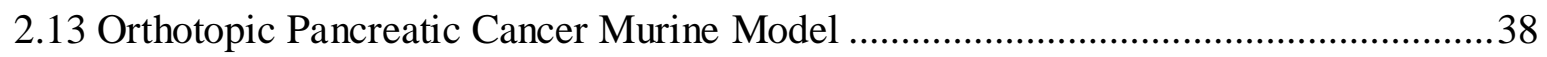

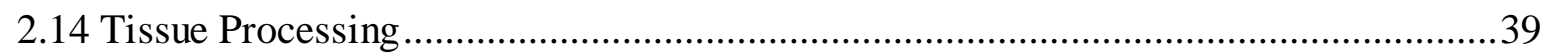

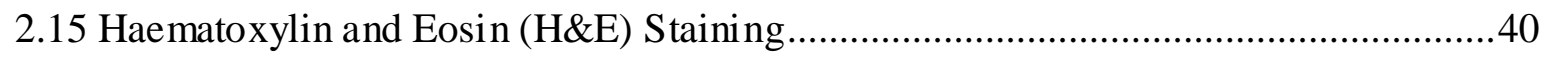

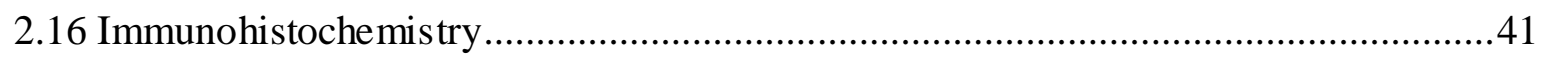

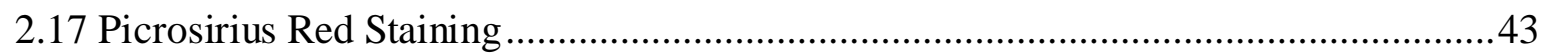

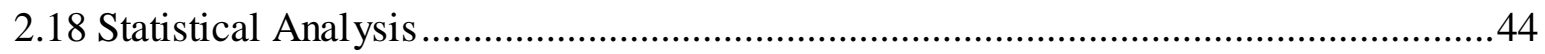

Chapter 3: Glaucarubinone Reduces Pancreatic Cancer Growth and Increases Survival

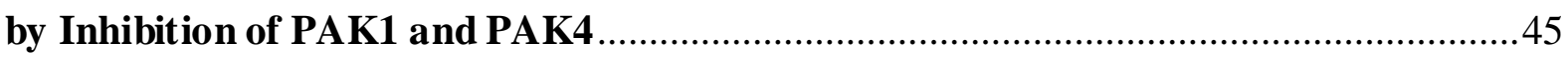

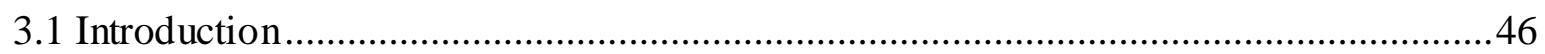


3.2.1 Subcutaneous Flank Xenograft Murine Study .48

3.2.2 Orthotopic Pancreatic Cancer Murine Study Design .48

3.3 Results 50

3.3.1 Glaucarubinone alone and gemcitabine alone inhibited pancreatic cancer cell proliferation. .50

3.3.2 Glaucarubinone inhibited pancreatic cancer cell migration/invasion .52

3.3.3 Inhibition of glaucarubinone correlated with PAK1 activity 53

3.3.4 Glaucarubinone and gemcitabine synergistically inhibited LM-P pancreatic cancer cell growth .55

3.3.5 Inhibition of pancreatic cancer cell growth by glaucarubinone and gemcitabine associated with a reduction in active PAK1 and PAK4 .56

3.3.6 Glaucarubinone and gemcitabine inhibited pancreatic cancer xenograft growth in vivo by decreasing proliferation.

3.3.7 Glaucarubinone and gemcitabine decreased the amounts of active PAK1 and PAK4 in vivo

3.3.8 Glaucarubinone and gemcitabine treatment improved survival compared to gemcitabine alone .65

3.3.9 LM-P murine pancreatic cancer cells failed to grow in $\mathrm{C} 57 \mathrm{Bl} / 6$ mice .67

3.4 Discussion .69

3.5 Conclusion .73

Chapter 4: FRAX597, a PAK1 Inhibitor, Synergistically Reduces Pancreatic Cancer Growth when Combined with Gemcitabine .74 
4.3.1 PAK1 is upregulated in human pancreatic cancer and pancreatic cancer cell lines 79 4.3.2 Inhibition of PAK1 by shRNA knockdown decreased proliferation and survival of pancreatic cancer cells

4.3.3 Inhibition of PAK1 by shRNA knockdown sensitised pancreatic cancer cells to gemcitabine .85

4.3.4 Inhibition of PAK1 by shRNA knockdown decreased clonogenic growth .87

4.3.5 Inhibition of PAK1 by shRNA knockdown reduced AKT activity and HIF-1 $\alpha$ expression .88

4.3.6 Inhibition of PAK1 by shRNA knockdown was transient in murine pancreatic cancer cell lines .92

4.3.7 FRAX597 decreased proliferation and migration/invasion in pancreatic cancer cell lines .93

4.3.8 FRAX597 and gemcitabine synergistically inhibited pancreatic cancer cell growth .95

4.3.9 FRAX597 and 5-FU synergistically inhibited MiaPaCa-2 and PANC-1 pancreatic cancer cell growth

4.3.10 Inhibition of pancreatic cancer cell growth by FRAX597 and gemcitabine is associated with reduced amounts of active PAK1 .99 
4.3.11 FRAX597 and gemcitabine inhibited pancreatic tumour growth and improved survival in orthotopic murine models 101

4.3.12 FRAX597 and gemcitabine reduced tumour growth in orthotopic murine tumours by inhibiting proliferation

4.3.13 FRAX597 and gemcitabine reduced collagen deposition in orthotopic murine tumours by decreasing activated stellate cells 105

4.4 Discussion 107

4.5 Conclusion

Chapter 5: Inhibiting PAK1 Suppresses Pancreatic Stellate Cell Activation and Increases Survival of Mice with Pancreatic Cancer. 113

5.1 Introduction 114

5.2 Methods 118

5.2.1 Cells 118

5.2.2 Cell Apoptosis Assay 118

5.2.3 Cell Migration/Invasion Co-culture

5.2.4 Cell Proliferation using conditioned media

5.2.5 Orthotopic Pancreatic Cancer Murine Study Design.

5.2.6 Subcutaneous Flank Pancreatic Cancer Murine Study 120

5.3 Results

5.3.1 PAK1 is expressed in pancreatic cancer stroma and in isolated pancreatic stellate cells 
5.3.2 Inhibition of PAK1 by FRAX597 inhibited stellate cell activation, function and proliferation, and increased apoptosis.

5.3.3 Hypoxia increased expression and activity of PAK1 in PSCs 126

5.3.4 Inhibition of PAK1 by shRNA knockdown was unsuccessful in hPSC1

5.3.5 PAK1 knockout quiescent murine pancreatic stellate cells had reduced proliferation 128

5.3.6 PAK1 knockout mice had increased survival in an orthotopic murine pancreatic cancer model 130

5.3.7 PAK1 knockout mice had transient reduction in tumour growth

5.3.8 Reduced PAK1 in pancreatic cancer cells by shRNA knockdown negatively regulated PSC and PSC proliferation.

5.3.9 Inhibition of PAK1 in pancreatic tumour cells by shRNA knockdown reduced PSCdriven migration/invasion

5.4 Discussion 139

5.5 Conclusion 144

Chapter 6: General Discussion and Future Directions 145

6.1 PAK1 plays an important role in pancreatic cancer. 146

6.2 PAK1 inhibition combined with gemcitabine improved survival and decrease tumour growth in murine models 148

6.3 Limitations and future directions 149

6.4 Conclusion 154

References 155 
Appendix i. Mouse clinical signs severity score..... 


\section{List of Tables}

\section{Chapter 2}

Table 2.10: Primary Antibodies used for Western Blotting.............................36

Table 2.16: Primary Antibodies used for Immunohistochemistry. .43

\section{Chapter 3}

Table 3.3.1: Proliferation $\mathrm{IC}_{50}$ values for glaucarubinone and gemcitabine. .52

Table 3.3.2: Migration and invasion $\mathrm{IC}_{50}$ values for glaucarubinone .53

Table 3.3.8: Cox regression survival analysis of treatment of glaucarubinone and gemcitabine in a mouse orthotopic pancreatic cancer model.

\section{Chapter 4}

Table 4.3.2: PAK1 knockdown (KD) inhibited cell growth. .85

Table 4.3.3: PAK1 knockdown (KD) decreased gemcitabine $\mathrm{IC}_{50}$

Table 4.3.7: Proliferation and migration/invasion $\mathrm{IC}_{50}$ values for FRAX597. .95

Table 4.3.9: Proliferation $\mathrm{IC}_{50}$ values for 5-FU. .99

Table 4.3.11: Cox regression survival analysis of treatment of FRAX597 and gemcitabine in a mouse orthotopic pancreatic cancer model .103

\section{Chapter 5}

Table 5.3.6: Cox regression survival analysis of PAK1 knockout $\left(\mathrm{PAK} 1^{-/}\right.$) mice and wild type (WT) mice in an orthotopic murine pancreatic cancer model. 132 
Table 5.3.8: PAK1 knockdown (KD) conditioned media inhibited pancreatic stellate cell

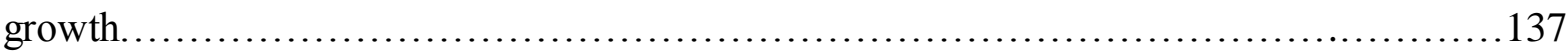




\section{List of Figures}

\section{Chapter 1}

Figure 1.2 Comparison of the structure and activation of Group 1 and Group 2 PAKs........ 4

Figure 1.2.1 Regulation of PAKs differs between PAK groups in pancreatic cancer...........5

Figure 1.3.1 PAK1 involvement in Ras-dependent pathways.............................11

\section{Chapter 3}

Figure 3.1 Chemical structure of glaucarubinone .47

Figure 3.3.1 Glaucarubinone and gemcitabine inhibited pancreatic cancer proliferation in a dose dependent manner.

Figure 3.3.2 Glaucarubinone inhibited pancreatic cancer migration/invasion in a dose dependent manner. .52

Figure 3.3.3 PAK1 and PAK4 is expressed in all pancreatic cancer cell lines tested and glaucarubinone inhibition correlated with PAK1 activity.... .54

Figure 3.3.4 Glaucarubinone synergised with gemcitabine to inhibit LM-P proliferation.....55

Figure 3.3.5 Glaucarubinone and gemcitabine synergistically reduced the amounts of active PAK1 and PAK4 in vitro .58

Figure 3.3.6 Glaucarubinone and gemcitabine synergistically inhibited pancreatic cancer tumour growth in vivo by decreasing proliferation 61

Figure 3.3.7 Glaucarubinone and gemcitabine synergistically reduced the amounts of active PAK1 and PAK4 in vivo. . .64 
Figure 3.3.8. Glaucarubinone combined with gemcitabine significantly improved survival in a syngeneic orthotopic murine model. .66

Figure 3.3.9 LM-P tumours failed to grow in C57B1/6 mice but grew in SCID mice. .68

\section{Chapter 4}

Figure 4.1 Chemical structure of FRAX597 .75

Figure 4.3.1 PAK1 is upregulated in pancreatic cancer specimens and pancreatic cancer cell lines. ... .80

Figure 4.3.2 PAK1 shRNA knockdown (KD) was specific to PAK1 and PAK1 KD reduced cell proliferation and survival .83

Figure 4.3.3 PAK1 knockdown (KD) increased gemcitabine sensitivity .86

Figure 4.3.4 PAK1 knockdown (KD) cells had decreased sphere formation .88

Figure 4.3.5 PAK1 knockdown (KD) inhibited AKT activity and HIF1 $\alpha$ expression. .90

Figure 4.3.6 PAK1 knockdown (KD) of murine pancreatic cancer cells was transient .93

Figure 4.3.7 FRAX597 inhibited pancreatic cancer proliferation, migration/invasion and survival in a dose dependent manner. .94

Figure 4.3.8 FRAX597 synergises with gemcitabine to inhibit pancreatic cancer proliferation..... .96

Figure 4.3.9 FRAX597 synergises with 5-FU to inhibit pancreatic cancer proliferation......98

Figure 4.3.10 FRAX597 and gemcitabine reduced PAK1 activity. 100 
Figure 4.3.11 FRAX597 combined with gemcitabine inhibited tumour volume and increased survival in orthotopic murine models

Figure 4.3.12 FRAX587 and gemcitabine inhibited tumour proliferation 104

Figure 4.3.13 FRAX587 and gemcitabine reduced collagen deposition by inactivating stellate cells. 105

\section{Chapter 5}

Figure 5.1 Simplified schematic diagram of the bidirectional interaction between pancreatic stellate cells and pancreatic tumour cells.

Figure 5.3.1 PAK1 is expressed in the stroma of human pancreatic cancer specimens and in isolated pancreatic stellate cells

Figure 5.3.2 The group 1 PAK inhibitor, FRAX597, decreased PAK1 activity and expression and PSC activation and function, inhibited PSC proliferation, and increased apoptosis

Figure 5.3.3 Hypoxia increased expression and activity of PAK1 and FRAX597 decreased the expression of pPAK1, $\alpha$ SMA and HIF $1 \alpha$

Figure 5.3.4 PAK1 knockdown (KD) of pancreatic stellate cells was unsuccessful 128

Figure 5.3.5 Isolated PSCs from PAK1 KO mice had reduced cell growth....

Figure 5.3.6 PAK1 KO mice had increased survival with reduced PAK1 expression in pancreatic tumours compared to PAK1 WT mice

Figure 5.3.7 PAK1 KO mice reduced tumour growth but was intermittent

Figure 5.3.8 PAK1 knockdown in pancreatic cancer cells negatively regulated PSCs and PSC proliferation 136 
Figure 5.3.9 Tumour migration/invasion was increased in the presence of PSCs and PSCs mediated tumour migration/invasion involved PAK1 pathways ........................13 


\section{List of Publications and Presentations}

Publications:

Nikfarjam M, Yeo D, He H, Baldwin G, Fifis T, Costa P, Tan B, Yang E, Wen SW, and

Christophi C. Comparison of Two Syngeneic Orthotopic Murine Models of Pancreatic Adenocarcinoma. Journal of Investigative Surgery. 26: 352-359, 2013.

Yeo D, Huynh N, Beutler JA, Christophi C, Shulkes A, Baldwin GS, Nikfarjam M, and He H. Glaucarubinone and Gemcitabine Synergistically Reduce Pancreatic Cancer Growth via Down-Regulation of P21-Activated Kinases. Cancer Letters. 346: 264-272, 2014.

Yeo D, He H, Baldwin GS, and Nikfarjam M. The role of p21-activated kinases in pancreatic cancer. Pancreas. 44: 363-369, 2015.

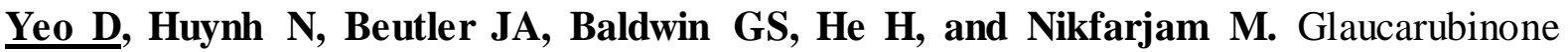
Combined with Gemcitabine Improves Pancreatic Cancer Survival in an Immunocompetent Orthotopic Murine Model. Journal of Investigative Surgery. 1-7, 2016.

Yeo D, He H, Patel O, Lowy AM, Baldwin GS, and Nikfarjam M. FRAX597, a PAK1 inhibitor, synergistically reduces pancreatic cancer growth when combined with gemcitabine. BMC Cancer. 16: 1-12, 2016.

Yeo D, Phillips P, Baldwin GS, He H, and Nikfarjam M. Inhibiting p21-activated Kinase 1 Suppresses Pancreatic Stellate Cell Activation and Increases Survival of Mice with Pancreatic Cancer. International Journal of Cancer. (under review) 


\section{Conference Abstracts:}

Oral:

Yeo D, He H, Phillips P, Baldwin G, Nikfarjam M. Targeting PAK1 in Pancreatic Stellate Cells Increases Pancreatic Cancer Survival. European Pancreas Club Meeting. Liverpool, UK. 2016

Yeo D, He H, Baldwin G, Nikfarjam M. Targeting PAK1 in Pancreatic Cancer Stellate Cells Increases Survival. Australasian Pancreatic Club Meeting. Brisbane, Australia. 2015

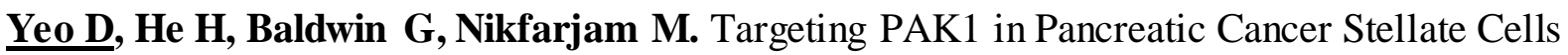
Increases Survival. Gastro 2015 GESA-AGW \& WGO International Congress. Brisbane, Australia. 2015

Yeo D, He H, Baldwin G, Nikfarjam M. A p21-Activated Kinase 1 Inhibitor, FRAX-597, Combined with Gemcitabine Synergistically Inhibits the Growth and Metastasis of Pancreatic Ductal Adenocarcinoma. AHMR Congress. Melbourne, Australia. 2014

Yeo D, He H, Baldwin G, Nikfarjam M. A p21-Activated Kinase 1 Inhibitor, FRAX-597, Combined with Gemcitabine Synergistically Inhibits the Growth and Metastasis of Pancreatic Ductal Adenocarcinoma. Biomed Link. Melbourne, Australia. 2014

Yeo D, He H, Baldwin G, Nikfarjam M. A p21-Activated Kinase 1 Inhibitor, FRAX-597, Combined with Gemcitabine Inhibits the Growth of Pancreatic Cancer. Austin Research Week. Melbourne, Australia. 2014

Yeo D, He H, Christophi C, Shulkes A, Baldwin G, Nikfarjam M. A Novel p21-Activated Kinase 1 Inhibitor, Glaucarubinone, Combined with Gemcitabine Synergistically Inhibits the Growth of Pancreatic Ductal Adenocarcinoma. Australasian Pancreatic Club Meeting. Melbourne, Australia. 2013 
Poster:

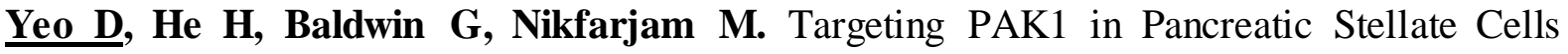
Increases Pancreatic Cancer Survival. Pancreas Club Meeting. San Diego, USA. 2016

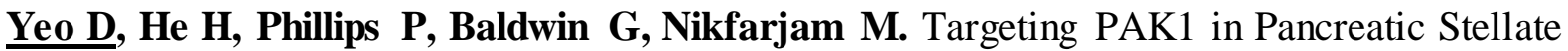
Cells Increases Pancreatic Cancer Survival. International Symposium on Pancreatic Cancer. Glasgow, Scotland. 2016

Yeo D, He H, Baldwin G, Nikfarjam M. A p21-Activated Kinase 1 Inhibitor, FRAX-597, Combined with Gemcitabine Synergistically Inhibits the Growth and Metastasis of Pancreatic Ductal Adenocarcinoma. European Pancreas Club Meeting. Toledo, Spain. 2015

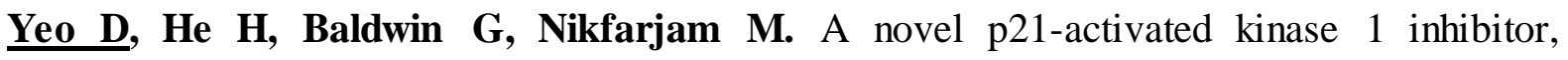
Glaucarubinone, combined with Gemcitabine synergistically inhibits the growth of pancreatic ductal adenocarcinoma. Pancreas Club Meeting. Orlando, USA. 2013 

Abbre viations
5-FU Fluorouracil
aSMA alpha-smooth muscle actin
BPE bovine pituitary extract
BSA bovine serum albumin
CAF cancer-associated fibroblast
CI Combination Index
CK19 cytokeratin 19
CT Control
DAB diaminobenzidine
DMEM Dulbecco's Modified Eagle's Medium
ECM extracellular matrix
EGF epidermal growth factor
EMT epithelial-mesenchymal transition
$\mathrm{F}_{\mathrm{a}} \quad$ fraction-affected value
FBS foetal bovine serum
FFPE formalin fixed paraffin embedded
FRAX FRAX597
G418 geneticin
Gem Gemcitabine
GFAP glial fibrillary acidic protein
GG Glaucarubinone + Gemcitabine
Glau Glaucarubinone
GPCR G protein-coupled receptor
H\&E hematoxylin and eosin
hENT1 human equilibrative nucleoside transporter 1
HGF hepatocyte growth factor
HIF-1 $\alpha$ hypoxia-induced factor 1 -alpha
HPDE human pancreatic duct epithelial
hPSC human pancreatic stellate cells 


\begin{tabular}{|c|c|}
\hline HRP & horseradish peroxidise \\
\hline i.p. & intraperitoneal \\
\hline $\mathrm{IHC}$ & immunohistochemistry \\
\hline IMEM & Iscove's modified Dulbecco's medium \\
\hline IPMN & intraductal papillary mucinous neoplasm \\
\hline $\mathrm{KD}$ & knockdown \\
\hline $\mathrm{KO}$ & knockout \\
\hline KPC & $\operatorname{Kras}^{\mathrm{G} 12 \mathrm{D}} ; \operatorname{Trp} 53^{\mathrm{R} 172 \mathrm{H}} ; \mathrm{Pdx} 1-\mathrm{Cre}$ \\
\hline miRNA & MicroRNA \\
\hline $\mathrm{NC}$ & negative control \\
\hline NF2 & neurofibromatosis type 2 \\
\hline nmPSC & normal mouse pancreatic stellate cells \\
\hline PAK & p21-activated kinase \\
\hline $\mathrm{PAK}^{-/-}$ & PAK1 Knockout \\
\hline PanIN & pancreatic intraepithelial neoplasia \\
\hline PI & propidium iodide \\
\hline PSC & pancreatic stellate cell \\
\hline PTEN & phosphatase and tensin homolog \\
\hline PUMA & p53 upregulated modulator of apoptosis \\
\hline ROS & Reactive oxygen species \\
\hline RTK & receptor tyrosine kinase \\
\hline SDS & sodium dodecyl sulphate \\
\hline shRNA & short hairpin RNA \\
\hline SNP & single nucleotide polymorphism \\
\hline TBST & tris-base saline, Tween 20 \\
\hline TGF- $\beta$ & transforming growth factor $\beta$ \\
\hline WT & wildtype \\
\hline
\end{tabular}


CHAPTER 1

Chapter 1: Role of PAKs in Pancreatic Cancer 


\section{CHAPTER 1}

\subsection{Introduction}

Pancreatic cancer is the fifth and fourth leading cause of cancer-related deaths in Australia and the United States, respectively and is expected to become the second leading cause of cancerrelated deaths by 2030, based on current management without significant improvements in treatment $(5,180)$. It is one of the most difficult human malignancies to treat with a dismal five-year survival rate of around $7 \%$ and the mortality rate almost equalling the rate of diagnosis $(79,198)$.

More than $80 \%$ of newly diagnosed patients present with advanced, metastatic cancer where curative treatments are not possible (24). Although many patients are not eligible, surgical resection remains the only treatment with curative potential. Hence, there is a reliance on therapeutics, although currently available agents are largely ineffective. As a single agent, gemcitabine-based chemotherapy continues to be the standard of care for pancreatic cancer patients although it confers only modest survival benefits with a median survival of 6.7 months $(32,185)$. There have been some recent minor advances in chemotherapeutic regimens, with the addition of nab-paclitaxel to gemcitabine, improving the median survival by roughly 2 months compared to gemcitabine alone, and the combination chemotherapy regimen of FOLFIRINOX (which is a combination of 5-FU, oxaliplatin, irinotecan, and leucovorin), improving the median survival by roughly 4 months compared to gemcitabine alone $(34,223)$. However, these chemotherapeutic agents have cytotoxic effects and their therapeutic response is often poor and chemoresistance is high (129). In order to develop more targeted and effective therapies there is an urgent need to focus on understanding the pathological mechanisms responsible for cancer development and progression at the molecular level.

The p21-activated kinases (PAKs) play a pivotal role in carcinogenesis but have not been studied extensively in pancreatic cancer. There is some evidence that PAKs play particular 


\section{CHAPTER 1}

importance in pancreatic cancer and may be a suitable therapeutic target to improve the management of patients with pancreatic cancer.

\section{2 p21-Activated Kinases (PAKs)}

PAKs were first discovered in 1994 by Manser and colleagues in a search for new proteins that interacted with small Rho-like G-proteins (139). These small Rho-like G-proteins were designated as p21 proteins, as they have a molecular weight of $21 \mathrm{kDa}$, and include proteins such as Ras, Cdc42 and Rac. Under homeostasis, PAKs play a role in many cellular processes such as cell cycle regulation, cell polarity, gene transcription and translation, and cytoskeletal reorganisation $(57,256)$. More importantly, they have been found to be upregulated or hyperactivated in a variety of human malignancies $(81,215,218)$.

Mammalian PAKs are a family of non-receptor serine/threonine kinases with six known isoforms (101). Based on their architectural similarities these isoforms can be divided into two groups: group 1 (PAKs 1-3) and group 2 (PAKs 4-6) (Figure 1.2) (90). All PAKs have a conserved C-terminal kinase domain and an N-terminal regulatory domain containing a p21binding domain (PBD), with group 1 PAKs having an additional auto-inhibitory domain (AID) and a PAK interacting exchange factor binding domain (PIX). Group 1 PAKs share a greater than $90 \%$ identity in their kinase domains and a 50\% identity with the kinase domains of group

2 PAKs. Due to the overlapping and/or redundant functions among PAK isoforms, PAK isoform-specific targeting has been challenging (53). Furthermore, multiple PAKs are often expressed in the same tissues: PAK2 is ubiquitously expressed; PAK1, PAK3 and PAK5 are detected in the brain; whereas PAK4 and PAK6 are expressed in prostate and testis; and PAK1 and PAK4 are expressed in the colon (18). PAK2 or PAK4 play a crucial role in the development of the embryo as their gene deletion results in embryonic lethality in mice (177). These observations suggest that each of the 6 PAK isoforms have different organ-specific roles. 

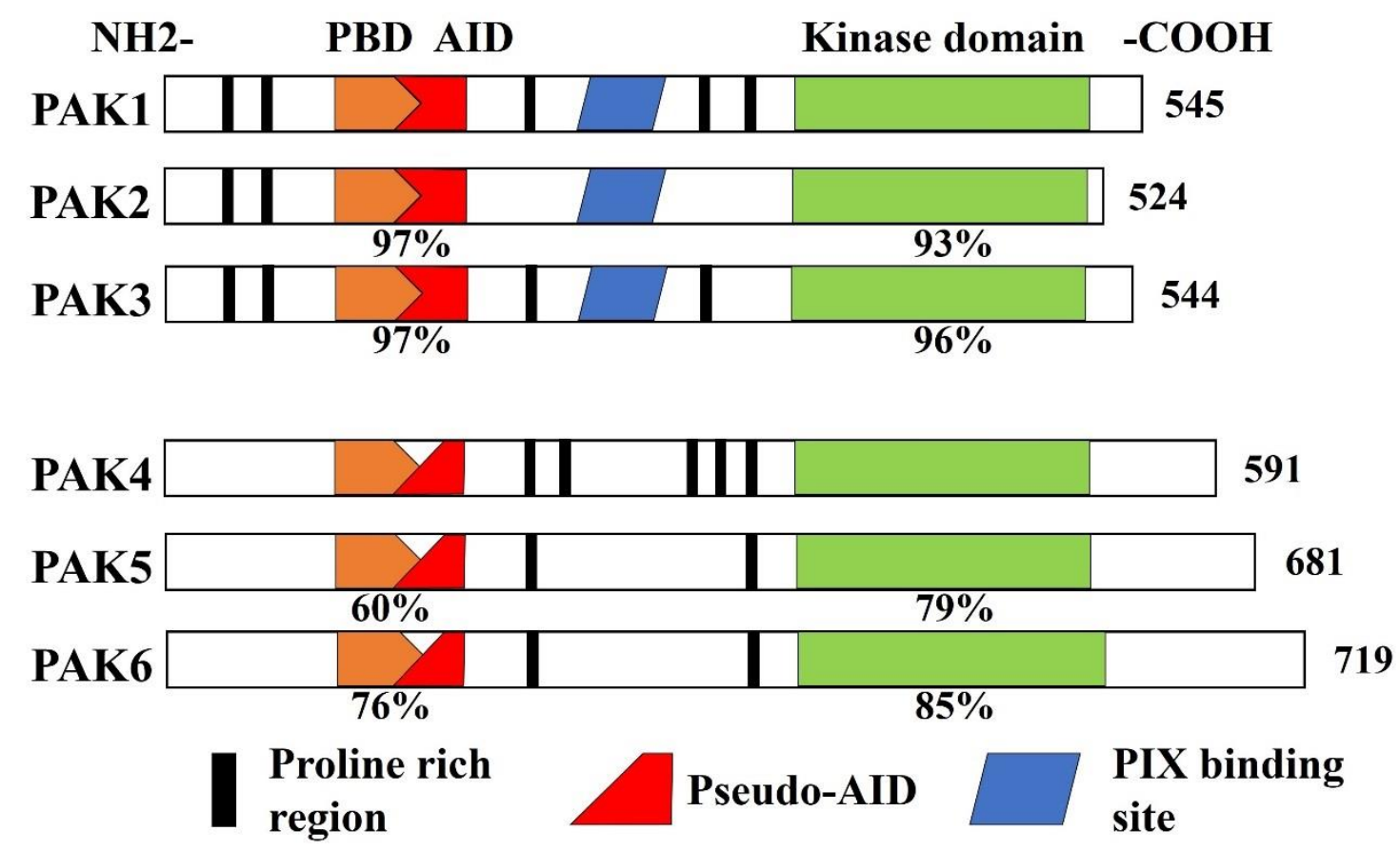

Figure 1.2 Comparison of the structure and activation of Group 1 and Group 2 PAKs. All 6 PAK isoforms have proline-rich regions, a p21-binding domain (PBD), and a kinase domain. Their length ranges from 524 amino acids to 719 amino acids. Group 1 PAKs (PAK13) have an additional auto-inhibitory domain (AID), and PAK interacting exchange (PIX) binding site whereas Group 2 PAKs (PAK4-6) have a pseudo-AID.

\subsubsection{PAK activation and regulation}

The two groups of PAKs are activated through distinctly different mechanisms. Group 1 PAKs are activated by the Rho-GTPases: Cdc42 and Rac, and may also be activated directly by growth factor receptor tyrosine kinases and G-protein-coupled receptors (Figure 1.2.1) (18, 148, 183). Regulation of group 1 PAKs is highly complex and involves molecular rearrangements, dimerisation and multiple phosphorylations $(22,120)$. Group 1 PAKs are, homeostatically, in a trans-dimer state until Cdc42 or Rac binds to PBD, leading to a series of conformational changes which destabilise the auto-inhibitory domain (AID) of PAK and 


\section{CHAPTER 1}

permit its auto-phosphorylation on certain residues in the activation loop that are important for subsequent binding to effector substrates. On the other hand, group 2 PAKs lack a defined AID but have an auto-inhibitory pseudosubstrate domain, and so are activated by different mechanisms compared to group 1 PAKs (13). Group 2 PAKs are already active in the homeostatic mono-phosphorylated state. Unlike group 1 PAKs, binding to GTPases does not increase their kinase activity, but does seem to result in their localisation to different parts of the cell (2).

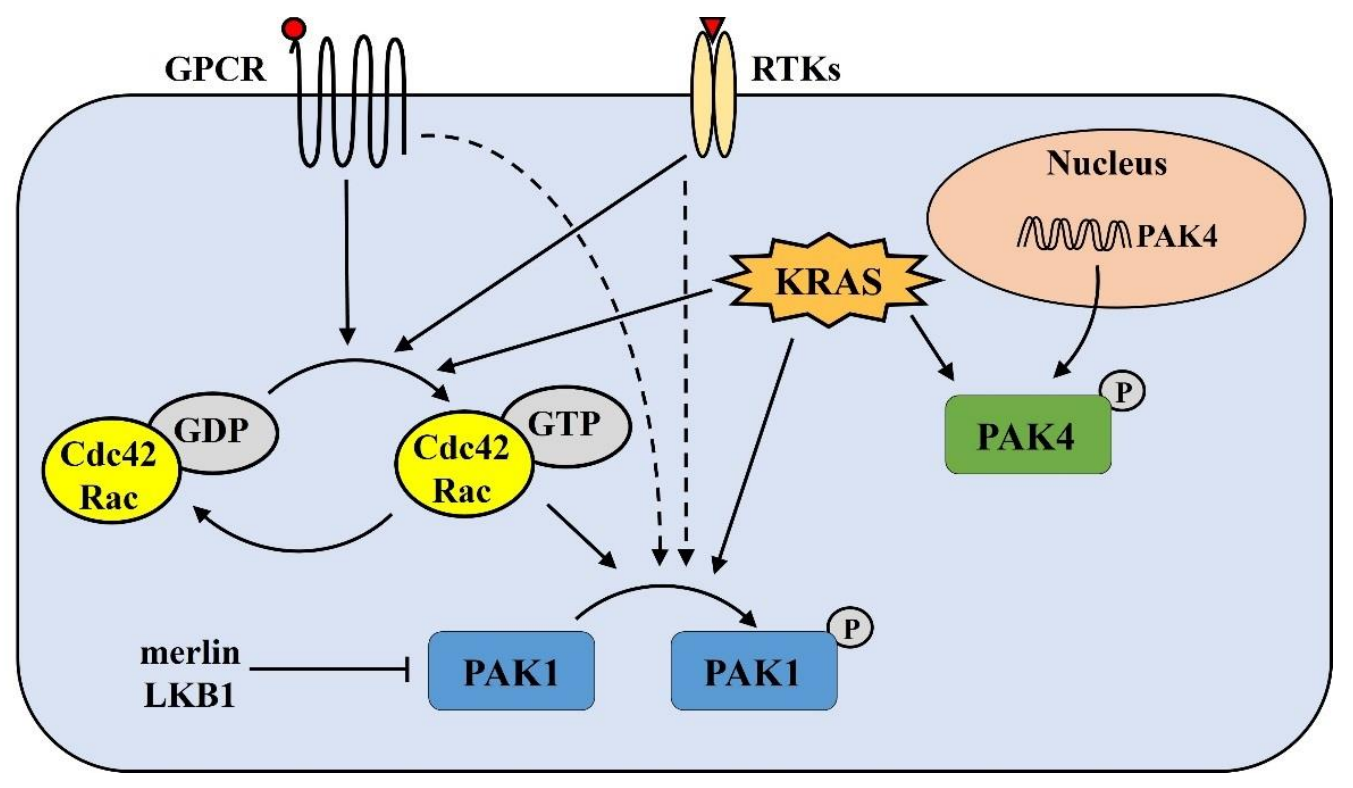

Figure 1.2.1 Regulation of PAKs differs between PAK groups in pancreatic cancer. Group 1 PAKs (PAK1) are activated when Rho-GTPases such as Cdc42 and Rac bind to the p21binding domain (PBD) transforming them to their activated phosphorylated forms, while Group 2 PAKs (PAK4) are in their activated phosphorylated forms in the resting state. $G$ protein-coupled receptors (GPCR) and receptor tyrosine kinases (RTKs) can activate signalling cascades which activate Rho-GTPases such as Cdc42 and Rac and subsequently activate PAK1, or activate PAK1 directly. However, direct activation has not been demonstrated in pancreatic cancer. The KRAS mutation, which is observed in over $95 \%$ of pancreatic tumours, can activate PAK1 directly and indirectly through activation of Cdc42 and Rac. Negative 


\section{CHAPTER 1}

regulators of PAK1 such as merlin and LKB1 (Liver Kinase B1), which normally bind to the PBD to prevent further binding, may be deregulated in pancreatic tumours, resulting in the increased activation of PAK1. PAK4 mutations is observed in around $20 \%$ of pancreatic tumours. The PAK4 gene is transcriptionally activated leading to a PAK4 protein which is already in its phosphorylated state. The KRAS mutation can increase the activity of PAK4.

Since PAKs are important in such a variety of cellular processes, the identification of endogenous negative PAK regulators has been an active field of research. The first negative PAK1 regulator to be discovered was merlin (or neurofibromin 2), which binds to the p21binding domain (PBD) of PAK1 and blocks the binding of Rac/Cdc42/Ras and thus the activation by GTPases (113). Other negative regulators include LKB1 (liver kinase B1) which also binds to the PBD of PAK1; Chp (Cdc42 homologous protein), which marks PAK1 for proteasomal degradation; CRIPak (cysteine-rich inhibitor of PAK1); PiP (PAK-interacting protein); p110C; and POPX1 and POPX2 (28, 40, 114, 203, 232, 236). Of the endogenous PAK inhibitors, nischarin, is the only one that inhibits isoforms other than PAK1, namely PAK1, PAK4 and PAK5, and regulates PAK activity by binding to its kinase domain (6). Despite the cellular and molecular importance of PAKs, our understanding of PAK regulation is still far from comprehensive. A better understanding of negative PAK regulators may give insight into how PAKs are regulated and how those mechanisms may be contributing to their upregulation during carcinogenesis.

\subsection{PAKs and Pancreatic Cancer}

Although PAKs are tightly controlled, their expression and activity can become deregulated. Upregulation or hyper-activation of PAKs has been noted in brain, breast, liver, kidney, colon, prostate, and pancreatic cancers $(44,116,156,243)$. 


\section{CHAPTER 1}

Pancreatic tumours have been found to have upregulated expression of PAK1 and PAK4 and our group and others have attempted to unravel their role and therapeutic potential. For PAK1, Chauhan and colleagues first found that MUC13, a transmembrane mucin, was overexpressed in pancreatic cancer and this correlated with increased PAK1 expression and activity (25). Following this report, Jagadeeshan and colleagues; Zhou and colleagues; and our group; individually, found that PAK1 expression in pancreatic cancer tissues was significantly higher compared to adjacent normal tissue $(102,259)$ (Chapter 4). Jagadeeshan and colleagues found that PAK1 is involved in NFKB-p65-mediated fibronectin regulation for cytoskeletal remodelling and cell transformation while Zhou and colleagues found that PAK1 mediated pancreatic cancer cell migration through hepatocyte growth factor (HGF)/MET pathway and inhibition of PAK1 with MET antagonists attenuated MET-associated resistance $(102,259)$. Lee and colleagues found that SMAD4 mutations resulted in resistance to PUMA-mediated cell death through PAK1 (118). There is also a therapeutic benefit of using PAK1 inhibitors, as our group and more recently, Jagadeeshan and colleagues, found that PAK1 is involved in gemcitabine resistance where gemcitabine docked to the active site of PAK1 and gemcitabine treatment induced PAK1 kinase activity (103) (Chapter 4). There has been growing interest in the role of PAK1 in pancreatic cancer over the past 5 years and all studies points to the clinical benefit of using PAK1-mediated therapy to improve patient outcomes.

Unlike PAK1, the PAK4 gene, on chromosome 19q13, is amplified in approximately $20 \%$ of pancreatic cancer patients $(27,111)$. PAK4 gene amplification was first identified from high resolution genome profiling of pancreatic cancer cell lines (133). Furthermore, Chen and colleagues found that PAK4 gene is not necessary mutated but instead the oncogenic form of the KRAS gene is amplified resulting in PAK4 activation and higher kinase activity (27). Kimmelman and colleagues showed that PAK4 promoted pancreatic cancer cell motility and invasion by interacting with the Rho-GTPase, Rac. Furthermore, Tyagi and colleagues and our 


\section{CHAPTER 1}

group, demonstrated PAK4 overexpression in pancreatic tumours compared to normal tissues and PAK4's role in pancreatic cancer cell proliferation and survival through AKT and ERKdependent activation of $\mathrm{NK \kappa \textrm {B }}$ signalling (210) (Chapter 3). There is also a therapeutic benefit of using PAK4 inhibitors, as PAK4 was shown to be a predictive marker of gemcitabine sensitivity, and knocking-down PAK4 in resistant cell lines resulted in increased sensitivity (158). PAK4 has also been implicated in pancreatic cancer stemness where PAK4 expression was higher in the pancreatic cancer stem cell subpopulation and was associated with stemness associated transcription factors (Oct4/Nanog/Sox2/KLF4) mediated by STAT3 signalling (211). Although PAK4 amplification was identified almost a decade ago, it has been only recently that there has been growing interest in uncovering PAK4's various roles in pancreatic cancer and thus far, studies have illustrated an advantage in using PAK4 therapy.

A study has also found a role of PAK5 in pancreatic cancer growth. Although PAK5, also known as PAK7, is not overexpressed in pancreatic cancer, to our knowledge, the study found PAK5 by using a panel of 52 molecules to inhibit specific kinases (65). They illustrated that combined inhibition of several kinases found from the panel, namely MAP3K7 and CK2 $\alpha$ with PAK5, resulted in inhibition of pancreatic cancer growth by increasing tumour apoptosis. However, no other studies have examined PAK5 inhibition in pancreatic cancer and warrants further confirmatory and exploratory studies.

There has been growing interest in the role of PAKs in pancreatic cancer over the past 5 years due to the urgent need to find effective therapeutics and understand the underlying molecular mechanisms involved in this cancer. Studies have found that PAK1, PAK4 and one study on PAK5 play a variety of roles in pancreatic carcinogenesis and their mechanisms have given insight into the complexity of the disease. However, there are still many PAK-associated mechanisms that have not been examined in pancreatic cancer and the connecting evidence to 


\section{CHAPTER 1}

key genetic and biological characteristics of pancreatic cancer will be discussed in the following section.

\subsubsection{KRAS mutation and PAKs}

Pancreatic cancer is characterised by genetic mutations, the most common of which is the KRAS oncogenic mutation (250). The KRAS gene, also known as KRAS2, is found on chromosome $12 \mathrm{p}$, and is mutated in $>95 \%$ of pancreatic cancers (23). Although mutations have been found in codons 12,13 , and 61 , the majority occur in codon 12 (G12D) in pancreatic cancer (93). The oncogenic KRAS mutations result in a constitutively active Ras protein which can in turn activate a number of signalling pathways such as MAPK, PI3K, Hedgehog, Wnt, and Notch pathways (91). In other cancers, Ras can activate Rho GTPases such as the aforementioned $\mathrm{Cdc} 42$ and Rac through direct and indirect mechanisms and consequently activate PAK1 (Figure 1.2.1) (148). The end result is increased proliferation, cell survival, and escape from apoptosis (70). Although PAK1 has not been definitely linked to the KRAS mutation, the evidence does support PAK1's activation as a downstream effector of KRAS, whilst PAK4 activity has been directly linked to the KRAS mutation in pancreatic cancer (27).

The KRAS mutation plays a pivotal role in the development and progression of pancreatic cancer. It is considered to be one of the earliest genetic mutations observed in models of the precursor lesions of pancreatic cancer. The most common precursor lesion model is pancreatic intraepithelial neoplasia (PanIN) where the KRAS mutations have been found in approximately $44 \%$ of low-grade precursors (PanIN 1) and $87 \%$ of middle-grade precursors (PanIN 2) (134, 135). Although no studies have implicated PAKs in precursor lesion models, the close relationship between activated Ras and PAK activity suggest that PAKs may be involved in the development of pancreatic cancer and warrants investigation. 


\section{CHAPTER 1}

The KRAS mutation has been linked to impaired glucose metabolism resulting in diabetes, which can assist pancreatic oncogenesis (245). Although it is unclear whether pancreatic cancer causes diabetes or diabetes causes cancer, their relationship is most likely intertwined. PAK1 has been suggested to play a role in glucose metabolism where PAK1-null mice had impaired insulin secretion and glucose tolerance (108). No long-term studies involving PAK1-null mice have been reported, though it may be an interesting study to further investigate the development of diabetes resulting to the onset of pancreatic cancer. Although no studies have directly linked KRAS mutation to PAKs resulting in diabetes and pancreatic cancer, it could be an early developmental mechanism which should be explored further.

PAKs may be involved in a number of Ras-dependent signalling pathways (Figure 1.3.1). Ras and PAKs can activate many pathways, some of which converge and reinforce the same effector function. An example is anchorage-independent growth where Ras-mediated oncogenic transformation was prevented in fibroblasts and Schwann cells when PAKs were silenced $(204,205)$. Transfection of a kinase inactive PAK prevented Ras from activating ERK and thus the MAPK pathway. PAKs can also link Ras-dependent pathways and allow crosstalk between them. One example is the MAPK and PI3K pathways which are both activated by PAK1 to increase cell growth, migration, invasion, and survival in colorectal cancer (95). Similar results have also been found in breast cancer and malignant mesothelioma $(8,149)$. Our group has confirmed these finding in pancreatic cancer for PAK1 and another group has confirmed it for PAK4 (210) (Chapter 4). Although this is only one example, it is highly likely that PAKs can mediate many pathways to increase tumour growth and metastasis. 


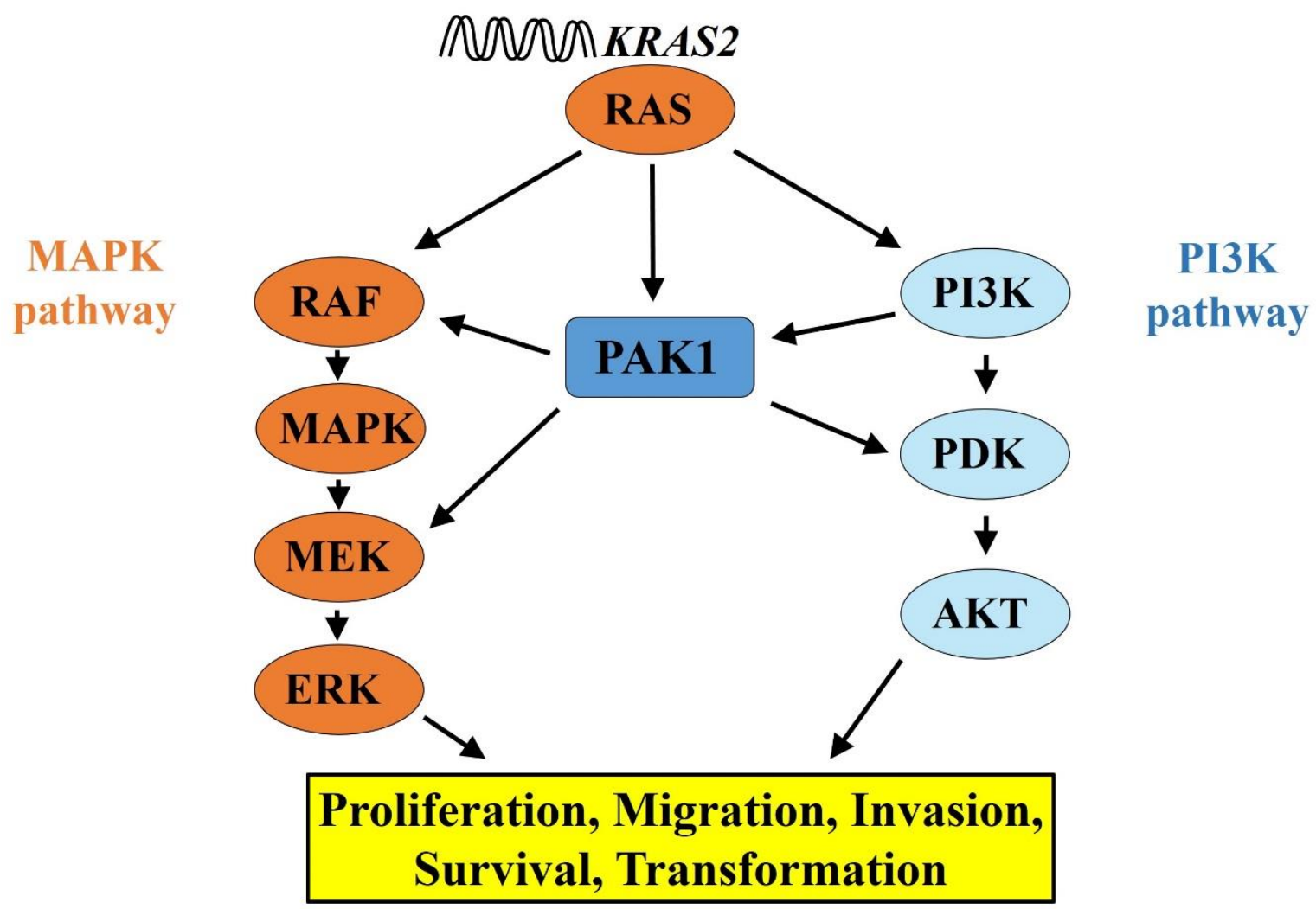

Figure 1.3.1 PAK1 involvement in Ras-dependent pathways. The KRAS mutation results in the oncogenic expression of the Ras protein. This results in the activation of a number of pathways such as the MAPK pathway through RAF, PI3K pathway through PI3K, and also activates PAK1. PAK1 can interact and mediate the cross-talk between these two pathways. Modified from (95).

\subsubsection{PAKs and Pancreatic Cancer Cell Migration and Invasion}

The involvement of PAK1 in the assembly and disassembly of focal adhesions and stress fibres contributes to pancreatic cancer cell motility and hence metastasis (156). PAKs can phosphorylate a number of cytoskeletal markers including myosin light-chain kinase (MLCK), LIM kinase (LIMK), paxillin, filamin A, stathmin, and tubulin cofactor B $(44,115,243)$. A key component of the cytoskeleton is myosin. Myosin is regulated by activated myosin light chains, which results in the assembly of myosin into bipolar filaments, forming stress fibres 


\section{CHAPTER 1}

and focal adhesions, which are found at the leading edge of the cell, and are critical for cell motility. However, disassembly is equally important. MLCK inactivates myosin light chains resulting in a decrease in activated myosin light chain and disassembly of focal adhesions and stress fibres. In the resting state, PAK1 inhibits MLCK and its ability to phosphorylate myosin light chains, resulting in reduced focal adhesions and stress fibres (189). Kaneko and colleagues showed through the use of MLCK inhibitors, that myosin is important for the invasion and metastasis of pancreatic cancer but PAK1 was not examined and PAK1-MLCK has not been studied in pancreatic cancer (106).

PAK1 and PAK4 may play a role in pancreatic cancer metastasis through the regulation of LIMK. Activated LIMK activates cofilin, an actin-binding protein, and reduces its ability to depolymerise F-actin, hence modulating actin dynamics and cell motility (44). PAK1 has been shown to activate LIMK in breast and prostate cancer and PAK4 has been shown to regulate cofilin by protein kinase D isoforms $(43,47,150)$. LIMK is involved in pancreatic cancer metastasis and also, interestingly, in cancer cell-induced angiogenesis (222). The role of PAK1 or PAK4 in relation to LIMK-cofilin has not been examined in pancreatic cancer.

The Arp2/3 protein complex is a regulator of actin filament nucleation and organisation (156). Vadlamudi and colleagues found that PAK1 interacts with p41-Arc, a regulatory component of Arp2/3 (216). They demonstrated that in breast cancer, PAK1 can activate a subunit of Arp2/3, Arpc1b, which results in increased motility. Rauhala and colleagues showed that silencing of Arp2/3 in pancreatic cancer resulted in a disruption of cell motility (181). However, as Arpc1b was expressed at low levels compared to the other subunits in pancreatic cancer, PAK1 might regulate pancreatic cancer cell migration through the interaction with p41Arc rather than Arpc1b but requires confirmatory studies. 


\section{CHAPTER 1}

Paxillin localises to focal adhesions and acts as a scaffold for the binding of other structural proteins and signalling kinases such as PAKs, which can then activate paxillin resulting in increased turnover of focal adhesions and migration (88). In pancreatic cancer, it has been found that paxillin, through c-Jun N-terminal kinase (JNK) signalling, is important for cell migration (231). PAKs can activate JNKs by inducing lamellipodia and membrane ruffling $(115,233)$. JNKs represent a second class of MAPKs, sometimes referred to as stress-activated protein kinases and is responsible for a variety of cellular process such as cell growth, differentiation, survival, apoptosis, and motility (221). However, JNK activation by PAKs is tissue specific, as PAK1 over-expression resulted in enhanced JNK activity in some cases and impaired JNK activity in others $(21,184,206)$. Also, PAKs can activate JNK indirectly, through the MAPK pathway where over-expression of MAPK has been linked with JNK activation (249). As noted above, JNK signalling is implicated in cell migration and invasion in pancreatic cancer, but a connection between PAK and JNK signalling has not been demonstrated yet $(219,231)$.

Rac and Cdc42 activate PAKs, which can then phosphorylate cytoskeletal proteins leading to changes in the actin cytoskeleton and cell mobility. Rac and Cdc42 are increased in pancreatic cancer and linked to the phosphatase and tensin homolog (PTEN) mutation in pancreatic tumours (132). PTEN, which dephosphorylates phosphatidylinositol (3,4,5)-trisphosphate $\left(\mathrm{PIP}_{3}\right)$ is a direct negative regulator of AKT, a kinase of the PI3K pathway. Deletion of PTEN in pancreatic cancer cells resulted in increased motility and higher levels of Rac and Cdc42 (192). When either Rac or Cdc42 was activated, the formation of actin rich membrane ruffles and filopodia, respectively, was induced implying possible signalling through PAKs utilising JNK, MLCK and LIMK pathways (69). 


\section{CHAPTER 1}

\subsubsection{PAKs, the EMT and Embryonic Pathways in Pancreatic Cancer}

The zinc-finger transcription factor, Snail, is a master regulator of the epithelial-mesenchymal transition (EMT) (227). Snail represses specific target genes especially those important for cell adherence and cell polarisation. PAK1 can directly phosphorylate Snail on $\operatorname{Ser}^{246}$ in breast cancer, resulting in its accumulation in the nucleus and thus, increased cell motility (242). PAK1 can also indirectly activate Snail by activating E-cadherin, occludin and aromatase promoters. Slug, a member of the Snail family of transcription factors, has been shown to play a role in metastasis in pancreatic cancer with expression of Slug, E-cadherin and MMP-9 associated with pancreatic lymph node metastases (252). Slug was found to promote cell migration and invasion by relocating MMP-9, which has been found to be a PAK1 downstream signalling target, to the leading edge. These observations highlight a possible role for PAK1/Slug and/or PAK1/Snail in pancreatic cancer metastasis.

Fibronectin is an important extracellular matrix protein and a mesenchymal marker which can aid cancer cells to become non-adherent resulting in pancreatic cancer metastasis (125). PAK1 has been found to increase fibronectin transcription through interaction with the p65 subunit of $\mathrm{NF \kappa B}$, in pancreatic cancer cell lines (102). Using promoter luciferase studies, it was found that PAK1 activated and translocated the p65 subunit to the nucleus where it activated fibronectin transcription. NFKB signalling regulates a number of effector functions and will be discussed in more detail in Chapter 1.3.4. Only a selected number of pathways have been selected and discussed but there may be many more pathways that PAK1 is implicated in pancreatic tumour migration and invasion.

Pancreatic cancer is characterised by the re-emergence of embryonic signalling pathways which are normally quiescent in the adult pancreas (187). Unlike other cancers, reactivation by a single embryonic pathway, such as Wnt, Hedgehog or Notch, is not sufficient to drive pancreatic cancer development. The Wnt signalling pathway is one of the most common 


\section{CHAPTER 1}

embryonic signalling pathways, which re-emerges in pancreatic cancer (159). In the canonical Wnt signalling pathway, Wnt binds to a Frizzled receptor which can then activate $\beta$-catenin to permit its binding to $\mathrm{T}$-cell factor 4 with subsequent activation of the transcription of target genes (187). $\beta$-catenin has been shown to be downstream of PAK1 and PAK4 in colorectal and cervical cancer, phosphorylating $\beta$-catenin directly at $\operatorname{Ser}^{675}$ resulting in the cytoplasmic accumulation and nuclear translocation of $\beta$-catenin, which in turn activates signal transduction cascades important in proliferation, morphogenesis and differentiation $(122,260)$. On the other hand, PAK1 has also been shown to antagonise the Wnt pathway through an alternative Wnt receptor, Ror, in human embryonic kidney cells, with the study concluding that PAKs could have a dual role depending on their activation mechanism, cellular type and even the subcellular compartment in which they were activated (67). Thus, the role of PAKs in the Wntsignalling pathway in pancreatic cancer has not been investigated and should be studied to determine how and the effect of PAKs on Wnt signalling.

Other embryonic signalling pathways that have re-emerged in pancreatic cancer include the Hedgehog and Notch signalling pathways (187). Interestingly, these two pathways have been linked to the KRAS mutation, further highlighting the possible role of PAKs. The KRAS mutation can suppress, through the MAPK and PI3K pathway, GLI1 protein degradation which results in activation of the Hedgehog signalling pathway $(104,152)$. Furthermore, Hedgehog signalling is activated early during pancreatic precursor lesions such as in PanINs in which KRAS mutations are said to be an early and driving mutation. Although no studies have yet linked PAKs to the Hedgehog pathway, the above observations do suggest that PAKs may have a role in the activation of the KRAS/MAPK/Hedgehog pathway.

The Notch signalling pathway involves the interaction of the membrane-bound Notch receptors, Notch1-4, and Notch ligands, such as Delta-like and Jagged, and is important for cell differentiation and homeostasis (11). Notch signalling is usually present at low levels in 


\section{CHAPTER 1}

normal adult pancreas, but in the early stages of pancreatic carcinogenesis abnormal expression of Notch ligands, a Notch1 mutant oncoprotein, and abnormal transcription of Notch signalling targets have been observed (131). The Notch signalling pathway has not been completely elucidated in pancreatic cancer and whether PAKs are involved are yet to be demonstrated (36, 77).

\subsubsection{PAKs and Pancreatic Cancer Cell Proliferation and Survival}

PAK1 can regulate a number of pathways important for tumour proliferation, survival and apoptosis. The Polo-like kinase 1 (Plk1) interacts with Aurora kinases to regulate key mitotic transitions such as centrosome maturation, spindle assembly, anaphase onset and cytokinesis. PAK1 and Plk1 co-localise on the spindle poles where they are important in establishing a functional bipolar spindle during mitosis in HeLa cells (140). Plk1 is up-regulated in pancreatic cancer and, interestingly, its expression has been linked with gemcitabine resistance (200). Under cellular stress such as gemcitabine treatment, Plk1 is depleted, preventing DNA replication, but increased Plk1 activity allows mitosis to be maintained resulting in resistance. Although the interaction between PAK1 and Plk1 has not been demonstrated in pancreatic cancer, PAK1 may mediate DNA replication through Plk1 and may also play a role in gemcitabine resistance.

Many pathways regulating motility are also involved in mitosis, especially those that require the structural reorganisation of cells such as spindle formation and cytokinesis. For example, the Arp2/3 subunit, Arpc1b, is involved in the assembly and reorganisation of actin filaments but can also interact with the Aurora kinase, Aurora-A, an important regulator of mitosis which also regulates centrosome maturation, duplication and cell cycle progression (155). Aurora-A is often upregulated in cancer and has been found to be amplified in pancreatic cancer (254). In pancreatic cancer, an Aurora-A inhibitor, CYC3, has shown promising anti-proliferative results in vitro (124). Although PAK1 can directly interact with Arpc1b, as mentioned in 


\section{CHAPTER 1}

Chapter 1.3.2, leading to changes in cell migration and cell proliferation, it is unclear if this interaction is involved in pancreatic carcinogenesis.

Cyclin D1 is an important regulator of the cell cycle and a known indicator of proliferative stage. Over-expression of PAK1 in the mammary gland has been shown to induce the transcription of cyclin D1 by the activation of NFאB (12). In pancreatic cancer, mutations in the tumour-suppressor gene p16 have been found in $95 \%$ of pancreatic tumours (137). The p16 gene encodes for a cyclin-dependent kinase inhibitor which interacts with CDK4 and subsequently cyclin D1 to inhibit the cell cycle (194). However, when p16 is lost such as in the case of pancreatic cancer due to mutation, cyclin D1 is not inhibited and cell proliferation increases. Due to the implication of PAK1 in activating NFkB pathway in pancreatic cancer, there is a high probability that PAK1 upregulates cyclin D1 via NFkB but warrants confirmatory investigation.

The NFאB signalling pathway plays an important role in maintaining homeostasis by regulating gene transcription (80). NFאB is involved in many cellular processes such as survival, tumour invasion, the stress response and drug resistance (14). NFאB is constitutively expressed in pancreatic cancer and can drive tumour progression (72). Furthermore, NFkB is activated by the KRAS mutation in pancreatic cancer (126). PAK1 and PAK4 can also activate NFkB indirectly to increase cell survival $(58,121)$. Both PAK1 and PAK4 has been shown to activate $\mathrm{NF} \kappa \mathrm{B}$ in pancreatic cancer, however the individual isoforms have resulted in different effector functions showing that more studies are needed to examine how PAK signalling influences and mediates NFkB-associated effector functions $(102,210)$.

PAKs can promote cell survival through multiple pathways. One of these pathways is through the interaction of PAKs with Bad, which in the resting state is a cell death agonist, but following binding with PAKs can protect cells from apoptosis (193). Bad belongs to the Bcl-2 family 


\section{CHAPTER 1}

which is responsible for maintaining the balance of pro-survival and pro-apoptotic signals during homeostasis. Inactivated Bad binds to and inactivates pro-survival proteins from the Bcl-2 family such as Bcl-2 or Bcl-xL, and thus promotes apoptosis. In cancer, PAK1 and PAK4 can directly phosphorylate and activate Bad, causing dissociation of Bad from Bcl-2 and/or Bcl-xL, and thus activating these pro-survival proteins resulting in tumour cell survival (66). There has been much interest in manipulating the Bcl-2 family of proteins through therapeutics to increase apoptosis and decrease gemcitabine resistance (54). The fact that gemcitabineresistant cells have an altered expression of the Bcl-2 family of proteins further highlights their importance (196). Whether this is the mechanism involved with studies finding that PAK1 and PAK4 inhibition results in gemcitabine sensitivity requires further investigation $(103,158)$.

BimL is another pro-apoptotic protein which inhibits Bcl-2 in a similar fashion to Bad. Dynein light chain 1 (DLC1) is bound to BimL and following apoptotic stimuli, BimL is released allowing it to bind and inactivate Bcl-2 (214). PAK1 can phosphorylate both DLC1 and BimL resulting in their degradation. The resultant blockade of the pro-apoptotic signal of BimL leaves Bcl-2 activated. This interplay of BimL and Bcl-2 has been studied in pancreatic cancer where it can regulate proliferation at the expense of apoptosis (3). However, the effect of PAK1 on the BimL pathway in pancreatic cancer has not yet been elucidated.

The transforming growth factor $\beta$ (TGF- $\beta$ ) pathway is involved in many cellular processes including cell cycle and migration through the transcriptional regulation of specific genes (235). TGF- $\beta$ binds to its receptor which activates receptor-regulated SMADs (rSMADs). Once activated, SMAD4 binds to these rSMADs to form complexes (rSMAD-SMAD4) which are translocated into the nucleus where they bind to promoters to regulate gene transcription. SMAD4 is important in cellular stress where it upregulates pro-apoptotic genes such as the p53 upregulated modulator of apoptosis (PUMA), through suppression of PAK1 which normally inhibits and destabilises PUMA (118). Thus, PAK1 acts as an intrinsic inhibitor of SMAD4- 


\section{CHAPTER 1}

induced apoptosis in pancreatic cancer. SMAD4 mutations, which deregulate the TGF- $\beta$ pathway, have been found in 55\% of pancreatic tumours (199). The TGF- $\beta$ pathway plays a crucial role in pancreatic cancer growth and metastasis through the transcription of many procancer genes including those that interact with PAK1.

\subsubsection{Pancreatic Cancer Stroma and PAKs}

A key characteristic of pancreatic cancer is the presence of a dense stroma that arises from the desmoplastic response. The density of this stroma results in a hypoxic environment which prevents the delivery of therapeutic agents $(129,151)$. The stroma is a dynamic milieu comprised of several different cell types including immune cells, endothelial cells, cancerassociated fibroblasts (CAFs) and pancreatic stellate cells (PSCs) (52, 164). However, targeting the stromal components of pancreatic cancer as a strategy to increase therapeutic response has thus far been unsuccessful with no clinically approved therapies (50). The stroma interaction is not fully understood and has been a growing area of research in the pancreatic cancer field.

The stroma can act as a barrier to prevent the invasion of immune cells, to allow the tumour to escape immune surveillance, and thus promote tumour survival (230). The stroma can also mediate an inflammatory response that supports tumour formation and survival. An example is CAFs' ability to mediate an inflammatory response through activation of the NFkB pathways in squamous cell carcinoma $(48,100)$. As previously noted, PAK1 and PAK4 can both activate NFkB, but PAKs have not been examined in pancreatic CAFs.

PSCs play an important role in the generation of fibrosis and release various stimuli which support carcinogenesis $(45,174)$. In the normal adult pancreas, stellate cells reside within the pancreatic parenchyma in a quiescent state. Activation of PSCs can have profound effects on tumour cells, from EMT to induction of a stem cell-like phenotype $(74,78,176)$. PAK1 has been found to be upregulated in rat hepatic stellate cells in response to injury by activation of 


\section{CHAPTER 1}

the JNK pathway, but the status of PAKs in PSCs has not been studied (except for in Chapter 5) (258).

\subsubsection{PAKs, miRNAs and HSPs in Pancreatic Cancer}

MiroRNAs (miRNAs) are a family of highly conserved 18-24 nucleotide RNA molecules that regulate the stability or translational efficiency of complementary target mRNAs (96). miRNAs have been of much interest in cancers because their genes are enriched at sites in the human genome that are often targets of amplification or chromosomal breakpoints and also due to their abnormal expression in cancer $(130,248)$. Furthermore, recent studies have found that circulating miRNAs could be used as a diagnostic and prognostic marker in pancreatic cancer and thus, have been a growing area of interest (46). In pancreatic cancer, several miRNAs, such as miRNA-21, -26a, -125b, -141a, , -145, -148a, -155, -200c, -216, -217, -221, -222, -365, $891 \mathrm{~b}$, have been found to be unregulated, and the list continues to increase $(9,73,163,191$, 202, 253, 262). In other cancers, direct links have been observed between PAKs and a number of miRNAs. The list includes miRNA-31, which is a direct target of PAK1 in colorectal cancer; miRNA-145, which impairs the $\beta$-catenin-dependent translocation of PAK4 in colorectal cancer; and miRNA let-7, which is a direct target of PAK1 in breast cancer $(92,201,240)$. Recently, miRNA let-7 was found to be implicated in pancreatic cancer such that miRNA let7 inhibited Notch and decreased pancreatic cancer progression, however PAK1's involvement was not investigated (168). The association between PAKs and miRNAs in pancreatic cancer is unclear and warrants further studies to enhance our understanding of the pancreatic cancer biology.

Heat shock proteins (HSPs) are chaperone proteins which are synthesised in response to cellular stress (109). HSPs are classified into different families based on their molecular weight and sequence homology. Only a few specific HSPs have been implicated in pancreatic cancer. HSP70 is up-regulated in pancreatic cancer and plays a role in inhibiting apoptosis and 


\section{CHAPTER 1}

increasing carcinogenesis (4). Targeting HSP70 has resulted in the development of an inhibitor, minnelide, which reduces pancreatic tumour growth and spread (31, 190). Similarly, demonstration of the involvement of HSP90 in pancreatic tumour growth and angiogenesis has resulted in the development of inhibitors, NVP-AUY922 and Y306zh $(162,239)$. Furthermore, the observations that HSP90 and HSP27 play a role in gemcitabine resistance make them ideal therapeutic targets $(64,117)$. HSP47 is over-expressed in the stroma of pancreatic cancers and may therefore be a therapeutic target for enhancing drug delivery (136). The evidence for a connection between PAKs and HSPs is limited. Rac, and presumably PAKs, can activate HSP70 through PI3K and/or JNK pathways in response to cyclic stress (20). HSP90 has been shown to involve AKT and MAPK signalling pathways and suggests possible PAK involvement (239). Furthermore activation of HSP27 by PAKs regulates actin dynamics in smooth muscle (62). The relationship between PAKs and HSPs in pancreatic cancer has not been studied and may provide insight into PAK signalling for therapeutic intervention.

\subsection{Targeting PAKs as a Therapy for Pancreatic Cancer}

The position of PAKs at a point of convergence of multiple signalling pathways makes them an appealing therapeutic target. The negative PAK regulators, as described in Chapter 1.2.1, provides some insight into how to target PAKs and can guide future therapeutic development.

The PAK endogenous inhibitors have been linked to pancreatic cancer and provides insight into their deregulation and subsequent upregulation. Merlin, a known negative PAK1 regulator, has been found to be deregulated in pancreatic cancer (Figure 1.2.1). Merlin was found to be decreased in human pancreatic tumour specimens and cell lines and regulated pancreatic cancer pathogenesis though $\beta$-catenin signalling, which is a known PAK1 target $(83,178)$. Furthermore, overexpressing merlin inhibited pancreatic cancer cell proliferation, migration and adhesion (257). Furthermore, it has been suggested that merlin may be involved as a feedback loop for NFאB and PAK1-mediated MET activation, illustrating the importance of 


\section{CHAPTER 1}

merlin in PAK1 regulation and possibly in pancreatic cancer (259). Thus, upregulating merlin to indirectly inhibit PAK1 may be an effective therapeutic strategy in pancreatic cancer.

Another PAK negative regulator that may play a role in pancreatic cancer is LKB1 (Figure 1.2.1). Interestingly, LKB1/STK11 mutations have been found in Peutz-Jeghers syndrome, a syndrome which has a $>100$-fold increase risk of pancreatic cancer $(84,146)$. Not only can the loss of LKB1 result in the increased risk of developing pancreatic cancer, but LKB1 loss has been found to synergise with oncogenic KRAS signalling to drive pancreatic cancer in vivo (161). The study also found that LKB1 is lost in $20 \%$ of human pancreatic tumours and is associated with poorer prognosis. Although the study did not link LKB1 to PAK1, the evidence that LKB1 can block PAK1 activation suggests that the loss of LKB1 may cause the upregulation of PAK1. Although two endogenous PAK1 inhibitors, merlin and LKB1, have been implicated in pancreatic carcinogenesis, suggestive of possible mechanisms of which PAK1 becomes deregulated and subsequently elevated, direct evidence is lacking and studies are needed, as insight into the events leading to the deregulation of PAK1, may guide therapeutic interventions for pancreatic cancer.

To date, a number of PAK inhibitors have been successfully discovered and created using dominant-negative mutants, RNA interference, and a number of small molecule inhibitors. However the major challenges exists in developing an effective therapeutic (33). Several small molecule inhibitors such as CEP1347, and the SRC and ETK tyrosine kinase inhibitors AG879 and FK228, respectively, have been shown to inhibit PAK activity $(82,87,165)$. The inhibitor OSU-03012, a derivative of the cyclooxygenase inhibitor, celcoxib, was developed as a PDK1 inhibitor but was later found to inhibit PAK1 activity in an ATP-competitive fashion with an $\mathrm{IC}_{50}$ of around $1 \mu \mathrm{M}$ (175). Screening of a small library of organo-ruthenium compounds led to the identification of another inhibitor FL172, which blocks the PAK1 active site with an $\mathrm{IC}_{50}$ of 100nM, but interestingly has little affinity for group 2 PAKs (138). More recently, an 


\section{CHAPTER 1}

octahedral ruthenium-based kinase inhibitor OS-2 was found with high specificity for PAK1 ( $\mathrm{IC}_{50}$ of $\left.350 \mathrm{nM}\right)$ over other kinases (55). However, the affinity of OS-2 for group 2 PAKs was not reported. The discovery of FL172 and OS-2 illustrates the usefulness of using octahedral metal complexes as scaffolds in developing highly selective and potent kinase inhibitors.

The reported ATP-competitive inhibitor FRAX597 selectively inhibits group 1 PAKs (123). This oral inhibitor, which was found using a structure-activity relationship approach through high-throughput screening of a library of small molecules, inhibited proliferation in neurofibromin 2-associated schwannomas. FRAX597 is specific to group 1 PAKs, with $\mathrm{IC}_{50}$ values for PAK1, PAK2, and PAK3 of $8 \mathrm{nM}, 13 \mathrm{nM}$, and $19 \mathrm{nM}$, respectively, and the $\mathrm{IC}_{50}$ value for PAK4 is greater than 10mM. Another ATP-competitive inhibitor is LCH-7749944, which binds to PAK4 with an $\mathrm{IC}_{50}$ of $15 \mu \mathrm{M}$ (251). This inhibitor was developed using structureinformed design and has been found to block the effects of PAK4 downstream targets such as LIMK1, cofilin, MAPK and EGFR. PF-3758309 is a pyrrolopyrazole PAK4 inhibitor designed by Pfizer (71). Although it has the highest affinity for PAK4 ( $\left.\mathrm{IC}_{50} 2.7 \mathrm{nM}\right)$, it has been shown to inhibit both group 1 and group 2 PAKs, perhaps due to the similarities in active sites. Unfortunately, a phase 1 trial of PF-3758309 was stopped due to undesirable pharmacokinetic characteristics (1). Thus, ATP-competitive inhibitors are useful and potent, but often do not discriminate between specific PAK isoforms due to the similarities in the active site.

An alternative to ATP-competitive kinase inhibitors is the class of allosteric inhibitors, which bind to sites other than the ATP-binding pocket. A major advantage of allosteric inhibitors is the potential to achieve greater specificity compared to the ATP-competitive inhibitors. By developing a screen for allosteric inhibitors targeting PAK1 activation, IPA-3 (inhibitor of p21activated kinase-3), was identified (38). IPA-3 is specific to group 1 PAKs, with an $\mathrm{IC}_{50}$ of $2.5 \mu \mathrm{M}$ for PAK1, and limited inhibitory capacity towards group 2 PAKs. IPA-3 binds covalently to the regulatory domain of PAK1, and blocks the binding of Cdc42 (220). However, 


\section{CHAPTER 1}

IPA-3 is unlikely to be a useful tool for inhibiting PAK1 activity in biological systems as, although the exact nature of the bonds is unknown, the reaction is reversible in target cells and therefore IPA-3 has not been pursued further (255). Nevertheless, it does highlight the advantage of targeting activating mechanisms rather than kinase sites.

Alternative approaches include the use of dominant-negative forms or fragments of PAKs to prevent activity. These molecules take the place of PAKs in complexes with other proteins and thereby prevent the activation of downstream signalling pathways (112). Potential problems with this technique are that the molecules may affect both kinase-dependent and -independent functions, and also may not be isoform specific since PAKs share many common interactions. Another approach is the use of RNAi (RNA interference) to inhibit PAKs. Group 1 PAKs have been depleted using shRNA to inhibit proliferation and cancer progression in neurofibromatosis type 2 (244). This method provides better specificity but its clinical utility is questionable and would require a vehicle to traffic and enter pancreatic tumours.

In summary a handful of PAK inhibitors have been developed, but have not been successful in the clinical setting. Although many approaches have been outlined to discovering PAK inhibitors, none have yet resulted in a safe, specific and effective inhibitor. Furthermore, the complex regulatory mechanisms and multiple effector functions of PAKs make the development of ATP-competitive inhibitors difficult, and may favour the development of allosteric PAK inhibitors.

\subsection{Summary and Research Aims}

Pancreatic cancer is a human malignancy with a poor prognosis that has not improved significantly over the past few decades. Improvement of our current management will require a comprehensive understanding of the underlying biological mechanisms. PAKs, with their ability to activate many cellular processes, have thus far been shown to have a significant role 


\section{CHAPTER 1}

in a number of pancreatic cancer signalling pathways, however, their comprehensive role is still needed and the indirect and connecting evidence have been discussed. Studies have found that upregulation and/or hyper-activation of PAK1 and PAK4 are involved in pancreatic cancer growth and metastasis, and chemosensitivity. In particular, the effect of PAKs on multiple pathways makes PAK-directed therapy an attractive option, after further refinement, for the treatment of pancreatic cancer.

Thus, more research is required to unravel its role and evaluate the clinical effectiveness of PAK inhibitors on pancreatic cancer. PAK's involvement in chemosensitivity provides evidence for combinational therapy with the addition of PAK inhibitors to standard treatments to increase sensitivity and reduce resistance. The effect of PAK inhibitors on the pancreatic cancer stroma is also needed and may assist in unravelling the complexity of the tumour-stroma interaction and lead to more effective and targeted therapies. The overall objective of this project is to determine the role of PAK1 in pancreatic cancer and evaluate the therapeutic potential of inhibiting PAK1.

The specific hypotheses are as follows:

1. PAK1 and PAK4, together, play a role in pancreatic cancer growth and migration/invasion and dual inhibition increases murine survival (Chapter 3)

2. PAK1 plays a role in pancreatic cancer growth, migration/invasion, and chemosensitivity and PAK1 inhibition with gemcitabine increases murine survival (Chapter 4).

3. PAK1 plays a role in PSCs proliferation, apoptosis and stellate cell activation and is involved in the interaction between PSCs and pancreatic cancer cells (Chapter 5). 


\section{CHAPTER 1}

The specific aims are as follows:

1. To evaluate the effects of an indirect PAK inhibitor, glaucarubinone, alone and in combination with standard chemotherapy agent, gemcitabine, on pancreatic cancer growth, migration/invasion, expression and activation of PAK1 and PAK4 in vitro and in vivo, and murine survival in vivo (Chapter 3 )

2. To examine the effects of reducing PAK1 activity, with the group 1 PAK inhibitor, FRAX597, or PAK1 expression by shRNA knockdown, alone and in combination with gemcitabine, on pancreatic cancer growth and migration/invasion in vitro, and in vivo, and murine survival in vivo (Chapter 4$)$.

3. To evaluate the effect of reducing PAK1 activity with a group 1 PAK inhibitor, FRAX597, on PSC activation, activity, proliferation and apoptosis, in vitro and evaluate the role of PAK1 in the interaction between PSCs and pancreatic cancer cells in vitro and in vivo (Chapter 5). 
CHAPTER 2

Chapter 2: General Material and Methods 


\section{CHAPTER 2}

\subsection{Introduction}

To investigate the role of PAKs in pancreatic cancer, pancreatic cancer cell lines and mouse models were utilised. Human and mouse pancreatic cancer cell lines were used for in vitro experiments. Studies presented in Chapter 3, 4, and 5 utilised PAK inhibitors to demonstrate the effect of inhibiting PAK1 on these cell lines and their effect on pancreatic cancer cells using assays to measure proliferation, survival, toxicity, migration/invasion and signalling protein changes. In addition, Chapter 4 utilised PAK1 stable knockdown cell lines to further evaluate the effect of targeting PAK1. Chapter 3, 4 and 5 implemented identical orthotopic preclinical mouse models where murine pancreatic cancer cell lines were implanted into the pancreas of immunocompetent (C57B1/6) mice. Methods specific to individual studies can be found within each chapter.

All mouse experiments were performed in strict accordance with the guidelines of the National Health \& Medical Research Council of Australia Code of Practice for the Care and use of Animals for Experimental Purposes in Australia. The protocol was approved by the Austin Health Animal Research Ethics Committee (A2013/04898). Mice were acclimatised prior to all experimental procedures. Continuous monitoring was included throughout the length of the study, as per animal ethics guidelines. Mice were scored based on weight, coat and body condition and physical response to handling during routine husbandry (Appendix i).

Human tissue experiments involved the collection, processing and analysis of human samples. The protocol was approved by the Austin Health Human Research Ethics Committee (H2013/04953). Fresh tissue was obtained from residual specimens of patients undergoing surgical resection. This included patients undergoing surgical resection for primary pancreatic adenocarcinoma or pancreatic liver metastases, and partial or total pancreatectomy for other reasons such as chronic pancreatitis. The tissue, tumour and normal, were cut into blocks and fixed for hematoxylin and eosin (H\&E) and immunohistochemical (IHC) staining, snap frozen 


\section{CHAPTER 2}

for Western blot analysis, or cryopreserved in cell freezing media for later experimentation such as stellate cell isolation in Chapter 5. Tissues were coded to maintain patient confidentiality and privacy as per human ethics guidelines.

\subsection{Cells and Reagents}

The human PANC-1, MiaPaCa-2, AsPC-1 and BxPC-3 (American Type Culture Collection, Manassas, USA) and the murine Pan02 (Division of Cancer Treatment and Diagnosis Tumour Repository, NCI, Frederick, USA) and LM-P (obtained from Andrew Lowy (Moores Cancer Center, University of California, San Diego, USA) (209)) pancreatic cancer cell lines were cultured in Dulbecco's Modified Eagle's Medium (DMEM) supplemented with $10 \%$ heatinactivated FBS (foetal bovine serum; Hyclone Laboratories Inc., Scoresby, Australia). Normal immortalised human pancreatic duct epithelial (HPDE) cells (originally derived from M.S Tsao (Ontario Cancer Institute, Ontario, Canada) (179)) were cultured in Keratinocyte serum-free medium supplemented with bovine pituitary extract (BPE) and epidermal growth factor (EGF). All cells were cultured in a $37^{\circ} \mathrm{C}$ incubator with $5 \% \mathrm{CO}_{2}$. Cells were tested regularly for mycoplasma contamination and were not passaged more than 30 times or for more than 6 months after revival.

Human pancreatic stellate cells (hPSC) were prepared by the outgrowth method (10). Human tumour samples were cut into fragments and placed into a culture dish, cultured in $20 \%$ FBS in DMEM with penicillin/streptomycin. After 5-10 days, tumour fragments were transferred to a fresh culture dish where outgrowth of PSCs occurred from the fragments. Fragments were then removed after 2-4 weeks and PSCs allowed to grow to confluence. Medium was changed every 3 days. The human pancreatic stellate cell line, hPSC1 (ScienCell Research Laboratories, Carlsbad, CA, USA), which was isolated and established from human pancreas, was cultured in DMEM supplemented with $10 \%$ FBS. hPSC1 cells were found to be similar to the other 


\section{CHAPTER 2}

isolated hPSCs in morphology and staining markers. Cells were cultured in a $37^{\circ} \mathrm{C}$ incubator with $5 \% \mathrm{CO}_{2}$ and not passaged more than 6 times.

Normal mouse PSCs (nmPSC) were isolated from 5 pooled pancreata using the density gradient centrifugation method, based on the rat PSC isolation method (7). The pooled pancreases were isolated and placed in ice-cold saline. Adipose, connective tissue and any large blood vessels were removed and the pancreases minced and digested with enzyme solution which consisted of protease, collagenase P and DNAse (Sigma Aldrich, Castle Hill, Australia) on a shaker at $37^{\circ} \mathrm{C}$. Digested tissue was then spun down at $1700 \mathrm{rpm}$ for 10 minutes at $4^{\circ} \mathrm{C}$ and resuspended in nycodenz (Histodenz, Sigma Aldrich) with a final concentration of $11.4 \%$ and spun down at 2900rpm for 20 minutes at $4^{\circ} \mathrm{C}$. The band just above the interface was harvested and contained PSCs. nmPSC were cultured in 20\% FBS in Iscove's modified Dulbecco's medium (IMEM) with penicillin/streptomycin. Cells used for experiments were between passage 3 and 6 .

Gemcitabine hydrochloride was purchased from Sigma-Aldrich (Castle Hill, Australia), reconstituted in saline and stored at $4^{\circ} \mathrm{C}$, was used for all in vitro experiments. Gemzar ${ }^{\circledR}($ Eli Lilly) purchased from Austin Health Pharmacy (Heidelberg, Australia), the clinical formulation of gemcitabine, was used for all in vivo experiments. This formulation included hydrochloric acid, sodium hydroxide, mannitol and sodium acetate, was diluted with saline and was stored at $4^{\circ} \mathrm{C}$.

Glaucarubinone, given by Dr. John Beutler (NIH, Fredrick, USA), was dissolved in saline and stored at $4^{\circ} \mathrm{C}$.

FRAX597 was purchased from SYNthesis (Parkville, Australia), dissolved in saline and stored at $4^{\circ} \mathrm{C}$. 


\section{CHAPTER 2}

\section{3 shRNA Transfection}

To obtain PAK1 knockdown (KD) clones, cells were transfected with SureSilencing shRNA plasmids for human PAK1 (SABioscience, Doncaster, Australia), or with a scrambled sequence as a negative control (NC), using Lipofectamine2000 (Invitrogen, Mulgrave, Australia). $4 \mu \mathrm{g}$ of plasmid DNA, or NC, was mixed with DMEM and added to the Lipofectamine2000/DMEM mixture and incubated for 20 minutes at room temperature. The DNA/Lipofectamine2000 mixture was then added to the cells for 6 hours and geneticin (G418) was added to the cells the following day after transfection. When confluent, cells were passaged at low concentration for colony selection. Stable clones were then selected by scraping colonies with a pipette tip and transferring them to a 24 -well plate where they were allowed to grow. Once confluent, they were tested for PAK1 protein expression by Western blot where 2 NC clones and 2 positive PAK1 KD clones were chosen from each cell line.

\subsection{Cell Proliferation, Survival and Toxicity Assays}

Cell proliferation, survival and toxicity was measured using ${ }^{3} \mathrm{H}$-thymidine assays. For cell proliferation, cells were seeded at a density of $5 \times 10^{3}$ cells/well in DMEM containing $10 \%$ FBS and allowed to adhere for 24 hours. A haemocytometer was used for the counting of cells. To determine the level of DNA synthesis, $1 \mu \mathrm{Ci} /$ well $\left[\right.$ methyl- $\left.{ }^{3} \mathrm{H}\right]$-thymidine (Perkin Elmer; Boston, USA) was added for 24 hours with treatment. Cells were then harvested using a NUNC cell harvester and the radioactivity was measured in duplicate by liquid scintillation spectrometry with a $\beta$-counter (Packard; Connecticut, USA). For combinational effect on proliferation, cells were pre-treated with a PAK inhibitor, 4 hours after seeding. 20 hours afterwards, media was removed and increasing concentrations of gemcitabine with $1 \mu \mathrm{Ci} /$ well [methyl- $\left.{ }^{3} \mathrm{H}\right]$-thymidine was added. 24 hours later, cells were harvested and detected as per above. For cell proliferation over a number of time periods, cell growth curves were fitted 


\section{CHAPTER 2}

based on a log-scale using MATLAB (MathWorks, Natick, MA) and expressed as a growth rate $(\% / \mathrm{hr})$.

For cell survival and cell toxicity, cells were seeded as above however $1 \mu \mathrm{Ci} /$ well $\left[\right.$ methyl- $\left.{ }^{3} \mathrm{H}\right]-$ thymidine was also added and cultured for 24 hours. Free, unincorporated, ${ }^{3} \mathrm{H}$-thymidine was then removed and cells were washed with PBS. Cells were then cultured with $10 \%$ serum - to determine toxicity - or without $10 \%$ serum - to determine survival - for a further 24 hours. The cells were harvested and detected as described above. Measurements were calculated as a ratio to the $10 \%$ serum control.

Cell proliferation was also measured using the MTT assay. This was done with 5-FU treatment since 5-FU inhibited the synthesis of thymidine nucleotides and thus, ${ }^{3} \mathrm{H}$-thymidine assay could not be used. Like with the ${ }^{3} \mathrm{H}$-thymidine assay, cells were seeded at a density of $5 \times 10^{3}$ cells/well in DMEM containing 10\% FBS and allowed to adhere for 24 hours. A haemocytometer was used for the counting of cells. Increasing concentrations of 5-FU was added. 24 hours later, $10 \mu \mathrm{l}$ of $5 \mathrm{mg} / \mathrm{ml}$ MTT (3-(4,5-dimethylthiazol-2-yl)-2,5diphenyltetrazolium bromide) was added to each well and incubated for 3 hours. Cellular metabolic enzymes reduce MTT to its insoluble formazan crystals which has a purple colour. Media was removed and $100 \mu \mathrm{l}$ acidified isopropanol was added and placed on a moving platform for 5 mins, protected from light. This dissolves the purple crystals and resulting purple solution is spectrophotometrically measured by reading at an absorbance of 570nm with a reference filter of $620 \mathrm{~nm}$ on a 550 microplate reader (Bio-Rad, Australia). For combinational effect on proliferation, cells were pre-treated with FRAX597, 4 hours after seeding. 20 hours afterwards, media was removed and increasing concentrations of 5-FU was added. 24 hours later, MTT was added and read as per above. 


\section{CHAPTER 2}

\subsection{Combination Index}

The combined effects of a PAK inhibitor and gemcitabine were evaluated using a combination index (CI). The collated proliferation values from Chapter 2.4 for PAK inhibitor alone, chemotherapy alone, and the combination were used. The CalcuSyn program (T.C. Chou and M.P. Hayball; Biosoft; Cambridge, UK) were used to calculate and analyse the CI using the Chou-Talalay method (the isobologram equation was used mutually non-exclusive $(\alpha=1)$ ) (29). The CI for each fraction-affected value $\left(\mathrm{F}_{\mathrm{a}}\right)$ represents the percentage of proliferation inhibition. The combination index is interpreted as: $<1.0$, synergistic; 1.0 , additive and $>1.0$, antagonistic.

\subsection{Sphere Formation Assay}

Sphere formation assay determines clonogenic growth potential for the subset of cancer stem cells. Cells were seeded at a density of $5,10,20 \times 10^{3}$ cells/well in serum-free DMEM containing $10 \mathrm{ng} / \mathrm{ml}$ bFGF (Wako Pure Chemical Industries, Japan), $10 \mu \mathrm{g} / \mathrm{ml}$ human insulin (Cell Science and Technology Institute, Japan), $100 \mu \mathrm{g} / \mathrm{ml}$ human transferrin (Roche Life Science, Castle Hill, Australia) and $100 \mu \mathrm{g} / \mathrm{ml}$ BSA (Sigma-Aldrich) in 24-well Ultra Low Attachment plates for 10 days. Wells were then counted for sphere formation, in a blinded manner, using the microscope at 40x magnification. Wells were also imaged using an EVOS cell imaging system (Thermo Fisher Scientific) and representative images chosen.

\subsection{Cell Apoptosis Assay}

Cell apoptosis was assessed using the annexin V-FITC apoptosis detection kit (BioVision, Milpilas, CA, USA). Annexin V binds to cell surface phosphatidylserine which translocates from the inner face of the plasma membrane to the cell surface when apoptosis is initiated. PI binds to necrotic cells, differentiating cells undergoing apoptosis and necrosis. Cells were seeded in 6-well plates for 24 hours. Cells were then collected, spun down and resuspended in 


\section{CHAPTER 2}

$500 \mu \mathrm{l}$ of $1 \mathrm{X}$ binding buffer. $5 \mu \mathrm{l}$ of annexin V-FITC and $5 \mu \mathrm{l}$ of propidium iodide (PI) was added and incubated at room temperature for 5 minutes in the dark. Cells were then analysed by flow cytometry where PI vs FITC was plotted following gating based on cell size.

\subsection{Migration/Invasion Assay}

Cell migration/invasion was measured using the Transwell Boyden Chamber assay. Membranes $(8-\mu \mathrm{m}$ pore size, Falcon®, Corning, Tewksbury, USA) were coated with $3 \mu \mathrm{g}$ of human fibronectin on the lower surfaces and placed into a 24-well plate containing serum-free DMEM with $0.1 \%$ bovine serum albumin (BSA). $5 \times 10^{4}$ cells were added to the upper chambers and incubated for 24 hours. Non-penetrated cells were removed by wiping with a cotton swab from the upper surface. The membranes were fixed, stained with Quick-Dip (Fronine; Sydney, Australia), cut, mounted in an organic mountant, DPex (VWR International Ltd; QLD, Australia), cover-slipped and allowed to dry overnight. The cells that had migrated to the lower surface of the membranes were counted on 48 fields, at 400 times the magnification using a NIKON Coolscope (Coherent Scientific; Adelaide, Australia).

\subsection{Hypoxia Treatment of Cells for Western Blot}

Protein expression was determined in cells cultured under normoxic or hypoxic $\left(1 \% \mathrm{O}_{2}\right)$ conditions. For hypoxia incubated cells, cells were seeded in $24-w e l l$ plates and placed into a sealed humidified chamber containing $94 \% \mathrm{~N}_{2}, 5 \% \mathrm{CO}_{2}$ and $1 \% \mathrm{O}_{2}$ for 24 hours before protein extraction. Oxygen concentrations were automatically adjusted by an electronic oxygen controller (ProOx Model 110; Biospherix, Parish, USA). For normoxia, cells were similarly seeded and proteins extracted concurrently with the hypoxia incubated cells but were incubated in normal incubator conditions. Protein extraction and Western blot followed as described below. 


\section{CHAPTER 2}

\subsection{Western Blot}

Proteins were extracted from cells seeded in 24 -well plates for at least 24 hours with $\sim 80 \%$ confluency. For treated cells, single agent treatment was added 24 hours prior to extraction or in the case of double agent treatment, PAK inhibitor was incubated for 6 hours, removed and incubated with gemcitabine for 20 hours before extraction. For protein extraction, media was removed, cells washed and 2x SDS (sodium dodecyl sulphate) sample buffer was added. Samples were collected, heated at $95^{\circ} \mathrm{C}$ for 5 minutes and stored at $-20^{\circ} \mathrm{C}$ until needed.

For Western blot, samples were electrophoresed on 10\% SDS-polyacrylamide gel by standard electrophoresis methods at 150V. Resolved protein was transferred onto Hybond-C Extra nitrocellulose membrane (GE Healthcare, Rydalmere, Australia) at $100 \mathrm{~V}$ for 1 hour. Membranes were blocked for 1 hour in 5\% skim milk in TBST (tris-base saline plus $0.1 \%$ Tween 20) then washed with TBST and incubated overnight with primary antibody (see Table 2.10 for primary antibodies and dilutions) at $4{ }^{\circ} \mathrm{C}$ on a moving platform. Primary antibody was removed, membranes were washed with TBST and incubated with the appropriate HRP(horseradish peroxidise) conjugated secondary goat antibody (1:10000; Biorad, Gladesville, Australia) for 1 hour at room temperature on a moving platform. Membranes were thoroughly washed with TBST and protein bands were visualised in a LAS 3000 Image Reader (Fujifilm, Brookvale, Australia), with the chemiluminescence reagent, ECL Advance Western Blotting Detection Kit (GE Healthcare, Buckinghamshire, UK). The band densities were analysed using Multigauge computer software (Berthold, Bundoora, Australia) and calculated to the corresponding GAPDH band. 
Table 2.10: Primary Antibodies used for Western Blotting

Target Protein Antibody Supplier (catalogue number) Dilution

\begin{tabular}{|c|c|c|c|}
\hline AKT & Monoclonal Rabbit & Cell Signaling Tech. (4685) & $1: 2500$ \\
\hline$\alpha \mathrm{SMA}$ & Monoclonal Rabbit & Abcam (ab32575) & $1: 2500$ \\
\hline$\beta$-catenin & Polyclonal Rabbit & Cell Signaling Tech. (9587S) & $1: 5000$ \\
\hline Collagen I & Polyclonal Rabbit & Abcam (ab34710) & $1: 5000$ \\
\hline cyclinD1 & Monoclonal Rabbit & Cell Signaling Tech. (2978S) & $1: 1000$ \\
\hline desmin & Polyclonal Rabbit & Santa Cruz (sc-14026) & $1: 1000$ \\
\hline ERK1/2 & Polyclonal Rabbit & Cell Signaling Tech. (9102L) & $1: 2500$ \\
\hline GAPDH & Monoclonal Rabbit & Cell Signaling Tech. (2118L) & $1: 5000$ \\
\hline HIF $1 \alpha$ & Monoclonal Mouse & $\mathrm{BD}(610958)$ & $1: 1000$ \\
\hline MMP9 & Polyclonal Rabbit & Ana-Spec (29579) & $1: 250$ \\
\hline PAK1 & Polyclonal Rabbit & Cell Signaling Tech. (2602S) & $1: 2500$ \\
\hline PAK2 & Monoclonal Rabbit & Cell Signaling Tech. (2615S) & $1: 2500$ \\
\hline PAK4 & Polyclonal Rabbit & Cell Signaling Tech. (3242S) & $1: 5000$ \\
\hline pAKT & Polyclonal Rabbit & Cell Signaling Tech. (9271S) & $1: 2500$ \\
\hline pERK & Polyclonal Rabbit & Cell Signaling Tech. (9101S) & $1: 2500$ \\
\hline $\mathrm{pPAK} 1 / 2$ & Polyclonal Rabbit & Cell Signaling Tech. (2601S) & $1: 1000$ \\
\hline
\end{tabular}


CHAPTER 2

$\begin{array}{llll}\text { pPAK4/5/6 } & \text { Polyclonal Rabbit } & \text { Cell Signaling Tech. (3241S) } & 1: 1000 \\ \text { VEGF } & \text { Polyclonal Rabbit } & \text { Santa Cruz (sc-507) } & 1: 1000 \\ \text { Vimentin } & \text { Polyclonal Rabbit } & \text { Santa Cruz (sc-5565) } & 1: 250\end{array}$

\subsection{Experimental animals}

SCID mice (6 weeks old) were purchased from Animal Resource Centre (Perth, Australia) for the xenograft flank model (Chapter 2.12). C57Bl/6 mice (8-10 weeks old) were either purchased from Walter and Eliza Hall (Parkville, Australia) or inbred at the Austin Bioresource Facility (Heidelberg, Australia) for the orthotopic murine model (Chapter 2.13). Animals were housed in groups of up to five in the standard cages in a room maintained at constant temperature and humidity with 12-hour light and dark cycles, and fed with regular autoclaved chow diet with water ad libitum. None of the mice exhibited any lesions and were pathogen-free before commencement of any experimentation.

\subsection{Subcutaneous Flank Murine Model}

The flanks of mice were shaved and tumour cells were implanted subcutaneously into each flank using a 26-gauge needle. Tumour dimensions were measured every other day using micro-callipers, and volumes (V) calculated using the formula:

$$
V=W^{2} \times \frac{L}{2}
$$

where $W$ and $L$ are the shortest and longest tumour diameters, respectively.

Mice were sacrificed at the endpoint, tumours extracted, weighed, and harvested for histological analysis. 


\section{CHAPTER 2}

\subsection{Orthotopic Pancreatic Cancer Murine Model}

Established murine models of pancreatic cancer involving the orthotopic implantation of murine cancer cells into the pancreatic head or tail was utilised for preclinical evaluation (166). As previously characterised, pancreatic head murine model was utilised for survival studies and pancreatic tail murine model was utilised for tumour growth studies.

Mice were anesthetised with an intraperitoneal (i.p.) injection of ketamine (100mg/kg; Pfizer; West Ryde, Australia) and xylazine (10mg/kg; Troy Laboratories Pty Ltd; Glendenning, Australia) solution at a dose of $100 \mu \mathrm{l}$ per $1 \mathrm{~g}$ of body weight. Carprofen $\left(50 \mathrm{mg} / \mathrm{ml}\right.$; Rimadyl ${ }^{\circledR}$; $5 \mathrm{mg} / \mathrm{kg}$; Pfizer), an analgesic for pain alleviation, was given subcutaneously at the back of the neck. Before, during and after surgery, Polyvinc eye gel (Alcon Laboratories (Australia) Pty Ltd, Frenchs Forest, Australia) was administered for eye protection. Body temperature was maintained during surgery by performing all procedures on heat pads and following surgery by recovering animals in cages warmed by heat pads.

The abdomen and below the left costal margin was shaved and a small left transverse, abdominal flank incision was made to expose the pancreas. For the pancreatic head model, the head of the pancreas was identified by locating the duodenum, grasped and rotated to expose the posterior aspect of the pancreatic head. For the pancreatic tail model, the tail of the pancreas was identified by finding the left end of the pancreas, in contact with the spleen. Murine cells were directly injected into the pancreas using a calibrated syringe (Hamilton Syringe; SigmaAldrich) and a 27-gauge needle. Tumour cell leakage into the abdominal cavity during injection was prevented by using a cotton swab held over the site of injection following injection and only those that had no tumour cell leakage was used for experimentation. For rehydration following surgery, animals were given $1 \mathrm{ml}$ of saline i.p.. The abdominal muscle and skin layers were sutured separately using 4.0 silk sutures (Ethicon Inc., Johnson \& Johnson, New Brunswick, USA). The wound was sterilized using $0.05 \%$ aqueous chlorhexidine solution. 


\section{CHAPTER 2}

Post-surgery, mice were allowed to recover in cages warmed on heat pads with access to raspberry jelly, irradiated rodent pellets and water. The surgical procedure was well tolerated with minimal $(<5 \%)$ procedure or anaesthesia-related deaths. Mice were monitored by daily weighing and health scored (appendix i) for the first week following surgery then 3 times a week thereafter.

For the pancreatic head model, mice were monitored for signs of deteriorating health and were monitored closely, given a 'wet' diet, and/or sacrificed if the health score was reached. Day 1 was considered the day of surgery/pancreatic induction. At the endpoint, all mice were sacrificed and the mice were visually inspected for tumours, obvious metastasis, and its organs harvested for histological analysis. Survival was presented using the Kaplan-Meier method, with collation when necessary (SPSS; IBM, New York, USA).

For the pancreatic tail model, mice were monitored for up to 40-50 days (depending on the study). At the endpoint, mice were sacrificed and were visually inspected for obvious metastasis, the primary tumour was located and a single investigator measured the dimensions of the tumour using micro-calipers, in a double-blinded manner. Organs were then harvested for histological analysis. Tumour volume (V) was calculated using the formula for ellipsoid tumours:

$$
V=L x W x H x \frac{\pi}{6}
$$

where $\mathrm{L}$ was the longest distance from right to left; $\mathrm{W}$, the largest dorsal/ventral diameter; and $\mathrm{H}$, the largest rostral/caudal diameter.

\subsection{Tissue Processing}

Harvested organs were fixed in 10\% phosphate buffered formalin (Sigma-Aldrich) at room temperature for 24 hours and then transferred to $70 \%$ ethanol. Organs were placed between 


\section{CHAPTER 2}

70\% ethanol soaked biopsy pads (Grale Scientific, Ringwood, Australia) in tissue processing cassettes (Simport, Beloeil, Canada). The cassettes were then immersed in $70 \%$ ethanol and processed by the Biomedical Sciences Histology Facility (Level 2, School of Biomedical Sciences, The University of Melbourne, Parkville, Australia) and embedded in paraffin wax blocks using an overnight processing schedule. This processing schedule allowed tissues to undergo dehydration through an increasing series of graded ethanol solutions. Tissues were then immersed in three changes of xylene. Samples were then vacuum-embedded in paraffin wax to produce formalin fixed paraffin embedded (FFPE) tissue blocks. The FFPE blocks were then cut into $4 \mu \mathrm{m}$ sections using a microtome (Leica RM2245, Wetzlar, Germany) and mounted onto either a glass slide (Menzel Glaser®, Thermo Fisher Scientific, Scoresby, Australia) for H\&E Staining, or a Superfrost plus slide (Menzel-Glaserslides, Braunschweig, Germany) for IHC. Sections were dried in a $37^{\circ} \mathrm{C}$ oven overnight prior to staining.

Tumour protein expression was analysed from snap-frozen samples. Similar weights of tumour tissue were taken and the proteins extracted by liquid nitrogen grinding and homogenising. Samples were placed in foil and dipped in liquid nitrogen and a mortar used to grind and pulverise the samples into small pieces and then transferred to a tube with $1 \%$ Triton $\mathrm{X} 100$ lysis buffer (50 mM HEPES, $150 \mathrm{mM} \mathrm{NaCl}, 10 \mathrm{mM}$ EDTA, $10 \mathrm{mM} \mathrm{Na}_{4} \mathrm{P}_{2} \mathrm{O}_{7}, 100 \mathrm{mM} \mathrm{NaF}$, $5 \mu \mathrm{g} / \mathrm{ml}$ aprotinin, $5 \mu \mathrm{g} / \mathrm{ml}$ leupeptin and $1 \mathrm{mM}$ PMSF). Tissue samples were homogenised (ultra-turrax T25, Janke and Kunkel, Ika ${ }^{\circledR}$ Labortechnik, Staufen, Germany) and then centrifuged for 10 minutes at $12000 \mathrm{rpm}$ at $4^{\circ} \mathrm{C}$. The supernatant was collected and added to 2x SDS sample buffer in a 1:1 ratio and stored at $-20^{\circ} \mathrm{C}$ until needed.

\subsection{Haematoxylin and Eosin (H\&E) Staining}

$\mathrm{H} \& \mathrm{E}$ was used to examine tissue morphology and determine cell type and tumour areas. Slides were placed into a staining rack and deparaffinised by immersion in two changes of histolene 


\section{CHAPTER 2}

(Fronine Pty Ltd; Scoresby, Australia) for 5 minutes each. Slides were rehydrated in decreasing grades of ethanol, washed in tap water and placed in Mayer's Haematoxylin Solution (Sigma-

Aldrich) for 3 minutes. Excess Haematoxylin was washed off in running water and placed in Scott's tap water for 2 minutes. Slides were immersed in 1\% Eosin Y Solution (Simga-Aldrich) for 1 minute and dehydrated quickly (2-3 dips) in increasing grades of ethanol. Slides were then placed into fresh histolene for 10 minutes and mounted in an organic mountant, DPex, cover-slipped and allowed to dry. Slides were viewed using the NIKON Coolscope.

\subsection{Immunohistoche mistry}

IHC was used to examine the presence, location and distribution of specific proteins in the tissues. Slides were placed into a staining rack and deparaffinised by immersion in two changes of histolene (Fronine Pty Ltd) for 5 minutes each. Slides were rehydrated in decreasing grades of ethanol, washed in distilled water and antigen retrieved using $10 \mathrm{mM}$ Citrate buffer ( $\mathrm{pH} 6)$ using the water-bath method to uncover masked antigens. Slides were placed in a container filled with citrate buffer in a $99^{\circ} \mathrm{C}$ water-bath for 30 minutes. Slides were then allowed to cool to room temperature. However, for Caspase 3, antigen retrieval using the microwave method was used where slides were heated to boiling point in a sharp Carousel TM R-350J microwave oven for 4 minutes on medium high and maintained at boiling point at a low setting for 12 minutes. Slides were then allowed to cool to room temperature.

Slides were washed in TBST using gentle agitation on a magnetic stirrer for 5 minutes. $\mathrm{A}_{2} \mathrm{O}_{2}$ peroxidase block solution (Envision Plus ${ }^{\circledR}$ System-HRP kit; Dako, Botany, Australia) was used for 10 minutes at room temperature in the dark to block endogenous peroxidases and slides were washed in TBST for another 5 minutes. Sections were incubated in $5 \%$ of the normal serum from the same animal species as the secondary antibody to prevent unrelated Igprotein interactions. For all antibodies, 5\% goat serum was used, except for PAK1 where 5\% horse serum was used, and Ki67 where Ultra V-block (Thermo Fisher Scientific) was used. 


\section{CHAPTER 2}

The primary antibody was applied to the sections (see Table 2.16) and incubated overnight at $4^{\circ} \mathrm{C}$, except for $\mathrm{Ki} 67$ which was incubated for 2 hours in a $37^{\circ} \mathrm{C}$ oven in the dark. As a negative control, sections were incubated with normal Rabbit IgG (sc-2027; Santa Cruz Biotechnology, Texas, USA) at the same protein concentration as the primary antibody. This ensures that the positive stain is not due to non-specific interactions of immunoglobulin molecules with the section.

Slides were washed in TBST to remove unbound antibody and incubated in peroxidase conjugated goat anti-rabbit secondary antibody (Envision Plus ${ }^{\circledR}$ System-HRP kit) for 1 hour. Slides were washed twice with TBST and incubated in DAB (diaminobenzidine) chromogen substrate solution ( 1 drop of DAB chromogen added to $1 \mathrm{ml}$ of DAB buffer (Envision Plus ${ }^{\circledR}$ System-HRP kit)) was applied to the slides until colour was observed for a maximum of 10 minutes. Peroxidase enzyme catalyses the substrate reaction and converts the chromogen substrate to a dark brown insoluble precipitate indicative of positive staining. Slides were washed in tap water to prevent further reaction. Slides were counterstained with Mayer's Haematoxylin Solution for 3 minutes, washed in running water, and immersed in Scotts tap water for 2 minutes. After washing in tap water, slides were dehydrated in increasing grades of ethanol. Slides were then placed into fresh histolene for 10 minutes and mounted in an organic mountant, DPex, cover-slipped and allowed to dry overnight. Slides were viewed using the NIKON Coolscope.

For quantification, 24 random fields were taken of the tumour tissue at $400 \mathrm{x}$ magnification. Cells were counted using the Image Pro-Plus 6.0 image analysis program (Media Cybernetics Inc., Silver Spring, MD) and the ratio of positive cells to the total number of cells in each field was calculated. 


\section{CHAPTER 2}

Table 2.16: Primary Antibodies used for Immunohistochemistry

Target Protein Antibody Supplier (catalogue number) Dilution

\begin{tabular}{lllc}
\hline$\alpha$ SMA & Monoclonal Rabbit & Abcam (ab32575) & $1: 200$ \\
Caspase 3 & Polyclonal Rabbit & R\&D Systems (AF835) & $1: 500$ \\
desmin & Monoclonal Rabbit & Abcam (ab32362) & $1: 200$ \\
Ki67 & Monoclonal Rabbit & Neomarker (MA5-14520) & $1: 200$ \\
PAK1 & Polyclonal Rabbit & Santa Cruz (sc-882) & $1: 200$ \\
& & & \\
\hline
\end{tabular}

\subsection{Picrosirius Red Staining}

Picrosirius red staining was used to visualise the extracellular matrix protein, collagen I. Slides were placed into a staining rack and deparaffinised by immersion in two changes of histolene (Fronine Pty Ltd) for 5 minutes each. Slides were rehydrated in decreasing grades of ethanol, washed in distilled water and incubated in picrosirius red solution $(0.1 \%$ sirius red (SigmaAldrich) in saturated aqueous solution of picric acid (Sigma-Aldrich)) for 1 hour. This enhances the birefringence of collagen fibres by sirius red. Slides were then washed in two changes of acidified water ( $0.5 \%$ acetic acid glacial (Sigma-Aldrich) in distilled water) to prevent the loss of dye, tapped dry, and dehydrated in increasing grades of ethanol. Slides were then placed into fresh histolene for 10 minutes and mounted in an organic mountant, DPex, cover-slipped and allowed to dry overnight. Slides were viewed using the NIKON Coolscope with collagen I fibres appearing 'red'. 
CHAPTER 2

\subsection{Statistical Analysis}

All quantitative data are expressed as means \pm standard error of the mean (SEM). Experiments were done in duplicate and data collated from three independent experiments. Results were analysed using student's t-test or one-way ANOVA (SPSS). Survival was analysed using the Cox regression analysis, with study stratification when necessary (SPSS). Differences between two means with $\mathrm{p}<0.05$ were considered significant. 
CHAPTER 3

Chapter 3: Glaucarubinone Reduces Pancreatic

Cancer Growth and Increases Survival by Inhibition of PAK1 and PAK4 


\section{CHAPTER 3}

\subsection{Introduction}

Therapies for pancreatic cancer are limited and are ineffective. There is a need to explore targeted therapeutic agents and understand the mechanisms that underlie the disease in the hope to find effective therapies to improve pancreatic cancer patient outcomes. Glaucarubinone has been shown to possess anti-cancer activity in solid tumours but has not been investigated in pancreatic cancer previously $(56,217)$. The natural product, glaucarubinone, a quassinoid first isolated from the seeds of Simarouba glauca, and later from numerous other species in the Simaroubaceae family, was originally developed as an antimalarial drug (157). It was found that glaucarubinone may act, at least in part, via inhibition of pathways involving PAK1 where suppression of PAK1 resulted in the death of malaria parasites (197). Glaucarubinone has also been found to extend the lifespan of $C$. elegans by downregulating PAK1 (241).

Glaucarubinone (Figure 3.1) was first discovered to have cancer growth inhibition properties in murine leukemia cells in 1977 (63). Following this discovery, studies have investigated the effects of glaucarubinone on a number of cancers including breast, ovarian, and neurofibromatosis type 2 (NF2)-deficient Schwannomas (142, 212). Elucidation of the mechanisms involved have found two transcription factors: activator protein-1 (AP-1) and NFkB as likely targets of glaucarubinone, both have been shown to require PAK1 $(15,41)$. Increased PAK1 activity has been found to increase AP-1 DNA binding activity in breast cancer cells (213) and AP-1 has been found to be involved in PAK1-mediated tissue factor (TF) expression, a cell-surface glycoprotein responsible for initiating the coagulation cascade (188). Furthermore, recent implication of PAK1 pathways by glaucarubinone was reported in colorectal cancer cells where PAK1-downstream products: hypoxia-induced factor 1a (HIF$1 \alpha$ ) and $\beta$-catenin was downregulated following treatment with glaucarubinone (94). Glaucarubinone has been well studied for its anti-cancer properties and studies have linked and suggested a possible mechanism of action through PAK1-mediated pathways. 


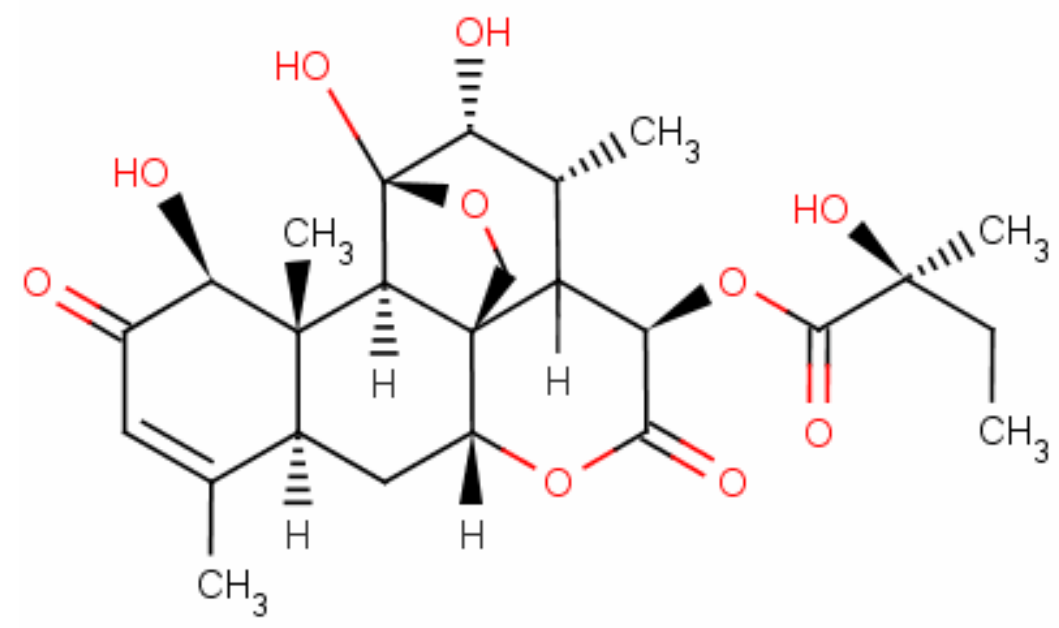

Figure 3.1 Chemical structure of glaucarubinone. Taken from (141).

The high prevalence of KRAS mutations in pancreatic cancer, evidence that PAK1 is activated by and acts downstream of KRAS, and evidence that PAK1 and PAK4 is upregulated in pancreatic cancer suggests a possible role of PAK1 and PAK4 in pancreatic cancer. Thus, to characterise the role of PAK1 and PAK4 in pancreatic cancer, this study investigated the effects of a non-specific PAK inhibitor, glaucarubinone alone and in combination with standard chemotherapy, gemcitabine, on the expression and activation of PAK1 and PAK4 in vitro. Preclinical models were utilised to investigate their role in pancreatic tumour growth in xenograft models, and murine survival in orthotopic pancreatic tumour models. 


\section{CHAPTER 3}

\subsection{Methods}

The proliferation, combination index, migration/invasion, and Western blot assays; orthotopic pancreatic cancer murine implantation, tissue processing and immunohistochemical staining procedures have been described in Chapter 2 .

\subsubsection{Subcutaneous Flank Xenograft Murine Study}

5 x $10^{6}$ PANC-1 and MiaPaCa-2 cells in $100 \mu$ DMEM were implanted subcutaneously into each flank as described in Chapter 2.11. Mice were divided into four groups (5 mice per group) as follows:

1. Control: intraperitoneal (i.p.) injection of saline every other day;

2. Glaucarubinone alone: glaucarubinone $(1 \mathrm{mg} / \mathrm{kg}$ for first week then $2 \mathrm{mg} / \mathrm{kg}$ thereafter) i.p. every other day;

3. Gemcitabine alone: gemcitabine $(20 \mathrm{mg} / \mathrm{kg})$ i.p. twice weekly; or

4. Combination of glaucarubinone and gemcitabine: following the treatment as described above.

Treatment started 7 days after tumour implantation. Mice were sacrificed on day 43, tumours extracted, weighed, and snap-frozen for protein extraction and analysis or fixed and PPFE for IHC staining as described in Chapter 2.14.

\subsubsection{Orthotopic Pancreatic Cancer Murine Study Design}

Pan02 (2.5 x $10^{5}$ cells) were suspended in $50 \mu \mathrm{L}$ of Matrigel $^{\mathrm{TM}}$ matrix solution (BD Biosciences, Franklin Lakes, USA) and implanted into the pancreatic head of C57B1/6 mice as described in Chapter 2.13. Leakage occurred in less than $5 \%$ of mice, and only mice without leakage during injection were included in experiments. Mice were monitored based on health score for up to 45 days and euthanised when a poor health score was reached. Two studies were undertaken: 


\section{CHAPTER 3}

in the first study, 48 mice were implanted with Pan02 cells and groups of 12 mice were randomly allocated to one of four groups:

1. Control: i.p. saline injection every other day;

2. Glaucarubinone alone: glaucarubinone $(2 \mathrm{mg} / \mathrm{kg})$ i.p. every other day;

3. Gemcitabine alone: gemcitabine $(40 \mathrm{mg} / \mathrm{kg})$ i.p. twice weekly; or

4. Combination of glaucarubinone and gemcitabine: following the treatment as described above.

In the second study, 30 mice were implanted with Pan02 cells, with 13 mice randomly allocated to either the gemcitabine alone or combination treatment group, with the remaining 4 mice allocated to the control group. A collated Kaplan-Meier survival curve was plotted (SPSS). Treatment started 7 days after tumour implantation, at a time when discrete tumours had formed. Identical experiments were undertaken using the other murine pancreatic cancer cell line, LM-P, (1 x $10^{6}$ cells $)$ which were suspended in $50 \mu \mathrm{L}$ of Matrigel $^{\mathrm{TM}}$ matrix solution and implanted into 48 mice. Mice were divided into control, glaucarubinone alone, gemcitabine alone, and combination of glaucarubinone and gemcitabine treatment groups, in the same manner as the first study using Pan02 cells. 


\subsection{Results}

This study was a continuation of work completed as part of an Honours degree. As such, in vitro data of the effects of glaucarubinone and gemcitabine on PANC-1, MiaPaCa-2 and Pan02 cell proliferation, migration/invasion will not be presented here, but the $\mathrm{IC}_{50}$ values will be included for comparison.

\subsubsection{Glaucarubinone alone and gemcitabine alone inhibited pancreatic cancer cell proliferation}

The effect of the inhibitors, glaucarubinone and gemcitabine, as single treatment agents was tested on pancreatic cancer cell proliferation. Glaucarubinone inhibited the proliferation of the additional three pancreatic cancer cell lines tested: AsPC-1, BxPC-3, and LM-P; in a dosedependent manner (Fig. 3.3.1A). The $\mathrm{IC}_{50}$ values were $265 \mathrm{nM}$ for AsPC-1, 60nM for BxPC-3, and $1465 \mathrm{nM}$ for LM-P in addition to the other three pancreatic cancer cell lines: $340 \mathrm{nM}$ for PANC-1, 65nM for MiaPaCa-2, and 965nM for Pan02 (Table 3.3.1).

Similarly, gemcitabine inhibited the proliferation of the additional three pancreatic cancer cell lines tested in a dose-dependent manner (Fig. 3.3.1B). The $\mathrm{IC}_{50}$ values were $20 \mathrm{nM}$ for AsPC1, $5 \mathrm{nM}$ for BxPC-3, and 15nM for LM-P in addition to the other three pancreatic cancer cell lines: 40nM for PANC-1, 4nM for MiaPaCa-2, and 100nM for Pan02 (Table 3.3.1). 

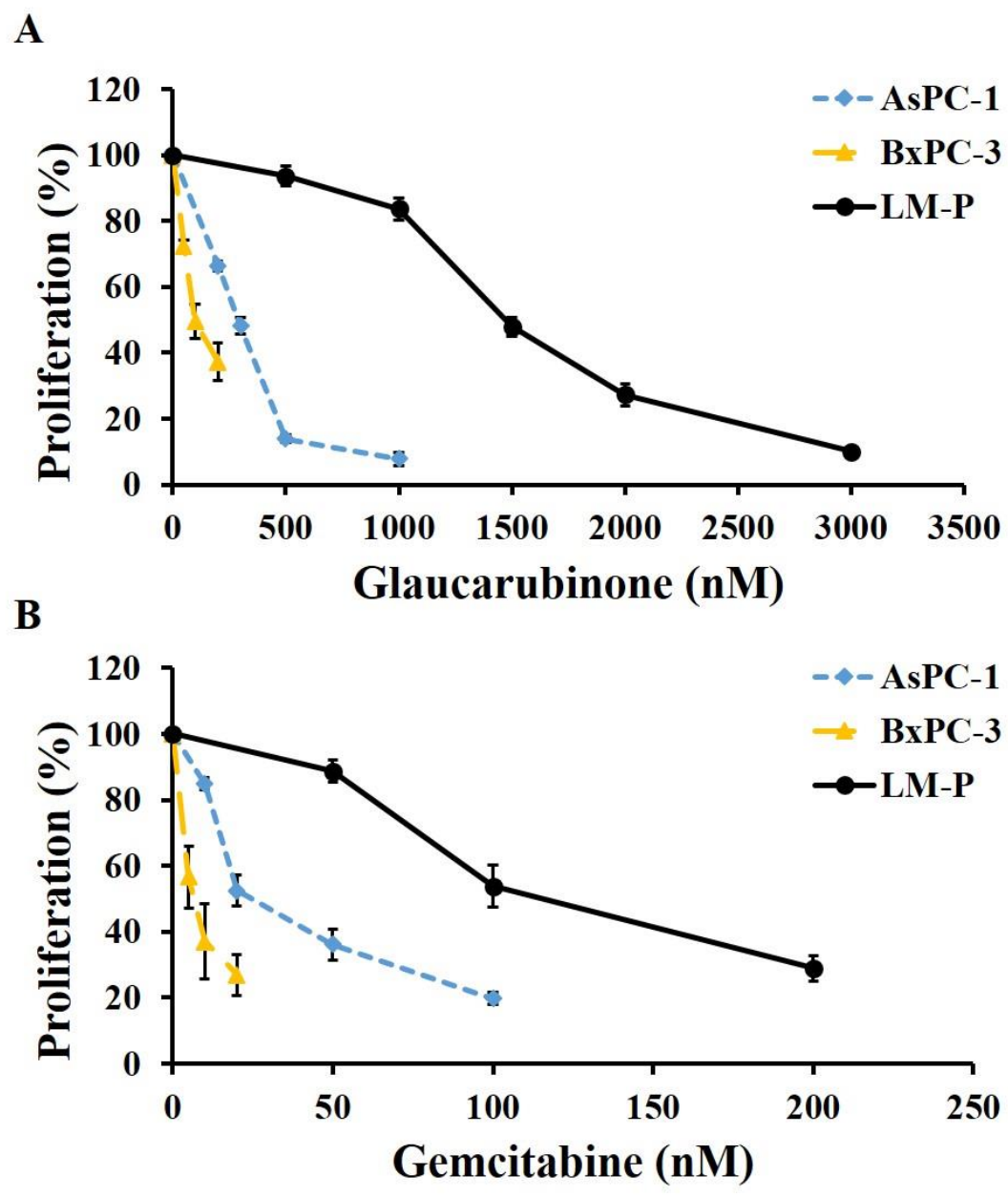

Figure 3.3.1 Glaucarubinone and gemcitabine inhibited pancreatic cancer proliferation in a dose dependent manner. Human pancreatic cancer cell lines: AsPC-1 and BxPC-3; and the murine pancreatic cancer cell line: LM-P, were treated with glaucarubinone (A) or gemcitabine (B) and proliferation measured using ${ }^{3} \mathrm{H}$-thymidine incorporation assay. 
Table 3.3.1: Proliferation $\mathrm{IC}_{50}$ values for glaucarubinone and gemcitabine

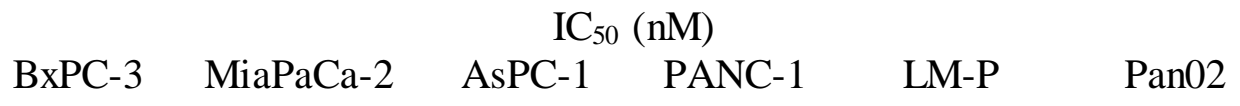

$\begin{array}{lcccccc}\text { Glaucarubinone } & 60 \pm 10 & 65 \pm 20 & 265 \pm 15 & 340 \pm 90 & 1465 \pm 65 & 965 \pm 290 \\ \text { Gemcitabine } & 5 \pm 2 & 45 \pm 3 & 20 \pm 3 & 40 \pm 6 & 15 \pm 2 & 100 \pm 2\end{array}$

\subsubsection{Glaucarubinone inhibited pancreatic cancer cell migration/invasion}

The effect of glaucarubinone on pancreatic cancer cell migration/invasion of the additional murine pancreatic cancer cell line, LM-P, was tested. Glaucarubinone inhibited the migration and invasion of LM-P cells in a dose-dependent manner (Fig. 3.3.2). The $\mathrm{IC}_{50}$ value was $120 \mathrm{nM}$ for LM-P in addition to $44 \mathrm{nM}$ for MiaPaCa-2, 210nM for PANC-1, and 220nM for Pan02 (Table 3.3.2).

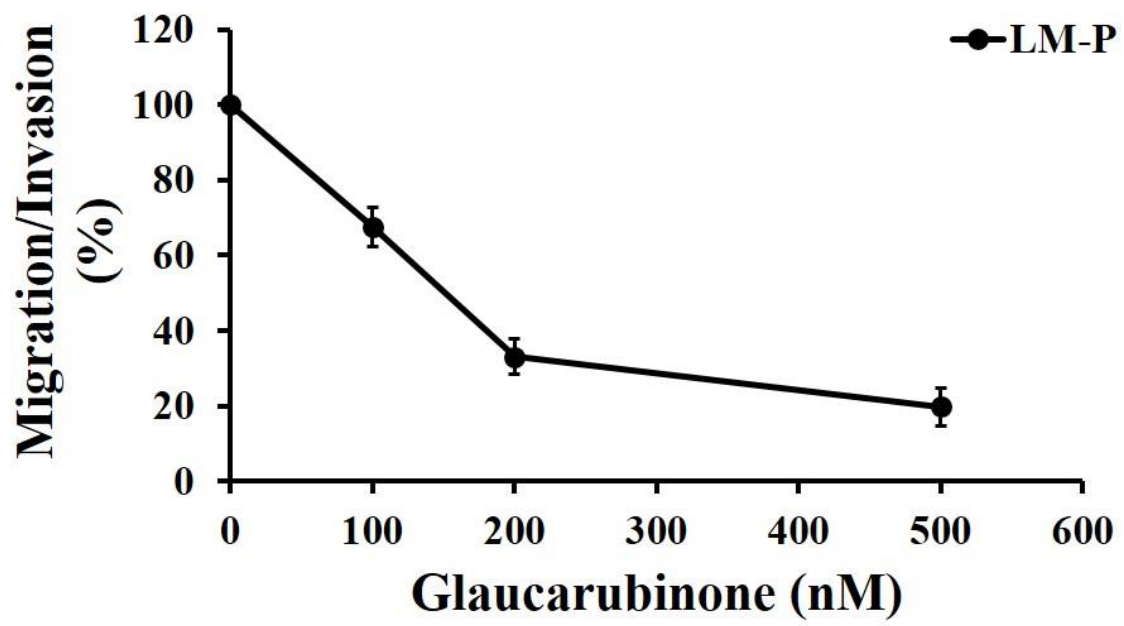

Figure 3.3.2 Glaucarubinone inhibited pancreatic cancer migration/invasion in a dose dependent manner. Murine pancreatic cancer cell line, LM-P, were treated with glaucarubinone and migration/invasion measured using the Transwell Boyden Chamber assay. 
Table 3.3.2: Migration and invasion $\mathrm{IC}_{50}$ values for glaucarubinone

$$
\begin{array}{lll}
\mathrm{IC}_{50}(\mathrm{nM}) & \\
\text { MiaPaCa-2 } & \text { PANC-1 } & \text { LM-P }
\end{array}
$$

\begin{tabular}{lllll}
\hline Glaucarubinone & $44 \pm 23$ & $210 \pm 20$ & $120 \pm 10$ & $220 \pm 130$
\end{tabular}

\subsubsection{Inhibition of glaucarubinone correlated with PAK1 activity}

The six pancreatic cancer cell lines had varying expression of phosphorylated PAK1 (pPAK1), PAK1, active form of PAK4 (pPAK4), and PAK4 (Fig. 3.3.3A-B). The $\mathrm{IC}_{50}$ values for inhibition of proliferation by glaucarubinone varied among the six pancreatic cancer cell lines. BxPC-3 and MiaPaCa-2 was most sensitive to glaucarubinone with the lowest $\mathrm{IC}_{50}$ values, while Pan02 and LM-P was least sensitive to glaucarubinone with the highest $\mathrm{IC}_{50}$ values (Table 3.3.1). Trends were observed in the active form of PAK1 (pPAK1) to the $\mathrm{IC}_{50}$ values as BxPC-3 and MiaPaCa-2 had the lowest expression of the active form of PAK1 (pPAK1), while Pan02 and LM-P had the highest expression of pPAK1 with an R-square value of 0.9839 (Fig. 3.3.3C). No observable trend was found with PAK1, pPAK4 or PAK4 expression. The matching trend of $\mathrm{IC}_{50}$ values with PAK1 activity suggests that glaucarubinone may be acting through PAK1 mediated pathways. 


\section{CHAPTER 3}
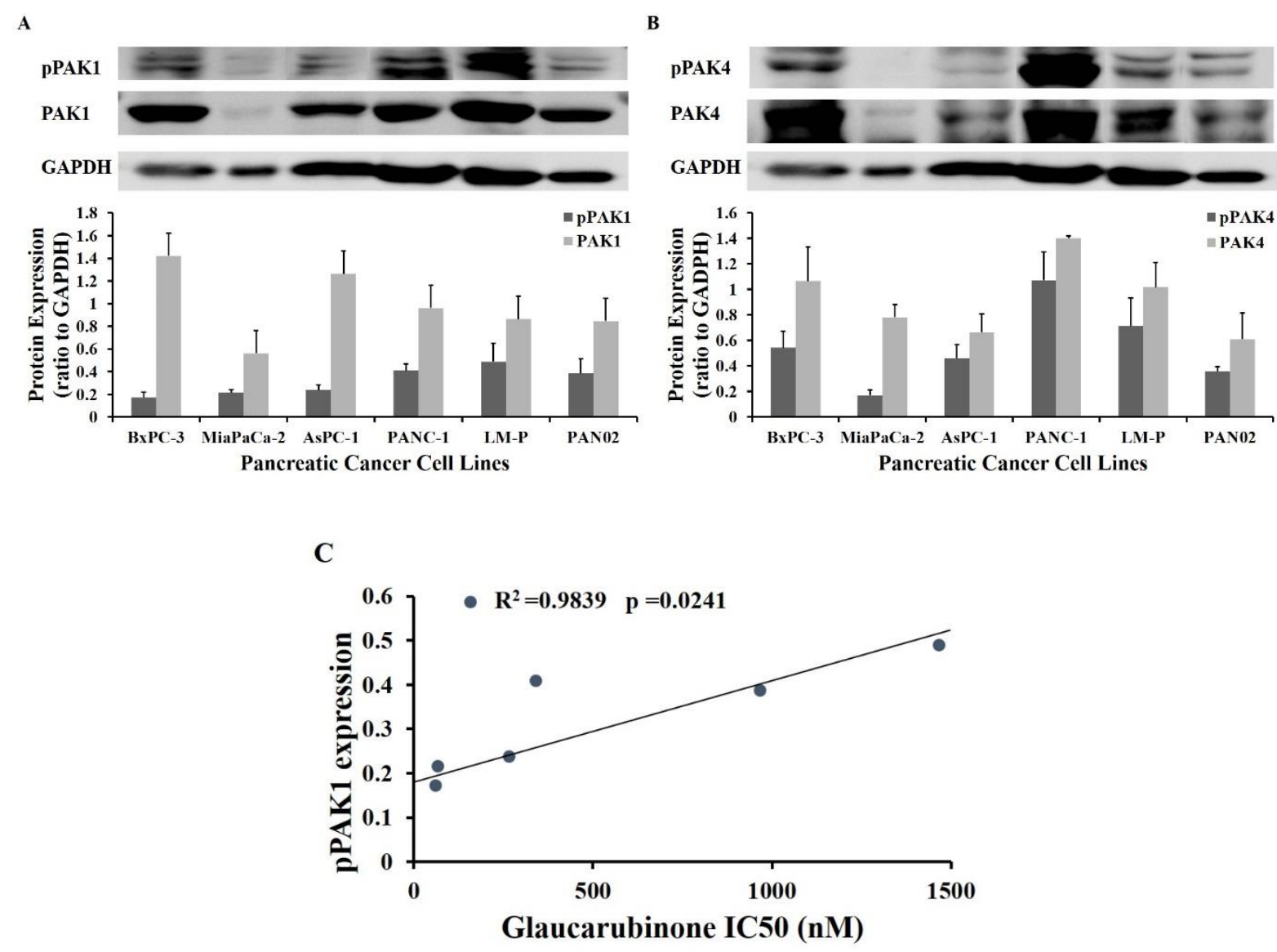

Figure 3.3.3 PAK1 and PAK4 is expressed in all pancreatic cancer cell lines tested and glaucarubinone inhibition correlated with PAK1 activity. Varying expressions of active form of PAK1 (pPAK1) (A), PAK1 (A), active form of PAK4 (pPAK4) (B), and PAK4 (B) was found in the 6 pancreatic cancer cell lines tested: 4 human pancreatic cancer cell lines: BxPC-3, MiaPaCa-2, AsPC-1, and PANC-1; and 2 murine pancreatic cancer cell lines: LM-P and Pan02. Glaucarubinone proliferation inhibition $\mathrm{IC}_{50}$ correlated significantly with pPAK1 expression $(\mathrm{C})$. 


\subsubsection{Glaucarubinone and gemcitabine synergistically inhibited LM-P pancreatic cancer} cell growth

The combined effect of glaucarubinone and gemcitabine on proliferation were measured by incubation of LM-P cells with increasing concentrations of gemcitabine, alone or in combination with glaucarubinone, at a concentration close to the $\mathrm{IC}_{50}$ value given in Table 3.3.1. A further reduction of proliferation was observed when the cells were treated with the combination of glaucarubinone and gemcitabine, compared to gemcitabine alone (Fig. 3.3.4A). The combined effect of glaucarubinone and gemcitabine was shown to be synergistic (combination index < 1) using the Chou-Talalay method (Fig. 3.3.4B). This was similar to the results from the MiaPaCa-2, PANC-1, and Pan02 cell lines. These results demonstrated that glaucarubinone and gemcitabine synergistically inhibited pancreatic cancer cell growth.

A

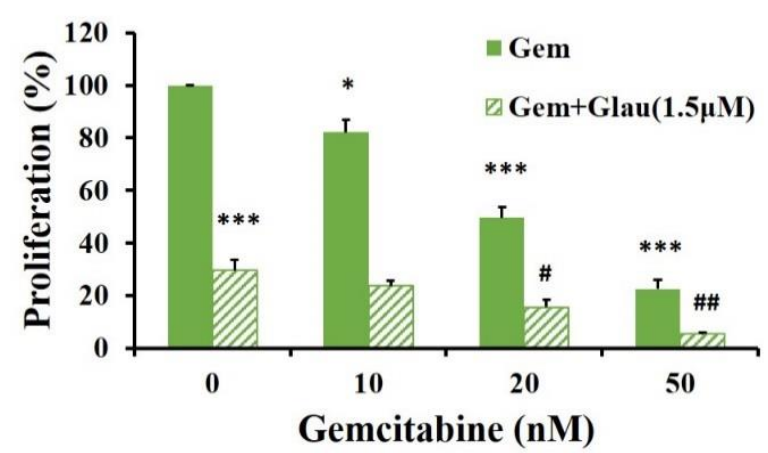

B

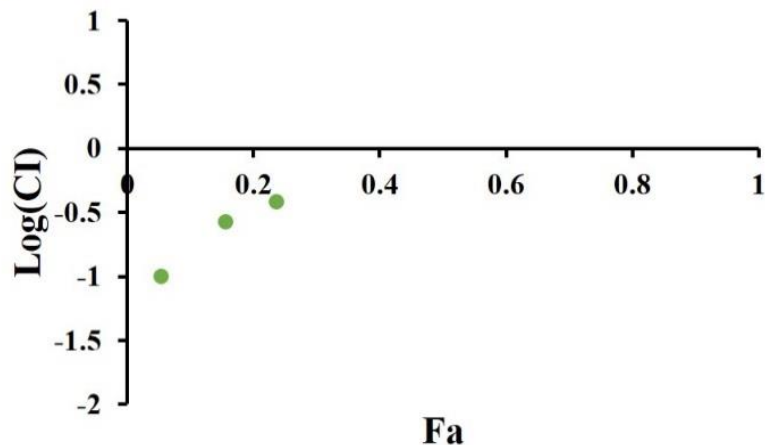

Figure 3.3.4 Glaucarubinone synergised with gemcitabine to inhibit LM-P proliferation. The effects of gemcitabine alone (Gem, solid bars), and gemcitabine after 20 hours pretreatment with glaucarubinone (Gem + Glau, striped bars), on proliferation of LM-P murine pancreatic cancer cells were assessed by ${ }^{3} \mathrm{H}$-thymidine incorporation assay (A). The concentration of glaucarubinone used was based on the $\mathrm{IC}_{50}$ value determined in Chapter 3.3.1. The combination index (CI), calculated by the Chou-Talalay method, was used to determine the mechanism of action of glaucarubinone and gemcitabine (B). A CI value $<1$ indicates 


\section{CHAPTER 3}

synergistic inhibition. ${ }^{*} \mathrm{p}<0.05,{ }^{* * *} \mathrm{p}<0.001$, compared to untreated cells. $\# \mathrm{p}<0.05, \# \# \mathrm{p}<0.01$ compared to the corresponding gemcitabine treatment.

\subsubsection{Inhibition of pancreatic cancer cell growth by glaucarubinone and gemcitabine associated with a reduction in active PAK1 and PAK4}

The effects of the glaucarubinone and gemcitabine on the expression and activity of PAK1 and PAK4 were determined as a possible mechanism for the observed inhibition of pancreatic cancer cell growth. The human pancreatic cancer cell line MiaPaCa-2 was treated with increasing concentrations of glaucarubinone and the total amounts of active (pPAK1 and pPAK4) and total (PAK1 and PAK4) protein expression was measured by Western blotting. Glaucarubinone significantly decreased the active amount of PAK1 and PAK4 at 50nM and $100 \mathrm{nM}$ with no effect on the corresponding total protein expression (Fig. 3.3.5A-B).

The human pancreatic cancer cell lines, PANC-1 and MiaPaCa-2, were treated with gluacarubinone and/or gemcitabine at a concentration close to their $\mathrm{IC}_{50}$ value (Table 3.3.1). The total amounts of active (pPAK1 and pPAK4) and total (PAK1 and PAK4) protein expression was measured. In MiaPaCa-2 cells glaucarubinone significantly decreased the amount of both pPAK1 (Fig. 3.3.5C) and pPAK4 (Fig. 3.3.5D) without affecting the expression of either PAK1 or PAK4 while gemcitabine and had no effect on the amount of either active or total PAK1 or PAK4. However, the combination of glaucarubinone and gemcitabine further decreased the amounts of both pPAK1 and pPAK4 (Fig. 3.3.5C-D). Similar results were obtained in PANC-1 cells, however, single treatment of glaucarubinone did not reach significance in reducing active PAK1 or PAK4, although was significant in the combination treatment of glaucarubinone and gemcitabine (Fig. 3.3.5E-F). These data suggested that 
CHAPTER 3

glaucarubinone and gemcitabine synergistically reduced pancreatic cancer cell growth by reducing the amounts of active PAK1 and PAK4. 
A

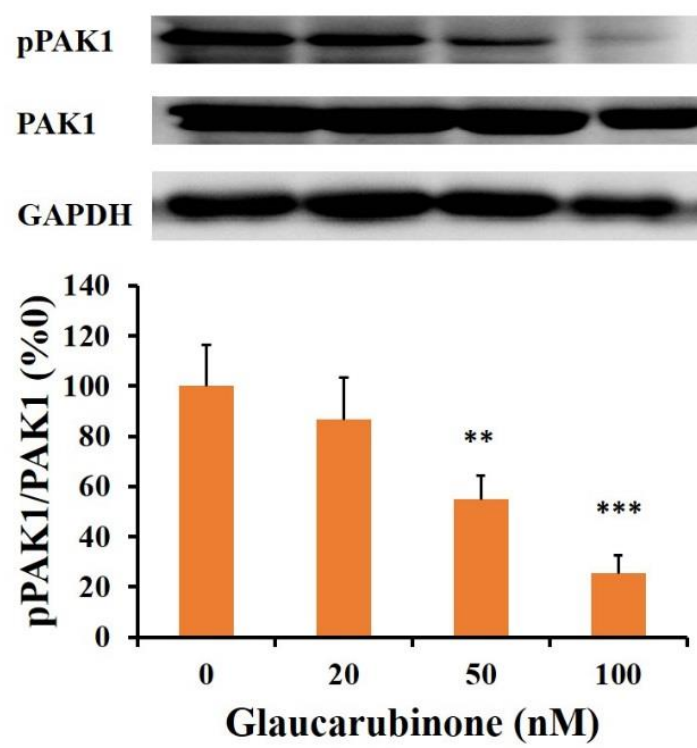

C
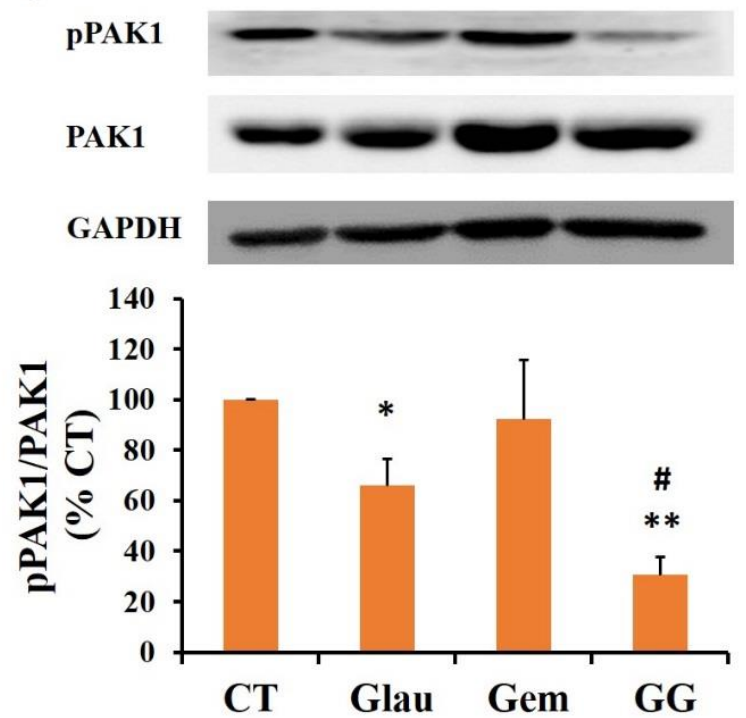

E
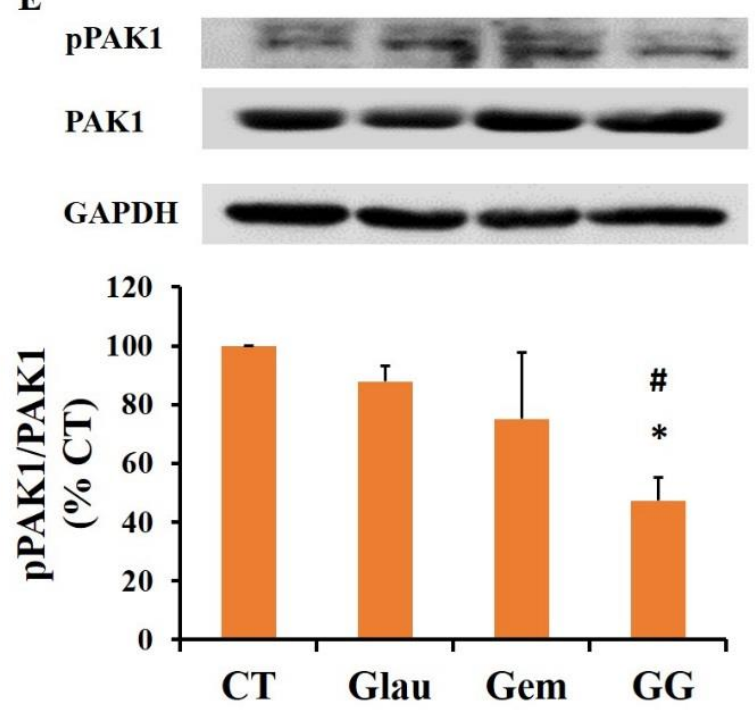

B
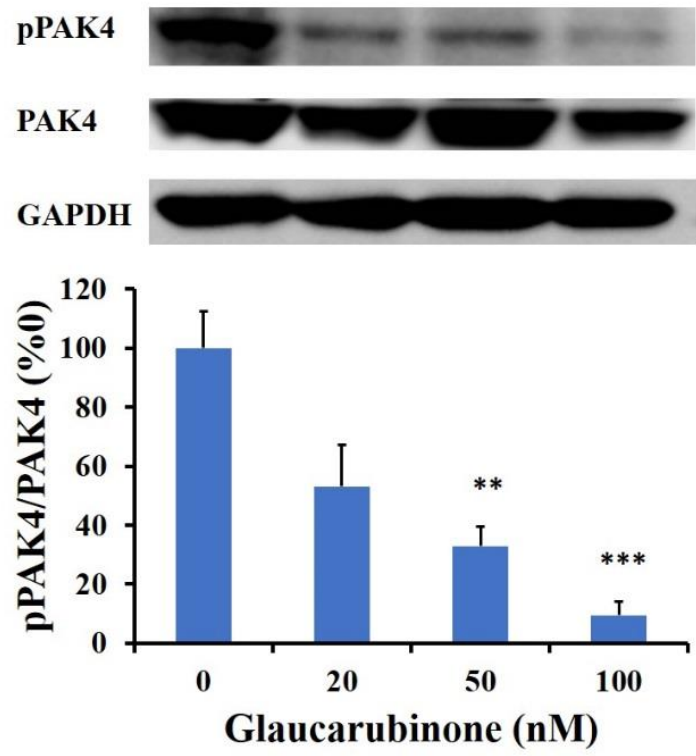

D
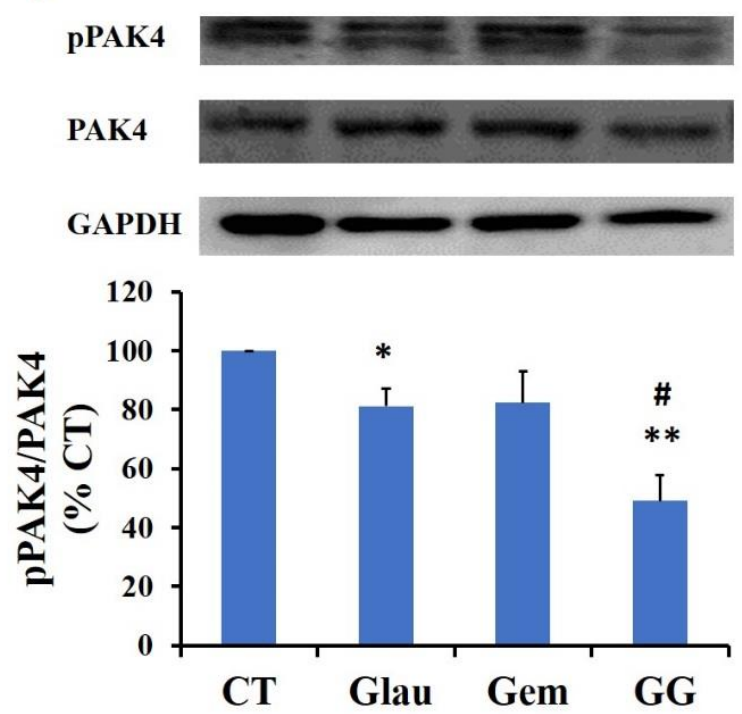

F
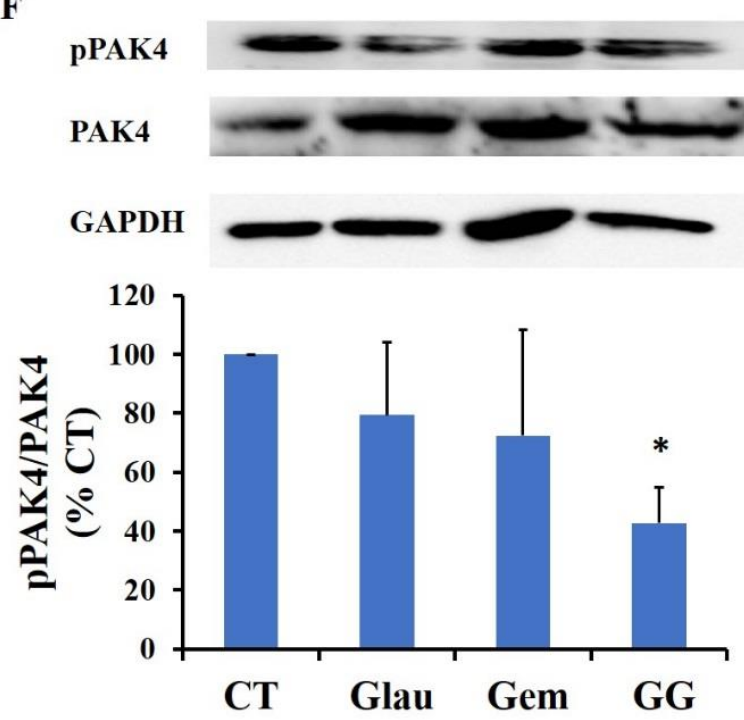
Figure 3.3.5 Glaucarubinone and gemcitabine synergistically reduced the amounts of active PAK1 and PAK4 in vitro. The effects of glaucarubinone (Glau) or gemcitabine (Gem) alone, or in combination (GG), on the amounts of active PAK1 (orange graphs) or PAK4 (blue graphs) were determined in MiaPaCa-2 (A, B, C, D) or PANC-1 (E, F) cells by Western blotting. Glaucarubinone dose-dependently decreased PAK1 and PAK4 activity (A, B). The combination of glaucarubinone and gemcitabine further reduced PAK1 and PAK4 activity in MiaPaCa-2 cells (C, D) and PAK1 activity in PANC-1 cells (E). Although glaucarubinone and gemcitabine treatment did not decrease PAK4 activity in PANC-1 cells, the combination of glaucarubinone and gemcitabine resulted in a significant decrease compared to control (F). The concentration of glaucarubinone used was based on the $\mathrm{IC}_{50}$ value determined in Chapter 3.3.1 which was $50 \mathrm{nM}$ and $300 \mathrm{nM}$ for MiaPaCa-2 and PANC-1 cells, respectively. The concentration of gemcitabine used was based on the $\mathrm{IC}_{50}$ value determined in Chapter 3.3.1 and was 50nM for both MiaPaCa-2 and PANC-1 cells. The values from untreated control cells $(0$ or $\mathrm{CT})$ were taken as $100 \%{ }^{*} \mathrm{p}<0.05,{ }^{*} \mathrm{p}<0.01, * * * \mathrm{p}<0.001$ compared to control. $\# \mathrm{p}<0.05$ compared to glaucarubinone treatment.

\subsubsection{Glaucarubinone and gemcitabine inhibited pancreatic cancer xenograft growth in vivo by decreasing proliferation.}

The effect of glaucarubinone and gemcitabine on pancreatic cancer growth in vivo was investigated by injecting MiaPaCa- 2 and PANC- 1 cells subcutaneously into the flank of SCID mice. The tumour take rate was $100 \%$ for both cell lines. The mice were treated with glaucarubinone and gemcitabine either alone or in combination as described in Chapter 3.2.1. There was no reported toxicity in any of the mice throughout the study. From day 17, the tumour volume of the MiaPaCa-2 xenografts was significantly decreased by treatment with either glaucarubinone alone or gemcitabine alone or combination $(\mathrm{p}<0.05$ for alone treatment, 


\section{CHAPTER 3}

$\mathrm{p}<0.01$ for combination treatment, Fig. 3.3.6A). From day 28, the combination treatment of glaucarubinone and gemcitabine decreased tumour volume significantly more than the gemcitabine treatment alone $(\mathrm{p}<0.01)$, but not more than glaucarubinone treatment alone. Similarly, by the end of the endpoint, the tumour weights of MiaPaCa-2 xenografts were significantly reduced by glaucarubinone or gemcitabine alone to $28 \%$ and $44 \%$, respectively, of control tumours (Fig. 3.3.6B). The combined treatment with glaucarubinone and gemcitabine also reduced the tumour weight to $20 \%$ of control, and the reduction was significantly greater than for gemcitabine-treated tumours $(\mathrm{p}=0.035)$, but not for glaucarubinone-treated tumours. For PANC-1 xenografts, neither glaucarubinone nor gemcitabine alone had any significant effect on the tumour growth or tumour weight (Fig. 3.3.6C-D). However, the combined treatment of glaucarubinone and gemcitabine significantly reduced the tumour volume from day $32(\mathrm{p}<0.05)$ and at the endpoint, the tumour weight was $37 \%$ of control tumours $(\mathrm{p}=0.013)$.

Proliferation and apoptosis of the MiaPaCa-2 xenograft tumours were measured by IHC. Proliferation in tumours treated with the combination of glaucarubinone and gemcitabine was significantly reduced compared to untreated control tumours ( $75 \%$ of control, $\mathrm{p}=0.020)$ although there was no significant difference in proliferation between untreated control tumours and tumours treated with single treatment of either glaucarubinone or gemcitabine (Fig. 3.3.6E). There was no significant difference in apoptosis between tumours from the treatment groups compared to control (Fig. 3.3.6F). These results indicate that glaucarubinone and gemcitabine synergistically inhibited pancreatic cancer growth in vivo by reducing proliferation rather than inducing apoptosis. 
A

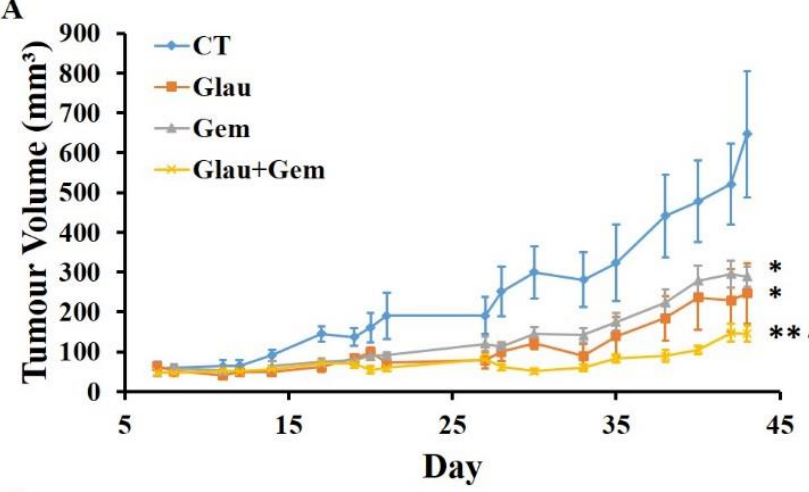

C

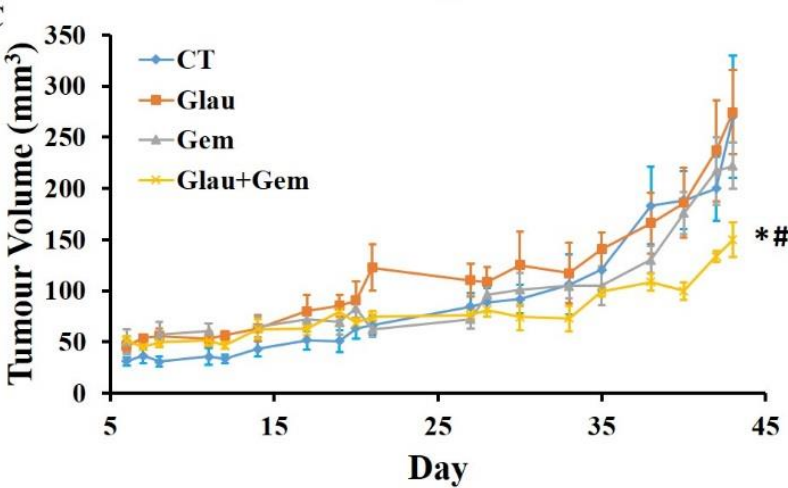

B

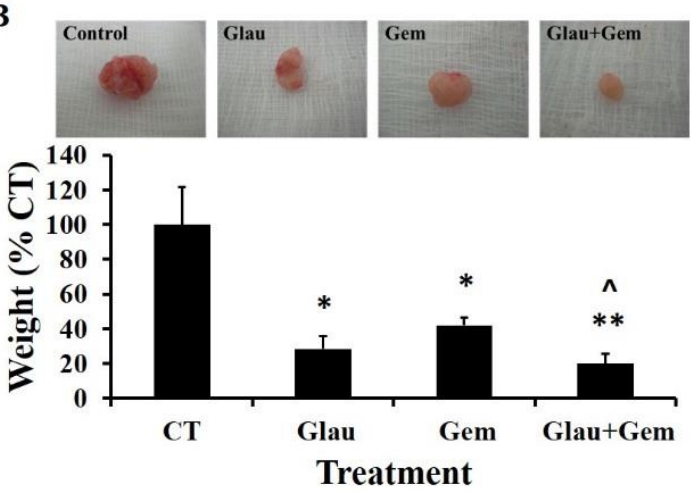

D
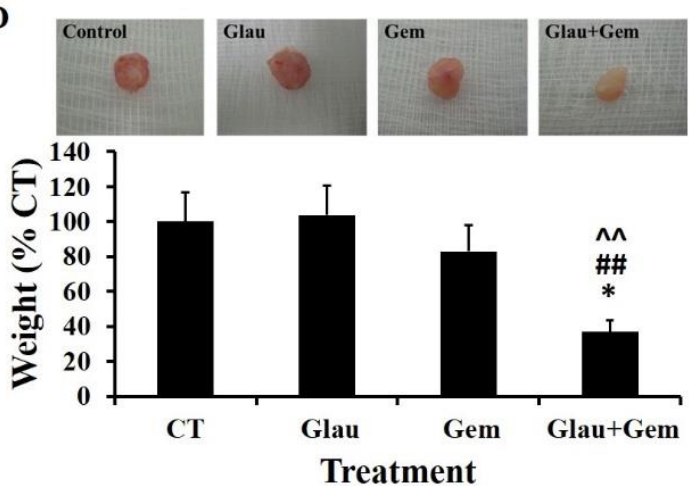

E

IgG

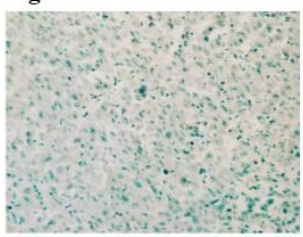

CT

Glau
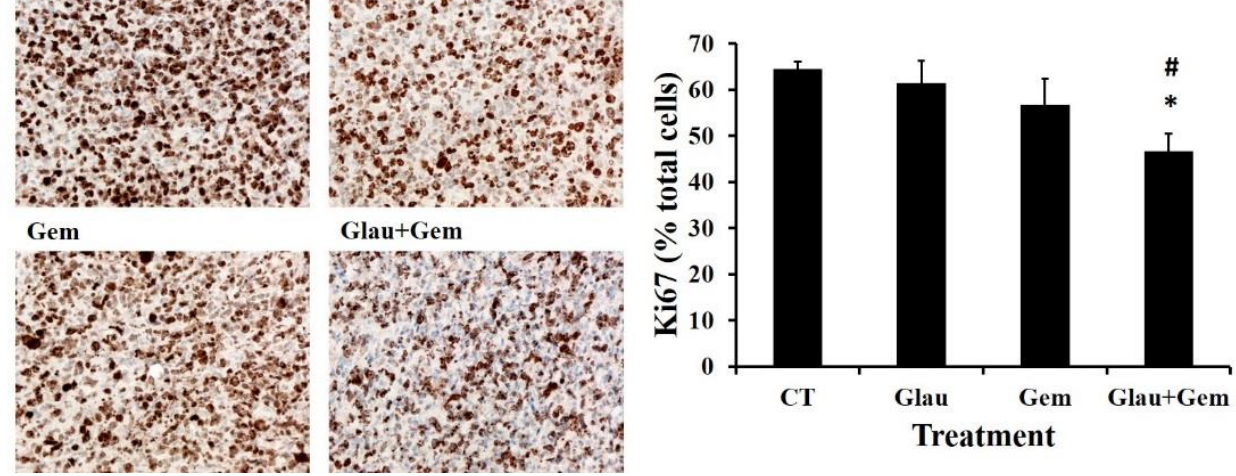

F

IgG

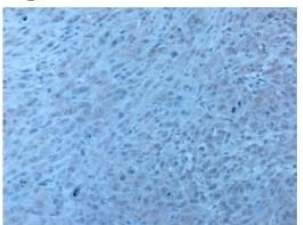

CT

Glau

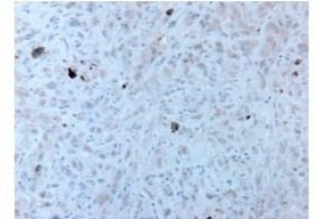

Gem
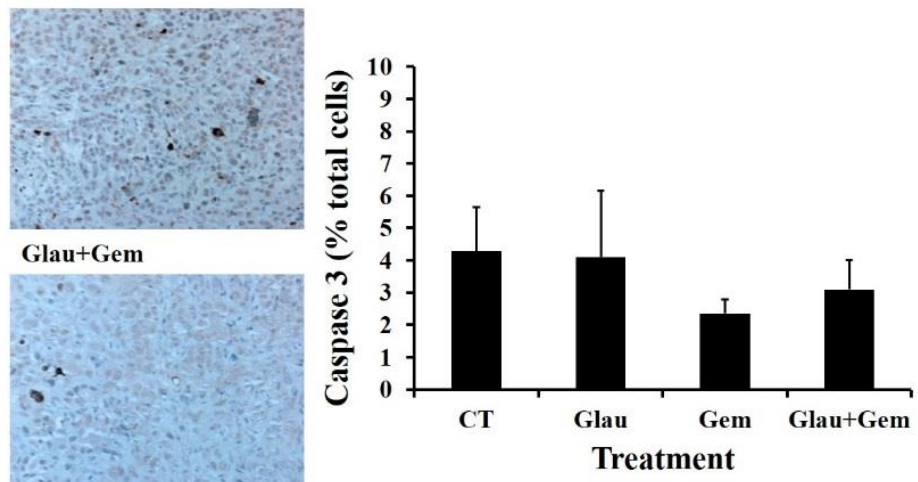
Figure 3.3.6 Glaucarubinone and gemcitabine synergistically inhibited pancreatic cancer tumour growth in vivo by decreasing proliferation. MiaPaCa-2 and PANC-1 cells were injected subcutaneously into flanks of SCID mice. The animals were treated with saline (CT), glaucarubinone (Glau), gemcitabine (Gem), or the combination (Glau + Gem). Both single treatment of glaucarubinone and gemcitabine inhibited the growth of $\mathrm{MiaPaCa}-2$ tumours and weight (A, B), but not in PANC-1 tumours (C, D). The combination of glaucarubinone and gemcitabine further decreased the growth and weight of MiaPaCa-2 tumours, and also inhibited the growth and of PANC-1 tumours. Proliferation and apoptosis in the excised tumours was measured by Ki67 and caspase 3 immunohistochemistry, respectively, and the ratios of positively stained cells to total cells were calculated. Anti-rabbit IgG was used as an isotype control. The combination of glaucarubinone and gemcitabine decreased tumour cell proliferation with decreased Ki67 staining compared to control (E). Treatments did not significant change tumour cell apoptosis with no difference in caspase 3 staining in any of the treatment groups $(\mathrm{F}){ }^{*} \mathrm{p}<0.05, * * \mathrm{p}<0.01$ compared to control (at endpoint for $\mathrm{A} \& \mathrm{C}$ ). \# $\mathrm{p}<0.05$, \#\# $\mathrm{p}<0.01$ compared to glaucarubinone treatment (at endpoint for $A \& C$ ). ${ }^{\wedge} p<0.01$ compared to gemcitabine treatment (at endpoint for A\&C).

\subsubsection{Glaucarubinone and gemcitabine decreased the amounts of active PAK1 and PAK4} in vivo

The effects of glaucarubinone and gemcitabine on the expression and activity of PAK1 and PAK4 on the xenograft tumours was investigated. As with the results from Chapter 3.3.5, glaucarubinone alone decreased the amounts of active PAK1 (pPAK1) in both MiaPaCa-2 and PANC-1 tumours, without affecting the expression of total PAK1 (Fig. 3.3.7A\&C). Combined treatment with glaucarubinone and gemcitabine further reduced the amounts of active PAK1 in both MiaPaCa-2 and PANC-1 tumours. However, in contrast to the in vitro results, only 
treatment with glaucarubinone and gemcitabine in combination significantly reduced the amounts of active PAK4 in both MiaPaCa-2 and PANC-1 tumours (Fig. 3.3.7B\&D). Neither glaucarubinone nor gemcitabine alone affected the amount of active PAK4 in MiaPaCa-2 tumours (Fig. 3.3.7B). Interestingly, gemcitabine reduced the amount of active PAK4 in PANC-1 tumours, while glaucarubinone had no significant effect on the amount of active PAK4 in PANC-1 tumours (Fig. 3.3.7D). These results suggested that glaucarubinone and gemcitabine synergistically reduced pancreatic cancer growth in vivo, at least partially through a reduction in the amounts of active PAK1 and PAK4. 
A

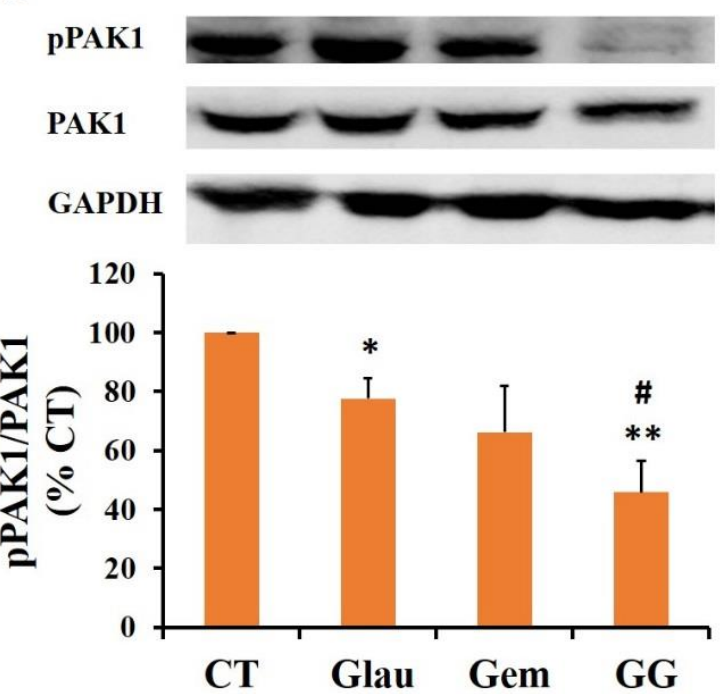

C
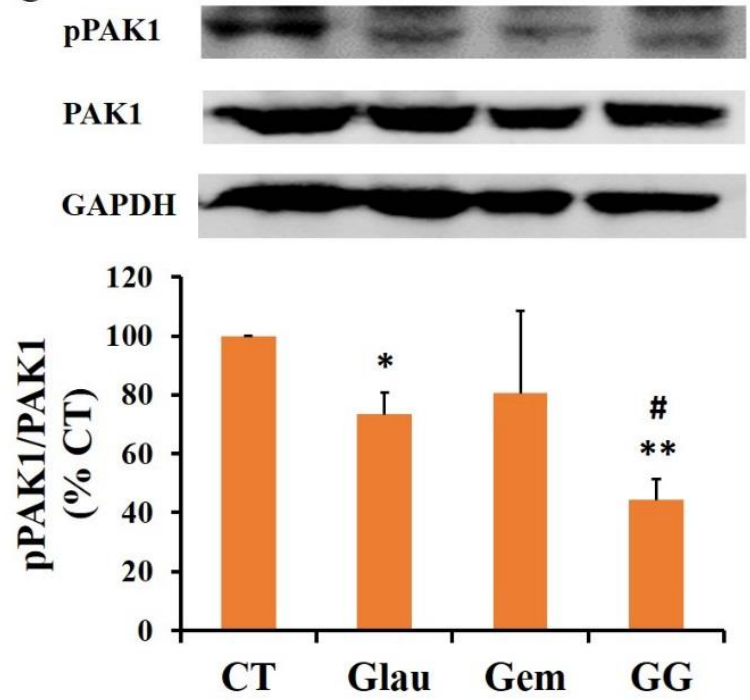

B
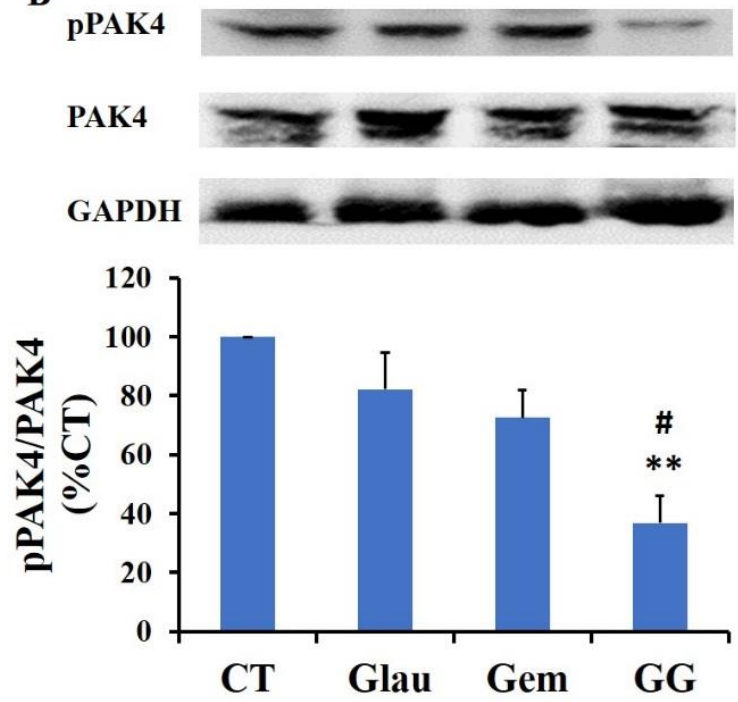

D
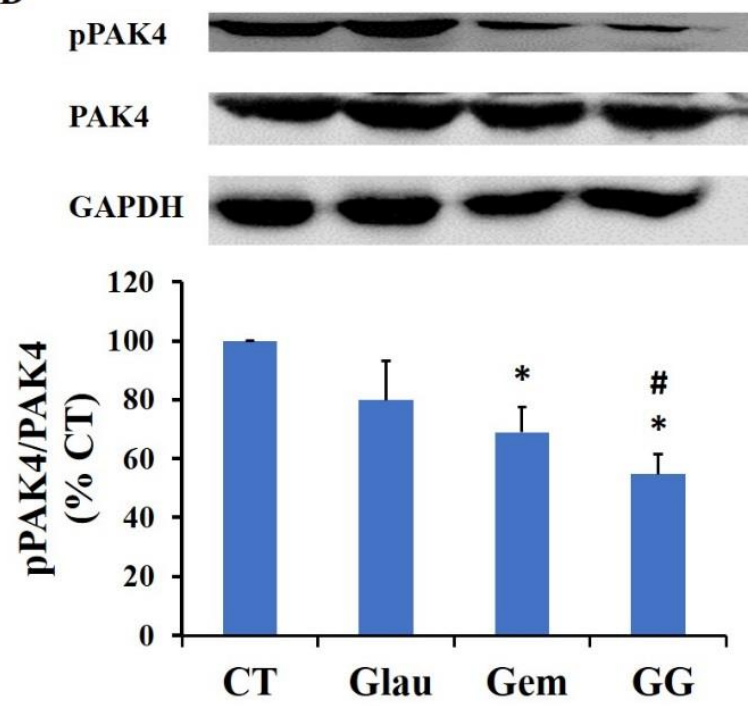

Figure 3.3.7 Glaucarubinone and gemcitabine synergistically reduced the amounts of active PAK1 and PAK4 in vivo. MiaPaCa-2 and PANC-1 cells were injected subcutaneously into flanks of SCID mice. The animals were treated with saline (CT), glaucarubinone (Glau), gemcitabine (Gem), or the combination (GG). The amounts of active PAK1 (orange graphs) or PAK4 (blue graphs) were determined in excised MiaPaCa-2 (A, B) or PANC-1 (C, D) tumours by Western blotting. The combination of glaucarubinone and gemcitabine further reduced PAK1 and PAK4 activity in MiaPaCa-2 cells (A, B) and PANC-1 cells (C, D). The values from untreated control tumours (CT) were taken as $100 \%$. ${ }^{*} \mathrm{p}<0.05,{ }^{* *} \mathrm{p}<0.01$ compared to control. \# $\mathrm{p}<0.05$ compared to glaucarubinone treatment. 


\section{CHAPTER 3}

\subsubsection{Glaucarubinone and gemcitabine treatment improved survival compared to}

\section{gemcitabine alone}

The effect of glaucarubinone on murine survival was investigated in an immunocompetent orthotopic murine model. In this syngeneic model, murine pancreatic cancer cells were implanted into the head of the murine pancreas, with formation of tumours and metastases representative of the clinical situation. Uncontrolled tumour growth in this model results in jaundice, duodenal obstruction and liver metastases.

Pan02 cell lines grew in $100 \%$ of cases and were used for survival analysis. The results of two separate studies using Pan02 cells were collated and survival curves plotted (Fig. 3.3.8). There was no reported toxicity in any of the mice throughout the studies. The median survival for the untreated control animals was 19 days. No significant improvement of survival was observed with treatment of glaucarubinone alone. Gemcitabine treatment alone resulted in improved survival compared to both the control and glaucarubinone treatment groups, with an increased median survival of 33 days $(\mathrm{p}<0.05)$ and $24 \%$ survival at 45 days following tumour induction. The combination of glaucarubinone and gemcitabine had the greatest improvement on survival with $51 \%$ of animals surviving to 45 days following tumour induction. The combination treatment significantly improved survival compared to gemcitabine alone with an odds ratio of 2.1 (95\% confidence $1.0-4.4 ; \mathrm{p}=0.046$; Table 3.3 .8 ). Drug specific toxicity was not observed in this study at the doses tested. These results indicate that glaucarubinone and gemcitabine synergistically increased pancreatic cancer murine survival in vivo. 


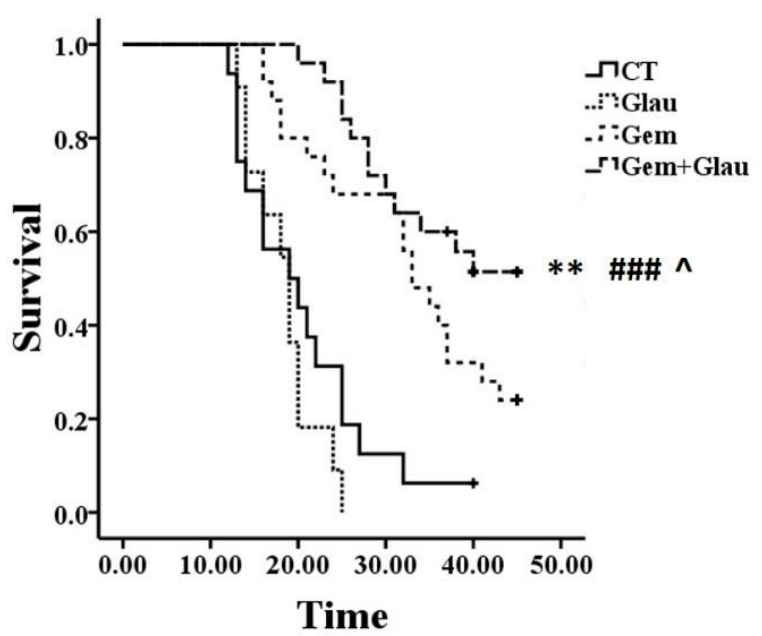

Figure 3.3.8. Glaucarubinone combined with gemcitabine significantly improved survival in a syngeneic orthotopic murine model. Pan02 cells were implanted in the head of the pancreas of $\mathrm{C} 57 \mathrm{Bl} / 6$ mice, and treatment commenced seven days thereafter. The survival curves of the animals group are presented using the Kaplan-Meier method (A). Control animals (CT) had a median survival of 20 days and no significant improvement in survival was noted when compared to glaucarubinone (Glau) treatment alone. Treatment with gemcitabine (Gem) or the combination (Gem+Glau) significantly improved survival compared to the control group. The combination treatment group itself had significantly improved survival compared to the gemcitabine alone group. Stratified cox regression survival analysis: $* * \mathrm{p}<0.01$ compared to control, \#\#\# p<0.001 compared to glaucarubinone treatment, ${ }^{\wedge} \mathrm{p}<0.05$ compared to gemcitabine treatment. 


\section{CHAPTER 3}

Table 3.3.8: Cox regression survival analysis of treatment of glaucarubinone and gemcitabine in a mouse orthotopic pancreatic cancer model

\begin{tabular}{llll} 
Treatment & Rates Ratio & $95 \%$ CI & p-value \\
\hline Control & 5.03 & $1.92-13.21$ & 0.001 \\
Glaucarubinone & 6.23 & $2.28-17.17$ & $<0.001$ \\
Gemcitabine & 2.10 & $1.01-4.36$ & 0.046 \\
Glaucarubinone + & $1.00($ ref $)$ & & \\
Gemcitabine & &
\end{tabular}

The overall statistics for the stratified Cox regression analysis were: $\chi 2(3)=17.5, p=0.001$

\subsubsection{LM-P murine pancreatic cancer cells failed to grow in $\mathrm{C} 57 \mathrm{~B} 1 / 6$ mice}

Similar to Chapter 3.3.8, LM-P cells were implanted into the head of pancreas of C57B1/6 mice and treated with glaucarubinone and gemcitabine. No LM-P tumours were observed at the endpoint in the C57BL/6 mice, in control or treated animals. No surgical-related morality was observed and no drug specific toxicity was noted with gemcitabine, glaucarubinone or combined injections. To investigate the failure of growth in LM-P cells, the cells were subcutaneously implanted into the flank of 3 C57BL/6 mice and tumours measured. LM-P cells failed to grow above $100 \mathrm{~mm}^{3}$ and started to regress after 13 days after implantation (Fig. 3.3.9A). LM-P cells were subcutaneously implanted into 4 SCID mice, and growth was observed reaching $200 \mathrm{~mm}^{3}$ by day 19 (Fig. 3.3.9B). As a control, 2 SCID mice was implanted subcutaneously with established colorectal cancer cell line, HCT 116 and 1 SCID mouse with DLD-1. This indicated that LM-P cells may not be completely syngeneic to C57B1/6 mice. 
CHAPTER 3

A

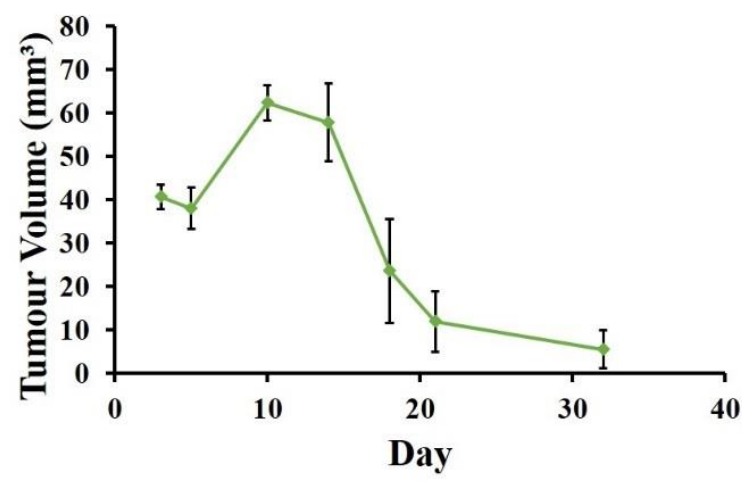

B

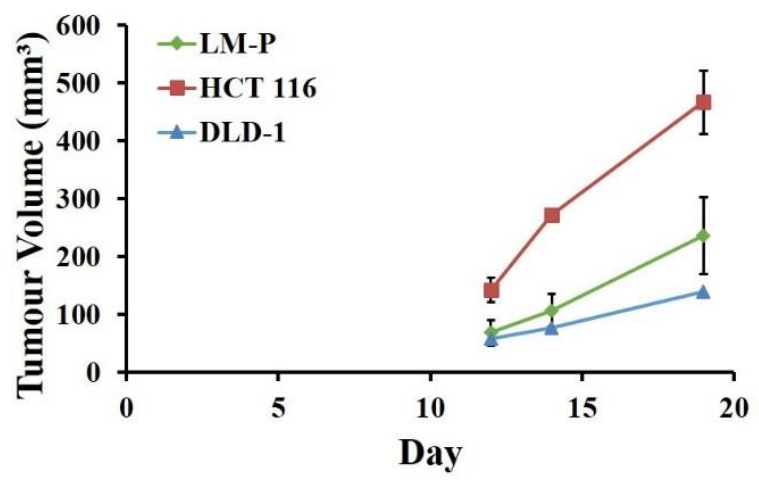

Figure 3.3.9 LM-P tumours failed to grow in C57BI/6 mice but grew in SCID mice. LM-

$\mathrm{P}$ murine pancreatic cancer cells were implanted subcutaneously into C57Bl/6 mice. Tumours started to regress after 13 days and were completely regressed after 30 days (A). LM-P cells were also implanted subcutaneously into SCID mice and growth was observed at a rate comparable to established colorectal cancer cell lines: HCT116 and DLD-1 (B). 


\section{CHAPTER 3}

\subsection{Discussion}

All six pancreatic cancer cell lines tested had PAK1 and PAK4 activity and suggests a possible role of PAK1 and PAK4 in pancreatic carcinogenesis. Among the six pancreatic cell lines tested, BxPC-3 and MiaPaCa-2 was the most sensitive, and Pan02 and LM-P the least sensitive, to glaucarubinone (Table 3.3.1). It may be more than coincidental that BxPC-3 and MiaPaCa2 cells had the lowest expression of active PAK1 and Pan02 and LM-P cells the highest (Fig. 3.3.3C). Thus, the results suggest that the anti-proliferative effect of glaucarubinone acts on PAK1, based on the $\mathrm{IC}_{50}$.

The activity of glaucarubinone has been established to involve PAK1 pathways but there is limited to no data on its involvement of PAK4. Our studies found that treatment with glaucarubinone reduced the amounts of both active PAK1 and PAK4 in human pancreatic cancer cells (Fig. 3.3.5) indicating that glaucarubinone reduced pancreatic cancer cell proliferation (Fig. 3.3.1), migration and invasion (Fig. 3.3.2) at least partly through inhibition of pathways involving PAK1 and PAK4. The mechanisms by which glaucarubinone regulates the amounts of active PAK1 has been well studied but the mechanisms involving PAK4 is limited and clearly warranted. Although gemcitabine itself did not affect the amounts of either active PAK1 or PAK4, when combined with glaucarubinone, it resulted in significantly greater reduction of the amounts of active PAK1 and PAK4 than that observed in the glaucarubinone alone treatment (Fig. 3.3.5). This reduction likely contributes to the synergistic inhibition of pancreatic cancer cell growth that results from combined treatment with gemcitabine and glaucarubinone.

The synergistic reduction in xenograft flank pancreatic cancer growth is due to the inhibition of pathways involving PAK1 and PAK4. The in vivo xenograft model showed that glaucarubinone alone and gemcitabine alone inhibited the tumour growth of MiaPaCa-2, but not PANC-1 cells. Furthermore, the combination of glaucarubinone with gemcitabine further 


\section{CHAPTER 3}

decreased the tumour growth of MiaPaCa-2 cells, and suppressed the tumour growth of PANC1 cells as well (Fig. 3.3.6). This reduction in tumour growth was mediated by the decrease in proliferation where Ki67 staining was decreased in the tumours of mice treated with glaucarbuinone and gemcitabine. Although the positive stain is high $(>60 \%)$, other methods should be examined to confirm the results such as BrdU injection prior to culling the mice or other proliferative markers such as PCNA or MCM-2. As in the in vitro study, glaucarubinone reduced the activity of PAK1 in both MiaPaCa-2 and PANC-1 tumours and the addition of gemcitabine further enhanced the reduction in PAK1 activity (Fig. 3.3.7). Although glaucarubinone alone did not significantly affect PAK4 activity, the combination of glaucarubinone with gemcitabine decreased the amount of active PAK4 in both MiaPaCa-2 and PANC-1 tumours. Although PAK4 has been shown to be a predictive marker for gemcitabine sensitivity, which may help explain the result observed, the exact mechanism which PAK4 is reduced only in the combination treatment of glaucarubinone and gemcitabine is unclear (158). These data demonstrate the clinical advantage of combining glaucarubinone to gemcitabine.

As pancreatic tumours are highly hypoxic and exhibit a high stroma content, it was important to use a clinically relevant orthotopic murine model to test the effects of glaucarubinone. As HIF-1 $\alpha$ expression was found to be regulated by PAK1-dependent pathways in glaucarubinone treated colorectal cancer cells (94) and increased HIF-1 $\alpha$ expression has been found in the pancreatic tumour stroma (99), it is highly likely that PAK1 may have an important role in both epithelial and stromal components of pancreatic cancer. Characterisation of the orthotopic murine pancreatic cancer model used has been published by our group (166). The tumours develop a dense stroma and grow and metastasize like the human pancreatic cancer. Tumours implanted within the pancreatic head cause bile duct obstruction and duodenal obstruction and produce liver and peritoneal metastases. The fact that tumours develop in a relatively short 


\section{CHAPTER 3}

period of time, compared to de novo genetically engineered models, minimises the need for extended periods of drug administration. This model was utilised to assess the effects of glaucarubinone and gemcitabine on survival, as this model is more representative of the clinical situation than the subcutaneous model that was examined in Chapter 3.3.6.

Glaucarubinone alone had no significant impact on survival but in combination with gemcitabine, significantly improved overall survival by approximately 2 -fold compared to gemcitabine alone, without increased toxicity (Fig. 3.3.8). The mechanisms for the synergistic effects of gemcitabine and glaucarubinone is uncertain. One possibility is that glaucarubinone may lead to alterations in the pancreatic stroma that improve the delivery of chemotherapy. Alternatively, glaucarubinone, by inhibition of PAK, may lead to alterations in the tumour cells that increase sensitivity to chemotherapy. For example, a recent study found that PAK4 knockdown increased the expression of the human equilibrative nucleoside transporter 1 (hENT1), which is responsible for cellular gemcitabine uptake (158). This sensitisation may not be gemcitabine chemotherapy specific and glaucarubinone treatment and more specifically, PAK inhibition, may result in sensitisation of chemotherapy as suggested by a study where glaucaurbinone sensitised oral squamous cell carcinoma to the chemotherapy, paclitaxel (107). As such, it may be plausible that glaucarubinone may have sensitised cancer cells to gemcitabine through increased expression of hENT1, particularly given that PAK4 is reduced when treated with glacuarubinone and gemcitabine in vitro (Chapter 3.3.5) and in vivo (Chapter 3.3.7).

Although both murine pancreatic cancer cell lines tested showed inhibition by glaucarubinone and gemcitabine treatment in vitro, the two cell lines differ in terms of genetic background, histological classification and derivation method and may explain the failure of LM-P cells to grow in C57B1/6 mice. Pan02 cells have a SMAD4 mutation and a single nucleotide polymorphism (SNP) in the KRAS gene, whereas LM-P cells have KRAS and TP53 mutations 
$(209,228)$. Pan02 cells was derived from pancreatic tumours induced by implanting 3 -methylcholanthrene (3-MCA)-saturated threads of cotton in the pancreas of C57BL/6 mice and classified as pancreatic cancer cells based on histological analysis (35). However, Pan02 cells lack strong clinical significance when compared to human pancreatic cancer with absence of the mutational spectrum and ductal characteristics (208). As such, LM-P cells were obtained and were planned for use. LM-P cells were derived from liver metastases from the genetically engineered mice pancreatic cancer model: $\mathrm{Kras}^{\mathrm{G} 12 \mathrm{D}} ; \operatorname{Trp} 53^{\mathrm{R} 172 \mathrm{H}} ; \mathrm{Pdx} 1-\mathrm{Cre}(\mathrm{KPC})$ mice (209). Spontaneous pancreatic tumours, primary and metastatic, result in these mice and recapitulate the clinical, histopathological and genomic features of human pancreatic cancer. The failure of LM-P cells to grow as tumours in $\mathrm{C} 57 \mathrm{Bl} / 6$ mice (Fig. 3.3.9A) is most likely due to an immune mediated phenomenon, given that discrete tumours developed in SCID mice (Fig. 3.3.9B). The original KPC mice have a mixed 129/SvJae x C57Bl/6 background and a previous orthotopic model which reported consistent growth of the LM-P cells, implanted them in B6/129 mice (209). This suggests that the cells most likely possess histocompatibility antigens, derived from their 129/SvJae background, resulting in a histocompatibility mismatch when implanted into mice from a pure $\mathrm{C} 57 \mathrm{Bl} / 6$ background. 


\subsection{Conclusion}

The two isoform of PAK, PAK1 and PAK4, that had been implicated in pancreatic cancer were confirmed and found to be expressed in all six pancreatic cancer cell lines tested. Treatment with either glaucarubinone or gemcitabine reduced pancreatic cancer proliferation, migration/invasion and the combination of the two agents was found to be synergistic in vitro. The inhibition by glaucarubinone was found, in part, acting on pathways involving PAK1 and PAK4 with further reduction observed when combined with gemcitabine although gemcitabine alone did not inhibit PAK1 or PAK4. The combination of glaucarubinone and gemcitabine treatment significantly reduced pancreatic tumour growth and improved murine survival compared to gemcitabine treatment alone, in vivo, with no apparent increase in toxicity. Although the mechanism of synergy is unclear, the results indicate the advantage of targeting PAKs in pancreatic cancer and how PAK inhibitor combination therapy can improve the outcomes of pancreatic cancer patients. 


\section{Chapter 4: FRAX597, a PAK1 Inhibitor,}

Synergistically Reduces Pancreatic Cancer Growth when Combined with Gemcitabine 


\section{CHAPTER 4}

\subsection{Introduction}

Following the findings from the previous chapter, PAK1 was investigated further using more direct mechanisms. PAK1 shRNA knockdown (KD) cells and a group 1 PAK inhibitor (with greatest inhibitory function to PAK1), FRAX597, were utilised to investigate the effect of reducing PAK1, elucidating PAK1-mediated pathways, and the clinical benefit of targeting PAK1.

FRAX597 (Figure 4.1) is a small-molecule pyridopyrimidinone that targets group 1 PAKs through binding to the ATP-binding site. Licciulli and colleagues discovered and characterised FRAX597 (123). It was designed through a traditional structure-activity relationship approach from the chemical series identified from a high throughput screen of 12000 member kinasefocused library against PAK1. Although it preferentially targets group 1 PAKs, FRAX597 has been found to also inhibit other kinases such as RET, YES1, TEK, and CSF1R (123). Of the group 1 PAKs, FRAX597 selectively inhibits PAK1 with a kinase $\mathrm{IC}_{50}$ of $8 \mathrm{nM}$, compared to $13 \mathrm{nM}$ and $19 \mathrm{nM}$ with PAK2 and PAK3, respectively with no observable inhibition of the group 2 PAKs (30). The group also found that FRAX597 inhibits proliferation in neurofibromatosis type 2 (NF2)-associated schwannomas in vitro and in vivo (123).

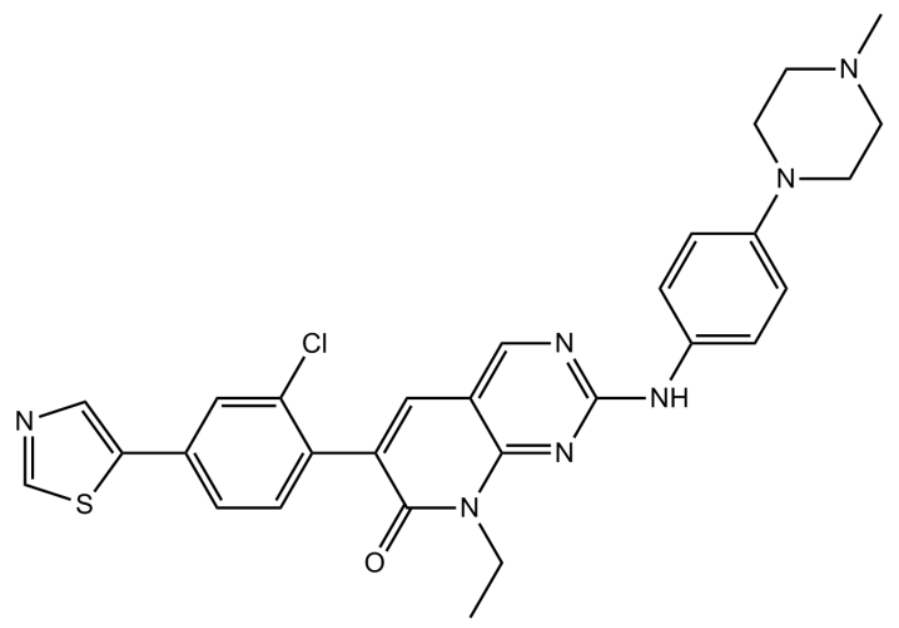

Figure 4.1 Chemical structure of FRAX597. Taken from (123) 


\section{CHAPTER 4}

Since its discovery, FRAX597 has been tested on a number of cancers to investigate PAK1 signalling. FRAX597 was used to examine the effects of PAK1 on RAS-driven skin cancer (30). The study concluded that PAK1 was signalling through the ERK pathway in KRASdriven squamous cell carcinomas as tumour regression was observed when FRAX597 and a MEK inhibitor but not an AKT inhibitor was administered in vivo. FRAX597 was tested to investigate PAK1 as a therapeutic target in acute myeloid leukemia and myelodysplastic syndrome (172). Treatment with FRAX597 resulted in reduced growth, induction of apoptosis, reduced leukemic colony formation, and reduced leukemia stem cell-enriched populations in vitro. Finally, FRAX597 has been used to investigate PAK1 signalling as a downstream effector of KRAS in the Crk pathway in non-small cell lung cancer (160). The combination of KRAS prenylation inhibitors with FRAX597 was used to show that disruption of KRAS and PAK1 pathways resulted in altered cell morphology, reduced motility and reduced proliferation in vitro.

FRAX597 has also been used to investigate pancreatic acinar cells in response to gastrointestinal hormones and neurotransmitters (167). However, the study used FRAX597 as an inhibitor of PAK2 rather than PAK1 as the authors found that only PAK2 was present in pancreatic acinar cells and was required for enzyme-secretion and ERK1/2 activation. The study concluded that PAK2 is important in mediating cholecystokinin's effect on the activation of signalling pathways in response to the onset of pancreatitis. FRAX597, as a group 1 PAK inhibitor, has been examined in a number of cancers and in the acinar cells of the pancreas, but has not been examined in pancreatic cancer. This study investigated the role of PAK1 in pancreatic cancer by examining the effects of reducing PAK1 activity with the selective inhibitor, FRAX597, or PAK1 expression by shRNA KD in vitro and in vivo, alone and in combination with gemcitabine. 


\section{CHAPTER 4}

\subsection{Methods}

The proliferation, survival, toxicity, combination index, sphere formation, migration and invasion, hypoxia treatment of cells, and Western blot assays; orthotopic pancreatic cancer murine implantation, tissue processing, immunohistochemical staining procedures, and picrosirius red staining have been described in Chapter 2 .

\subsection{1 shRNA Transfection}

PAK1 knockdown (KD) clones were obtained from PANC-1 and MiaPaCa-2 cells as described (Chapter 2.3). For PANC-1, $2 \mathrm{mg} / \mathrm{ml}$ of G418 was added, while for MiaPaCa-2, $1 \mathrm{mg} / \mathrm{ml}$ of G418 was added to the cells the following day after transfection.

\subsubsection{Orthotopic Pancreatic Cancer Murine Study Design}

Pan02 (2.5 x $10^{5}$ cells) were suspended in $50 \mu \mathrm{L}$ of Matrigel $^{\mathrm{TM}}$ matrix solution (BD Biosciences) and implanted into the pancreatic tail or pancreatic head of $\mathrm{C} 57 \mathrm{Bl} / 6$ mice as described in Chapter 2.13. Leakage occurred in less than $5 \%$ of mice, and only mice without leakage during injection were included in experiments. Mice were monitored based on health score for up to 30 days for the pancreatic tail model or for up to 45 days for the pancreatic head model and euthanised when a poor health score was reached.

In the pancreatic tail model, assessment of tumour growth and metastasis was undertaken where 28 mice were implanted with Pan02 cells and groups of 7 mice were randomly allocated to one of four groups:

1. Control: i.p. saline injection every other day;

2. FRAX597 alone: glaucarubinone (3mg/kg) i.p. every other day;

3. Gemcitabine alone: gemcitabine $(40 \mathrm{mg} / \mathrm{kg})$ i.p. twice weekly; or 


\section{CHAPTER 4}

4. Combination of FRAX597 and gemcitabine: following the treatment as described above.

Treatment started 7 days after tumour implantation, at a time when discrete tumours had formed. At the endpoint, tumours were isolated, measured (as described in Chapter 2.13), extracted, and harvested for histological analysis as described in Chapter 2.14.

In the pancreatic head model, assessment of murine survival was undertaken over two studies. In the first study, 24 mice were implanted with Pan02 cells and groups of 12 mice were randomly allocated to either control or gemcitabine treatment alone as described above. In the second study, 30 mice were implanted with Pan02 cells, with 12 mice randomly allocated to either gemcitabine alone or combination treatment and remaining 4 mice allocated to the control group as described above. Treatment started 7 days after tumour implantation, at a time when discrete tumours had formed. A collated Kaplan-Meier survival curve was plotted (SPSS). At the endpoint, tumours were isolated, extracted, and harvested for histological analysis as described in Chapter 2.14 . 
CHAPTER 4

\subsection{Results}

\subsubsection{PAK1 is upregulated in human pancreatic cancer and pancreatic cancer cell lines}

PAK1 staining was observed in pancreatic ductal adenocarcinoma cells in all 20 human pancreatic cancer samples tested (Fig. 4.3.1A). Corresponding normal pancreas samples had no PAK1 staining in acinar and ductal epithelial cells but islet cells did stain for PAK1. Low levels of PAK1, and of the active form of PAK1 (pPAK1) protein expression was detected in the normal human pancreatic duct epithelial (HPDE) cells and was significantly lower compared to all the pancreatic cancer cell lines tested (Fig. 4.3.1B). 
A

Normal Pancreas
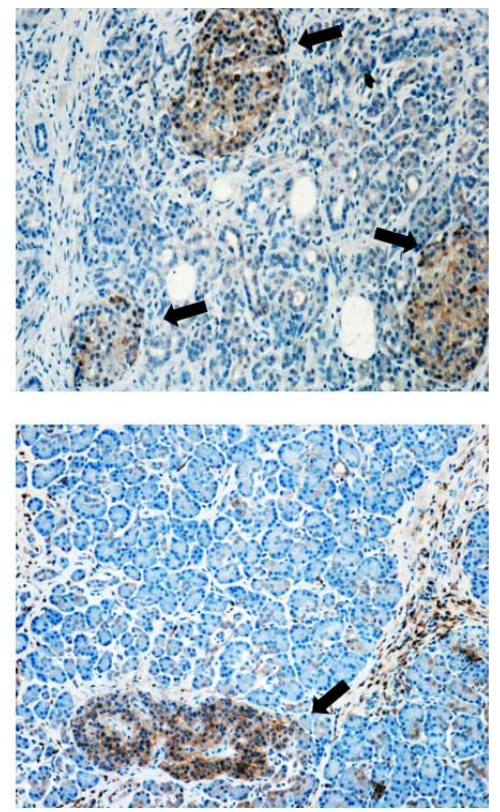

Pancreatic Cancer
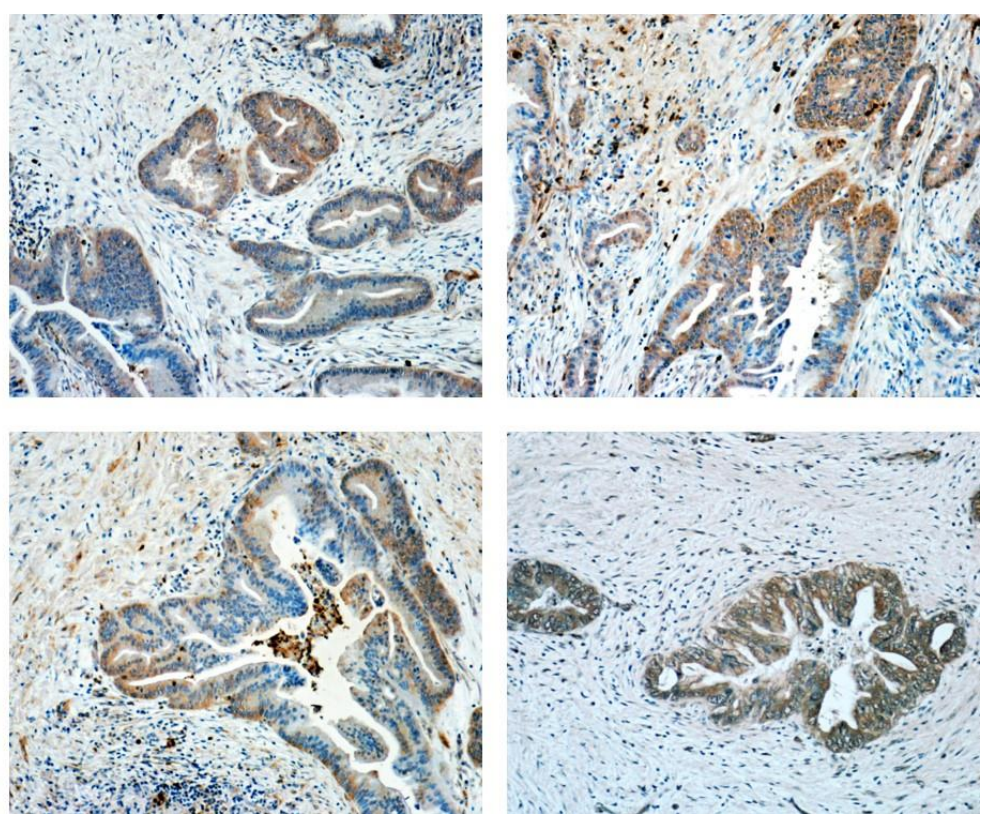

B

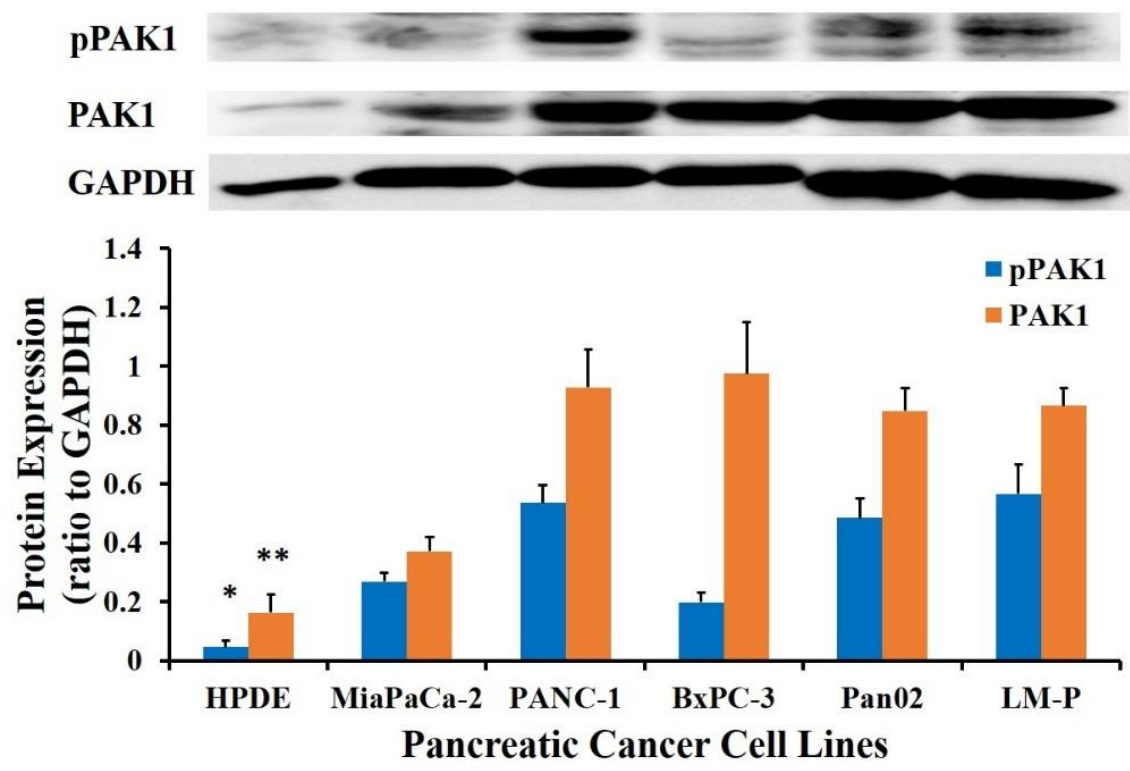

Figure 4.3.1 PAK1 is upregulated in pancreatic cancer specimens and pancreatic cancer cell lines. Acinar and ductal cells in normal pancreas are not stained for PAK1 by immunohistochemistry (IHC), but islet cells (denoted by the arrow) are positive (A). Magnification: 200x. Pancreatic ductal adenocarcinoma cells in pancreatic cancer samples stain positive for PAK1. Magnification: 100x. The normal human pancreatic duct epithelial 


\section{CHAPTER 4}

cell line, HPDE, expressed low levels of active PAK1 (pPAK1) and PAK1 as detected by Western blotting. All pancreatic cancer cell lines, MiaPaCa-2, PANC-1, BxPC-3, Pan02 and LM-P expressed pPAK1 and PAK1. The data represent mean \pm SEM, summarised from three independent experiments. ${ }^{*} \mathrm{p}<0.05,{ }^{* *} \mathrm{p}<0.01$ compared to all other pancreatic cancer cell lines.

\subsubsection{Inhibition of PAK1 by shRNA knockdown decreased proliferation and survival of pancreatic cancer cells}

The PAK1 protein levels in two PANC-1 PAK1 knockdown (KD) clones (2.05 and 2.10), transfected with shRNA, were decreased to $22 \%$ and $24 \%$, respectively, compared to the corresponding NC cells, which had been transfected with scrambled sequences (Fig. 4.3.2A). Similarly, the PAK1 protein levels in two MiaPaCa-2 PAK1 KD clones (3.09 and 3.12) were decreased to $11 \%$ and $9 \%$, respectively, of the PAK1 protein expression of the corresponding NC cells (Fig. 4.3.2B). As a transfection control, PAK2 expression was checked, as PAK2 has similar sequence homology compared to PAK1. The PAK2 expression of the PAK1 KD clones were not significantly different compared to the NC cells (Fig. 4.3.2C-D). This result indicates that the knockdown of PAK1 by shRNA transfection was successful and specific to PAK1.

The proliferation rate was significantly reduced in both PANC-1 (Fig. 4.3.2E) and MiaPaCa-2 (Fig. 4.3.2F) PAK1 KD cells compared to the corresponding NC cells. The growth rate of two clones of PANC-1 PAK1 KD cells $\left(1.9 \% \mathrm{hr}^{-1}\right.$ and $\left.2.1 \% \mathrm{hr}^{-1}\right)$ was significantly less than the NC cells $\left(2.4 \% \mathrm{hr}^{-1}\right.$ and $\left.2.6 \% \mathrm{hr}^{-1}\right)$ (Table 4.3.2). Similarly, the growth rate of the two clones of MiaPaCa-2 PAK1 KD cells $\left(2.0 \% \mathrm{hr}^{-1}\right.$ and $\left.2.1 \% \mathrm{hr}^{-1}\right)$ was significantly less than the NC cells $\left(2.9 \% \mathrm{hr}^{-1}\right.$ and $\left.2.7 \% \mathrm{hr}^{-1}\right)$. Cell survival was significantly decreased in PANC-1 (Fig. 4.3.2G) PAK1 KD cells compared to NC cells. No difference was observed in MiaPaCa-2 (Fig. 4.3.2H) 
CHAPTER 4

PAK1 KD cells. These results suggest that PAK1 is involved in pancreatic cancer cell proliferation and may also be involved in cell survival but is cell line specific as PANC-1 and MiaPaCa-2 had different PAK1 expression initially prior to the knockdown so the cell's reliance on PAK1-dependent pathways may differ. 
A

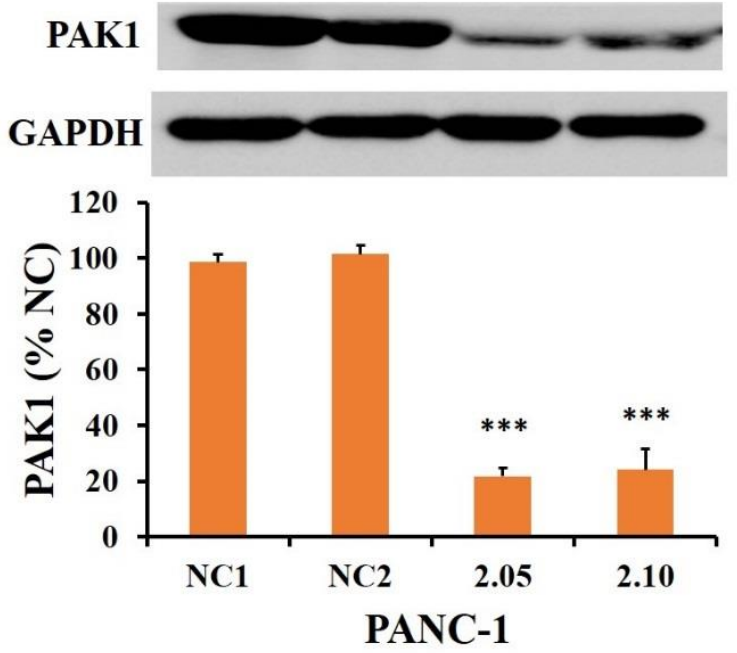

C
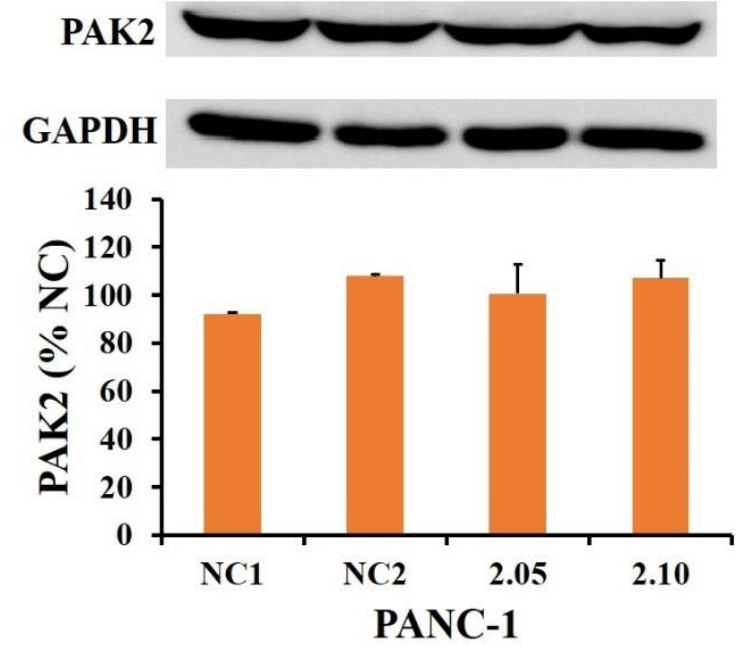

E

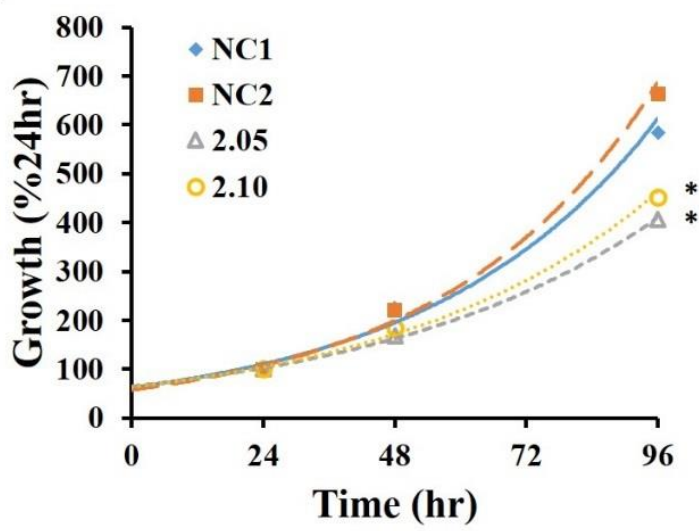

B

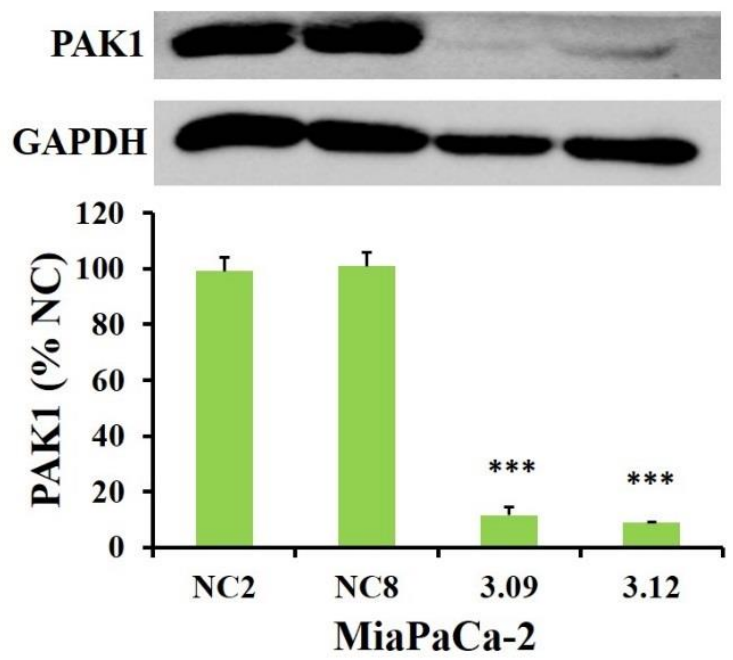

D

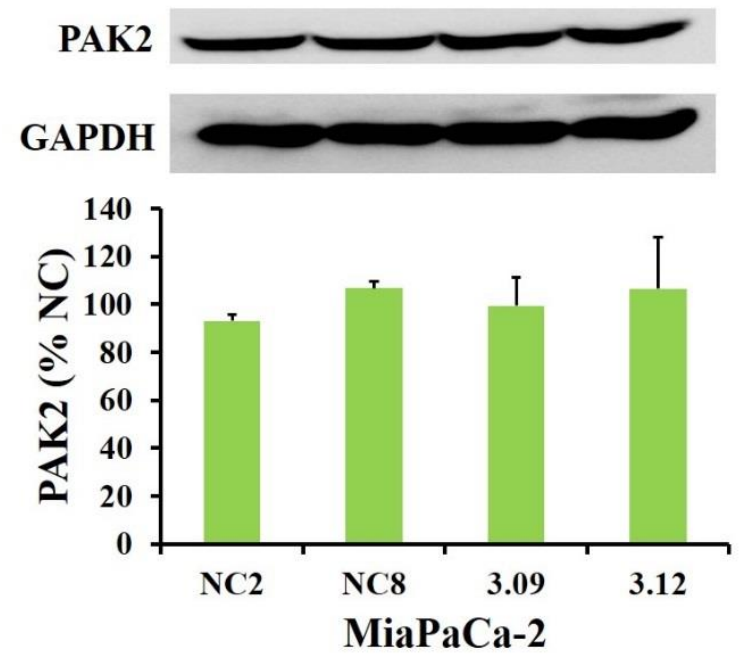

F

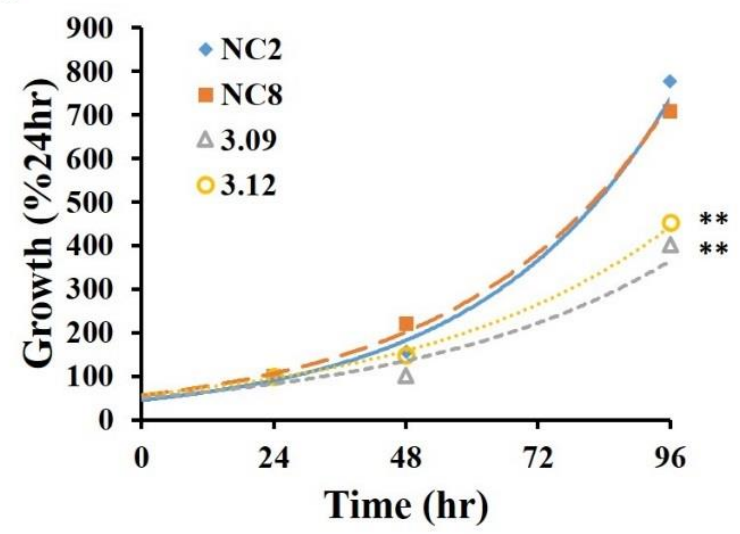




\section{CHAPTER 4}
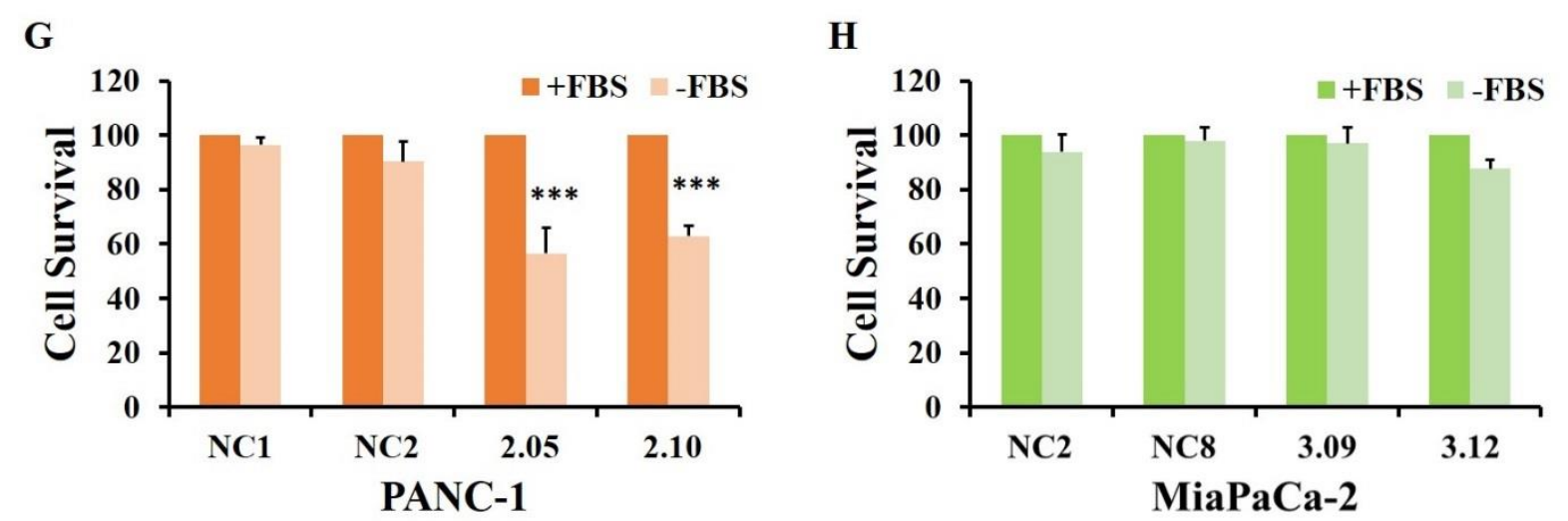

Figure 4.3.2 PAK1 shRNA knockdown (KD) was specific to PAK1 and PAK1 KD reduced cell proliferation and survival. PANC-1 (A) and MiaPaCa-2 (B) PAK1 KD cells were generated using shRNA transfection. As detected by Western blot, clones 2.05 and 2.10; and clones 3.09 and 3.12 for PANC-1 and MiaPaCa-2, respectively, expressed significantly less PAK1 than negative control (NC) clones, which had been transfected with a scrambled shRNA. There was no change in PAK2 expression in the PANC-1 (C) or MiaPaCa-2 (D) PAK1 KD clones showing specificity of the knockdown. The proliferation rate of the KD clones for both PANC-1 (E) and MiaPaCa-2 (F), measured by thymidine incorporation, was significantly lower after 96 hours. Survival of PAK1 KD cells was measured using thymidine-withdrawal as a percentage of the thymidine level in the absence (lighter bars) of serum to the thymidine level in the presence (darker bars) of serum as described in Chapter 2.14. Survival in PANC-1 PAK1 KD clones (A) were significantly lower compared to the NC cells but no difference was observed in MiaPaCa-2 PAK1 KD clones (B). ${ }^{*} \mathrm{p}<0.05,{ }^{* *} \mathrm{p}<0.01,{ }^{* * *} \mathrm{p}<0.001$ compared to either NC clone (where the higher $\mathrm{p}$ value of the two is presented). 


\section{CHAPTER 4}

Table 4.3.2: PAK1 knockdown (KD) inhibited cell growth

Growth Rate $\left(\% \mathrm{hr}^{-1}\right)$

PANC-1 MiaPaCa-2

\begin{tabular}{lcc}
\hline $\mathrm{NC} 1$ & 2.4 & 2.9 \\
$\mathrm{NC} 2$ & 2.6 & 2.7 \\
$\mathrm{KD} 1$ & $1.9 *$ & $2.0 * *$ \\
$\mathrm{KD} 2$ & $2.1 *$ & $2.1 * *$ \\
\hline
\end{tabular}

$\mathrm{NC} 1$ and $\mathrm{NC} 2$ indicate PANC-1 NC clones NC1 and NC2; and MiaPaCa-2 NC clones NC2 and NC8 respectively. KD1 and KD2 indicate PANC-1 KD clones 2.05 and 2.10; and MiaPaCa-2 KD clones 3.09 and 3.12 respectively. ${ }^{*} \mathrm{p}<0.05,{ }^{* *} \mathrm{p}<0.01$, compared to either NC clone (where the higher $\mathrm{p}$ value of the two was used).

\subsubsection{Inhibition of PAK1 by shRNA knockdown sensitised pancreatic cancer cells to} gemcitabine

Proliferation of PANC-1 (Fig. 4.3.3A) and MiaPaCa-2 (Fig. 4.3.3B) PAK1 KD cells treated with gemcitabine was inhibited to a greater extent than the corresponding $\mathrm{NC}$ cells at concentrations of $20 \mathrm{nM}$ and $50 \mathrm{nM}$. The $\mathrm{IC}_{50}$ values of two clones of PANC-1 PAK1 KD cells (20nM and 21nM) (Table 4.3.3), were significantly less than the values for NC cells (26nM and $39 \mathrm{nM}$ ). Similarly, the $\mathrm{IC}_{50}$ values of two clones of MiaPaCa-2 PAK1 KD cells (26nM and $25 \mathrm{nM})$ (Table 4.3.3) were significantly less than the values for $\mathrm{NC}$ cells $(29 \mathrm{nM}$ and $28 \mathrm{nM})$. These results indicate that PAK1 inhibition increased sensitivity of gemcitabine on pancreatic cancer proliferation. 
A

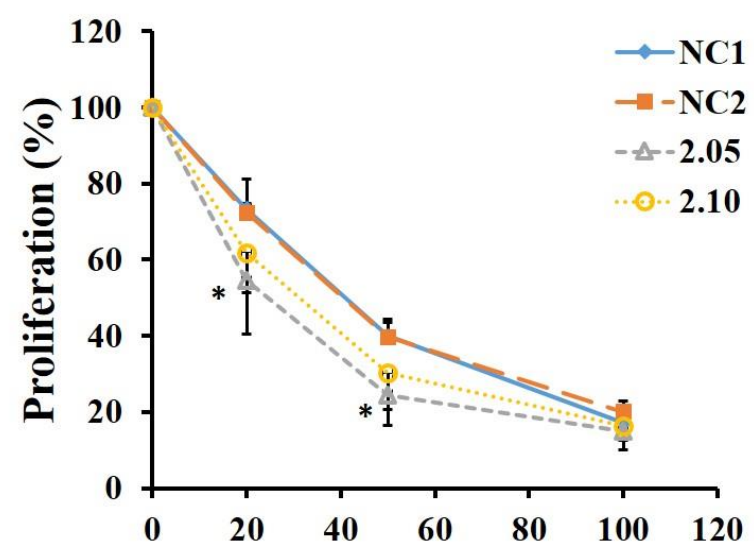

Gemcitabine (nM)
B

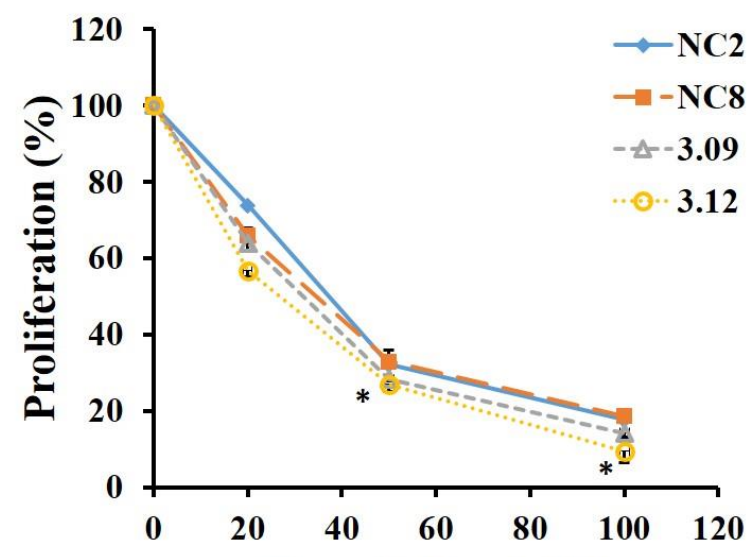

Gemcitabine (nM)

Figure 4.3.3 PAK1 knockdown (KD) increased gemcitabine sensitivity. Sensitivity of the PAK1 KD clones to gemcitabine was significantly increased with significant reduction in proliferation observed at $20 \mathrm{nM}$ and $50 \mathrm{nM}$ for PANC-1 (A) and at 50nM and 100nM for MiaPaCa-2 (B). The data represent mean \pm SEM, summarised from three independent experiments. ${ }^{*} \mathrm{p}<0.05$ compared to either $\mathrm{NC}$ clone (only the higher $\mathrm{p}$ value of the two is presented). 


\section{CHAPTER 4}

Table 4.3.3: PAK1 knockdown (KD) decreased gemcitabine $\mathrm{IC}_{50}$.

\begin{tabular}{lcc} 
& \multicolumn{2}{c}{ Gemcitabine $\mathrm{IC}_{50}(\mathrm{nM})$} \\
& PANC-1 & MiaPaCa-2 \\
& $26 \pm 2$ & $29 \pm 1$ \\
$\mathrm{NC1}$ & $39 \pm 1$ & $28 \pm 1$ \\
$\mathrm{NC} 2$ & & \\
$\mathrm{KD} 1$ & $20 \pm 2 * *$ & $26 \pm 1 *$ \\
$\mathrm{KD} 2$ & $21 \pm 2 *$ & \\
\hline
\end{tabular}

$\mathrm{NC} 1$ and $\mathrm{NC} 2$ indicate PANC-1 NC clones NC1 and NC2; and MiaPaCa-2 NC clones NC2 and $\mathrm{NC} 8$ respectively. KD1 and $\mathrm{KD} 2$ indicate PANC-1 KD clones 2.05 and 2.10; and MiaPaCa-2 KD clones 3.09 and 3.12 respectively. ${ }^{*} \mathrm{p}<0.05 ;{ }^{* *} \mathrm{p}<0.01$, compared to either NC clone (where only the higher $\mathrm{p}$ value of the two was used).

\subsubsection{Inhibition of PAK1 by shRNA knockdown decreased clonogenic growth}

Tumour sphere formation ability was measured in PANC-1 parent, NC and PAK1 KD cells. PANC-1 PAK1 KD cells had significantly reduced number of colonies compared to the parent and NC cells at all cell seeding numbers tested (Fig. 4.3.4). A significant decrease in tumour colonies for NC cells compared to the parent cells was also observed which may be in part due to the transfection and selection process. The sphere formation assay is an indirect measure of tumourigenicity, so the results suggest that reducing PAK1 decreases tumourigenicity. 


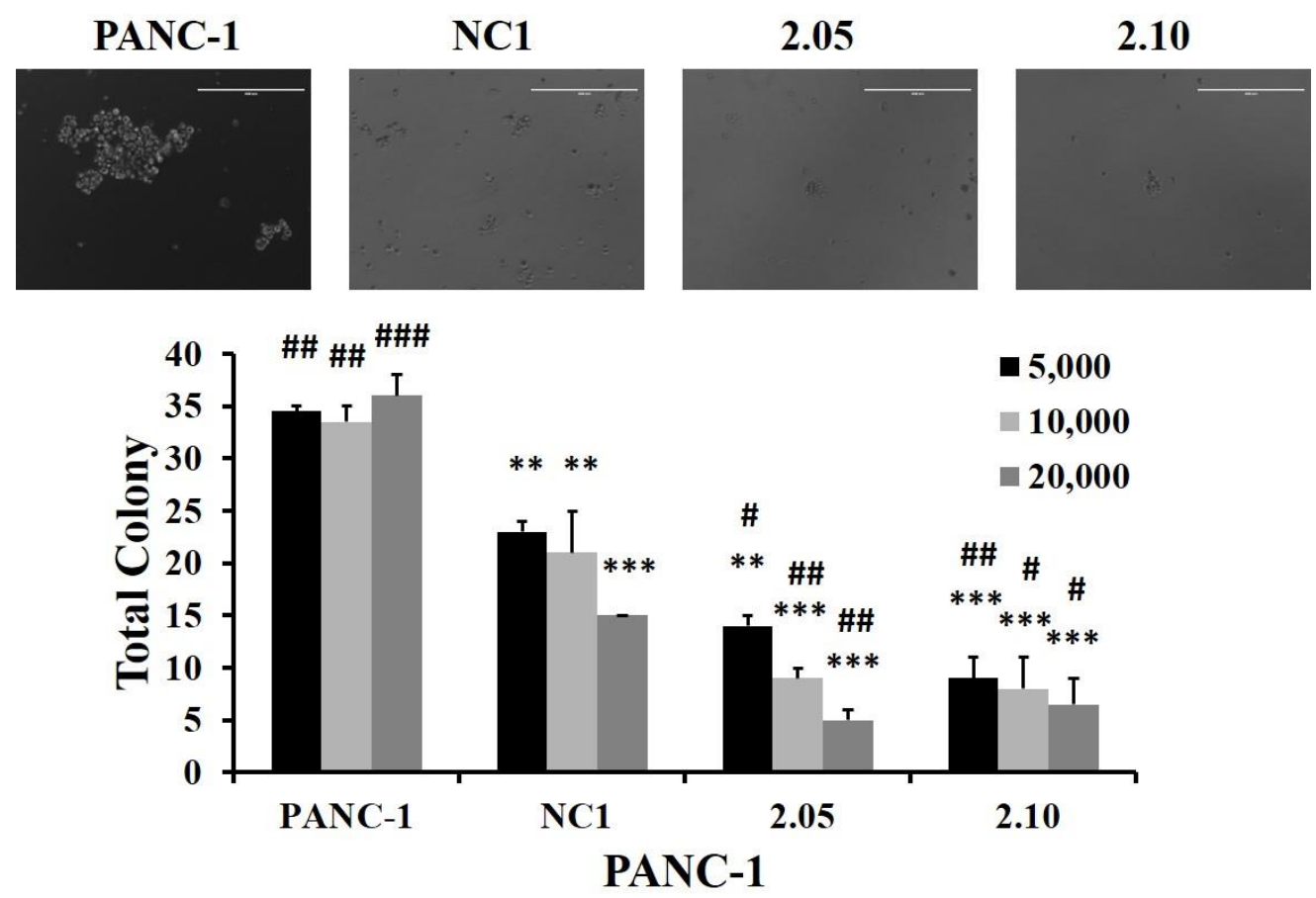

Figure 4.3.4 PAK1 knockdown (KD) cells had decreased sphere formation. Tumourigenicity was measured in PANC-1 parent, NC and PAK1 KD cells at a seeding number of 5000, 10000, and 20000. Total number of colonies were counted for each well after 10 days. NC cells had significantly decreased number of colonies compared to the parent PANC-1 cells at all seeding numbers. Both PAK1 KD cells had significantly reduced number of colonies compared to the NC cells indicating reduced tumourigenicity. The data represent mean $\pm \mathrm{SEM}$, summarised from three independent experiments. ${ }^{*} \mathrm{p}<0.05 ;{ }^{* *} \mathrm{p}<0.01, * * *$ $\mathrm{p}<0.001$ compared to PANC-1 parent; \# $\mathrm{p}<0.05$; \#\# $\mathrm{p}<0.01$, \#\#\# $\mathrm{p}<0.001$ compared to NC1.

4.3.5 Inhibition of PAK1 by shRNA knockdown reduced AKT activity and HIF-1 $\alpha$ expression

PAK1-mediated signalling pathways have been well studied in other cancers. PAK1 has been known to mediate cross-talk between the PI3K pathway (AKT) and the MAPK pathway (ERK) in colorectal cancer as well as survival under hypoxic conditions (HIF1 $\alpha)$. PAK1 has been 
linked to $\beta$-catenin and VEGF activation in colorectal and breast cancer growth and transformation. PAK1 is also involved in migration and EMT so markers: MMP9 and Vimentin was also assessed.

AKT activity was significantly reduced in both PANC-1 PAK1 KD cells, by $22 \%$ and $31 \%$ (Fig. 4.3.5A), and in both MiaPaCa-2 PAK1 KD cells by $24 \%$ and 33\% (Fig. 4.3.5B). HIF1 $\alpha$ expression was significantly reduced in both clones of PANC-1 (Fig. 4.3.5C) and MiaPaCa-2 (Fig. 4.3.5D) PAK1 KD cells compared to the NC cells under normoxia and hypoxia. ERK activity was not significantly changed in either PANC-1 (Fig. 4.3.5E) or MiaPaCA-2 (Fig. 4.3.5F) PAK1 KD cells compared to the NC cells. Both PANC-1 and MiaPaCa-2 PAK1 KD cells had reduced VEGF expression compared to NC (Fig. 4.3.5G-H). Cell line specific protein changes were observed with PANC-1 PAK1 KD cells having reduced MMP9 and $\beta$-catenin expression (Fig. 4.3.5G) and MiaPaCa-2 PAK1 KD cells with reduced vimentin expression (Fig. 4.3.5H). 
A

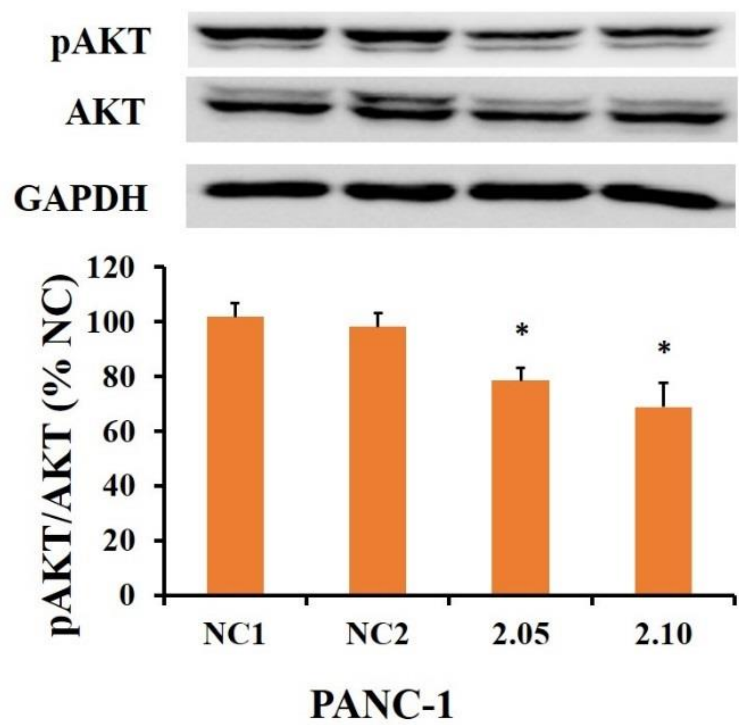

C
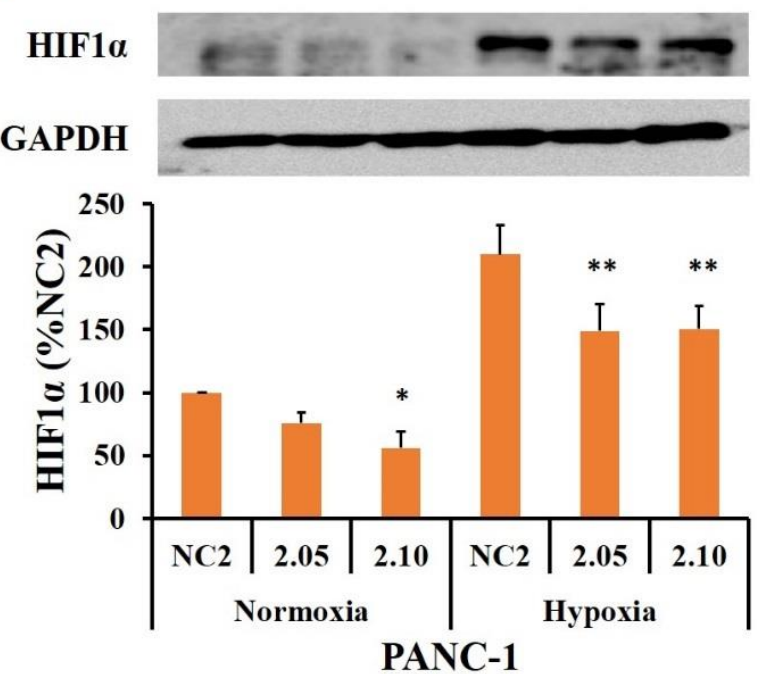

B
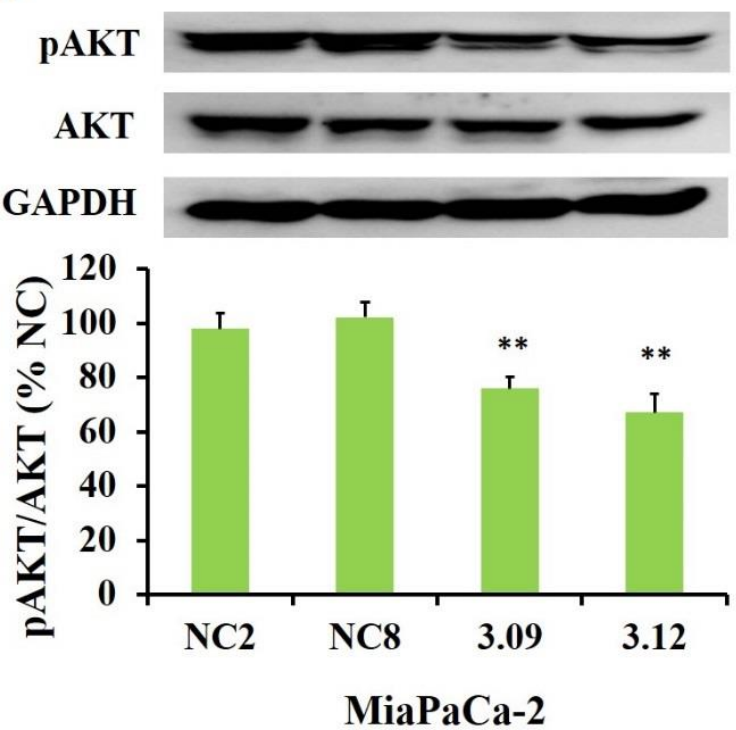

D
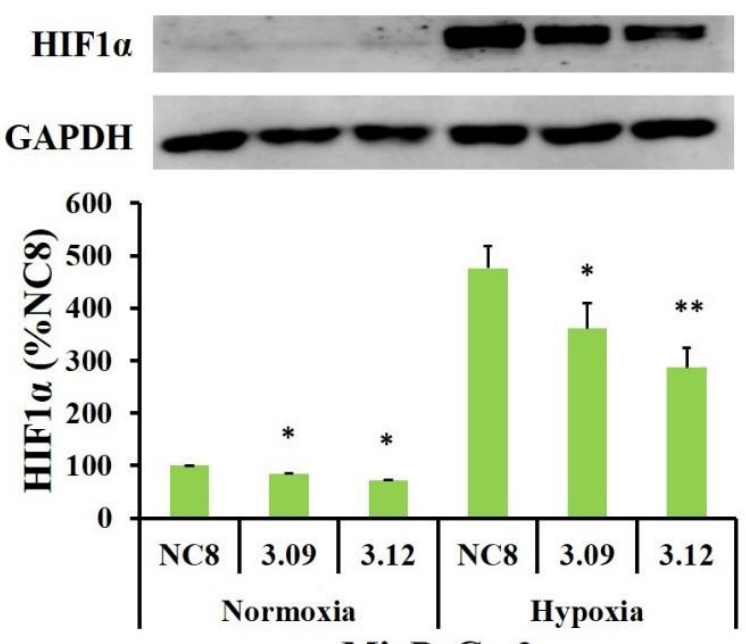

MiaPaCa-2 

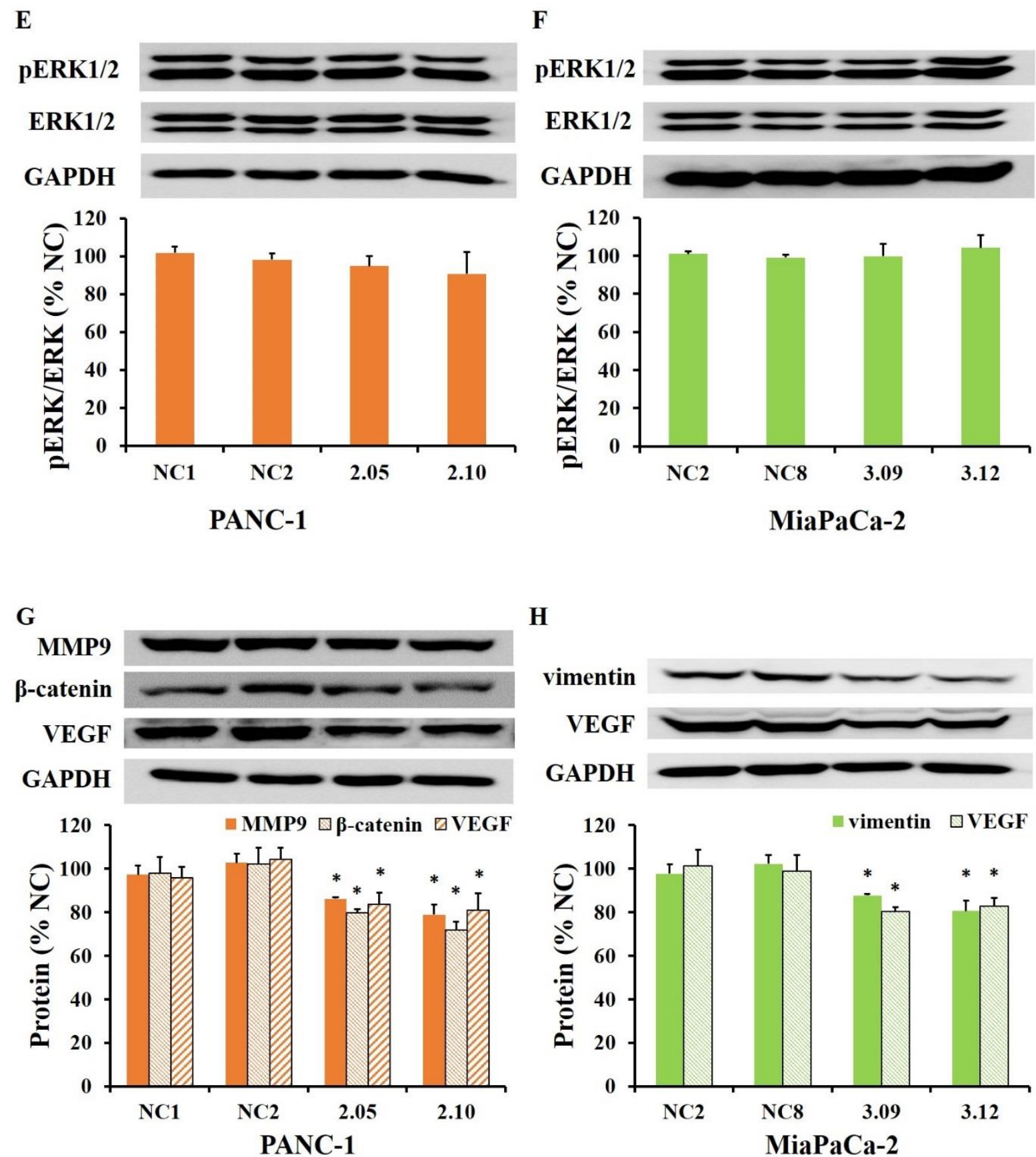

Figure 4.3.5 PAK1 knockdown (KD) inhibited AKT activity and HIF1a expression. Expression of active form of AKT (pAKT) was significantly reduced in the PAK1 KD clones: 2.05 and 2.10 (PANC-1 (A)); and 3.09 and 3.12 (MiaPaCa-2 (B)), compared to the negative controls (NC), as assessed by Western blot. HIF1 $\alpha$ expression was reduced in PANC-1 (C) and MiaPaCa-2 (D) PAK1 KD clones under normoxia and hypoxia $\left(1 \% \mathrm{O}_{2}\right)$ conditions. ERK1/2 was unchanged in PAK1 KD clones for both PANC-1 (E) and MiaPaCa-2 (F) cells. PANC-1 


\section{CHAPTER 4}

PAK1 KD cells had reduced MMP9, $\beta$-catenin and VEGF expression (G) and MiaPaCa-2 had reduced vimentin and VEGF expression $(\mathrm{H})$. The data represent mean \pm SEM, summarised from three independent experiments. ${ }^{*} \mathrm{p}<0.05,{ }^{* *} \mathrm{p}<0.01$ compared to either $\mathrm{NC}$ clone (only the higher $\mathrm{p}$ value of the two is presented).

\subsubsection{Inhibition of PAK1 by shRNA knockdown was transient in murine pancreatic cancer cell lines}

PAK1 KD cells were attempted in the two murine pancreatic cancer cell lines: Pan02 and LMP. shRNA transfection and selection were completed successfully with early passaged PAK1 KD clones having significantly decreased PAK1 expression found in clones 3.01 and 3.03 for Pan02, and clones 2.22 and 3.20 for LM-P (Fig. 4.3.6A\&C). This knockdown was not maintained as 3 passages later, the clones had similar PAK1 levels as the NC cells for both murine pancreatic cancer cell lines (Fig. 4.3.6B\&D). The transfection was repeated a number of times however, a stable knockdown could not be obtained as the PAK1 expression would increase to parent cell expression after a few passages, regardless of the initial knockdown expression. 


\section{CHAPTER 4}

A

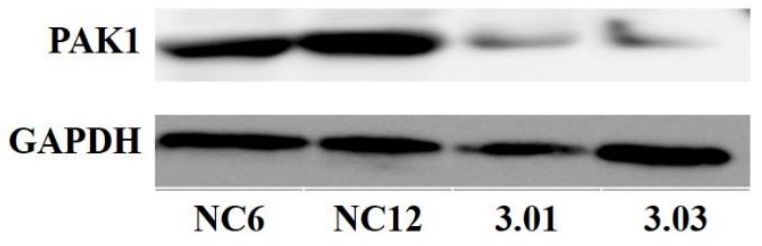

C

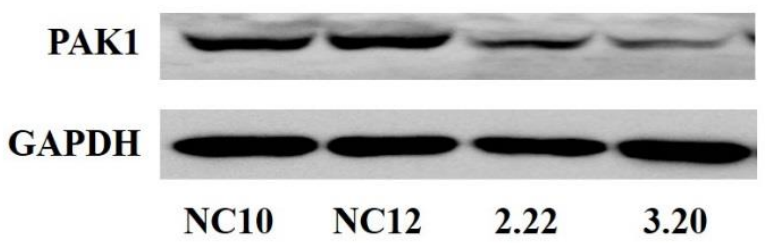

B

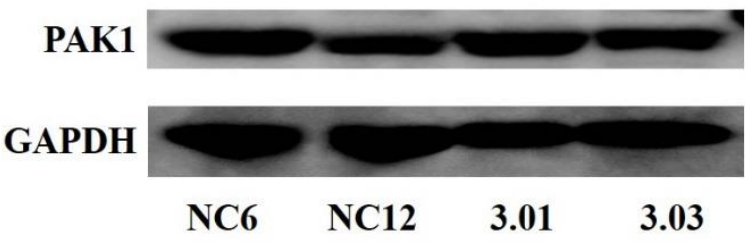

D

PAK1

GAPDH

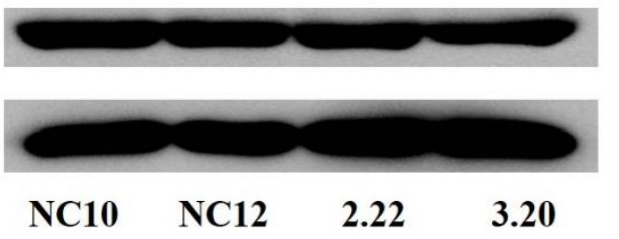

Figure 4.3.6 PAK1 knockdown (KD) of murine pancreatic cancer cells was transient. Pan02 (A-B) and LM-P (C-D) PAK1 KD cells were generated using shRNA transfection. As detected by Western blot, clones 3.01 and 3.03; and clones 2.22 and 3.20 for Pan02 (A) and LM-P (C), respectively, expressed reduced PAK1 compared to negative control (NC) clones, which had been transfected with a scrambled shRNA. However, after three passages, the PAK1 expression returned to normal levels compared to NC for both Pan02 (B) and LM-P (D) clones.

4.3.7 FRAX597 decreased proliferation and migration/invasion in pancreatic cancer cell lines

FRAX597 inhibited proliferation in all pancreatic cancer cell lines in a dose-dependent manner (Fig. 4.3.7A), with $\mathrm{IC}_{50}$ values ranging from $0.6 \mu \mathrm{M}$ for BxPC-3 cells to $2.0 \mu \mathrm{M}$ for PANC-1 cells (Table 4.3.7). Similarly, FRAX597 inhibited migration and invasion in all pancreatic cancer cell lines in a dose-dependent manner (Fig. 4.3.7B), with $\mathrm{IC}_{50}$ values ranging from 105nM for MiaPaCa-2 cells to 605nM for Pan02 cells (Table 4.3.7). FRAX597 inhibited survival of LM-P cells in a dose-dependent manner with an $\mathrm{IC}_{50}$ value of $1.10 \mu \mathrm{M}$ (Fig. 4.3.7C). Significant inhibition of survival of PANC-1, MiaPaCa-2, BxPC-3, and Pan02 cells was only observed at concentrations greater than $1 \mu \mathrm{M}$. 

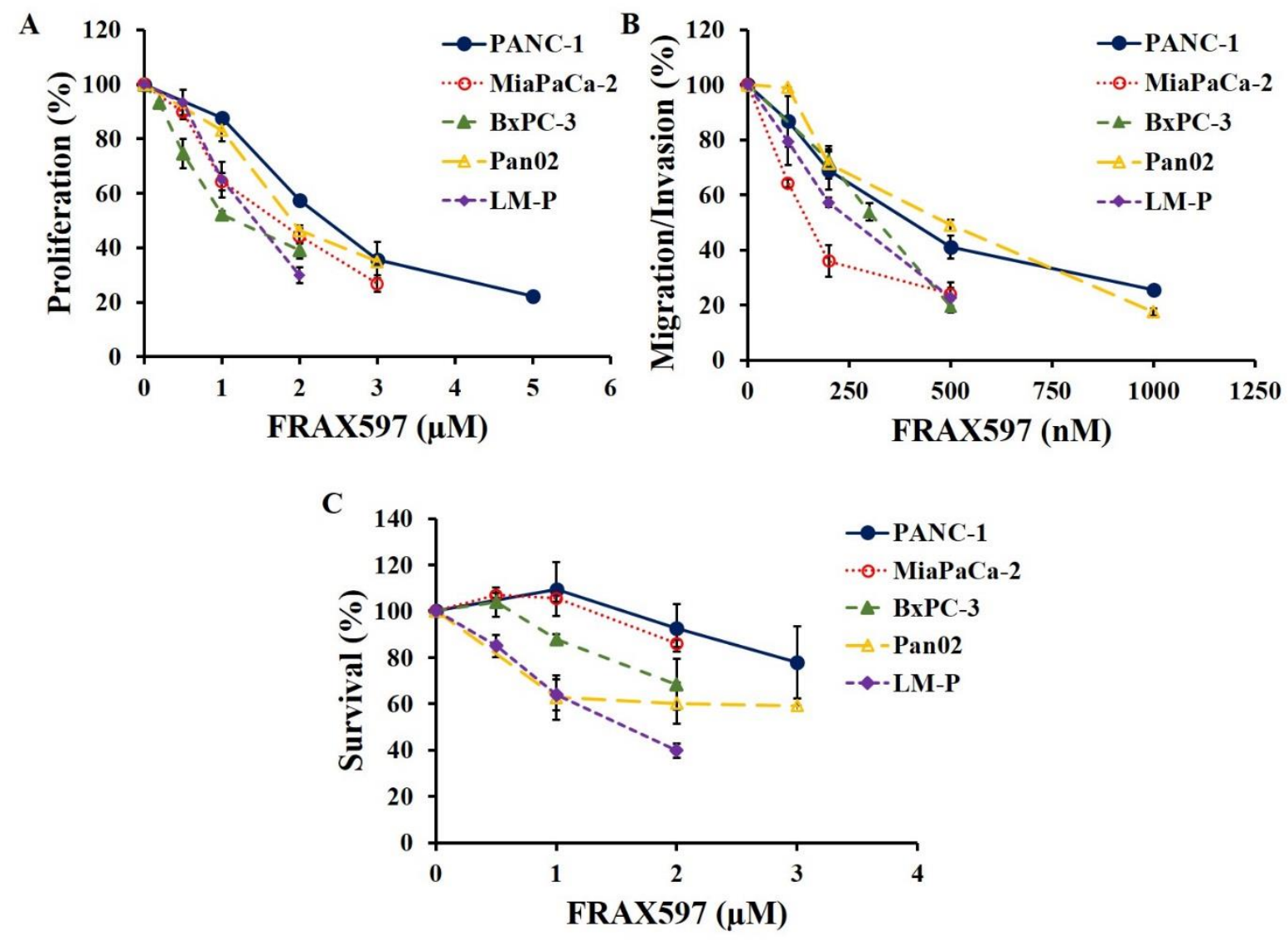

Figure 4.3.7 FRAX597 inhibited pancreatic cancer proliferation, migration/invasion and survival in a dose dependent manner. The group 1 PAK inhibitor, FRAX597, inhibited cell proliferation (A), cell migration/invasion (B) and cell survival (C) on the human pancreatic cancer cell lines: PANC-1, MiaPaCa-2, BxPC-3; and the murine pancreatic cancer cell lines: Pan02, LM-P, measured using the thymidine-incorporation method, the Transwell Boyden chamber assay, and the thymidine-withdrawal method, respectively. The values for the untreated cells were taken as $100 \%$. The data represent mean \pm SEM, summarised from three independent experiments. Significance is not shown for clarity. 


\section{CHAPTER 4}

Table 4.3.7: Proliferation and migration/invasion $\mathrm{IC}_{50}$ values for FRAX597

$$
\text { BxPC-3 MiaPaCa-2 PANC-1 LM-P Pan02 }
$$

\begin{tabular}{lccrrr}
\hline Proliferation $\mathrm{IC}_{50}(\mu \mathrm{M})$ & $0.6 \pm 0.1$ & $1.4 \pm 0.4$ & $2.0 \pm 0.2$ & $1.1 \pm 0.2$ & $1.4 \pm 0.1$ \\
Migration/Invasion $\mathrm{IC}_{50}(\mathrm{nM})$ & $330 \pm 45$ & $105 \pm 10$ & $290 \pm 70$ & $150 \pm 25$ & $605 \pm 80$
\end{tabular}

\subsubsection{FRAX597 and gemcitabine synergistically inhibited pancreatic cancer cell growth}

The combined effects of FRAX597 and gemcitabine on proliferation were measured by incubation of pancreatic cancer cells with increasing concentrations of gemcitabine, alone or in combination with FRAX597, at a concentration close to the $\mathrm{IC}_{50}$ value given in Table 4.3.7. A further reduction of proliferation was observed when treated with the combination of FRAX597 and gemcitabine, compared to gemcitabine alone in all pancreatic cancer cell lines tested (Fig. 4.3.8A-E). The combined effect of FRAX597 and gemcitabine was shown to be synergistic $(\mathrm{CI}<1)$ when analysed using the Chou-Talalay method for all pancreatic cancer cell lines tested (Fig. 4.3.8F). These results demonstrated that FRAX597 and gemcitabine synergistically inhibited pancreatic cancer cell growth. 

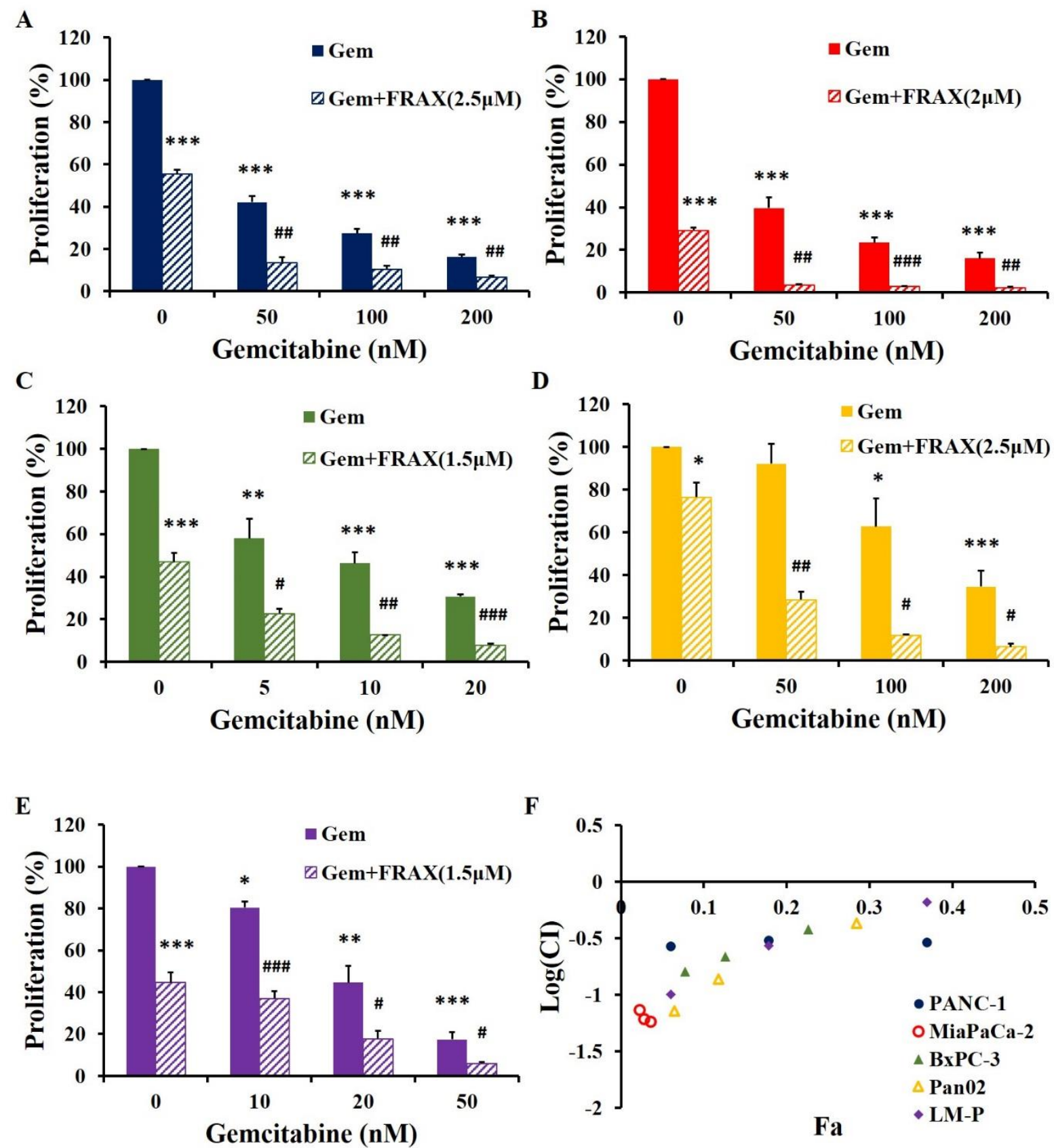

Figure 4.3.8 FRAX597 synergises with gemcitabine to inhibit pancreatic cancer proliferation. The effects of gemcitabine alone (Gem, solid bars), and gemcitabine with FRAX597 (Gem + FRAX, striped bars), on proliferation of PANC-1 (A), MiaPaCa-2 (B), BxPC-3 (C), Pan02 (D), and LM-P (E) cells were assessed by thymidine incorporation. The concentration of FRAX597 used was based on the $\mathrm{IC}_{50}$ value determined in Chapter 4.3.7. The combination index (CI), calculated by the Chou-Talalay method, was used to determine the 


\section{CHAPTER 4}

mechanism of action of FRAX597 and gemcitabine (F). A value $<1$ indicated synergistic inhibition. The data represent mean \pm SEM, summarised from three independent experiments. ${ }^{*} \mathrm{p}<0.05,{ }^{* *} \mathrm{p}<0.01,{ }^{* * *} \mathrm{p}<0.001$, compared to control or untreated cells. $\# \mathrm{p}<0.05, \# \# \mathrm{p}<0.01$, \#\#\# p<0.001 compared to the corresponding gemcitabine treatment.

\subsubsection{FRAX597 and 5-FU synergistically inhibited MiaPaCa-2 and PANC-1 pancreatic cancer cell growth}

The combined effects of FRAX597 and 5-FU on proliferation were measured by incubation of PANC-1 and MiaPaCa-2 cells with increasing concentrations of 5-FU, alone or in combination with FRAX597, at a concentration close to the $\mathrm{IC}_{50}$ value given in Table 4.3.7. 5-FU alone, inhibited proliferation in a dose dependent manner with an IC50 value of $100 \mu \mathrm{M}$ for MiaPaCa2 and $130 \mu \mathrm{M}$ for PANC-1 (Table 4.3.9). A further reduction of proliferation was observed when treated with the combination of FRAX597 and 5-FU, compared to 5-FU alone (Fig. 4.3.9A-B). The combined effect of FRAX597 and 5-FU was shown to be synergistic $(\mathrm{CI}<1)$ when analysed using the Chou-Talalay method for PANC-1 and MiaPaCa-2 (Fig. 4.3.9C). These results demonstrated that FRAX597 and 5-FU synergistically inhibited pancreatic cancer cell growth. 

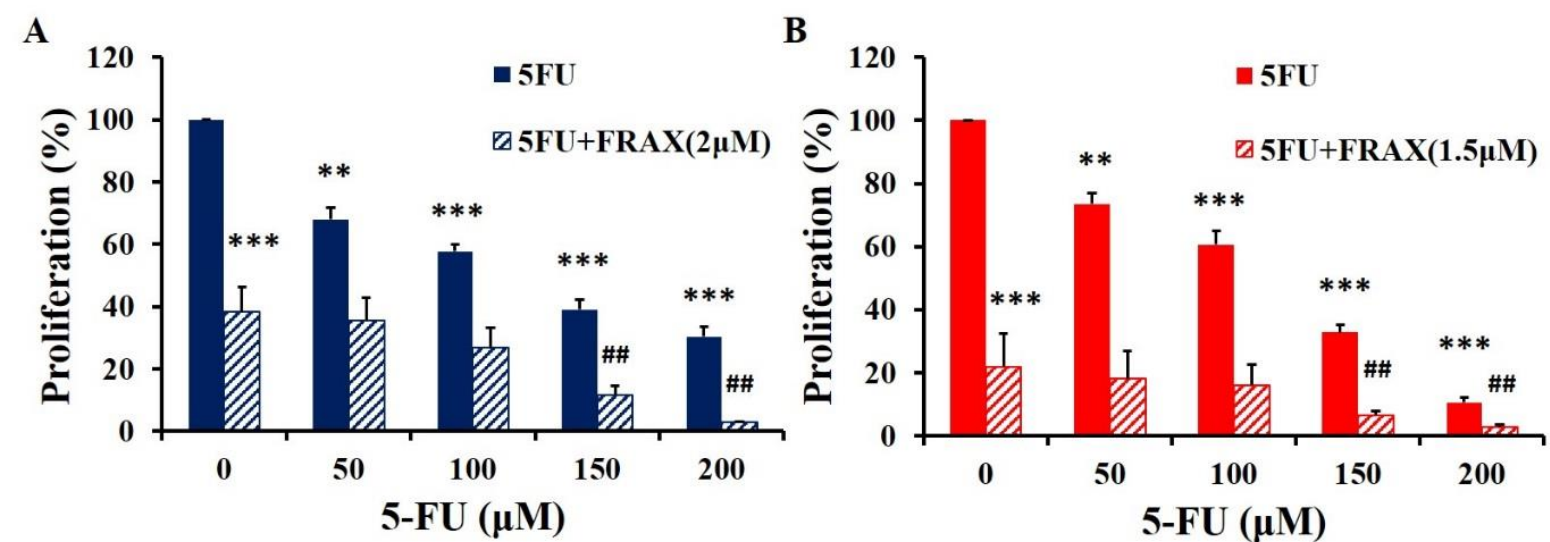

C

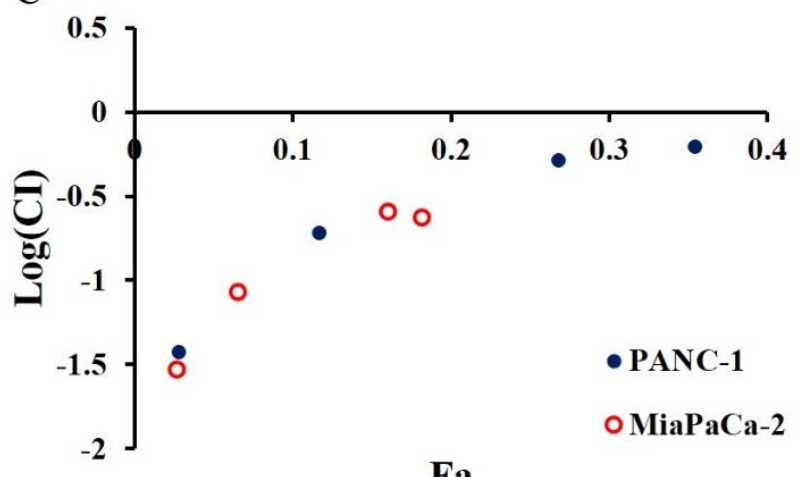

Fa

Figure 4.3.9 FRAX597 synergises with 5-FU to inhibit pancreatic cancer proliferation. The effects of 5-FU alone (5-FU, solid bars), and 5-FU with FRAX597 (5-FU + FRAX, striped bars), on proliferation of PANC-1 (A) and MiaPaCa-2 (B) cells were assessed by MTT assay. The concentration of FRAX597 used was based on the $\mathrm{IC}_{50}$ value determined in Chapter 4.3.7. The combination index (CI), calculated by the Chou-Talalay method, was used to determine the mechanism of action of FRAX597 and 5-FU (C). A value $<1$ indicated synergistic inhibition. The data represent mean \pm SEM, summarised from three independent experiments. ${ }^{* *} \mathrm{p}<0.01,{ }^{* * *} \mathrm{p}<0.001$, compared to control or untreated cells. \#\# $\mathrm{p}<0.01$ compared to the corresponding 5-FU treatment. 
Table 4.3.9: Proliferation $\mathrm{IC}_{50}$ values for 5-FU

$$
\begin{array}{cc}
\mathrm{IC}_{50}(\mu \mathrm{M}) \\
\text { MiaPaCa-2 } & \text { PANC-1 }
\end{array}
$$

$\overline{5-F U} \quad 100 \pm 20 \quad 130 \pm 35$

\subsubsection{Inhibition of pancreatic cancer cell growth by FRAX597 and gemcitabine is associated with reduced amounts of active PAK1}

The total amount of PAK1 and the amount of active PAK1 (pPAK1) was measured using Western blot after treatment with FRAX597 or gemcitabine, or the combination of FRAX597 and gemcitabine. The amount of active PAK1 was significantly reduced when treated with FRAX597 alone compared to control in all pancreatic cancer cell lines without affecting the amount of total PAK1 (Fig. 4.3.10A-E). No effect on PAK1 expression was observed when treated with gemcitabine alone. For MiaPaCa-2 cells (Fig. 4.3.10B) and BxPC-3 cells (Fig. 4.3.10C), combined treatment with FRAX597 and gemcitabine resulted in significant further reduction of active PAK1 compared to the FRAX597 treatment alone. In contrast, in the other cell lines PANC-1 (Fig. 4.3.10A), Pan02 (Fig. 4.3.10D) and LM-P (Fig. 4.3.10E), no further reduction in active PAK1 expression was observed following the combination treatment. These data suggested that FRAX597 and gemcitabine synergistically reduced pancreatic cancer cell growth by reducing the amount of active PAK1. 
A

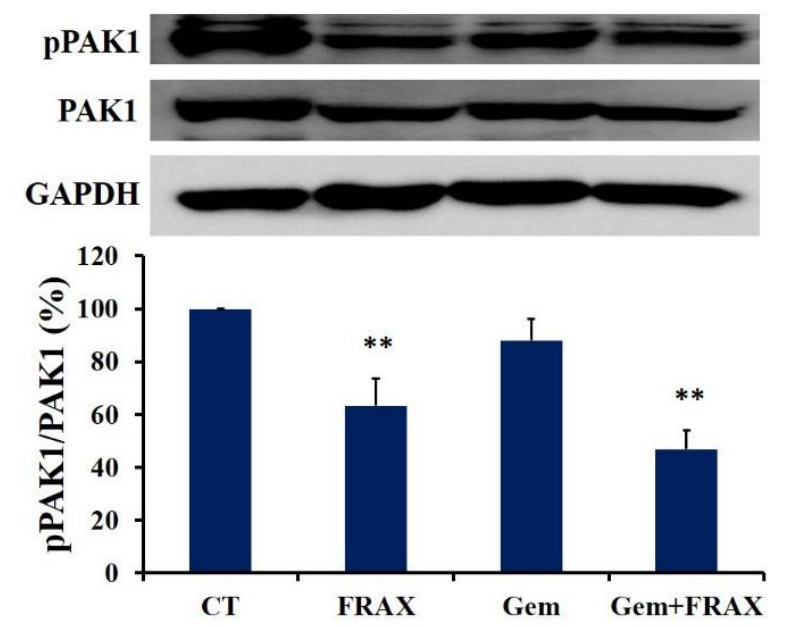

C
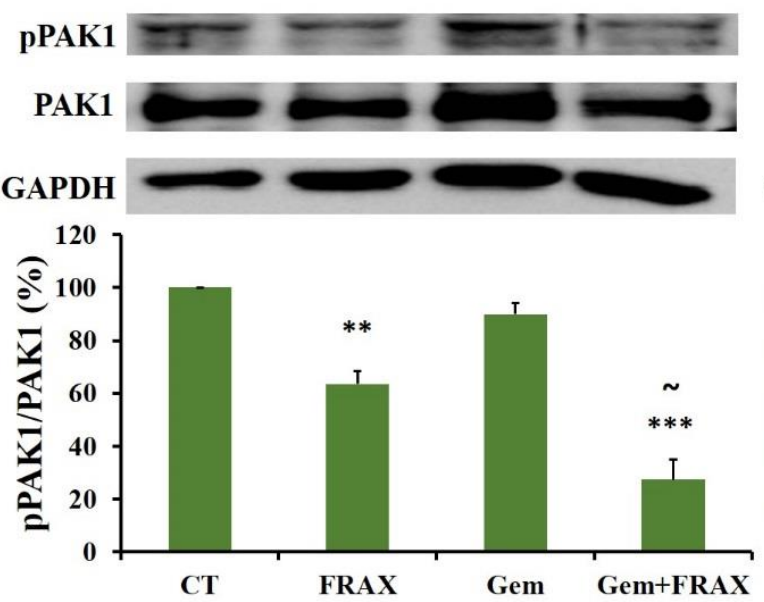

$\mathbf{E}$
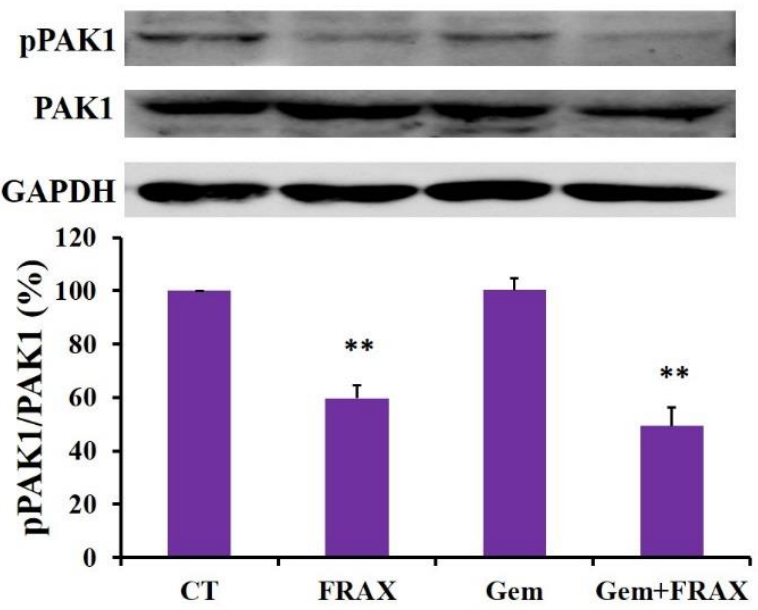

B
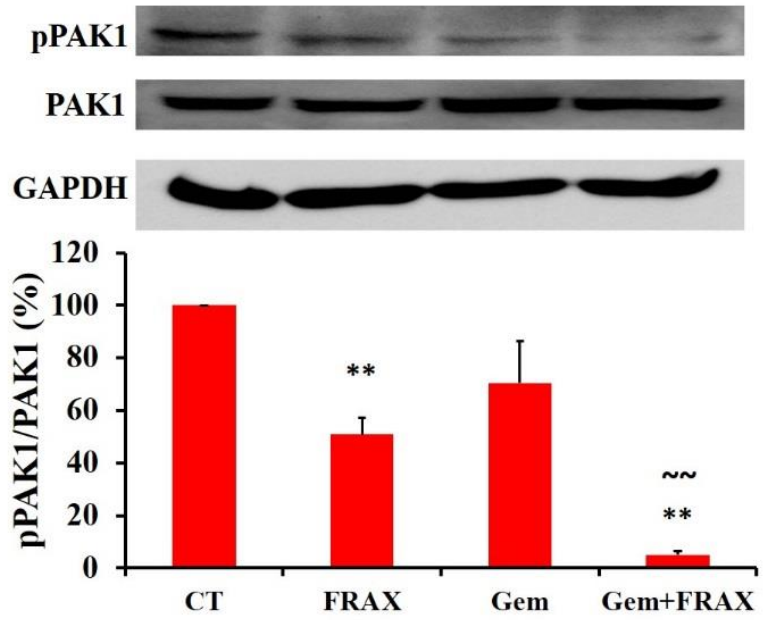

D
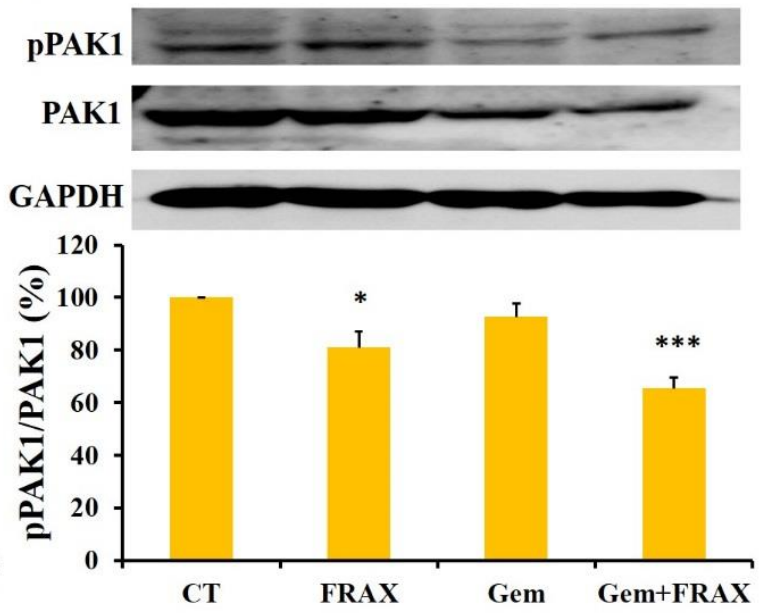

Figure 4.3.10 FRAX597 and gemcitabine reduced PAK1 activity. Expression of active PAK1 (pPAK1) and total PAK1 was measured in PANC-1 (A), MiaPaCa-2 (B), BxPC-3 (C), Pan02 (D), and LM-P (E) cells in the presence of FRAX597 (FRAX), gemcitabine (Gem), or 


\section{CHAPTER 4}

the combination of FRAX597 and gemcitabine (Gem + FRAX) using Western blot. Variations in protein loading were corrected by GAPDH expression, and the values for untreated control cells were taken as 100\%. FRAX597 treatment reduced PAK1 activity in all pancreatic cancer cell lines compared to untreated cells where a further reduction of PAK1 activity was observed in MiaPaCa-2 (B) and BxPC-3 (C) cells when gemcitabine was added to FRAX597. The data represent mean \pm SEM, summarised from three independent experiments. ${ }^{* *} \mathrm{p}<0.01$ $\mathrm{p}<0.001$ compared to control or untreated cells. $\sim \mathrm{p}<0.05, \sim \mathrm{p}<0.01$ compared to FRAX597 treatment.

\subsubsection{FRAX597 and gemcitabine inhibited pancreatic tumour growth and improved survival in orthotopic murine models}

The tumour taken rate was $100 \%$ for both pancreatic head and pancreatic tail models. The survival rate following surgery was $100 \%$ for the pancreatic tail model and over $95 \%$ for the pancreatic head model. There was no reported toxicity in any of the mice throughout the study. No difference in tumour volume was observed for mice treated with control or FRAX597 alone (Fig. 4.3.11A). Mice treated with gemcitabine alone had significantly reduced tumour volume when compared to control or FRAX597 alone, and a further significant reduction in tumour volume was observed for the mice treated with the combination of FRAX597 and gemcitabine. A similar trend was found when mice were evaluated for the presence of peritoneal carcinomatosis. $43 \%$ of mice in the combined treatment group had peritoneal carcinomatosis compared to $71 \%$ of mice in the gemcitabine treatment group, and all (100\%) mice in the control and FRAX597 treatment groups (Fig. 4.3.11B).

Survival of mice in the combination treatment group was significant increased compared to the control group, as assessed by stratified Cox regression analyses (Fig. 4.3.11C). A rates ratio of 
7 was calculated, indicating that mice in the control group had a mortality rate 7 times greater than mice in the combination treatment group (Table 4.3.11). The gemcitabine alone group had a rates ratio of 2.7 , compared to mice in the combination treatment group, however, was not statistically significant $(\mathrm{p}=0.09)$. These results indicate that FRAX597 and gemcitabine synergistically reduced pancreatic cancer growth and metastasis and increased pancreatic cancer murine survival in vivo.

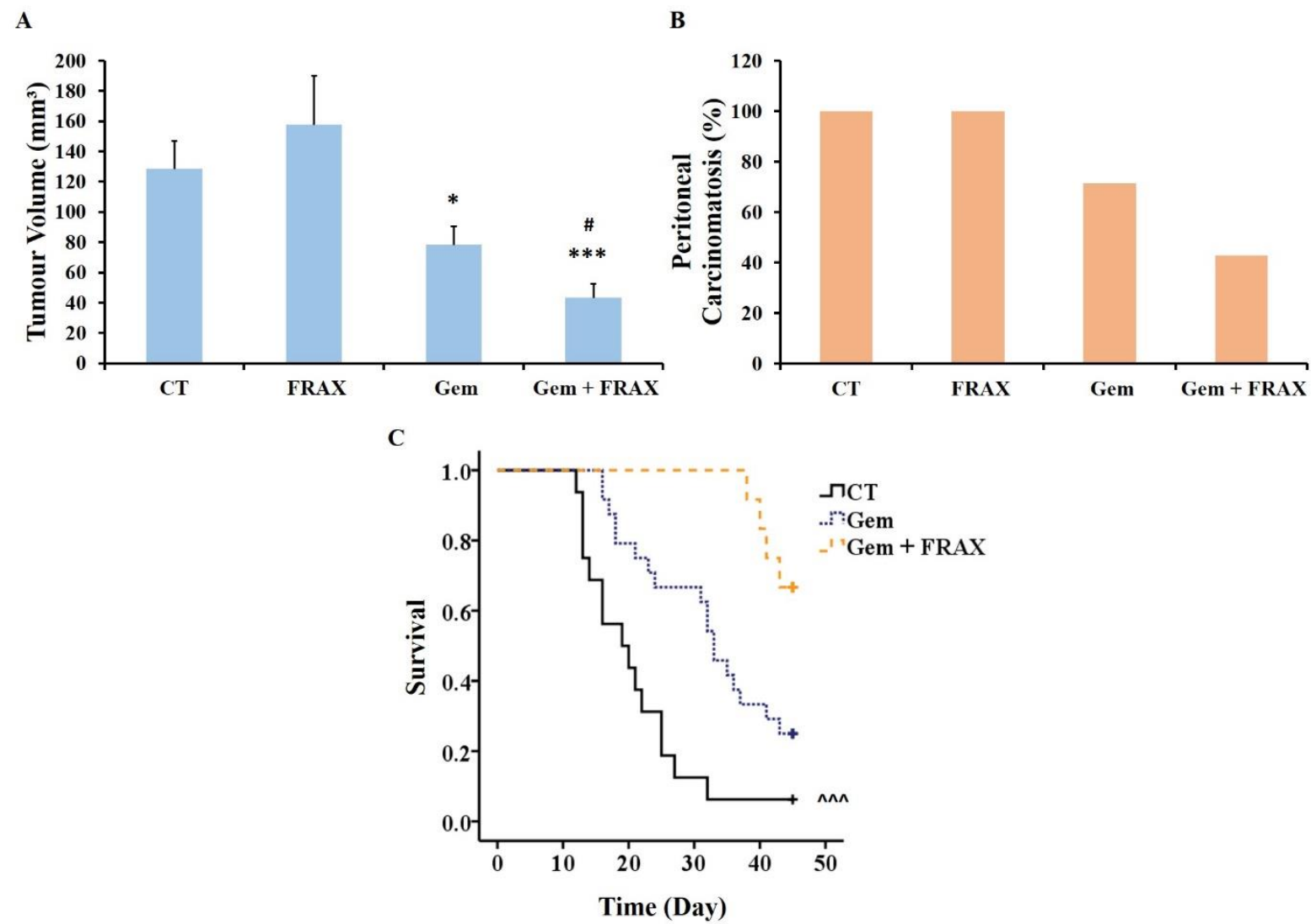

Figure 4.3.11 FRAX597 combined with gemcitabine inhibited tumour volume and increased survival in orthotopic murine models. Pan02 murine pancreatic cancer cells were injected orthotopically into the tail (A-B) or head (C) of the pancreas of C57Bl/6 mice. Mice were treated with saline (control; CT), FRAX597 (FRAX), gemcitabine (Gem), or FRAX597 and gemcitabine $($ Gem + FRAX). Mice were euthanased after 30 days for the orthotopic pancreatic tail model and tumour volumes were measured (A), and scored for the presence of 


\section{CHAPTER 4}

peritoneal carcinomatosis, or peritoneal spread (B). For assessment of survival, mice were euthanased after achieving a poor health score and the time to euthanasia plotted as a collated Kaplan-Meier curve $(\mathrm{C})$. The data represent mean \pm SEM. ${ }^{*} \mathrm{p}<0.05,{ }^{* * *} \mathrm{p}<0.001$ compared to control. \# $\mathrm{p}<0.05$ compared to gemcitabine treatment. ${ }^{\wedge} \wedge \wedge p<0.001$ compared to combination treatment using stratified Cox regression analysis.

Table 4.3.11: Cox regression survival analysis of treatment of FRAX597 and gemcitabine in a mouse orthotopic pancreatic cancer model

$\begin{array}{lll}\text { Treatment } & \text { Rates Ratio } \quad 95 \% \text { CI } \quad \text { p-value }\end{array}$

\begin{tabular}{lccc}
\hline Control & 7.0 & $1.8-27.0$ & 0.005 \\
Gemcitabine & 2.7 & $1.0-8.7$ & 0.09 \\
Gemcitabine + FRAX597 & $1.00($ ref $)$ & &
\end{tabular}

The overall statistics for the stratified Cox regression analysis were: $\chi 2(2)=9.9, p=0.007$

\subsubsection{FRAX597 and gemcitabine reduced tumour growth in orthotopic murine tumours by inhibiting proliferation}

Proliferation of the orthotopic tumours were measured by Ki67 IHC staining. Quantitative analysis was not undertaken due to the differences in tumour size and tumour cells could not be differentiated with stroma cells but qualitative comparisons were made. Little to no positive staining was observed in tumours treated with the combination of FRAX597 and gemcitabine compared to untreated control tumours and FRAX597-treated tumours which had higher amounts of positive staining (Fig. 4.3.12). Tumours treated with the combination of 


\section{CHAPTER 4}

gemcitabine and FRAX597, and gemcitabine alone had qualitatively, very similar staining pattern, showing that gemcitabine treatment decreased proliferation. These results indicate that FRAX597 and gemcitabine synergistically inhibited pancreatic cancer growth in vivo by reducing proliferation.
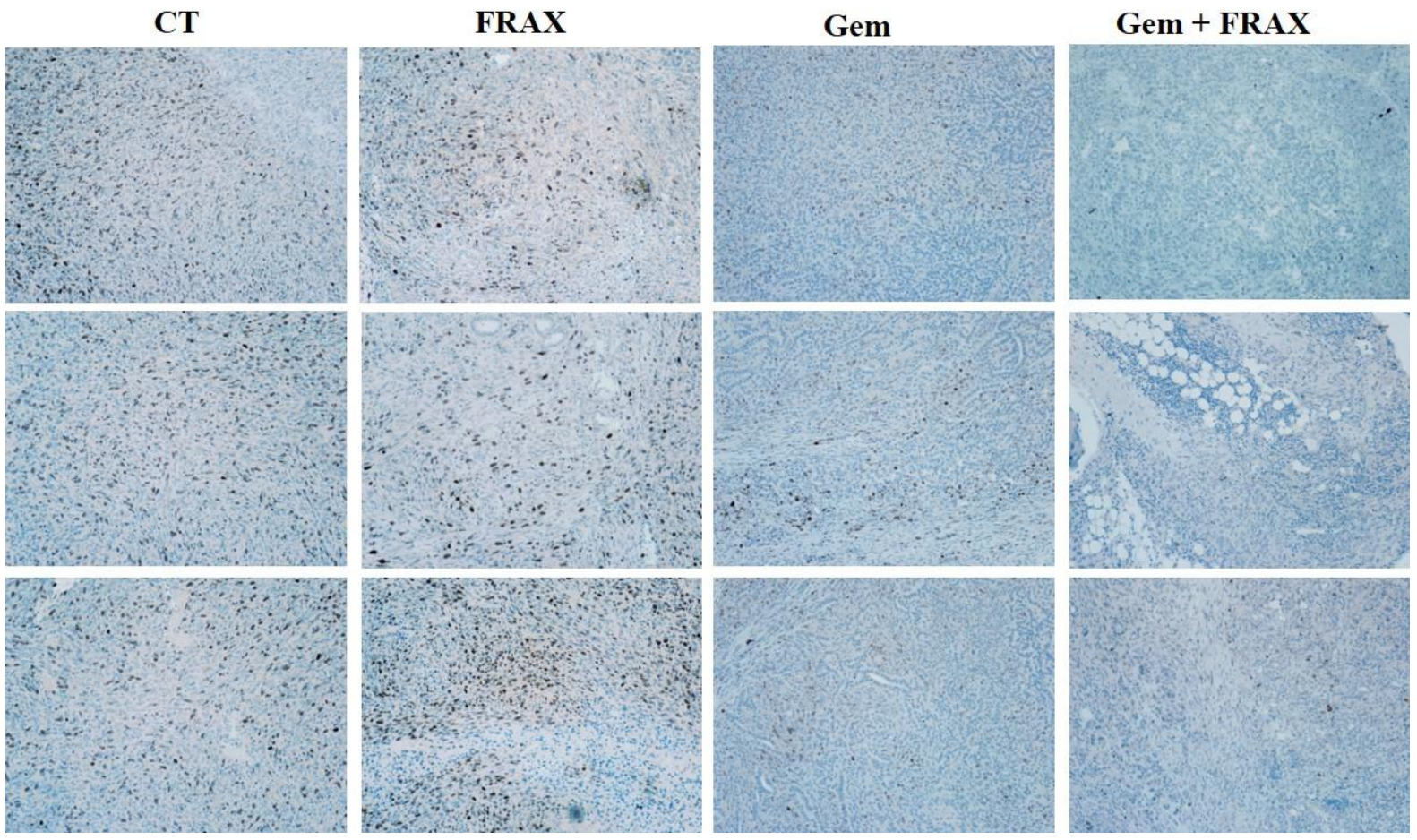

Figure 4.3.12 FRAX587 and gemcitabine inhibited tumour proliferation. Pan02 tumours from the orthotopic pancreatic tumour model treated with saline (control; CT), FRAX597 (FRAX), gemcitabine (Gem), or FRAX597 and gemcitabine (Gem + FRAX) were fixed and stained with Ki67. Gemcitabine alone and combination treated tumours had reduced Ki67 staining, with very little positive staining, compared to control. FRAX597 treated tumours had similar Ki67 staining compared to control tumours. 3 representative images were taken from each treatment group at 100x magnification. 


\subsubsection{FRAX597 and gemcitabine reduced collagen deposition in orthotopic murine}

\section{tumours by decreasing activated stellate cells}

The stroma was examined using picrosirius red staining which visualises the collagen deposition. A decrease in collagen staining was observed in both FRAX597-treated tumours and the combination treated tumours compared to untreated and gemcitabine-treated tumours where dense and numerous collagen fibres was observed near the leading edge of the tumour (Fig. 4.3.13). Stellate cell markers, $\alpha \mathrm{SMA}$ and desmin, had less intense positive staining in the combination treated tumours compared to the untreated, FRAX597 alone and gemcitabine alone tumours. These results suggest that FRAX597 and gemcitabine reduced the fibrotic content in the tumours by inactivating stellate cells resulting in reduced pancreatic cancer growth and improved survival in vivo.

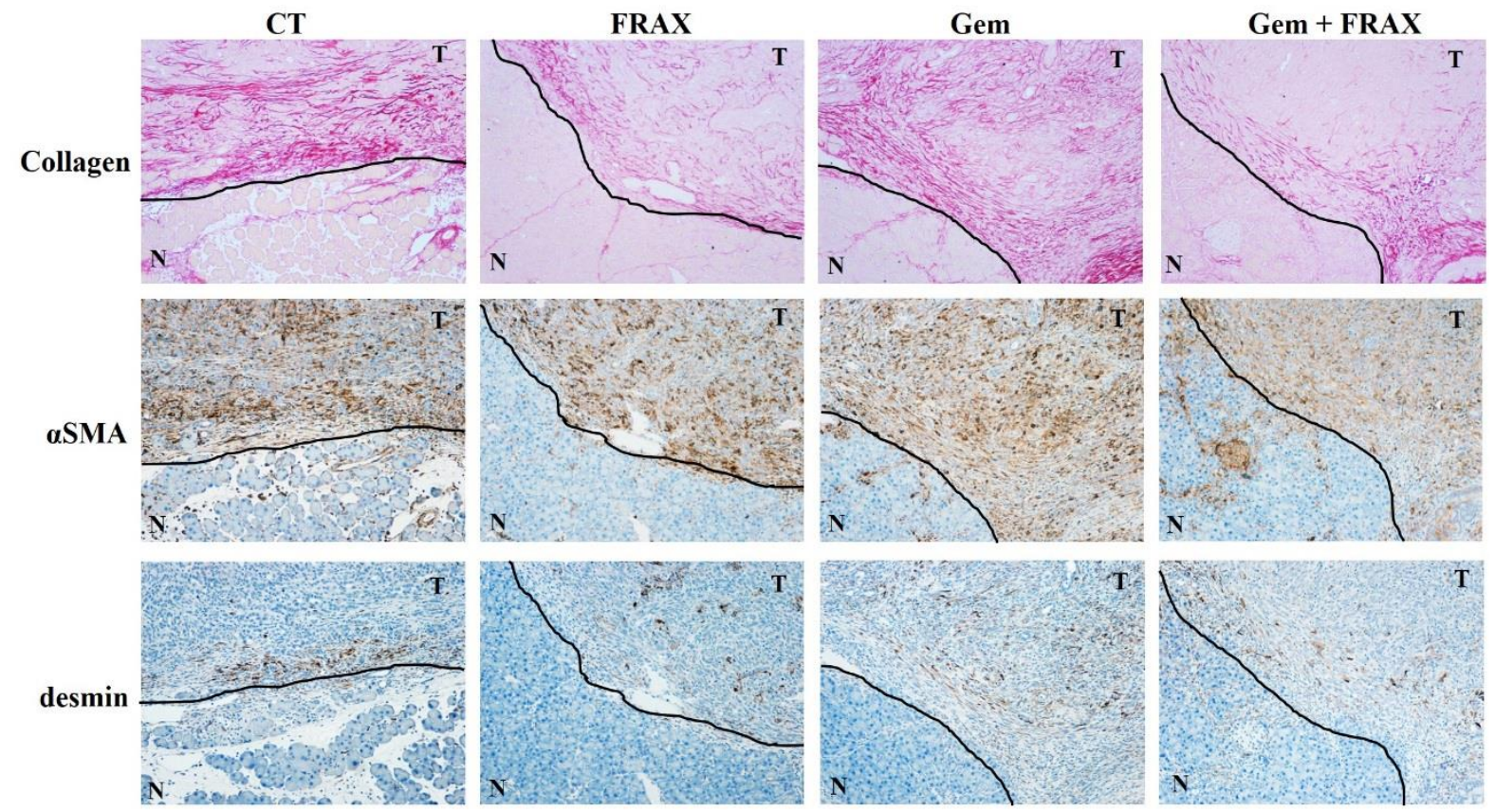

Figure 4.3.13 FRAX587 and gemcitabine reduced collagen deposition by inactivating stellate cells. Pan02 tumours from the orthotopic pancreatic tumour model treated with saline (control; CT), FRAX597 (FRAX), gemcitabine (Gem), or FRAX597 and gemcitabine (Gem + FRAX) were fixed and stained for collagen by picrosirus red staining and IHC staining with 
CHAPTER 4

stellate cell markers: $\alpha$ SMA and desmin. FRAX597 alone and combination treated tumours had decreased collagen staining compared to untreated and gemcitabine-treated tumours. The combination treated tumours had decreased and less intense aSMA and desmin staining compared to the untreated, FRAX597 alone and gemcitabine alone tumours. A representative image was taken from each treatment group at 200x magnification. 


\section{CHAPTER 4}

\subsection{Discussion}

The finding that PAK1 is expressed in pancreatic cancer cells is in agreement with other studies $(75,102)$. PAK1 was confirmed to not be expressed in normal pancreatic acinar or ductal cells, which are the likely progenitors of pancreatic cancer (261). It was also confirmed that islet cells stained positive for PAK1 where PAK1 has been implicated in insulin secretion in homeostatic conditions (229). PAK1 was expressed in the tumour tissue, in which the pancreatic cancer cells stained positive (Fig. 4.3.1A). Examination of the histopathological and patient data did not reveal any conclusive correlation with the intensity and amount of PAK1 staining, however, larger numbers and more comprehensive analyses are needed. All pancreatic cancer cell lines showed upregulation of PAK1 compared to the normal pancreatic ductal epithelial cell line, HPDE. The upregulation of PAK1 in the BxPC-3 and Pan02 cell lines, which are KRAS wildtype, indicates that PAK1 may be activated by KRAS-independent mechanisms. Further investigation will be required to elucidate the details of those mechanisms $(39,228)$. The increase in PAK1 expression implies that PAK1 is involved in pancreatic carcinogenesis, however, its role and therapeutic potential has not been fully elucidated.

Reduction of PAK1 expression by shRNA knockdown inhibited proliferation, sensitised cells to chemotherapy, and inhibited clonogenic growth of the PANC-1 and MiaPaCa-2 pancreatic cancer cell lines (Fig. 4.3.2-4). The two human cell lines were chosen based on the PAK1 activity where PANC-1 had 'high' activity whilst MiaPaCa-2 had 'low' activity (Fig. 4.3.1B). This difference in PAK1 activity may explain the contrasting results in cell survival where a reduction was observed in PANC-1 PAK1 KD cells (Fig. 4.3.2G) but not in MiaPaCa-2 PAK1 $\mathrm{KD}$ cells (Fig. 4.3.2H) indicating that 'high' PAK1 expressing cells may be driving cell survival via PAK1 but not in 'low' PAK1 expressing cells. It may also explain the difference in signalling proteins that were altered in the different PAK1 KD cell lines compared their 


\section{CHAPTER 4}

corresponding NC cells (Fig. 4.3.5G-H). Nevertheless, both PAK1 KD cell lines, regardless of their original PAK1 activity, observed a decrease in proliferation, further reduction in proliferation when treated with gemcitabine, and reduced tumourigenicity demonstrating the importance of PAK1 in those key cancer functions.

PAK1 KD cells had reduced tumourigenicity, which associates PAK1 signalling to stem-like properties in pancreatic cancer. PAK1 has been shown to be involved in resisting anoikis programing in breast epithelial cells through AKT signalling and also in hepatoma cells by hepatitis B virus $\mathrm{X}$ protein where resistance to anoikis represents a molecular basis for cancer progression, drug resistance and overall malignancy/tumourigenicity $(147,237)$. Furthermore, constitutively active PAK1 mutant renal carcinoma cells gained the ability to maintain sphere growth via PAK1-NFkB signalling (263). PAK1 has been shown to regulate NFkB signalling in pancreatic cancer (102). Together, this is the first study to demonstrate a role of PAK1 in stem-like properties and tumourigenicity in pancreatic cancer and indirect evidence suggests the involvement of the PAK1-NFkB signalling pathway.

The reduction in cell growth in PAK1 KD cells was associated with a decrease in AKT activity (Fig. 4.3.5A-B), but not in ERK activity (Fig. 4.3.5E-F). Our group has previously found that PAK1 stimulated growth of colorectal cancer cell lines via both ERK and AKT pathways (95), while another group has found that PAK1 signalled preferentially through the ERK pathway to control skin cancer growth (30). Thus, PAK1 signalling through AKT and ERK pathways is dependent on the cancer type, and our study suggests that PAK1 stimulates pancreatic cancer cell growth preferentially through the AKT pathway rather than the ERK pathway.

PAK1 may play a role in the resistance of pancreatic cancer to hypoxia through regulation of HIF $1 \alpha$. The transcription factor HIF $1 \alpha$ regulates oxygen delivery and metabolic adaptation to hypoxia and has been found to be a prognostic marker for pancreatic cancer (89). Pancreatic 


\section{CHAPTER 4}

tumours are known to be highly hypoxic, as they feature a dense stroma (desmoplastic reaction), which may contribute to pancreatic cancer invasion, metastasis, and resistance to therapy (247). Thus, mediators of survival in response to a hypoxic challenge are attractive therapeutic targets for pancreatic cancer. Although as far as we are aware, this is the first study to examine HIF1 $\alpha$ as a downstream effector of PAK1 in pancreatic cancer, PAK1 has previously been linked to HIF1 $\alpha$ in colorectal cancer (128). The ability of PAK1 to contribute to pancreatic carcinogenesis via multiple signalling pathways enhances its potential as a therapeutic target.

PAK1 knockdown also enhanced the sensitivity of PANC-1 and MiaPaCa-2 cells to gemcitabine (Fig. 4.3.3), as revealed by comparison of the $\mathrm{IC}_{50}$ values for inhibition of proliferation between control and knockdown clones (Table 4.3.3). Gemcitabine remains a standard monotherapy treatment for pancreatic cancer patients and there is interest in combining treatments with gemcitabine with the goal of decreasing chemotherapy-associated cytotoxicity, chemo-resistance and increasing survival (37). Previous studies have found that AKT and HIF1 $\alpha$, both shown to be PAK1 downstream effectors (Fig. 4.3.5), could play a role in gemcitabine resistance through $\mathrm{NF \kappa B}$ which limits gemcitabine uptake by decreasing nucleoside transporters such as hENT and hCNT (37). PAK1 has been shown to regulate NFkB signalling in pancreatic cancer and more recently, gemcitabine has been found to bind to the PAK1 active site to increase PAK1 activity resulting in gemcitabine resistance $(102,103)$. Synergism was observed with 5-FU, another chemotherapy, using the group 1 PAK inhibitor, FRAX597 (Fig. 4.3.9), suggesting that PAK1 sensitisation is not necessary specific to gemcitabine. Although, other chemotherapeutics such as nab-paclitaxel should also be tested, it does suggest a general sensitisation to chemotherapy. Together, the data supports the use of a PAK1 inhibitor combined with chemotherapy, most commonly gemcitabine, to limit chemotherapy cytotoxicity and chemo-resistance. 


\section{CHAPTER 4}

The group 1 PAK inhibitor, FRAX597, inhibited proliferation, migration/invasion, and survival in all pancreatic cancer cell lines tested (Fig. 4.3.7). Although FRAX597 has been found to also inhibit other kinases such as RET, YES1, TEK, and CSF1R, the similar results obtained in the PAK1 knockdown experiments suggest that in this case PAK1 is indeed the relevant target (123). Furthermore, the $\mathrm{IC}_{50}$ values for proliferation are similar to the values observed in NF2-null Schwann treated cells (123). However, the $\mathrm{IC}_{50}$ values for either proliferation or migration/invasion did not significantly correlate with the amount of active PAK1 in the pancreatic cancer cells (data not shown). This observation suggests that there may be a barrier (e.g. uptake at the cell membrane) that prevents realisation of the full potential for inhibition in intact cells. The presence of such a barrier could have contributed to the failure to detect any difference in tumour volume between the FRAX597-treated mice and the control mice in the in vivo study (Fig. 4.3.11A). The stroma may have also prevented the drug's uptake to the tumour, however, FRAX597-treated tumours had decreased collagen deposition suggesting an effect on the stroma with possible depletion and inactivation of stellate cells but no effect on tumour volume at that time-point (Fig. 4.3.13). This finding is the basis for investigating PAK1 in the stroma which will be explored and discussed in more detail in Chapter 5. These observations illustrate the importance of the microenvironment in assessment of a drug's efficacy, as the in vitro cell culture conditions may not fully mimic the clinical setting.

Combining the group 1 PAK inhibitor, FRAX597, with gemcitabine resulted in increased inhibition of PAK1 activity in some, but not all, of the pancreatic cancer cell lines tested (Fig. 4.3.10A-E). In all the pancreatic cancer cell lines tested, PAK1 activity was significantly decreased after treatment with FRAX597 alone, and no change in activity was observed after treatment with gemcitabine alone. Thus, the combination treatment of FRAX597 and gemcitabine expected to inhibit PAK1 to the same extent as FRAX597 treatment alone, as was 


\section{CHAPTER 4}

observed for PANC-1, Pan02 and LM-P cells. However, significantly greater inhibition was observed in MiaPaCa-2 and BxPC-3 cells after combination treatment, providing clear evidence for synergy, although the mechanism for this is unclear. Interestingly, these two pancreatic cancer cell lines had the lowest PAK1 activity of all the pancreatic cancer cell lines tested (Fig. 4.3.1B). As suggested above, low PAK1 activity cells may not rely on PAK1 signalling as found with the no difference in the survival of MiaPaCa-2 PAK1 KD cells, however, combining PAK1 inhibitors with gemcitabine, enhanced inhibitory effect of PAK1. This observation supports the use of PAK1 inhibitors with gemcitabine regardless of their active PAK1 status.

Treatment with FRAX597 combined with gemcitabine significantly decreased tumour volume in vivo (Fig. 4.3.11A) and revealed a promising trend towards decreasing metastasis (Fig. 4.3.11B) and increasing survival (Fig. 4.3.11C). Furthermore, Ki67 staining of the tumours indicated that the difference in tumour volume is due to inhibition of proliferation (Fig. 4.3.12). Although liver metastasis is often observed in the orthotopic pancreatic tail murine model over longer periods, only 3 mice from the control and FRAX treatment groups had liver metastases at sacrifice, and so no comparison could be undertaken (166). However, peritoneal carcinomatosis, or peritoneal spread, was present and was compared. As a difference in tumour volume was observed between animals treated with gemcitabine alone or with the combination of FRAX597 and gemcitabine, a decrease in peritoneal carcinomatosis and an increase in survival was expected, but significance was not reached. This may be due to the fact that the study was stopped early, before all mice developed advanced tumours. Although the potential clinical value of FRAX597 and the likely therapeutic benefit of targeting PAK1 are clearly established in vitro, longer studies are needed for a complete picture of the possible survival benefits of combination treatment. 


\section{CHAPTER 4}

\subsection{Conclusion}

PAK1 is upregulated in human pancreatic cancer and pancreatic cancer cell lines compared to normal pancreatic epithelial cells. Inhibition of PAK1 by knockdown using shRNA transfection indicated that PAK1 is required for cell proliferation, survival and clonogenic growth of human pancreatic cancer cell lines through AKT- and/or HIF1 $\alpha$-dependent pathway(s). Furthermore, PAK1 knockdown sensitised pancreatic cancer cells to gemcitabine. A group 1 PAK inhibitor, FRAX597, inhibited proliferation, migration/invasion, and survival of human pancreatic cancer cell lines by inhibition of PAK1 activity. When combined with gemcitabine and 5-FU, FRAX597 synergistically inhibited proliferation. FRAX597 and gemcitabine inhibited pancreatic cancer growth, metastasis, and improved survival in vivo. This study suggests the promise of inhibiting PAK1 function and outlines its potential value as a target for pancreatic cancer therapy in combination with gemcitabine. 
Chapter 5: Inhibiting PAK1 Suppresses Pancreatic Stellate Cell Activation and Increases Survival of Mice with Pancreatic Cancer. 


\section{CHAPTER 5}

\subsection{Introduction}

A leading hypothesis for the poor clinical outcome in pancreatic cancer is due to the presence of the stroma. The pancreatic cancer stroma is characteristic of pancreatic cancer. It has recently been an area of interest as it could potentially improve outcomes for patients with pancreatic cancer as targeting the stroma may result in increased sensitivity to current treatments. However, current studies are divided as to its main role in pancreatic carcinogenesis and how to develop therapies against the stroma to prevent further progression and improve patient outcomes.

The stroma, or desmoplastic reaction, is a dynamic milieu comprised of several different cell types (164). Pancreatic stellate cells (PSCs) are myofibroblast-like cells which produce the fibrosis that forms the majority of the stroma mass $(97,224,225)$. Although the technique for isolation of quiescent PSCs from rats and humans is well documented, there is little evidence of successful isolation from mice $(7,10)$. PSCs stain positively for desmin, vimentin and glial fibrillary acidic protein (GFAP) (170). In homeostasis, PSCs are quiescent and vitamin Acontaining lipid droplets are present in their cytoplasm. Upon pancreatic injury or in association with pancreatic cancer, PSCs lose their ability to store vitamin A and start to express alphasmooth muscle actin ( $\alpha \mathrm{SMA})$, transforming into an activated phenotype which has the ability to secrete excessive amounts of extracellular matrix (ECM) proteins such as collagen (49). In this study, the approach of measuring PSC activation by $\alpha$ SMA expression and PSC function by expression of desmin was adopted.

Targeting the stromal components of pancreatic cancer as a strategy to increase therapeutic response has thus far been unsuccessful with no clinically approved therapies (50). Stromal depletion by administration of IPI-926, which inhibits of the hedgehog pathway, resulted in depletion of tumour-associated stromal tissue, was investigated in mouse models and resulted 


\section{CHAPTER 5}

in increased mean vessel density with increased gemcitabine delivery and response, and a significant increase in median survival (169). These results was confirmed using another hedgehog inhibitor, AZD8542, in mouse models and led to the theory that the stroma was procarcinogenic (98), However, the randomised phase 2 clinical trial with IPI-926 was stopped early due to worse survival and more aggressive tumours (173). Furthermore, more recent mouse studies have suggested that the stroma may actually be confining and restraining the tumour (186). Prolonged myofibroblast depletion in a transgenic mouse model with deleted aSMA myofibroblasts resulted in more frequent precursor lesions (PanINs), and tumours were more commonly metastatic and highly proliferative (171). Also, patients with resected tumours with high stromal density had better survival after surgery compared to those with low stromal density who had more aggressive disease (207). A number of stroma-targeted therapies have yielded promising results in the preclinical setting and are currently under evaluation in clinical trials including PEGPH20 (86), which depletes hyaluronan, a component of the stromal ECM; TH-302 (19), a hypoxia-activated chemotherapeutic; modified vitamin D (195), which targets the vitamin D receptor on PSCs resulting in stromal reprogramming; and losartan (26), an angiotensin II type 1 receptor inhibitor which inhibits PSC activity. Although the biology of the stroma has not been fully elucidated, a complete understanding of the stroma concurrently

with the tumour biology will result in better targeted treatments with better therapeutic delivery, leading to improvements in patient survival for pancreatic cancer.

The bidirectional interaction between PSCs and pancreatic cancers is complex (Fig. 5.1). PSCs can secrete a variety of paracrine factors, such as growth factors, cytokines, and chemokines, which promote pancreatic cancer growth by stimulating proliferation and inhibiting apoptosis, and stimulate invasion and metastasis by inducing an epithelial to mesenchymal transition (EMT) $(78,176)$. Furthermore, in vitro studies have found that although pancreatic cancer has similar chemosensitivity to other cancers, patients with pancreatic cancer are less responsive 


\section{CHAPTER 5}

to chemotherapies and tumour cells incubated with stroma cells were more resistant to chemotherapy $(97,129)$. These observations highlight the importance of the fibrosis which results in collapsed or compressed intratumoural vasculature, and hypoxia contributing to chemotherapeutic resistance (145). On the other hand, pancreatic cancer cells can also secrete numerous factors to activate PSCs and promote their growth (154). The close and dynamic interaction between PSCs and pancreatic cancer cells suggests a cautious approach in translating the findings from in vitro studies, as PSCs may exert different effects depending on disease stage and site and may explain the conflicting conclusions reached in past studies.

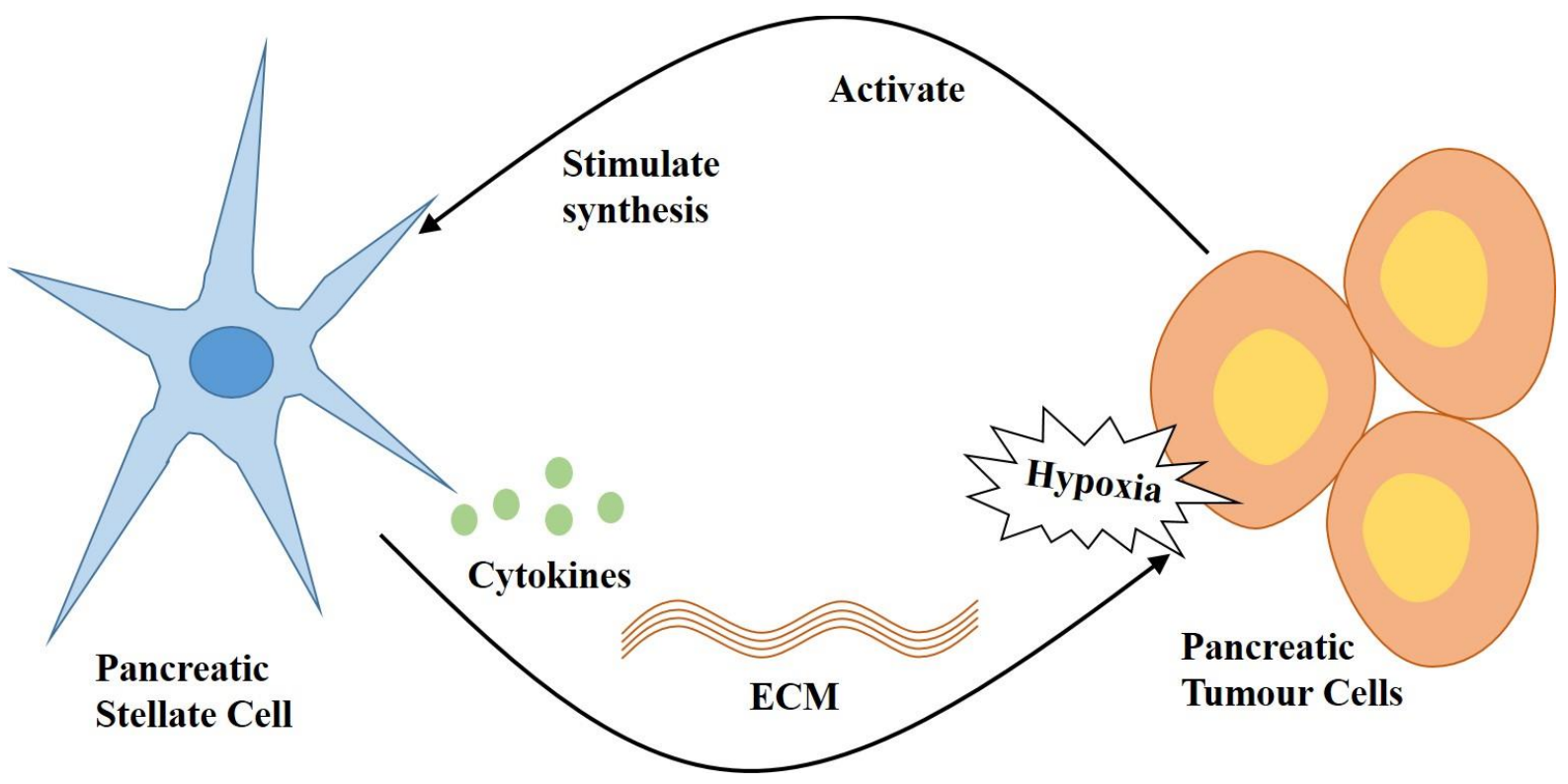

Figure 5.1 Simplified schematic diagram of the bidirectional interaction between pancreatic stellate cells and pancreatic tumour cells. PAK1 has been studied in the pancreatic tumour cells but has not been studied in context of the pancreatic stellate cells or their interactions.

The role of PAK1 has not, to our knowledge, been studied in the pancreatic cancer stroma or in PSCs. However, indirect evidence from studies investigating PAK1 in pancreatic cancer 
cells have found the involvement of the MAPK and NFkB pathway, which has been implicated in PSC activation, PSC secretion of cytokines, and PSC proliferation $(144,246)$. Furthermore, results from Chapter 4.3.13, suggests that PAK1 may be involved in PSCs with a reduction in collagen deposition in the tumours treated with the group 1 PAK inhibitor, FRAX597 and combination of FRAX597 and gemcitabine. FRAX597 alone treated tumours had no tumour volume reduction compared to control, suggesting that FRAX597 may have impeded stellate cell activation or activity at earlier time points which was overcome at the endpoint explaining the decrease in collagen deposition but no difference in stellate cell markers. However, the combination treated tumours had reduced collagen deposition and reduced staining intensity of stellate cell markers, demonstrating the effect of combining a PAK1 inhibitor with gemcitabine on the stroma and is indicative that PAK1 plays a role in the pancreatic stroma.

This study aimed to investigate the role of PAK1 in PSCs isolated from human pancreatic cancer specimens and to evaluate the potential of PAK1 as a therapeutic target in PSCs using the group 1 PAK inhibitor, FRAX597. The role of PAK1 in the interaction between PSCs and pancreatic cancer cells was investigated using conditioned media experiments, co-culture experiments, and orthotopic murine models in PAK1 knockout $(\mathrm{KO})$ mice. 


\section{CHAPTER 5}

\subsection{Methods}

The proliferation, hypoxia treatment of cells, and Western blot assays; orthotopic pancreatic cancer murine implantation, tissue processing, immunohistochemical staining procedures, and picrosirius red staining have been described in Chapter 2 .

\subsubsection{Cells}

Human pancreatic stellate cells (hPSC) were prepared by the outgrowth method (10) and as described in Chapter 2.2. PSC purity was determined by IHC, as described in Chapter 2.16, for aSMA, GFAP and desmin, as well as morphology (spindle-shaped cells with cytoplasmic extensions). Only cells that were negative for cytokeratin 19 (CK19) were used, to exclude tumour contamination. 6 human tissue were obtained and PSC isolation attempted. 2 were unsuccessful with one expressing CK19 expression (epithelial contamination) and the other lost due to bacterial/fungal contamination.

PAK1 KD cells was also attempted in hPSC1 cells but due to the nature of stellate cells, stellate cells do not survive following colony selection. As such, hPSC1 cells were transfected with one of the three SureSilencing shRNA plasmids for human PAK1 (IS2, IS3, and IS4), or with a scrambled sequence as a negative control (NC) as described in Chapter 2.3.6 $6 \mathrm{~g}$ of plasmid DNA, or NC, was added to the cells for 24 hours, then allowed to grow to confluency and tested for PAK1 protein expression by Western blot as described in Chapter 2.10.

\subsubsection{Cell Apoptosis Assay}

Cell apoptosis was assessed using the annexin V-FITC apoptosis assay as described in Chapter 2.7. hPSC1 cells were seeded in 6-well plates and treated with FRAX597 at a single concentration based on the proliferation $\mathrm{IC}_{50}$ for 24 hours. Roughly $2 \times 10^{5}$ cells were then 


\section{CHAPTER 5}

collected, spun down and resuspended in $500 \mu \mathrm{l}$ of $1 \mathrm{X}$ binding buffer, annexin V-FITC and propidium iodide (PI) was added and cells analysed by flow cytometry.

\subsubsection{Cell Migration/Invasion Co-culture}

Tumour cell migration/invasion was measured in the presence of hPSC1 cells using a coculture Transwell Boyden Chamber assay, a variation of the Transwell Boyden Chamber assay as described in Chapter 2.8. hPSC1 cells were seeded in the lower chamber at increasing ratios to PANC-1 or PANC-1 KD cells for 1 hour before the upper insert was placed. PANC-1 and PANC-1 KD cells were seeded in the upper chamber at $2 \times 10^{4}$ cells, so hPSC1 cells were seeded at $0,2 \times 10^{2}$ (for ratio of 0.01 ), $2 \times 10^{4}$ (for ratio of 1 ), and $1 \times 10^{6}$ (for ratio of 50 ). Cells were incubated for 24 hours before cells were fixed and stained as per described.

\subsubsection{Cell Proliferation using conditioned media}

Cell proliferation was measured as described in Chapter 2.4, except conditioned media from PANC-1 KD cells were used. PANC-1 KD cells were seeded at similar concentrations in 24well plates for 48 hours. Media was then collected, spun down and the supernatant was directly added to hPSC1 cells, which had been seeded 24 hours prior in a 96 -well plate, with $1 \mu \mathrm{Ci} /$ well [methyl- ${ }^{3} \mathrm{H}$ ]-thymidine. hPSC1 cells were then collected and harvested at 24-, 48-, and 72-hour time points and growth curves were fitted using a log-scale (MATLAB).

\subsubsection{Orthotopic Pancreatic Cancer Murine Study Design}

Pan02 (2.5 x $10^{5}$ cells) were suspended in $50 \mu \mathrm{L}$ of Matrigel $^{\mathrm{TM}}$ matrix solution (BD Biosciences) and implanted into the pancreatic head or pancreatic tail of $\mathrm{C} 57 \mathrm{~B} 1 / 6$ mice or $\mathrm{C} 57 \mathrm{Bl} / 6 \mathrm{PAK} 1$ knockout (KO) mice as described in Chapter 2.13. Tumour leakage was not observed in any mice. Mice were monitored based on health score for up to 30 days for the pancreatic head model and for up to 37 days for the pancreatic tail model and euthanised when a poor health score was reached. 


\section{CHAPTER 5}

In the pancreatic head model, assessment of murine survival was undertaken over four studies. A total of $17 \mathrm{C} 57 \mathrm{Bl} / 6$ mice (WT) and $22 \mathrm{C} 57 \mathrm{Bl} / 6 \mathrm{PAK} 1 \mathrm{KO}\left(\mathrm{PAK}^{-/-}\right)$mice were used. A collated Kaplan-Meier survival curve was plotted (SPSS). 3 mice tumours from WT and PAK1'I- mice, that were euthanased at the same time point, were isolated, and their tissue processed as described in Chapter 2.14 for $\mathrm{H} \& \mathrm{E}$ and IHC staining and protein analysis.

In the pancreatic tail model, assessment of tumour growth and metastasis was undertaken where $9 \mathrm{C} 57 \mathrm{Bl} / 6$ mice (WT) and $9 \mathrm{C} 57 \mathrm{Bl} / 6 \mathrm{PAK} 1 \mathrm{KO}\left(\mathrm{PAK}^{-/-}\right)$mice were implanted. Tumour volume was measured and calculated as described in Chapter 2.13.

\subsubsection{Subcutaneous Flank Pancreatic Cancer Murine Study}

To examine tumour growth in real time, Pan02 $\left(2 \times 10^{6}\right.$ cells $)$ were subcutaneously implanted into the flank of $5 \mathrm{C} 57 \mathrm{Bl} / 6$ mice (WT) and $8 \mathrm{C} 57 \mathrm{Bl} / 6 \mathrm{PAK} 1 \mathrm{KO}\left(\mathrm{PAK} 1^{-/}\right)$mice. Tumours were monitored as described in Chapter 2.12. Mice were sacrificed on day 50. 


\section{CHAPTER 5}

\subsection{Results}

\subsubsection{PAK1 is expressed in pancreatic cancer stroma and in isolated pancreatic stellate}

cells

PAK1 staining was observed in the stromal component of human pancreatic cancer specimens (Fig. 5.3.1A). Stromal PAK1 staining could also be observed surrounding pancreatic precursor lesions: PanIN and IPMN. Myofibroblast-like cells were isolated from 6 specimens and 4 were successfully isolated from and confirmed to be PSCs by their positive staining for desmin, GFAP, and $\alpha$ SMA with strong PAK1 staining in the cytoplasmic compartment (Fig. 5.3.1B). The expression of PAK1, desmin and aSMA was compared by Western blotting to the human pancreatic stellate cell line, hPSC1. All cell lines expressed PAK1, $\alpha \mathrm{SMA}$ and desmin, although the relative amounts varied (Fig. 5.3.1C). The data indicate that PAK1 is present in the pancreatic cancer stroma and in PSCs and is suggestive that PAK1 plays a role in PSCs. 
A

PanIN

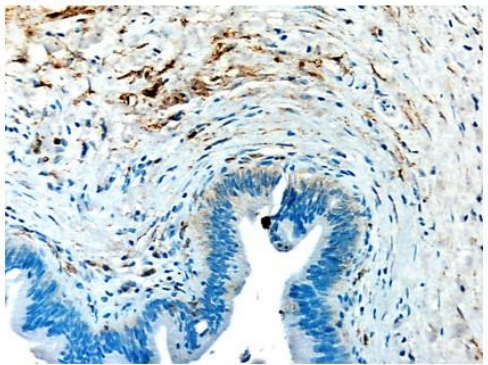

\section{IPMN}

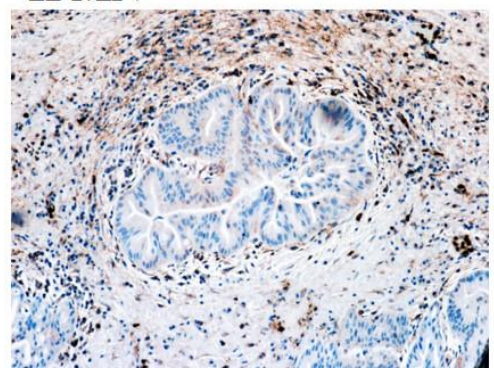

\section{Pancreatic Cancer}
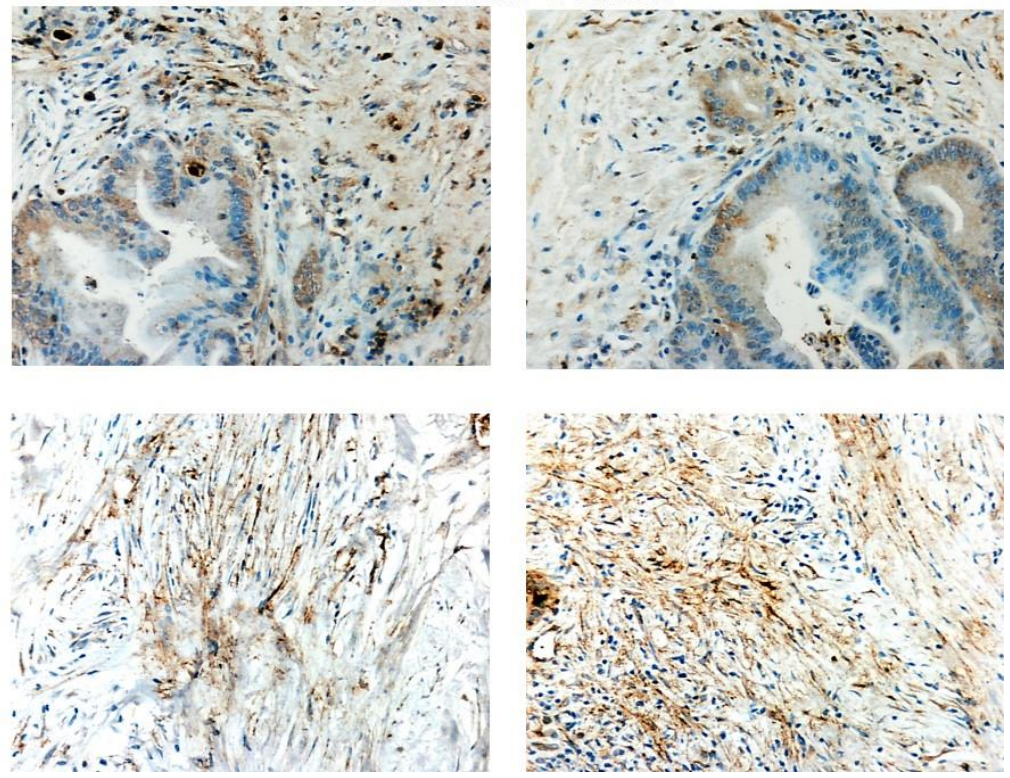

B

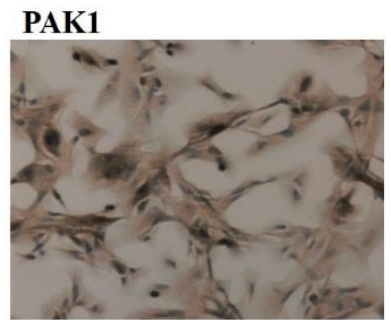

desmin

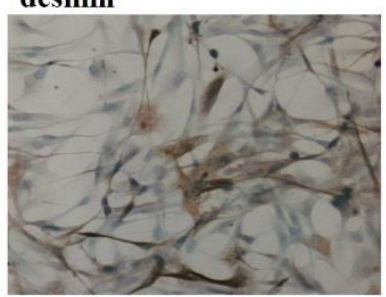

GFAP
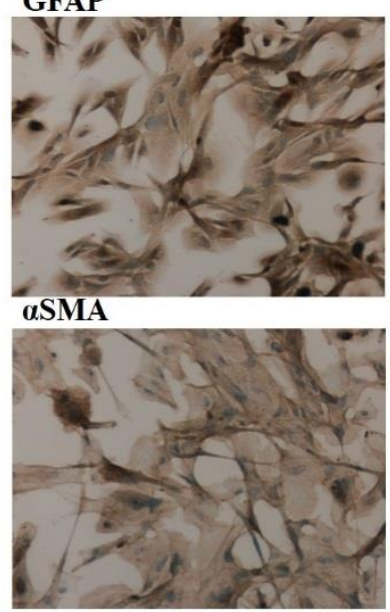

C

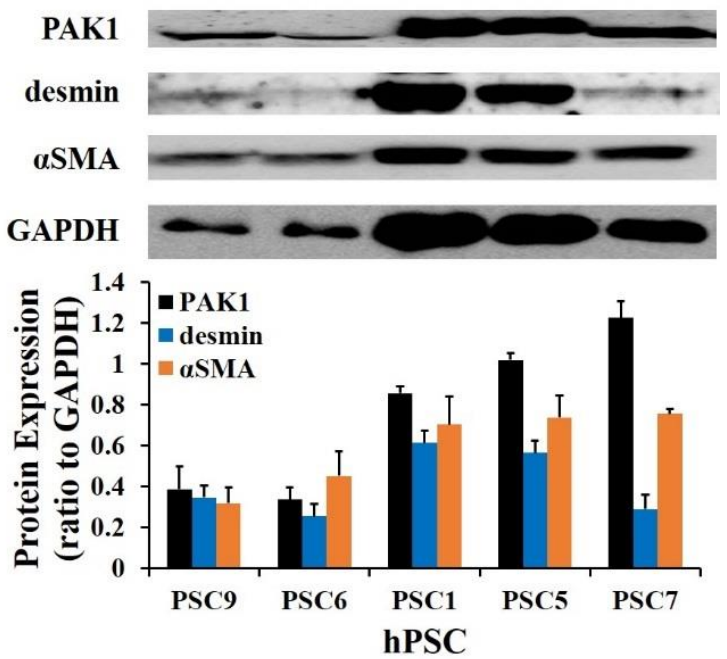

Figure 5.3.1 PAK1 is expressed in the stroma of human pancreatic cancer specimens and

in isolated pancreatic stellate cells. Representative images show the varying levels of PAK1 staining in the microenvironment of human pancreatic cancer specimens at 200x (top 3 images) and 100x (bottom 3 images) magnification (A). The purity of pancreatic stellate cells (PSC), isolated from human pancreatic cancer specimens, was confirmed by morphology, and by immunocytochemical staining for desmin, GFAP (glial fibrillary acidic protein) and $\alpha$ SMA 


\section{CHAPTER 5}

(alpha-smooth muscle actin) at 400x magnification (B). The human pancreatic stellate cell line hPSC1 and PSC isolated from 4 different human pancreatic cancer specimens expressed varying levels (ratio to GAPDH) of PAK1, desmin and $\alpha$ SMA as detected by Western blot (C). The data represent mean \pm SEM, summarised from three independent experiments.

\subsubsection{Inhibition of PAK1 by FRAX597 inhibited stellate cell activation, function and proliferation, and increased apoptosis}

The group 1 PAK inhibitor, FRAX597, significantly decreased PAK1 expression and activity (Fig. 5.3.2A) and significantly decreased $\alpha \mathrm{SMA}$ and desmin expression at $1 \mu \mathrm{M}$ and $2 \mu \mathrm{M}$ (Fig. 5.3.2B) in the hPSC1 cell line. FRAX597 also decreased cyclin D1 expression and inhibited proliferation in a dose-dependent manner with an $\mathrm{IC}_{50}$ of $0.61 \pm 0.28 \mu \mathrm{M}$ (Fig. 5.3.2C). At 1 $\mu \mathrm{M}$, FRAX597 induced PSC apoptosis with a 3.8-fold increase in apoptotic cells (from 2.7\% in untreated cells to 10.2\% in the FRAX597-treated cells) (Fig. 3.2.2D). These results indicated that inhibition of PAK1 by FRAX597 inhibited the activation of PSCs, decreased proliferation by inhibition of cyclin D1 expression, and increased apoptosis. 
CHAPTER 5

A

B
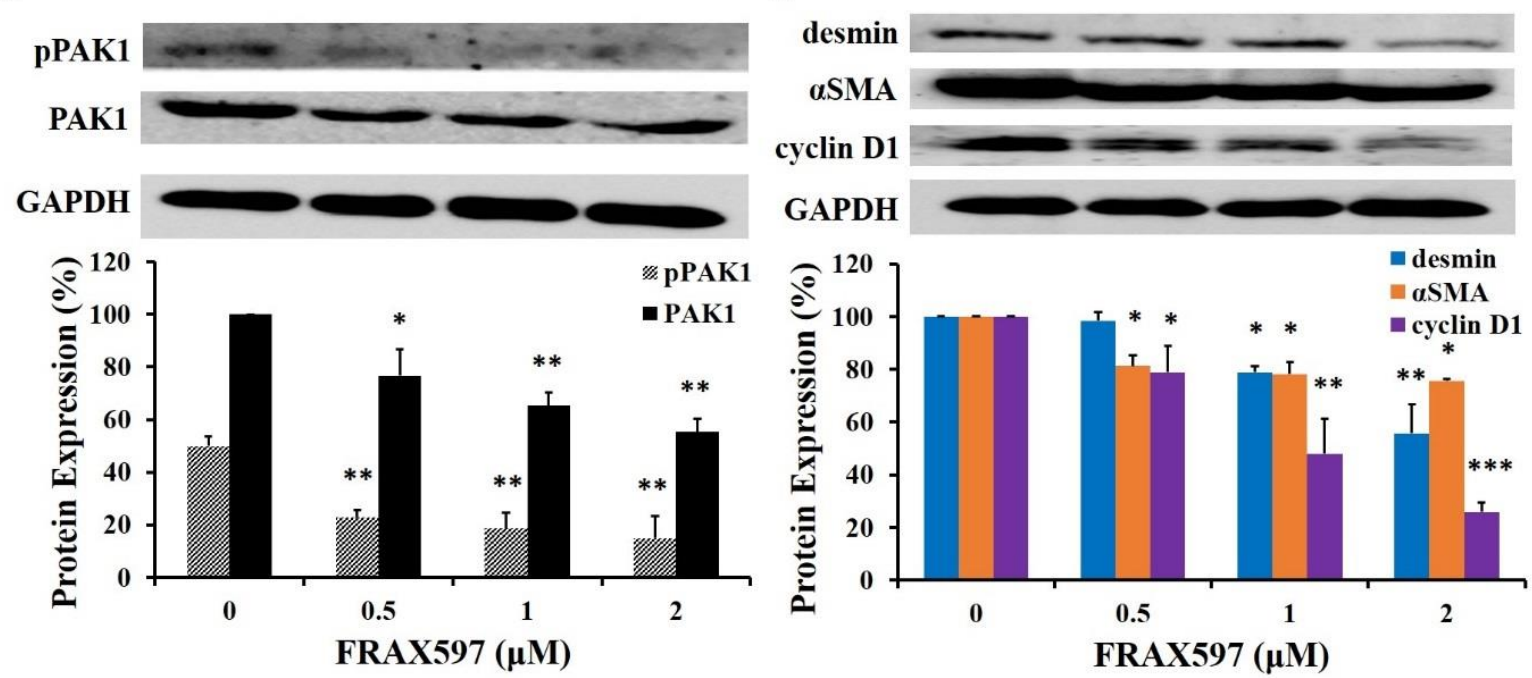

C

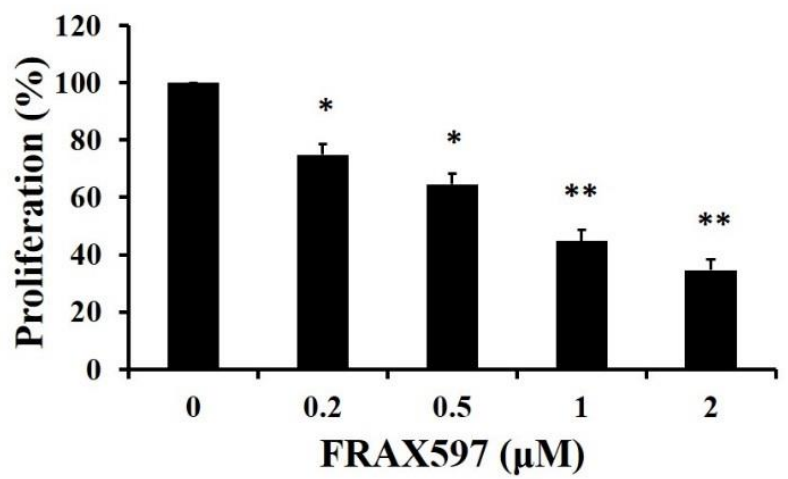




\section{CHAPTER 5}

D
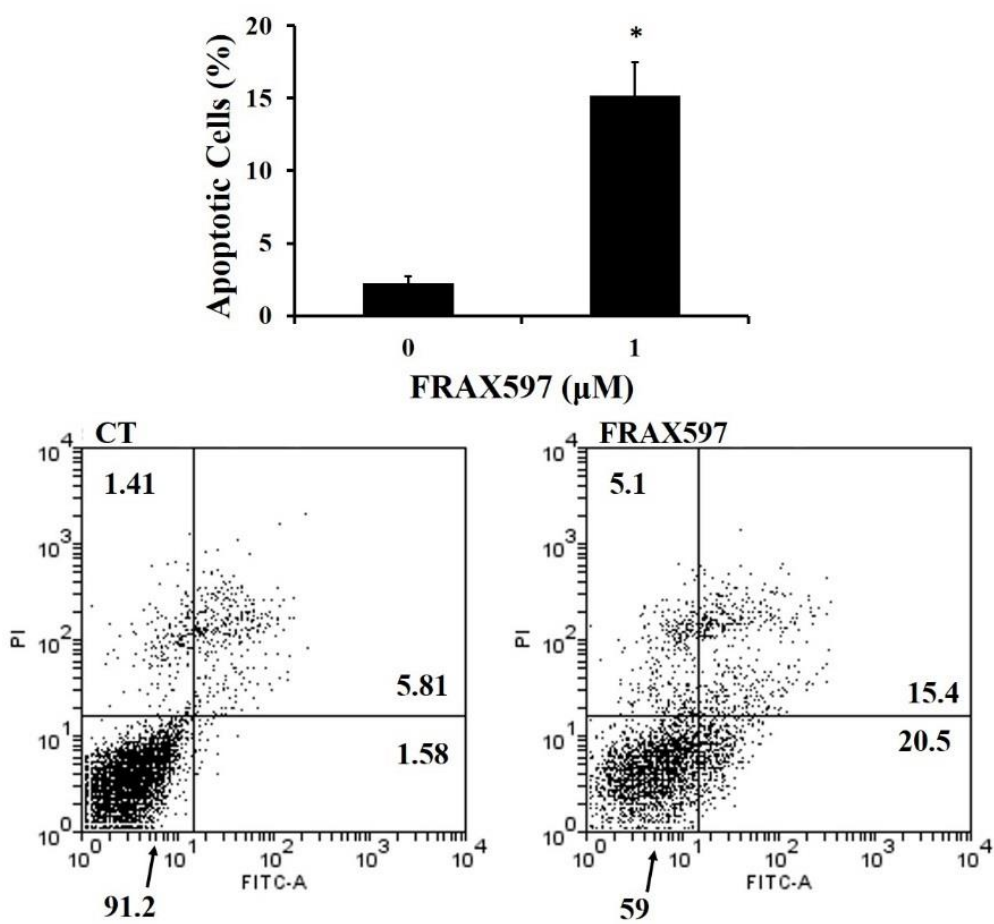

Figure 5.3.2 The group 1 PAK inhibitor, FRAX597, decreased PAK1 activity and expression and PSC activation and function, inhibited PSC proliferation, and increased apoptosis. FRAX597, a group 1 PAK inhibitor, reduced the active form of PAK1 (pPAK1) and total PAK1 expression (A), reduced stellate cell activation ( $\alpha$ SMA expression) and function (desmin expression), and decreased expression of the cell cycle promoter, cyclin D1 (B) in the hPSC1 cell line. FRAX597 inhibited proliferation in a dose dependent manner (C) and increased apoptosis (D). FRAX597 treatment increased the percentage of FITC $/ \mathrm{PI}^{-}$cells (apoptotic cells) compared to untreated (CT) cells. FITC-/PI' cells (lower left quadrant) are healthy cells, $\mathrm{FITC}^{+} / \mathrm{PI}^{-}$cells (lower right quadrant) are cells undergoing apoptosis, and $\mathrm{PI}^{+}$ cells (upper two quadrants) are dead cells. The data (mean \pm SEM, summarised from three independent experiments) are presented as a percentage to untreated cells, except for pPAK1 expression, which is presented as a percentage of the PAK1 value for untreated cells. ${ }^{*} \mathrm{p}<0.05$, $* * \mathrm{p}<0.01, * * * \mathrm{p}<0.001$ compared to untreated cells. 


\subsubsection{Hypoxia increased expression and activity of PAK1 in PSCs}

As found in Chapter 4.3.5, knockdown of PAK1 significantly reduced the hypoxia-induced HIF $1 \alpha$ expression observed in pancreatic cancer cell lines on exposure to hypoxia, suggesting PAK1 may play a role in the resistance of pancreatic cancer to hypoxia through regulation of HIF1 $\alpha$. The effect of PAK1 on in PSCs under hypoxia was investigated. PAK1 expression and activity, and aSMA expression, were significantly increased in the hPSC1 cell line under hypoxia (Fig. 5.3.3A). At a concentration of $1 \mu \mathrm{M}$, FRAX597 significantly inhibited PAK1 expression and activity, and $\alpha$ SMA expression under both normoxia and hypoxia. HIF $1 \alpha$ expression was elevated by hypoxia and this hypoxia-induced increment of HIF1 $\alpha$ was significantly inhibited by FRAX597 at $1 \mu \mathrm{M}$. The HIF1 $\alpha$ expression was also decreased by FRAX597 under normoxia (Fig. 5.3.3B). These results demonstrated a positive relationship between the expression and activity of PAK1, and the activation of PSCs and HIF1 $\alpha$ expression, in response to hypoxia. 


\section{CHAPTER 5}

A

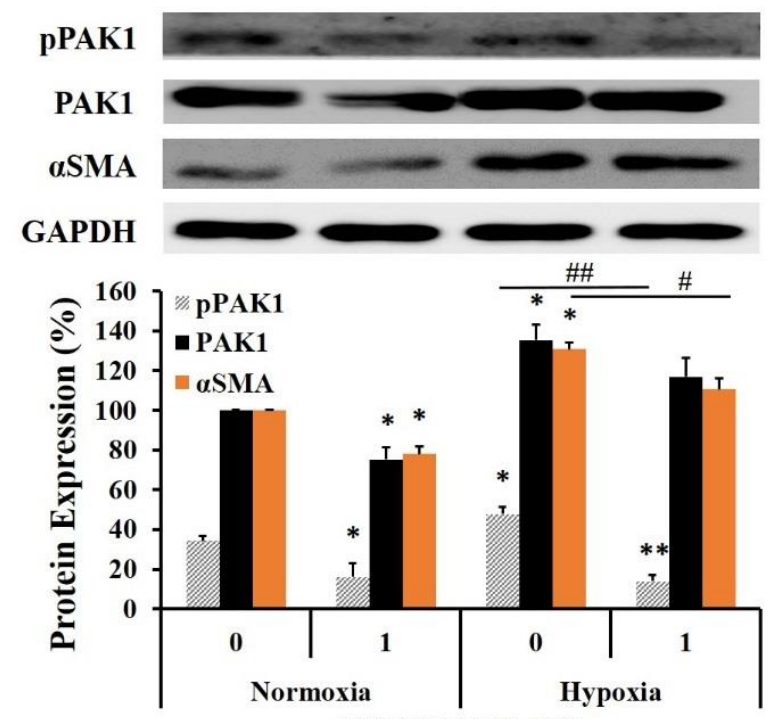

FRAX597 $(\mu \mathrm{M})$
B

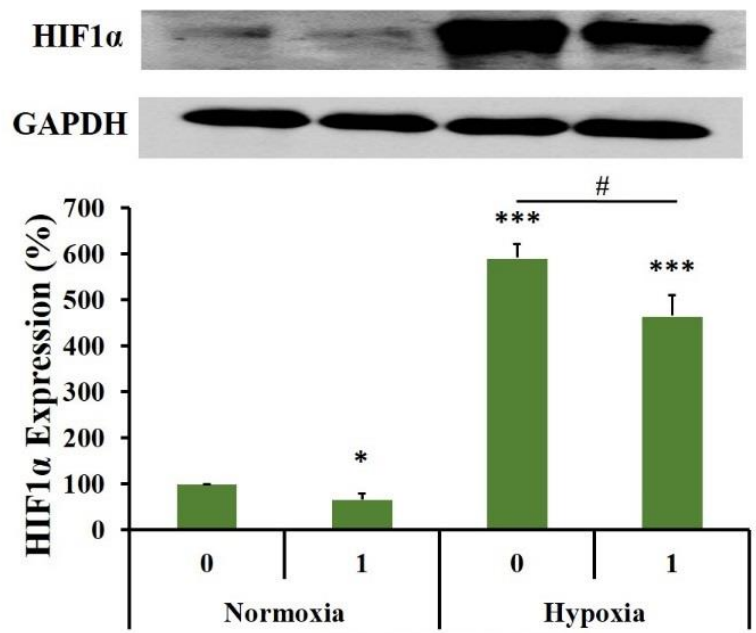

FRAX597 $(\mu \mathrm{M})$

Figure 5.3.3 Hypoxia increased expression and activity of PAK1 and FRAX597 decreased the expression of pPAK1, $\alpha$ SMA and HIF1 $\alpha$. Active form of PAK1 (pPAK1) and total PAK1 expression and $\alpha$ SMA expression were increased under hypoxia but were reduced after treatment with $1 \mu \mathrm{M}$ FRAX597 under both normoxia and hypoxia in the hPSC1 cell line (A). FRAX597 also reduced HIF1 $\alpha$ expression under both normoxia and hypoxia (B). The data (mean \pm SEM, summarised from three independent experiments) are presented as a percentage of the corresponding value for untreated normoxic cells, except for pPAK1 expression, which is presented as a percentage of the PAK1 value for untreated normoxic cells. * $\mathrm{p}<0.05$, ** $\mathrm{p}<0.01, * * * \mathrm{p}<0.001$ compared to normoxic untreated cells. $\# \mathrm{p}<0.05 ; \# \# \mathrm{p}<0.01$ as indicated in the figure.

\subsubsection{Inhibition of PAK1 by shRNA knockdown was unsuccessful in hPSC1}

PAK1 shRNA transfection was attempted in the stellate cell line, hPSC1. The transfection procedure was optimised for stellate cells with $6 \mu \mathrm{g}$ of shRNA added to the cells for 24 hours. The usual steps of colony selection and addition of antibiotics could not be undertaken, like in 


\section{CHAPTER 5}

the tumour cells (Chapter 4.2.1) as it resulted in rapid stellate cell death or senescence. As colony selection and antibiotic pressure could not be applied, the transfection was transient and could not be maintained over passages. Initial transfections resulted in decreased PAK1 protein expression in clone 2 and a substantial reduction observed in clone 3 (Fig. 5.3.4A). However, subsequent transfections were unsuccessful and failed to achieve significant reduction of PAK1 protein expression. (Fig. 5.3.4B). Hence, inadequate number of cell passages were obtained to undertake functional experiments.

A

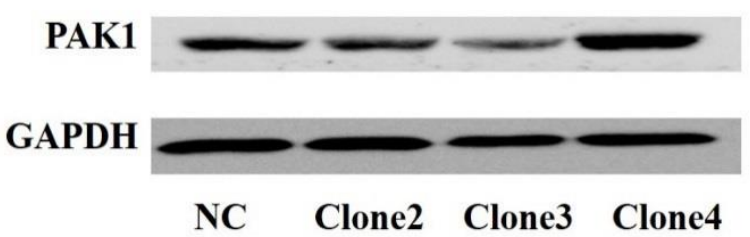

B

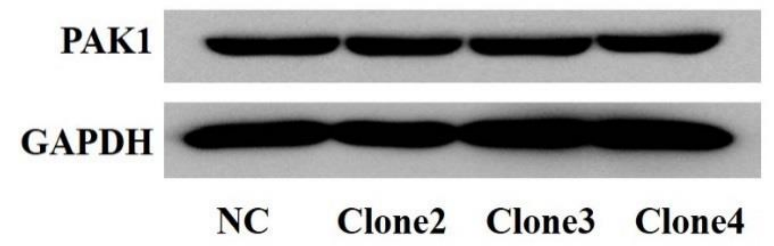

Figure 5.3.4 PAK1 knockdown (KD) of pancreatic stellate cells was unsuccessful. hPSC1 PAK1 KD cells were generated using shRNA as a transient transfection without selection or antibiotic pressure. As detected by Western blot, clone2 and clone3, resulted in reduction in PAK1 compared to negative control (NC) clones, which had been transfected with a scrambled shRNA at $6 \mu \mathrm{g}$ DNA and incubated for 24 hours (A). However, subsequent attempts could not mimic the reduction observed initially (B), so experimental data could not be confirmed or collected.

\subsubsection{PAK1 knockout quiescent murine pancreatic stellate cells had reduced proliferation}

Quiescent normal murine pancreatic stellate cells (nmPSCs) were successfully isolated from PAK1 KO and WT mice using the density gradient centrifugation method as described in Chapter 5.2.1. nmPSCs were confirmed with cell morphology, and desmin and GFAP 
immunocytochemistry staining (Fig. 5.3.5A). PAK1 KO nmPSCs had negligible amounts of PAK1 staining by immunocytochemistry and PAK1 protein expression and activity by Western blot compared to WT nmPSCs (Fig. 5.3.5B). PAK1 KO nmPSCs had significantly reduced cell growth compared to WT nmPSCs by 72 hours (Fig. 5.3.5C). These results confirm that PAK1 is involved in PSC proliferation as suggested by the anti-proliferative effects of FRAX597 found in Chapter 5.3.2.

A

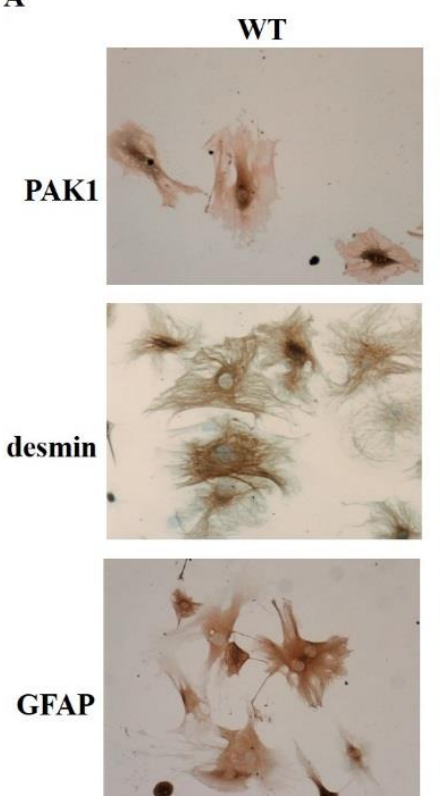

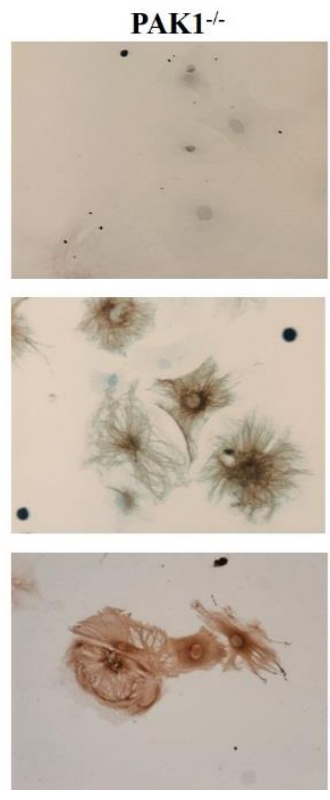

B
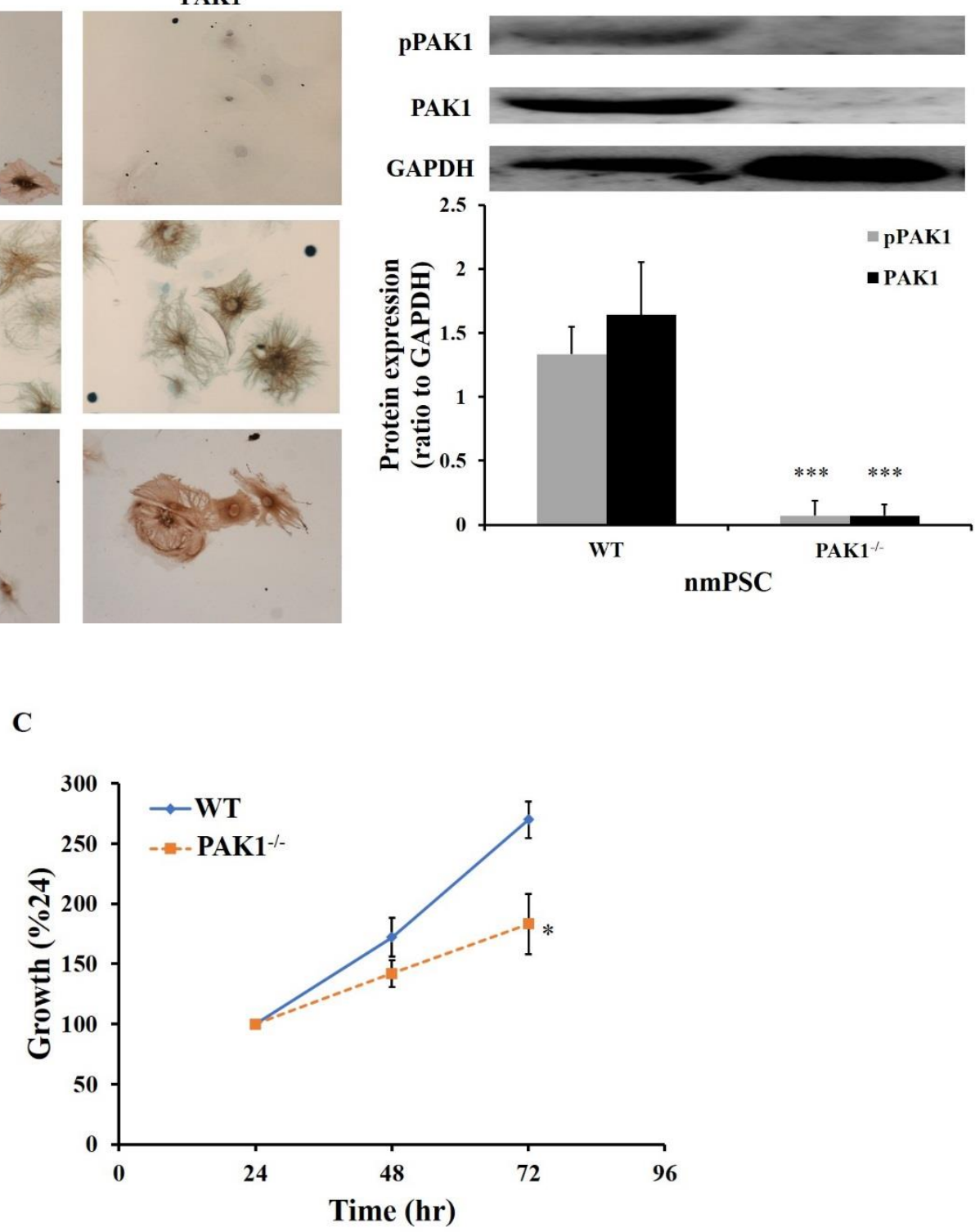
Figure 5.3.5 Isolated PSCs from PAK1 KO mice had reduced cell growth. Quiescent murine stellate cells (nmPSC) were successfully isolated from pancreases of PAK1 knockout (KO) mice and identity confirmed by desmin and GFAP (glial fibrillary acidic protein) immunocytochemistry staining (at 400x magnification) with negligible amounts of active PAK1 (pPAK1) and total PAK1 staining and protein expression (A-B). Growth of PAK1 KO nmPSCs was significantly less than WT nmPSCs at 72 hours (B). The data (mean \pm SEM, summarised from three independent experiments) are presented as a percentage to untreated cells, except for pPAK1 expression, which is presented as a percentage of the PAK1 value for untreated cells. ${ }^{*} \mathrm{p}<0.05$ compared to WT.

\subsubsection{PAK1 knockout mice had increased survival in an orthotopic murine pancreatic cancer model}

Cells of the PAK1-positive mouse pancreatic cancer cell line, Pan02, were injected into the head of the pancreas of PAK1 KO or WT mice. Survival of PAK1 KO mice was significantly greater than PAK1 WT mice, with $44 \%$ of mice surviving to the 30 -day endpoint compared to $8 \%$ of mice in the WT group (Fig. 5.3.6A). Using WT mice as a reference, PAK1 KO mice had a hazard ratio of $0.41(0.18 \pm 0.94)$, indicating a $59 \%$ survival benefit, with a p-value of 0.035 (Table 5.3.6). Examination of the tumours at the endpoint of 30 days found that the PAK1 expression and activity in the tumours from PAK1 KO mice was significantly less than in the tumours from PAK1 WT mice (Fig. 5.3.6B). PAK1 expression and activity levels in the tumours of PAK1 WT mouse were not significantly different compared to Pan02 cells. Expression of $\alpha \mathrm{SMA}$, desmin and collagen was similar between tumours from PAK1 KO and WT mice (Fig. 5.3.6C). The results demonstrated a survival improvement in depleting PAK1 
in the pancreatic cancer stroma including PSCs and suggested the ability of PAK1 in the stroma to regulate the PAK1 expression in the tumour.

A

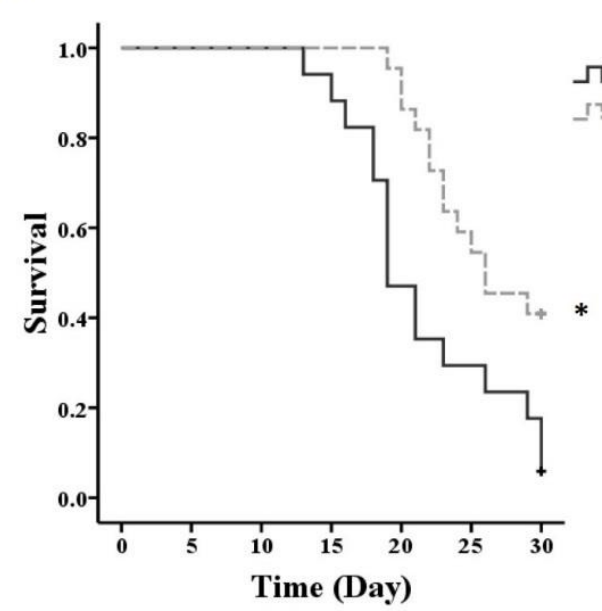

B

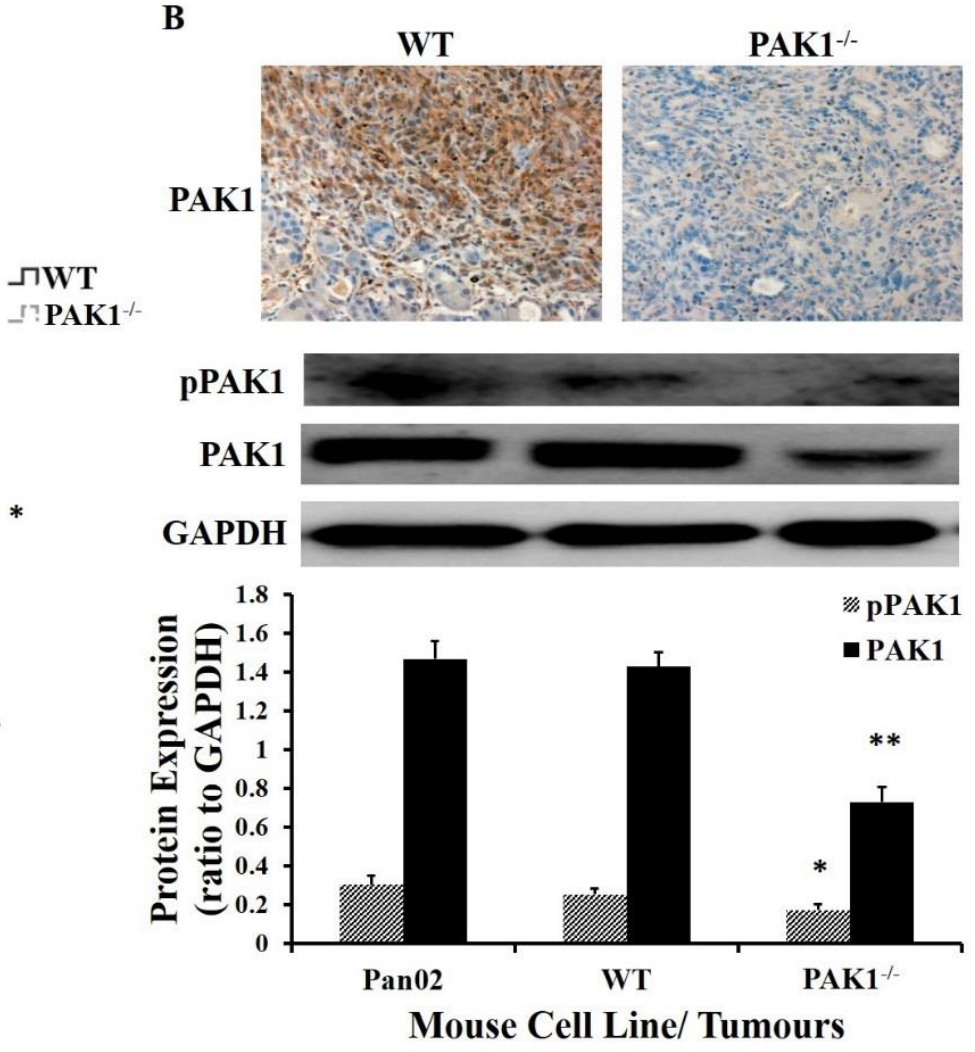

C

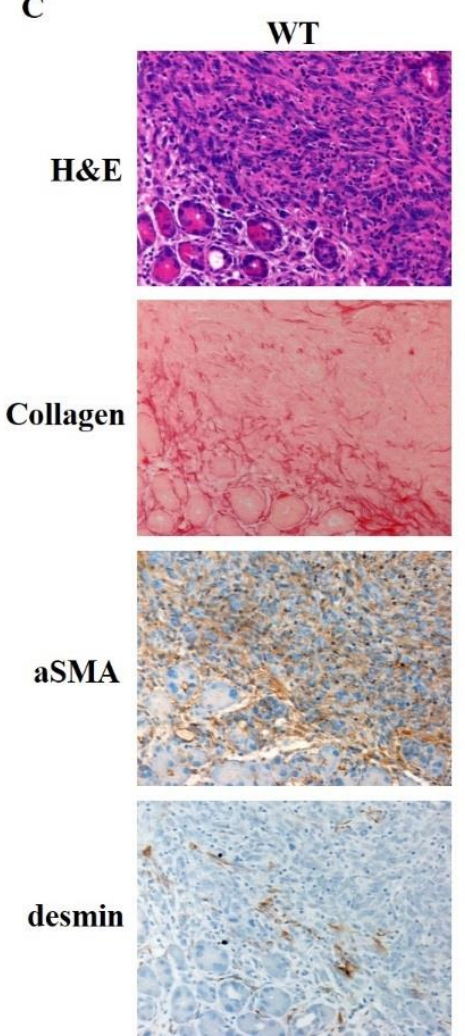

PAK1 ${ }^{-/-}$
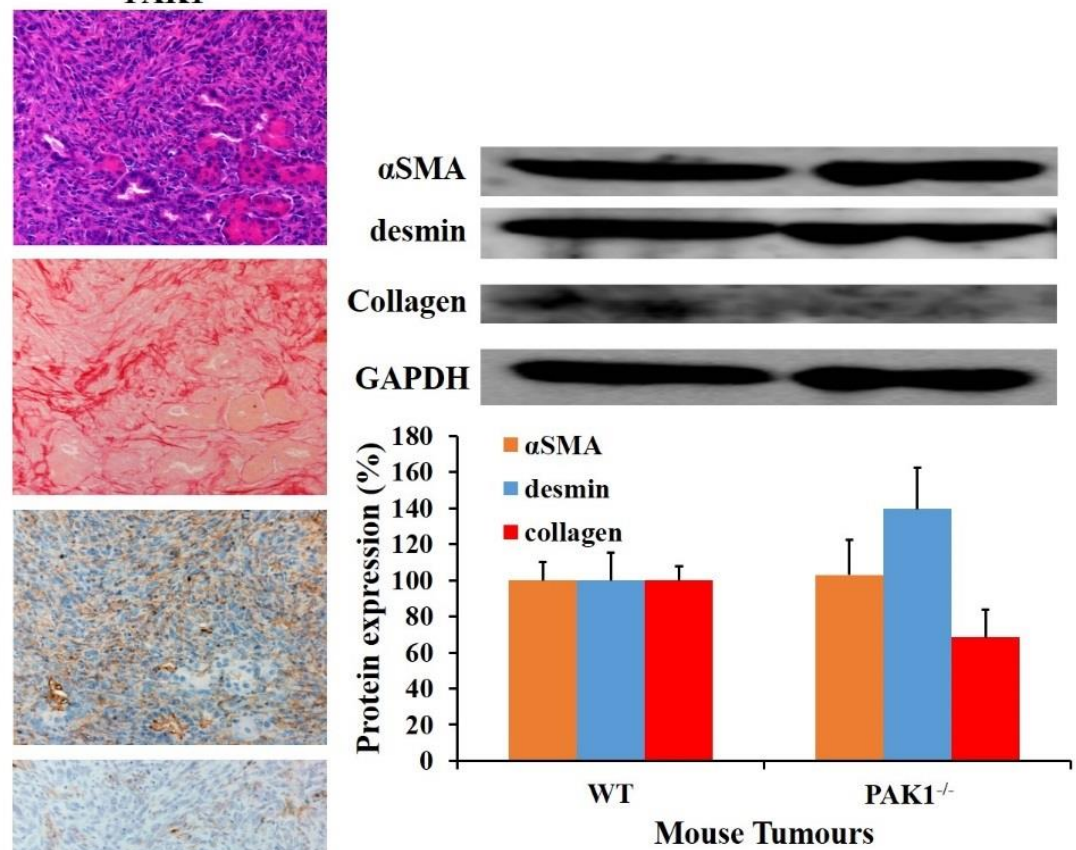
Figure 5.3.6 PAK1 KO mice had increased survival with reduced PAK1 expression in pancreatic tumours compared to PAK1 WT mice. PAK1-positive murine pancreatic cancer cell line, Pan02, were injected into the head of the pancreas of PAK1 KO (PAK1 ${ }^{-/-}$) and WT mice. Survival of PAK1 KO mice was significantly greater than the WT mice (A). Active PAK1 (pPAK1) and total PAK1 expression (ratio to GAPDH) in the tumours collected at the endpoint from PAK1 KO mice was significantly less than in tumours from WT mice based on staining and protein expression (B). PAK1 expression from Pan02 cells was also included for comparison with no difference found between the PAK1 expression from Pan02 cells and WT mice. Expression of the stellate cell and fibrotic markers, aSMA (alpha-smooth muscle actin), desmin, and collagen, was assessed in pancreatic tumours with no significant difference detected in the immunohistochemistry staining pattern (at 200x magnification) or in protein expression by Western blot $(\mathrm{C})$. The data represent mean \pm SEM. $* \mathrm{p}<0.05,{ }^{*} * \mathrm{p}<0.01$ compared to WT.

Table 5.3.6: Cox regression survival analysis of PAK1 knockout $\left(\mathrm{PAK} 1^{-/}\right)$mice and wild type (WT) mice in an orthotopic murine pancreatic cancer model.

Genotype $\quad$ Rates Ratio $\quad 95 \%$ CI p-value

WT 1.00 (ref)
$\mathrm{PAK}^{-1-}$
0.41
$0.18-0.94$
0.035

The overall statistics for the stratified Cox regression analysis were: $\chi 2(1)=4.7, p=0.031$ 
CHAPTER 5

\subsubsection{PAK1 knockout mice had transient reduction in tumour growth}

Based on the survival improvement in PAK1 KO mice and the role of PAK1 in PSC growth in vitro, the tumour growth in a mouse model was investigated. Pan02 cells were injected into the tail of the pancreas of PAK1 KO or WT mice. At the endpoint of 37 days, tumours were extracted and measured and mice scored for the presence of metastasis. There was no significant difference in tumour volume between tumours from PAK1 KO or WT mice and both groups had $75 \%$ of mice with metastasis (Fig. 5.3.7A-B). To measure the tumours growth with time, Pan02 cells were injected subcutaneously in the flank of PAK1 KO and WT mice. Tumours were measured for up to 50 days. A significant decrease was observed in the tumour volume of PAK1 KO mice from 23 days up to 42 days where the reduction was lost (Fig. 5.3.7C). These results suggested that depletion of PAK1 in the stroma does effect tumour volume but is only intermediate as the tumour growth reduction is overcome. 
A

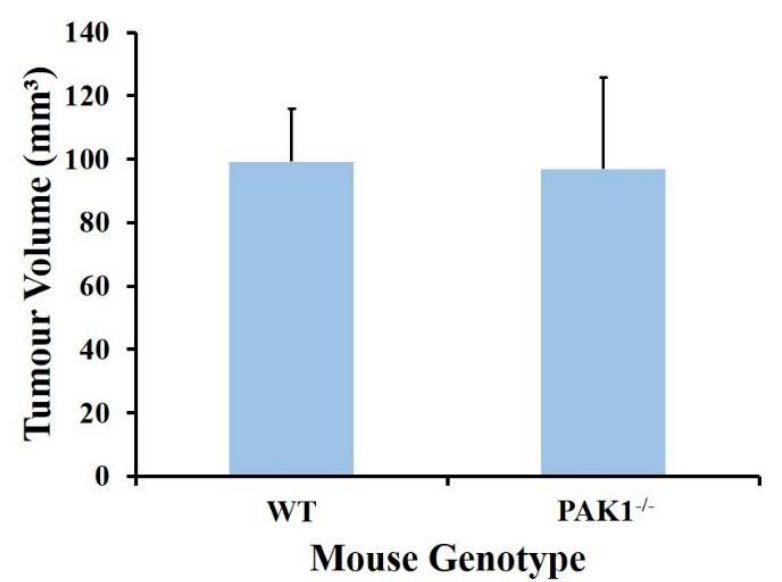

C
B

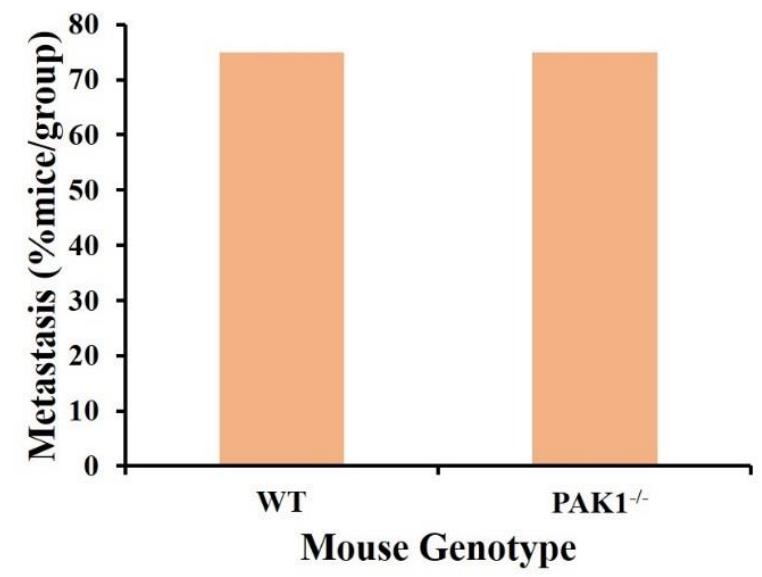

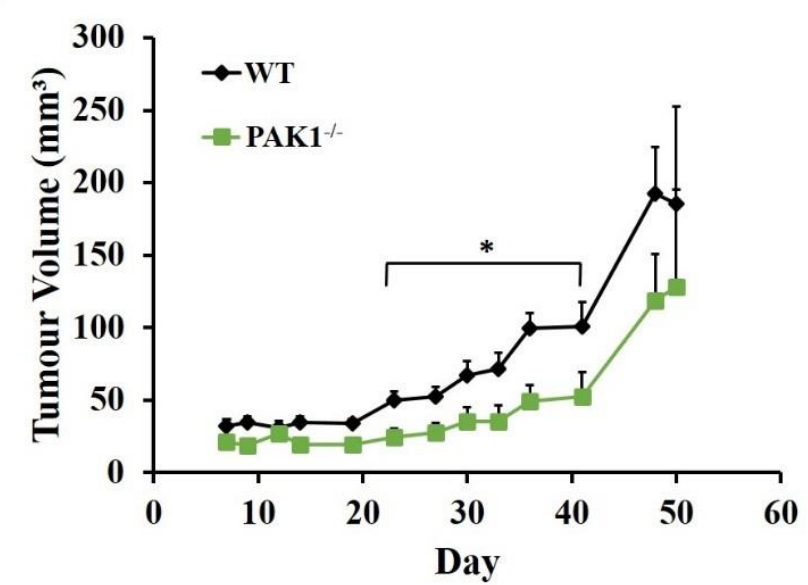

Figure 5.3.7 PAK1 KO mice reduced tumour growth but was intermittent. Pan02 cells were injected into the tail of the pancreas of PAK1 KO $\left(\mathrm{PAK}^{-/-}\right)$and WT mice. At the endpoint of 37 days, there was no difference in tumour volumes (A) or percentage of mice with metastases (B) between PAK1 KO and WT mice. Pan02 cells were injected subcutaneously of the flank of PAK1 KO and WT mice. A significant reduction in PAK1 KO tumour volumes compared to WT tumour volumes between 23 and 41 days but was not significant at the endpoint $(C)$. The data represent mean \pm SEM. $* \mathrm{p}<0.05$ compared to WT. 
CHAPTER 5

\subsubsection{Reduced PAK1 in pancreatic cancer cells by shRNA knockdown negatively regulated PSC and PSC proliferation}

To test the effect of modulation of PAK1 in tumour cells on surrounding PSCs, cells of the human pancreatic stellate cell line, hPSC1, were incubated with media conditioned either by PAK1 KD PANC-1 cells or PAK1 NC PANC-1 cells as generated in Chapter 4.3.2. PAK1 expression and activity were significantly decreased in hPSC1 cells after 48-hour incubation with conditioned medium from either KD clone (Fig. 5.3.8A). Desmin expression was also significantly decreased in hPSC1 cells incubated with conditioned medium from either KD clone, but aSMA was unchanged (Fig. 5.3.8B). Stellate cell growth was reduced when incubated with media conditioned from either $\mathrm{KD}$ clone compared to the $\mathrm{NC}$ clone (Fig. 5.3.8C) with a significant decrease in growth rate (Table 5.3.8). The results suggested that a reduction of PAK1 expression in pancreatic cancer cells suppressed the expression and activity of PAK1 in PSCs leading to reduced PSC proliferation, and hence indicated that PAK1 was important in the bidirectional signalling of PSCs and tumour cells. 


\section{CHAPTER 5}

A

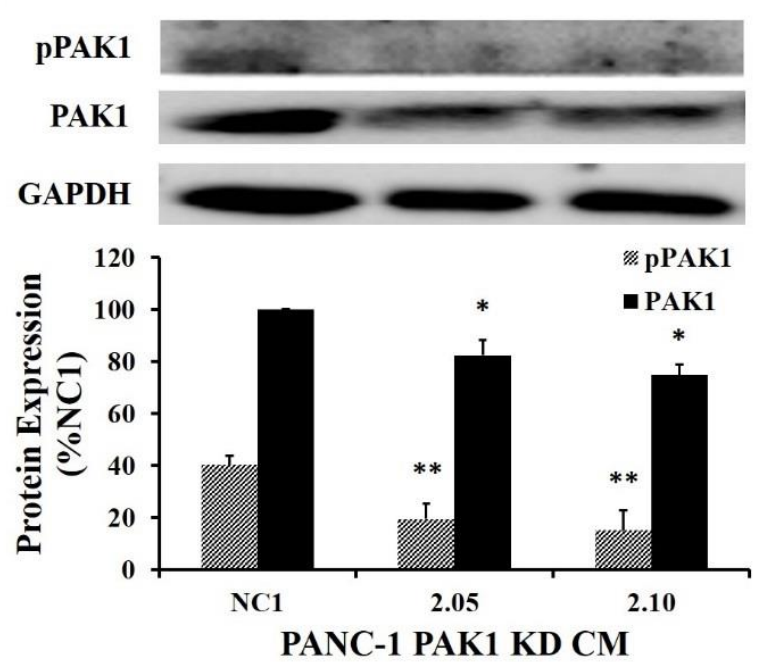

$\mathbf{B}$
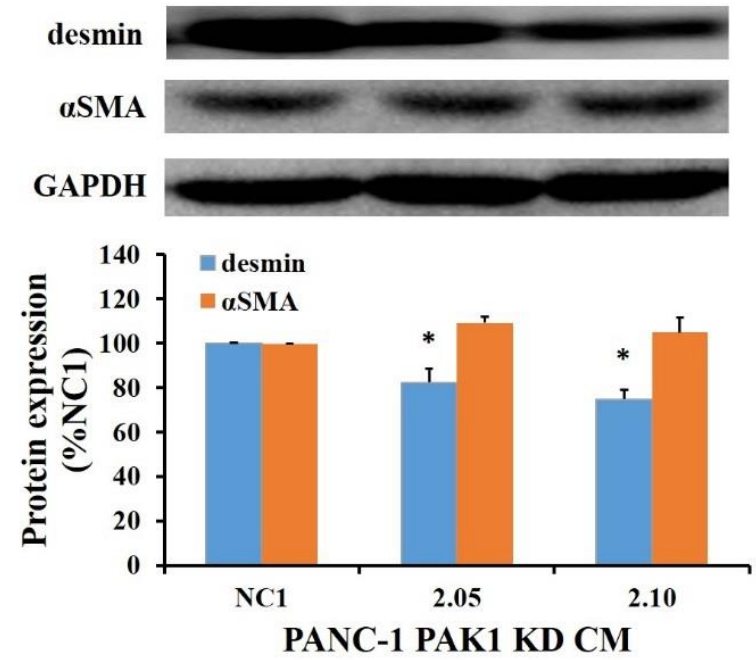

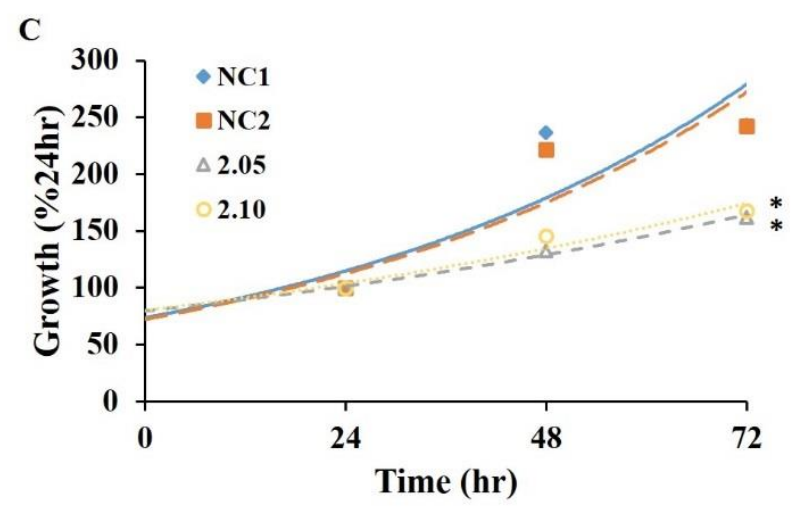

Figure 5.3.8 PAK1 knockdown in pancreatic cancer cells negatively regulated PSCs and PSC proliferation. Pancreatic stellate cells, hPSC1, incubated in PANC-1 PAK1 knockdown (KD) conditioned media (CM) for 48 hours had significantly reduced PAK1 activity and expression compared to the stellate cells incubated with the scrambled sequence, negative control (NC), cells (A). Desmin expression was also significantly reduced when incubated with PANC-1 PAK1 KD CM but $\alpha$ SMA (alpha-smooth muscle actin) expression was unchanged (B). The proliferation rate of PSC was measured by thymidine incorporation in the presence of conditioned media from PANC-1 PAK1 KD or NC cells. Significant reduction was observed in the PSC cells incubated in PANC-1 PAK1 KD CM at 48 and 72 hours compared to PSC cells incubated in PANC-1 NC CM (C). The data (mean \pm SEM, summarised from three independent experiments) are presented as a percentage to NC CM cells, except for pPAK1 


\section{CHAPTER 5}

expression, which is presented as a percentage of the PAK1 value for $\mathrm{NC} \mathrm{CM.}{ }^{*} \mathrm{p}<0.05,{ }^{* *}$ $\mathrm{p}<0.01$ compared to $\mathrm{NC}$ clone (where the higher $\mathrm{p}$ value of the two is presented if two $\mathrm{NC}$ is used).

Table 5.3.8: PAK1 knockdown (KD) conditioned media inhibited pancreatic stellate cell growth

$$
\text { PANC-1 Growth Rate (\%/hour) }
$$

\begin{tabular}{lc}
\hline $\mathrm{NC} 1$ & 1.9 \\
$\mathrm{NC} 2$ & 1.7 \\
2.05 & $1.2 *$ \\
2.10 & $1.1 *$ \\
\hline
\end{tabular}

${ }^{*} \mathrm{p}<0.05$, compared to either NC clone (where the higher $\mathrm{p}$ value of the two was used).

\subsubsection{Inhibition of PAK1 in pancreatic tumour cells by shRNA knockdown reduced PSC-} driven migration/invasion

The effect of PSCs on migration/invasion of PANC1 was measured as well as its effect on PANC-1 which had reduced PAK1 expression by shRNA knockdown, using the co-culture Transwell Boyden Chamber assay. Increasing ratio of stellate cells to tumour cells from 1:1 to 50:1 significantly increased PANC-1 cell migration/invasion (Fig. 5.3.9A). Without PSCs (ratio of 0), PANC-1 PAK1 KD cells had reduced cell migration/invasion compared to the NC clone (Fig. 5.3.9B). At the ratio of 50:1, PSCs significantly enhanced the migration/invasion 


\section{CHAPTER 5}

of PANC-1 NC cells, but not in the PAK1 KD PANC-1 cells which was not significantly increased compared the tumour migration/invasion without stellate cells. These results demonstrated that stellate cells drive tumour cell migration/invasion with higher percentage of stellate cells having a greater effect and that this is mediated through PAK1 pathways in the tumour cells.

A

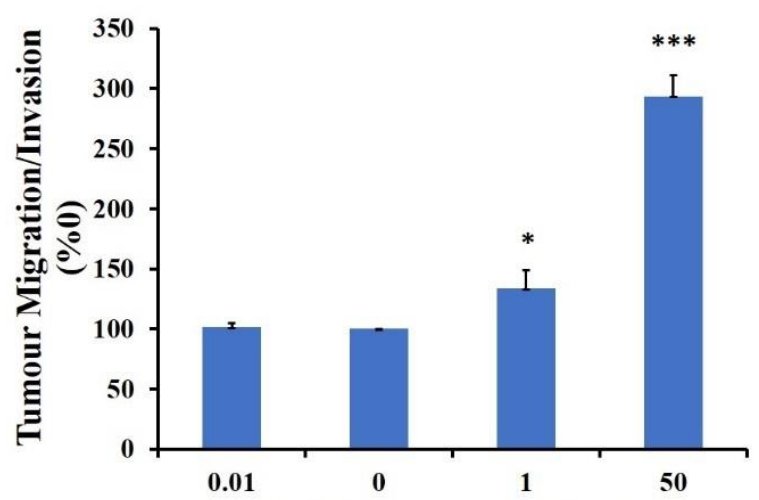

Stellate - Tumour cell ratio
B

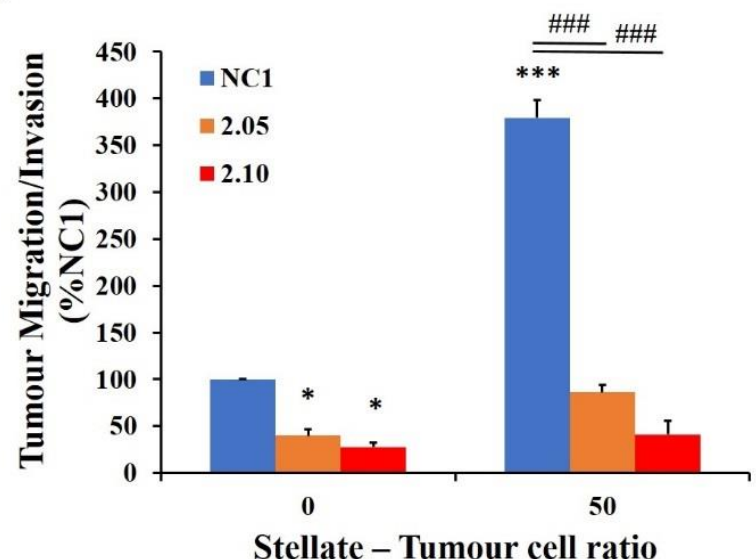

Figure 5.3.9 Tumour migration/invasion was increased in the presence of PSCs and PSCs mediated tumour migration/invasion involved PAK1 pathways. PANC-1 tumour migration/invasion was significantly increased with increasing ratios of stellate-tumour cells where tumour cell numbers was kept constant and stellate cell numbers were increased (A). In the absence of stellate cells (stellate-tumour cell ratio of 0 ), tumour cell migration/invasion was reduced in the PANC-1 PAK1 KD tumour clones: 2.05 and 2.10 (B). At a stellate-tumour ratio of 50, the migration/invasion of PANC-1 PAK1 KD cells was significantly reduced, but was not significantly different from the values without stellate cells. The data (mean \pm SEM) are expressed as a percentage of the corresponding value for the $\mathrm{NC1}$ clone at a stellate-tumour cell ratio of $0(\mathrm{~B}) .{ }^{*} \mathrm{p}<0.05, * * * \mathrm{p}<0.001$ compared to negative control or to stellate-tumour cell ratio of $0 . \# \#$ p $<0.001$ as indicated in the figure. 


\section{CHAPTER 5}

\subsection{Discussion}

This is the first study, to our knowledge, to identify a role for PAK1 in the pancreatic stroma. Expression of PAK1 was detected in the pancreatic stroma of human pancreatic cancer specimens including stroma surrounding pancreatic precursor leasions: PanINs and IPMNs (Fig. 5.3.1A). PanIN- and IPMN-associated stroma has been identified with increasing fibrosis correlated with increasing grade $(50,105)$. This observation suggests that PAK1 is involved in the stroma of pre- and established pancreatic cancers and could be a potential therapeutic target. However, fibrosis can also be due to chronic pancreatitis which is a risk factor for pancreatic cancer and often diagnosed concurrently (234). The cause of fibrosis may be an interesting area of study as PAK1 may be used as a diagnostic or prognostic marker. Nevertheless, the results demonstrate the involvement of PAK1 in the pancreatic stroma and thus, its role and effect of therapeutic targeting was investigated. Although there are many cell types within the stroma, PSCs were chosen for further study as they are primarily responsible for the extensive fibrosis observed.

Isolated human PSCs expressed PAK1. Higher $\alpha$ SMA expression (ie. more activated stellate cells) had higher PAK1 expression suggesting that PAK1 may play a role in the activation of stellate cells in pancreatic cancer. Although a major limitation of the outgrowth method which is used to isolate PSCs from pancreatic tumours is that it yields a heterogeneous population of cells comprising of different ages due to temporal differences in their growing out from the fibrotic tissue blocks (76). Thus, obtaining a pure PSC population is difficult and contamination is highly likely although none of the isolated PSCs used in this study exhibited the epithelial marker, CK19, and regular checking for quality and purity was undertaken. Due to the heterogeneous population of PSCs, conclusions from isolated PSCs should be done with caution and should be checked in pre-clinical models, hence, our use of orthotopic murine models. The exact mechanism and reason PAK1 is utilised by the stellate cells (ie. is PAK1 


\section{CHAPTER 5}

activity in stellate cells regulated by the pancreatic tumour or does the activation of stellate cells upregulate PAK1 and is necessary for its activity) is unclear and requires further elucidation. GAPDH is involved in glycolytic metabolism which has been found to be important in pancreatic cancer cells and stroma cells and its use as a housekeeping gene (Fig. 5.3.1C) presents a limitation of the results presented (16). Examination of the histopathological and patient data (data not shown) did not reveal any correlation with the level of PAK1 expression in hPSCs, however, larger numbers are needed to strengthen this conclusion. Moreover, the involvement of PAK1 in other stromal cell types such as immune cells and in neuroendocrine cells warrants further investigation $(61,108)$.

FRAX597 switched stellate cells to a more quiescent phenotype by inhibition of the activation and function of hPSCs, inhibited stellate cell proliferation by reducing the expression of cyclin D1, and promoted apoptosis (Fig. 5.3.2). FRAX597 is selective for PAK1, and also inhibits PAK2 and PAK3, but has little effect on PAK4-6 (123). FRAX597 also targets other kinases including RET, YES1, TEK, and CSF1R (123). Although the ability of FRAX597 to inhibit PAK1 was confirmed, and there is no evidence to suggest that either PAK2 or PAK3 plays any role in the pancreas $(81,115)$. Nevertheless, our results are consistent with the hypothesis that PAK1 is involved in multiple pathways including those regulating PSC activation, function, cell cycle, apoptosis and proliferation. Furthermore, the depletion of stellate cells as shown by the induction of apoptosis (Fig. 5.3.2D) and reduction in PSC function (Fig. 5.3.2B) suggests that PAK1 therapy has the potential to both deplete and reprogram stellate cells. Further studies are needed to confirm if this dual strategy of depleting and reprogramming stellate cells is viable therapeutic strategy and results in improved outcomes for pancreatic cancer patients.

Hypoxia increased PAK1 expression and activity and activated stellate cells (Fig. 5.3.3). Pancreatic tumours are known to be highly hypoxic and hypoxia has been found to induce PSC activity, migration, and activation $(51,143,182)$. The expression of HIF1 $\alpha$, a master regulator 


\section{CHAPTER 5}

of the cellular response to hypoxia, was increased under hypoxia, and as shown in Chapter 4.3.5, HIF1 $\alpha$ is downstream of PAK1 in pancreatic tumour cells. Thus, PAK1 may be induced to increase HIF1 $\alpha$ as a survival mechanism for PSCs under a hypoxic challenge. Hypoxia is known to be an activator of PSCs through stabilisation of HIF1 $\alpha$ (119) with increases in PSC activation and HIF1 $\alpha$ expression (Chapter 5.3.3). Under hypoxia, FRAX597 significantly decreased PSC activation and HIF1 $\alpha$, switching PSCs to the quiescent phenotype and reversing the effect of hypoxia-related survival. These observations further implicate PAK1 in key PSC mechanisms under hypoxia and highlight PAK1's therapeutic potential.

nmPSCs from PAK1 KO mice had impaired proliferative ability (Fig. 5.3.5). nmPSCs were successfully isolated from PAK1 KO mice and confirmed to be 'normal' PSCs with comparable cell morphology and desmin and GFAP staining compared to nmPSCs isolated from wildtype mice. The impaired proliferation of nmPSCs from PAK1 KO mice confirmed the role of PAK1 in PSC proliferation as also found when treated with the group 1 PAK inhibitor, FRAX597. Further elucidation of the role of PAK1 utilising the PAK1 KO nmPSCs is needed but illustrates the successful isolation of nmPSCs to investigate PAK1.

Decreased activity and expression of PAK1 in the pancreatic orthotopic tumours in PAK1 KO mouse resulted in increased mouse survival (Fig. 5.3.6). The pancreatic tumours grown in PAK1 KO mice had reduced PAK1 staining although the tumour cells were originally PAK1 positive at induction. This observation suggests that the stroma can regulate PAK1 expression in the tumours, possibly through the involvement of PAK1 negative regulators. Although the mechanism that resulted in the downregulation of tumoural PAK1 is unclear, the observed reduction does highlight the importance of stromal PAK1, the involvement of PAK1 in the complex interaction between PSCs and the tumour, and the potential therapeutic benefit of targeting PAK1 in the stroma to increase pancreatic cancer survival. However, a possible limitation of this murine survival study is the use of C57B1/6 mice as WT mice which may 


\section{CHAPTER 5}

have a different genetic background compared to the PAK1 KO mice. For future experiments, PAK1 KO and PAK1 WT mice should be bred from PAK1 heterozygous KO mice to ensure similar genetic background. In conclusion, genetically engineered mice that can decrease PAK1 in the stroma or more specifically in the PSCs are needed to confirm these results.

A decrease in tumour volume was not observed in an orthotopic murine model in PAK1 KO mice at the endpoint (Fig. 5.3.7A). Based on the results that PAK1 is involved in PSC activation, activity and proliferation, and the survival improvement resulting in a decrease in PAK1 expression in the tumours, it would be expected to observe some difference in tumour volume in the orthotopic tumours. The implantation location may be a factor as human resected pancreatic head compared to pancreatic tail tumours have different molecular characteristics, although confirmatory studies are needed (127). Real-time monitoring of mouse tumours and real-time imaging of the associated fibrosis is needed as it would provide a better contextual understanding. As an alternative, tumour growth was measured in subcutaneous flank tumours (Fig. 5.3.7C). Although there is an absence of certain stromal cells such as stellate cells, a transient decrease in tumour growth was noted and suggests the involvement of PAK1 in the immune response or other stromal components on pancreatic tumour growth. The results require additional investigations but highlight the dynamic and complex nature and the need for contextual understanding to guide therapeutic strategies.

PAK1 plays an important role in the interaction between PSCs and tumour cells. PAK1 activity and expression in stellate cells was reduced resulting in a decrease in stellate cell growth (Fig. 5.3.8) when PSCs were cultured with the media conditioned by tumour cells in which PAK1 had been knocked down by transfection with shRNA. The decrease in cell proliferation suggests possible PAK1-dependent pathways that control stellate cell growth and confirms the importance of PAK1 in stellate cell growth as shown in the FRAX597 treatment and PAK1 KO nmPSCs. The decrease in PAK1 expression suggests that PAK1-dependent pathways in 


\section{CHAPTER 5}

the tumour control the secretion of factors that regulate the expression of PAK1 in the stellate cells. Together with the in vivo results, this observation suggests that targeting PAK1 expression in either PSCs or tumour cells results in downregulation of PAK1 in the other cell type. Knockdown of tumoural PAK1 also resulted in a decrease in stellate cell function. Although this result shows that PAK1 in the tumours is responsible for the secretion of factors which regulate stellate function, PAK1 is unlikely to be the main regulator as only a modest decrease was observed and many other pathways, which have been well studied, are also involved (144). Although this study only examines the unidirectional interaction, it does implicate the involvement of PAK1 pathways in the tumour in controlling the secretion of factors influencing PAK1 regulation in the stellate cells, their function and proliferation.

PSC-driven migration and invasion of tumours are PAK1-dependent (Fig. 5.3.9). Increasing stellate-tumour cell ratios resulted in increased migration and invasion of tumour cells with a 3 -fold increase at a ratio of 50. This has been well studied with many studies observing an increase in proliferation and metastasis when PSC are co-cultured or implanted with tumour cells in mice, however a single ratio of stellate-tumour cells are used $(74,110,224,238)$. In the absence of PSCs, PAK1 KD tumour cells had reduced migration/invasion. When incubated with PSCs, migration/invasion of PAK1 KD tumour cells was further impaired, compared to controls. This result suggests that PSCs secrete factors that regulate the migration/invasion of tumour cells and must involve PAK1 pathways in the tumour. Hence patients with tumours with low PAK1 expression may benefit from stromal depletion therapy or stellate cell-targeted therapy as tumours have reduced metastatic ability regardless of the presence of PSCs, however, clinical validation is needed. 


\section{CHAPTER 5}

\subsection{Conclusion}

PAK1 is expressed in the stroma of pancreatic cancer specimens, as well as in PSCs isolated from human pancreatic cancer patients. Hypoxia increased PAK1 activity and expression in the human pancreatic stellate cell line, hPSC1. A group 1 PAK inhibitor, FRAX597, inhibited proliferation through reducing the activity of PAK1 and cyclin D1 protein expression. FRAX597 also increased apoptosis, and reduced PSC activation, and HIF1 $\alpha$ expression. PAK1 was found to play a role in the complex interaction between PSCs and tumour cells. Depletion of stromal PAK1 using PAK1 KO mice increased mouse survival through the reduction of PAK1 activity and expression in pancreatic tumours generated by inoculation of murine Pan02 cells, which were originally PAK1 positive. This result suggests that an indirect strategy to target the pancreatic tumours by modulation of the stroma would be effective, however further validation is needed. The regulation of PAK1 between PSCs and tumour cells was found to be bidirectional with the regulation of PAK1 in PSCs dependent on the PAK1 expression in the tumour cells using conditioned media from PAK1 KD tumour cells. PSC-driven migration and invasion of tumour cells was found to utilise PAK1 pathways since PAK1 KD tumour cells had impaired migration/invasion in the presence of PSCs and illustrated a case where stromal depletion may be beneficial and should not be disregarded as a therapy in selected cases. In conclusion, PAK1-directed therapy has the potential to not only disrupt the pancreatic cancer stroma, by depleting and reprogramming the PSCs resulting in reduced fibrosis, but also to reduce pancreatic tumour metastasis and hence increase survival in patients with pancreatic cancer. 
CHAPTER 6

Chapter 6: General Discussion and Future

\author{
Directions
}




\section{CHAPTER 6}

This general discussion reviews the broad findings and implications of this project and outlines the limitations of this project, challenges in investigating PAK1 in pancreatic cancer, and future directions.

\subsection{PAK1 plays an important role in pancreatic cancer}

Pancreatic cancer is a dismal disease with limited therapeutics available. In the hopes of improving pancreatic cancer patient outcomes, a more comprehensive understanding is required so therapeutics can be used in an effective manner. PAKs are well studied, known to have a role in carcinogenesis and act downstream of the KRAS mutation which have a high prevalence in pancreatic cancer. Although two isoforms of PAKs, PAK1 and PAK4, have been identified as either being hyper-activated or upregulated and both isoforms were confirmed to be present in the pancreatic cancer cell lines (Fig. 3.3.3), the main focus of this thesis was elucidating the role of PAK1.

PAK1 expression was found to be upregulated in human pancreatic cancer compared to the progenitor cells: pancreatic acinar and ductal cells, and in pancreatic cancer cell lines compared to immortalised normal pancreatic epithelial cells (Fig. 4.3.1). The upregulated expression suggested that PAK1 played a role in pancreatic carcinogenesis and was explored.

First, using a natural product, glaucarubinone, which had been found to inhibit, in part, through PAK1 signalling, was investigated. Treatment with glaucarubinone reduced pancreatic cancer proliferation (Fig. 3.3.1) and migration/invasion (Fig. 3.3.2). As two PAK isoforms had been identified in pancreatic cancer, both were examined under the treatment of glaucarubinone, although evidence for glaucarubinone acting on PAK4 was limited. It was found that glaurcaurbinone, in part, acted on pathways involving both PAK1 and PAK4 (Fig. 3.3.5 \& 3.3.7). At time of publishing these results, it was the first study to show a role of PAKs in pancreatic cancer and the possible clinical benefit of using an inhibitor to inhibit PAKs. 


\section{CHAPTER 6}

To further investigate the role of PAK1, inhibition of PAK1 by knockdown transfection and a group 1 PAK inhibitor, FRAX597, was used. Using PAK1 KD cells, PAK1 was found to be involved in cell proliferation, survival (Fig. 4.3.2) and clonogenic growth (Fig. 4.3.4) of human pancreatic cancer cell lines and mediated AKT and HIF1 $\alpha$ pathways (Fig. 4.3.5). Furthermore, inhibition of PAK1 sensitised pancreatic cancer cells to gemcitabine (Fig. 4.3.3). Using the group 1 PAK inhibitor, FRAX597, the role of PAK1 was further confirmed to be implicated in cell proliferation, migration/invasion, and survival (Fig. 4.3.7). FRAX597 treated cells were also sensitised to gemcitabine (Fig. 4.3.8) and in addition, to 5-FU (Fig. 4.3.9), suggestive of a general sensitisation to chemotherapies. These results indicated the varied roles that PAK1 was involved in pancreatic cancer and outlined the benefit of inhibiting PAK1 function on pancreatic cancer outcomes using a PAK1 inhibitor in combination with standard chemotherapy.

PAK1 was also found to play a role in the pancreatic stroma. PAK1 was expressed in the stroma of pancreatic cancer specimens, as well as in PSCs isolated from pancreatic cancer patients (Fig. 5.3.1). PAK1 was found to be involved in PSC activation, function, proliferation and apoptosis (Fig. 5.3.2) using the group 1 PAK1 inhibitor, FRAX597, and the role of PAK1 in proliferation was confirmed using PSCs isolated from PAK1 KO mice (Fig. 5.3.5). PAK1 was found to play a role in the complex interaction between PSCs and tumour cells. Depletion of stromal PAK1, including PSCs using PAK1 KO mice resulted in the reduction of PAK1 activity and expression in pancreatic tumours which were originally PAK1 positive (Fig. 5.3.6). The regulation of PAK1 between PSCs and tumour cells was found to be bidirectional with the regulation of PAK1 in PSC dependent on the PAK1 expression in the tumour cells using conditioned media from PAK1 KD tumour cells (Fig. 5.3.8). Furthermore, PAK1 was implicated in PSC-driven tumour migration/invasion using co-culture experiments (Fig. 5.3.9). This was the first study to investigate the role of PAK1 in the pancreatic cancer stroma and 


\section{CHAPTER 6}

PSCs and found that PAK1 was involved in several important cellular process and interactions with tumour cells, helping unravel the complexity of this disease and highlighting aspects for therapeutic intervention.

\subsection{PAK1 inhibition combined with gemcitabine improved survival and decrease tumour growth in murine models}

The finding that PAK1 was involved in a number of signalling pathways makes it a likely therapeutic target for pancreatic cancer. PAK inhibitors: glacuarubinone and FRAX597, combined with the chemotherapy, gemcitabine, was used to examine the effect of targeting and inhibiting PAK1 on pancreatic cancer patient outcomes. Using orthotopic murine pancreatic cancer models, the combination of glaucarubinone and gemcitabine treatment significantly improved murine survival compared to gemcitabine treatment alone (Fig. 3.3.8). Also, FRAX597 combined with gemcitabine inhibited pancreatic cancer growth, metastasis, and improved murine survival (Fig. 4.3.11). In both studies, there was no apparent increase in toxicity compared to the gemcitabine treatment alone. This illustrated that inhibitors that target PAK1, combined with gemcitabine had the potential to inhibit pancreatic cancer growth and improve patient survival.

Further investigation into the orthotopic tumours treated with the FRAX597 inhibitor revealed a decrease in collagen deposition, prompting the investigation in Chapter 5. The effect of depleting stromal PAK1 was examined using PAK1 KO mice with PAK1 KO mice found to have an increase in pancreatic cancer survival and a transient decrease in tumour growth (Fig. 5.3.6). Furthermore, the tumours from the PAK1 KO mice had reduced PAK1 expression, highlighting a possible indirect mechanism for targeting PAK1 in the tumours. Although this may be an artificial system, as the stroma of these mice did not have PAK1, a clinical model where stromal PAK1 deletion occurs on established tumours is needed but it does illustrate the 


\section{CHAPTER 6}

potential of targeting stromal PAK1. PAK1 inhibition of PSCs results in both stellate cell depletion and inactivation of stellate cells which has not been evaluated in the clinical setting but combined with other therapies, has the ability to increase drug delivery and improve the survival of pancreatic cancer patients.

\subsection{Limitations and future directions}

As with all pre-clinical experiments, there are a number of limitations and caution is needed before translating the conclusions directly. The heterogeneity of pancreatic cancer adds an extra dimension of difficulty of finding the right treatment to the right patient. With the increased availability of genetic sequencing techniques, two recent studies have identified 4 subclasses of pancreatic cancer patients which may assistin identifying certain patients with similar cancer biology who would most benefit from certain types of therapy $(153,226)$. PAKs were chosen due to the high prevalence of the KRAS mutation in pancreatic cancer but as seen in the staining of pancreatic cancer specimens, a range of PAK1 staining is observed in both tumour (Fig. 4.3.1) and stroma (Fig. 5.3.1) components. Furthermore, the identification of PAK1 expression in $K R A S$-wildtype cell lines, BxPC3 and Pan02 (Fig. 4.3.1B) suggests that PAK1 expression may not necessarily be dependent on its KRAS mutational status. Further analysis and larger patient cohorts is needed to determine the PAK1 expression in the subtypes that have been recently identified from genomic sequencing which may determine the exact mechanism of upregulation of PAK1 and identify those patients who would most benefit from PAK1 therapy.

Thorough investigation of pancreatic carcinogenic functions that involve PAK1 has been examined in this thesis. However, the exact mechanisms and elucidation of other pathways that involve PAK1, upstream and downstream, is needed. Together with other study's findings, PAK1-mediated downstream signalling have found the involvement of AKT, HIF1 $\alpha, \mathrm{NF}$ B and HGF/MET pathways $(102,259)$. Investigating downstream signalling pathways is difficult 


\section{CHAPTER 6}

as the heterogeneity of pancreatic cancer may lead to differences in PAK1-mediated pathways from one patient to another as observed with the difference in signalling protein expression in PAK1 KD cells of PANC-1 and MiaPaCa-2 (Fig. 4.3.5G-H). Whole proteomic analysis is required to further investigate PAK1-mediated signalling and may elucidate other key signalling pathways downstream of PAK1.

Our results demonstrate the importance of PAK1 signalling in pancreatic cancer and the possible benefit of using a PAK1 targeted therapy in combination with other therapies to improve pancreatic cancer patient outcomes. However, a specific and effective PAK1 inhibitor is yet to be discovered with no PAK1 inhibitors currently in the clinical setting. The PAK inhibitors used in this thesis: glaucarubinone and FRAX597, have other targets but they were both confirmed to decrease PAK1 activity (Fig. 3.3.5 \& 4.3.10). Although FRAX597 was developed to target group 1 PAKs, as an ATP-competitive inhibitor, it was also found to target other kinases: RET, YES1, TEK, and CSF1R, and their possible involvement cannot be disregarded in the FRAX597-treated results (123). Thus, investigation of the regulatory signals of PAK1 is needed with the hope that a more comprehensive understanding of the negative regulators of PAK1 may assist in PAK1 drug discovery and development. It would also give insight into the mechanisms of how PAK1 is upregulated in pancreatic cancer. Further understanding into the regulatory mechanisms of PAK1 is needed to aid the discovery of an effective and specific PAK1 inhibitor for clinical use.

The finding that glaucarubinone inhibited PAK4, as well as PAK1, resulted in inhibition of pancreatic cancer growth (Fig. 3.3.6) and improvement in survival (Fig. 3.3.8) requires further investigation into the role of PAK4. Since our study, other groups have found that PAK4 is overexpressed in pancreatic tumours compared to normal tissues and is a predictive marker of gemcitabine sensitivity $(158,210)$. There is a need to elucidate the role of PAK4 in pancreatic 


\section{CHAPTER 6}

cancer as the similarity in isoform structure may act as a counter-regulatory mechanism when one of the PAK isoform is inhibited. Furthermore, it is likely, that a PAK1 inhibitor may have some inhibitory effect on PAK4 and vice versa. Thus, it is important to explore PAK4's role in pancreatic cancer and examine the effect of PAK4 inhibitors in pancreatic cancer.

The utilisation of murine models to assess PAK1 function as a preclinical model confirms our in vitro data and provides a dynamic system to explore the role of PAK1 in pancreatic cancer. Mouse models of pancreatic cancer has advanced significantly over the past few years with the generation of orthotopic murine models and genetically engineered mouse models $(68,85)$. The findings of our results should be confirmed with genetically engineered mouse models such as the KPC (Kras $\left.{ }^{\mathrm{G} 12 \mathrm{D}} ; \operatorname{Trp} 53^{\mathrm{R} 172 \mathrm{H}} ; \mathrm{Pdx} 1-\mathrm{Cre}\right)$ mice model where pancreatic tumours spontaneously arise and metastasis occur in a similar fashion as the human tumours. Although, these models are significantly longer than the orthotopic models used in this thesis, they would allow for the investigation of PAK1 on metastasis and evaluation of PAK1 inhibitors on the development of pancreatic precursor lesions as a preventative treatment. However, there are many differences in the pancreas of mice compared to human such as structure, biology and morphology, and raises a major challenge in successfully translating findings from mice models (42). Recently, the development of organoids from human pancreatic cancer samples attempts to bridge this gap by culturing tumour cells and their associated microenvironment together where assessment of the genetic, molecular and proteomic changes and inhibitor testing can be undertaken (17). The system's ability to mimic the inter- and intra-heterogeneity of pancreatic cancer makes it a useful model as the field moves towards personalised medicine, however, the findings may only be applicable for that patient and the viability and costeffectiveness of such a strategy is unmaintainable. Nevertheless, organoids and genetically modified murine models provide a translational platform to test PAK1 inhibitors and identify 


\section{CHAPTER 6}

patients who would benefit from PAK1 therapy and our results should be confirmed using these systems before clinical trials.

The growing evidence that the stroma is an important factor in pancreatic cancer is a challenge in any therapeutic intervention for pancreatic cancer for both drug delivery and drug resistance. As highlighted in the results presented, the stroma interaction with the tumour cells is dynamic and complex with PAK1 regulation found to be bidirectional (Fig. 5.3.6B \& 5.3.8A). Any new therapy for pancreatic cancer should be examined for effects to the microenvironment such as with the FRAX597 treatment (Fig. 4.3.13 \& Chapter 5) to ensure a better understanding of the therapeutic effects. Although the main consensus in the field currently is to target the stroma to a quiescent state rather than depleting it, our understanding of the role of the stroma at the different disease stages and sites is lacking and both therapeutic strategies may have a place in pancreatic therapy or even one that does both in the case of PAK1 therapy (164). Furthermore, the heterogeneity of the stroma of pancreatic cancer and how PAK1 may be involved in those stromal subclasses requires further investigation as those presented in this thesis may not apply to all patients $(60,153)$. Human pancreatic cancer organoids may assist in providing a more comprehensive study into their interaction but still requires further validation. The dynamic and complex nature of the pancreatic cancer stroma makes studying them difficult and caution is needed in translating their results.

The stroma is a dynamic entity and may be involved in resistant mechanisms. The stroma's role in drug resistance is well studied with a recent study identifying fibroblasts playing a role in gemcitabine resistance in tumour cells and the stroma acting as a barrier for drug delivery (59). PAK1 acquired resistance from PAK1 therapy is unknown and has not been explored. However, there was a transient growth reduction in depleted stromal PAK1 pancreatic cancer mice (Fig. 5.3.7), indicating that the stromal-tumour interactions can overcome the depletion effect and possibly rely on other mechanisms. Identifying these mechanisms may result in other 


\section{CHAPTER 6}

therapeutic targets for combinational treatment with PAK1 therapy to prevent resistance. Furthermore, PSCs were the focus of our study, but other stromal cells should be investigated if PAK1 plays a role. Although, PAK1 has been implicated in chemotherapeutic resistance (Fig. 4.3.3) in the tumour cells, its role in the resistance to PAK1 therapy in the context of the pancreatic stroma is still to be elucidated and requires real-time analysis of their interaction.

The results presented support the use of PAK1 inhibitors for the clinical treatment of pancreatic cancer patients. However, confirmatory clinical trials are needed. Recruitment of patients may be difficult so early trials in those that had pancreatic cancer resections may provide an avenue for evaluation in the neo-adjuvant and adjuvant setting. Furthermore, our preclinical data, mouse model experiments, have been designed to start treatment early for the fullest effect, which would be similar to patients that have residual tumour cells following resection. If the results are positive, further trials with patients who have locally advanced, metastatic pancreatic cancer may be undertaken in a blinded, randomised manner. However, based on our staining of pancreatic cancer samples (Fig. 4.3.1A \& 5.3.1A), there is a range of PAK1 staining in the tumoural and stromal regions from sample to sample. Thus, some patients may have higher PAK1 staining in the tumoural or stromal region and these patients may respond better to PAK1-directed therapy. A large micro-array is currently being collated from patients who have undergone pancreatic cancer resections at the Austin Hospital. The results from this micro-array would be able to observe the extent of PAK1 expressions in the tumoural and stromal region and the estimated percentage of patients with high PAK1 in the tumour or stroma. Also evaluation of PAK1-specific inhibitors, like FRAX597, versus non-specific PAK1 inhibitors, like glaucarubinone, is needed and may direct clinical trials over the type of inhibitor that may more likely succeed in improving pancreatic cancer patient outcomes. 


\section{CHAPTER 6}

\subsection{Conclusion}

The results presented demonstrate the upregulation and expression of PAK1 in the pancreatic tumour and stroma, specifically the PSCs. PAK1 was implicated in pancreatic cancer cell proliferation, survival, migration/invasion, clonogenic growth and sensitised cells to gemcitabine chemotherapy using PAK1 knockdown cells and PAK1 inhibitors: glaucarubinone and FRAX597. Glaucarubinone or FRAX597 combined with gemcitabine inhibited pancreatic cancer growth, metastasis, and improved survival in vivo, supporting the use of PAK1 inhibitors for the treatment of pancreatic cancer.

PAK1 was involved in PSC proliferation, apoptosis, and their activation. PAK1 was implicated in the interaction between PSCs and tumour cells where PAK1 regulation in PSCs were dependent on PAK1 expression in the tumour cells and PSC-driven migration/invasion signalled through PAK1-mediated pathways in the tumour cells. Targeting stromal PAK1 increased mouse survival, using PAK1 knockout mice, and stromal PAK1 regulated PAK1 expression in the pancreatic tumours. The results suggest the possibility to indirectly target pancreatic tumours by modulation of the stroma. Although clinical validation is required, PAK1-directed therapy has the potential to target both the stroma and tumour by disrupting and reprogramming the pancreatic cancer stroma, increasing drug delivery to the tumour where PAK1-direct therapy can then reduce pancreatic tumour growth and metastasis and hence increase survival in patients with pancreatic cancer. 


\section{REFERENCES}

\section{References}

1. This Is The First Study Using Escalating Doses Of PF-03758309, An Oral Compound, In Patients With Advanced Solid Tumors. ClinicalTrials.gov: U.S. National Institutes of Health, 2013.

2. Abo A, Qu J, Cammarano MS, Dan C, Fritsch A, Baud V, Belisle B, and Minden A. PAK4, a novel effector for $\mathrm{Cdc} 42 \mathrm{Hs}$, is implicated in the reorganization of the actin cytoskeleton and in the formation of filopodia. The EMBO journal 17: 6527-6540, 1998.

3. Abrol R, Edderkaoui M, Goddard WA, 3rd, and Pandol SJ. Molecular basis for the interplay of apoptosis and proliferation mediated by Bcl-xL:Bim interactions in pancreatic cancer cells. Biochemical and biophysical research communications 422: 596-601, 2012.

4. Aghdassi A, Phillips P, Dudeja V, Dhaulakhandi D, Sharif R, Dawra R, Lerch MM, and Saluja A. Heat shock protein 70 increases tumorigenicity and inhibits apoptosis in pancreatic adenocarcinoma. Cancer research 67: 616-625, 2007.

5. AIHW. Australian Cancer Incidence and Mortality (ACIM) books: pancreatic cancer. Canberra, 2016.

6. Alahari SK, Reddig PJ, and Juliano RL. The integrin-binding protein Nischarin regulates cell migration by inhibiting PAK. The EMBO journal 23: 2777-2788, 2004.

7. Apte MV, Haber PS, Applegate TL, Norton ID, McCaughan GW, Korsten MA, Pirola RC, and Wilson JS. Periacinar stellate shaped cells in rat pancreas: identification, isolation, and culture. Gut 43: 128-133, 1998.

8. Arias-Romero LE, Villamar-Cruz O, Pacheco A, Kosoff R, Huang M, Muthuswamy SK, and Chernoff J. A Rac-Pak signaling pathway is essential for ErbB2mediated transformation of human breast epithelial cancer cells. Oncogene 29: 5839-5849, 2010 .

9. Azevedo-Pouly AC, Sutaria DS, Jiang J, Elgamal OA, Amari F, Allard D, Grippo PJ, Coppola V, and Schmittgen TD. miR-216 and miR-217 expression is reduced in transgenic mouse models of pancreatic adenocarcinoma, knockout of miR-216/miR-217 host gene is embryonic lethal. Functional \& integrative genomics, 2016.

10. Bachem MG, Schneider E, Gross H, Weidenbach H, Schmid RM, Menke A, Siech M, Beger H, Grunert A, and Adler G. Identification, culture, and characterization of pancreatic stellate cells in rats and humans. Gastroenterology 115: 421-432, 1998. 


\section{REFERENCES}

11. Bailey JM and Leach SD. Signaling pathways mediating epithelial- mesenchymal crosstalk in pancreatic cancer: Hedgehog, Notch and TGFbeta. In: Pancreatic Cancer and Tumor Microenvironment, edited by Grippo PJ and Munshi HG. Trivandrum (India), 2012.

12. Balasenthil S, Sahin AA, Barnes CJ, Wang RA, Pestell RG, Vadlamudi RK, and Kumar R. p21-activated kinase-1 signaling mediates cyclin D1 expression in mammary epithelial and cancer cells. The Journal of biological chemistry 279: 1422-1428, 2004.

13. Baskaran Y, Ng YW, Selamat W, Ling FT, and Manser E. Group I and II mammalian PAKs have different modes of activation by Cdc42. EMBO reports 13: 653-659, 2012.

14. Ben-Neriah Y and Karin M. Inflammation meets cancer, with NF-kappaB as the matchmaker. Nature immunology 12: 715-723, 2011.

15. Beutler JA, Kang MI, Robert F, Clement JA, PelletierJ, Colburn NH, McKee TC, Goncharova E, McMahon JB, and Henrich CJ. Quassinoid inhibition of AP-1 function does not correlate with cytotoxicity or protein synthesis inhibition. J Nat Prod 72: 503-506, 2009.

16. Blum R and Kloog Y. Metabolism addiction in pancreatic cancer. Cell death \& disease 5: e1065, 2014.

17. Boj SF, Hwang CI, Baker LA, Chio, II, Engle DD, Corbo V, Jager M, Ponz-Sarvise M, Tiriac H, Spector MS, Gracanin A, Oni T, Yu KH, van Boxtel R, Huch M, Rivera KD, Wilson JP, Feigin ME, Ohlund D, Handly-Santana A, Ardito-Abraham CM, Ludwig M, Elyada E, Alagesan B, Biffi G, Yordanov GN, Delcuze B, Creighton B, Wright K, Park Y, Morsink FH, Molenaar IQ, Borel Rinkes IH, Cuppen E, Hao Y, Jin Y, Nijman IJ, Iacobuzio-Donahue C, Leach SD, Pappin DJ, Hammell M, Klimstra DS, Basturk O, Hruban RH, Offerhaus GJ, Vries RG, Clevers H, and Tuveson DA. Organoid models of human and mouse ductal pancreatic cancer. Cell 160: 324-338, 2015.

18. Bokoch GM. Biology of the p21-activated kinases. Annual review of biochemistry 72 : 743-781, 2003.

19. Borad MJ, Reddy SG, Bahary N, Uronis HE, Sigal D, Cohn AL, Schelman WR, Stephenson J, Jr., Chiorean EG, Rosen PJ, Ulrich B, Dragovich T, Del Prete SA, Rarick M, Eng C, Kroll S, and Ryan DP. Randomized Phase II Trial of Gemcitabine Plus TH-302 Versus Gemcitabine in Patients With Advanced Pancreatic Cancer. Journal of clinical oncology : official journal of the American Society of Clinical Oncology 33: 1475-1481, 2015. 20. Bornfeldt KE. Stressing Rac, Ras, and downstream heat shock protein 70. Circulation research 86: 1101-1103, 2000. 


\section{REFERENCES}

21. Brown JL, Stowers L, Baer M, Trejo J, Coughlin S, and Chant J. Human Ste20 homologue hPAK1 links GTPases to the JNK MAP kinase pathway. Current biology : $C B$ 6: 598-605, 1996.

22. Buchwald G, Hostinova E, Rudolph MG, Kraemer A, Sickmann A, Meyer HE, Scheffzek K, and Wittinghofer A. Conformational switch and role of phosphorylation in PAK activation. Molecular and cellular biology 21: 5179-5189, 2001.

23. Caldas $\mathbf{C}$ and Kern SE. K-ras mutation and pancreatic adenocarcinoma. International journal of pancreatology : official journal of the International Association of Pancreatology 18: 1-6, 1995.

24. Chatterjee D, Katz MH, Rashid A, Varadhachary GR, Wolff RA, Wang H, Lee JE, Pisters PW, Vauthey JN, Crane C, Gomez HF, Abbruzzese JL, Fleming JB, and Wang H. Histologic grading of the extent of residual carcinoma following neoadjuvant chemoradiation in pancreatic ductal adenocarcinoma: a predictor for patient outcome. Cancer 118: 3182-3190, 2012.

25. Chauhan SC, Ebeling MC, Maher DM, Koch MD, Watanabe A, Aburatani H, Lio Y, and Jaggi M. MUC13 mucin augments pancreatic tumorigenesis. Molecular cancer therapeutics 11: 24-33, 2012.

26. Chauhan VP, Martin JD, Liu H, Lacorre DA, Jain SR, Kozin SV, Stylianopoulos T, Mousa AS, Han X, Adstamongkonkul P, Popovic Z, Huang P, Bawendi MG, Boucher Y, and Jain RK. Angiotensin inhibition enhances drug delivery and potentiates chemotherapy by decompressing tumour blood vessels. Nature communications 4: 2516, 2013.

27. Chen S, Auletta T, Dovirak O, Hutter C, Kuntz K, El-ftesi S, Kendall J, Han H, Von Hoff DD, Ashfaq R, Maitra A, Iacobuzio-Donahue CA, Hruban RH, and Lucito R. Copy number alterations in pancreatic cancer identify recurrent PAK4 amplification. Cancer biology \& therapy 7: 1793-1802, 2008.

28. Chen S, Yin X, Zhu X, Yan J, Ji S, Chen C, Cai M, Zhang S, Zong H, Hu Y, Yuan Z, Shen Z, and Gu J. The C-terminal kinase domain of the p34cdc2-related PITSLRE protein kinase (p110C) associates with p21-activated kinase 1 and inhibits its activity during anoikis. The Journal of biological chemistry 278: 20029-20036, 2003.

29. Chou TC and Talalay P. Quantitative analysis of dose-effect relationships: the combined effects of multiple drugs or enzyme inhibitors. Advances in enzyme regulation 22: 27-55, 1984.

30. Chow HY, Jubb AM, Koch JN, Jaffer ZM, Stepanova D, Campbell DA, Duron SG, O'Farrell M, Cai KQ, Klein-Szanto AJ, Gutkind JS, Hoeflich KP, and Chernoff J. 


\section{REFERENCES}

p21-Activated kinase 1 is required for efficient tumor formation and progression in a Rasmediated skin cancer model. Cancer research 72: 5966-5975, 2012.

31. Chugh R, Sangwan V, Patil SP, Dudeja V, Dawra RK, Banerjee S, Schumacher RJ, Blazar BR, Georg GI, Vickers SM, and Saluja AK. A preclinical evaluation of Minnelide as a therapeutic agent against pancreatic cancer. Science translational medicine 4: 156ra139, 2012.

32. Ciliberto D, Botta C, Correale P, Rossi M, Caraglia M, Tassone P, and Tagliaferri P. Role of gemcitabine-based combination therapy in the management of advanced pancreatic cancer: a meta-analysis of randomised trials. European journal of cancer 49: 593-603, 2013.

33. Coleman $\mathbf{N}$ and Kissil J. Recent advances in the development of p21-activated kinase inhibitors. Cellular logistics 2: 132-135, 2012.

34. Conroy T, Desseigne F, Ychou M, Bouche O, Guimbaud R, Becouarn Y, Adenis A, Raoul JL, Gourgou-Bourgade S, de la Fouchardiere C, Bennouna J, Bachet JB, Khemissa-Akouz F, Pere-Verge D, Delbaldo C, Assenat E, Chauffert B, Michel P, Montoto-Grillot C, Ducreux M, Groupe Tumeurs Digestives of U, and Intergroup P. FOLFIRINOX versus gemcitabine for metastatic pancreatic cancer. The New England journal of medicine 364: 1817-1825, 2011.

35. Corbett TH, Roberts BJ, Leopold WR, Peckham JC, Wilkoff LJ, Griswold DP, Jr., and Schabel FM, Jr. Induction and chemotherapeutic response of two transplantable ductal adenocarcinomas of the pancreas in C57BL/6 mice. Cancer research 44: 717-726, 1984.

36. De La OJ and Murtaugh LC. Notch and Kras in pancreatic cancer: at the crossroads of mutation, differentiation and signaling. Cell cycle 8: 1860-1864, 2009.

37. de Sousa Cavalcante $\mathbf{L}$ and Monteiro G. Gemcitabine: metabolism and molecular mechanisms of action, sensitivity and chemoresistance in pancreatic cancer. European journal of pharmacology 741: 8-16, 2014.

38. Deacon SW, Beeser A, Fukui JA, Rennefahrt UE, Myers C, Chernoff J, and Peterson JR. An isoform-selective, small-molecule inhibitor targets the autoregulatory mechanism of p21-activated kinase. Chemistry \& biology 15: 322-331, 2008.

39. Deer EL, Gonzalez-Hernandez J, Coursen JD, Shea JE, Ngatia J, Scaife CL, Firpo MA, and Mulvihill SJ. Phenotype and genotype of pancreatic cancer cell lines. Pancreas 39: 425-435, 2010.

40. Deguchi A, Miyoshi H, Kojima Y, Okawa K, Aoki M, and Taketo MM. LKB1 suppresses p21-activated kinase-1 (PAK1) by phosphorylation of Thr109 in the p21-binding domain. The Journal of biological chemistry 285: 18283-18290, 2010. 


\section{REFERENCES}

41. Diyabalanage T, Ratnayake R, Wilson JA, Henrich CJ, Beutler JA, Colburn NH, McMahon JB, and Gustafson KR. Nothospondin, a new AP-1 inhibitory quassinoid from the Cameroonian plant Nothospondias staudtii. 21: 4397-4399, 2011.

42. Dolensek J, Rupnik MS, and Stozer A. Structural similarities and differences between the human and the mouse pancreas. Islets 7: e1024405, 2015.

43. Doppler H, Bastea LI, Borges S, Spratley SJ, Pearce SE, and Storz P. Protein kinase d isoforms differentially modulate cofilin-driven directed cell migration. PloS one 9: e98090, 2014.

44. Dummler B, Ohshiro K, Kumar R, and Field J. Pak protein kinases and their role in cancer. Cancer metastasis reviews 28: 51-63, 2009.

45. Duner S, Lopatko Lindman J, Ansari D, Gundewar C, and Andersson R. Pancreatic cancer: the role of pancreatic stellate cells in tumor progression. Pancreatology: official journal of the International Association of Pancreatology 10: 673-681, 2010.

46. Ebrahimi S, Ghasemi F, Hassanian SM, Shahidsales S, Mardani R, Akbarzade H, Parizadeh SM, Gholamin S, Soleimani A, Ghayour-Mobarhan M, and Avan A. Circulating microRNAs as novel potential diagnostic and prognosis biomarkers in pancreatic cancer. Current pharmaceutical design, 2016.

47. Edwards DC, Sanders LC, Bokoch GM, and Gill GN. Activation of LIM-kinase by Pak1 couples Rac/Cdc42 GTPase signalling to actin cytoskeletal dynamics. Nature cell biology 1: $253-259,1999$.

48. Erez N, Truitt M, Olson P, Arron ST, and Hanahan D. Cancer-Associated Fibroblasts Are Activated in Incipient Neoplasia to Orchestrate Tumor-Promoting Inflammation in an NF-kappaB-Dependent Manner. Cancer cell 17: 135-147, 2010.

49. Erkan M, Adler G, Apte MV, Bachem MG, Buchholz M, Detlefsen S, Esposito I, Friess H, Gress TM, Habisch HJ, Hwang RF, Jaster R, Kleeff J, Kloppel G, Kordes C, Logsdon CD, Masamune A, Michalski CW, Oh J, Phillips PA, Pinzani M, Reiser-Erkan C, Tsukamoto H, and Wilson J. StellaTUM: current consensus and discussion on pancreatic stellate cell research. Gut 61: 172-178, 2012.

50. Erkan M, Hausmann S, Michalski CW, Fingerle AA, Dobritz M, Kleeff J, and Friess H. The role of stroma in pancreatic cancer: diagnostic and therapeutic implications. Nature reviews Gastroenterology \& hepatology 9: 454-467, 2012.

51. Erkan M, Reiser-Erkan C, Michalski CW, Deucker S, Sauliunaite D, Streit S, Esposito I, Friess H, and Kleeff J. Cancer-stellate cell interactions perpetuate the hypoxiafibrosis cycle in pancreatic ductal adenocarcinoma. Neoplasia 11: 497-508, 2009. 


\section{REFERENCES}

52. Erkan M, Reiser-Erkan C, Michalski CW, Kong B, Esposito I, Friess H, and Kleeff J. The impact of the activated stroma on pancreatic ductal adenocarcinoma biology and therapy resistance. Current molecular medicine 12: 288-303, 2012.

53. Eswaran J, Soundararajan M, Kumar R, and Knapp S. UnPAKing the class differences among p21-activated kinases. Trends in biochemical sciences 33: 394-403, 2008.

54. Farrow B, Thomas RP, Wang XF, and Evers BM. Activation of conventional PKC isoforms increases expression of the pro-apoptotic protein Bad and TRAIL receptors. International journal of gastrointestinal cancer 32: 63-72, 2002.

55. Feng L, Geisselbrecht Y, Blanck S, Wilbuer A, Atilla-Gokcumen GE, Filippakopoulos P, Kraling K, Celik MA, Harms K, Maksimoska J, Marmorstein R, Frenking G, Knapp S, Essen LO, and Meggers E. Structurally sophisticated octahedral metal complexes as highly selective protein kinase inhibitors. J Am Chem Soc 133: 5976-5986, 2011.

56. Fiaschetti G, Grotzer MA, Shalaby T, Castelletti D, and Arcaro A. Quassinoids: From traditional drugs to new cancer therapeutics. Current medicinal chemistry 18: 316-328, 2011.

57. Field J and Manser E. The PAKs come of age: Celebrating 18 years of discovery. Cellular logistics 2: 54-58, 2012.

58. Frost JA, Swantek JL, Stippec S, Yin MJ, Gaynor R, and Cobb MH. Stimulation of NFkappa B activity by multiple signaling pathways requires PAK1. The Journal of biological chemistry 275: 19693-19699, 2000.

59. Fujiwara M, Kanayama K, Hirokawa YS, and Shiraishi T. ASF-4-1 fibroblast-rich culture increases chemoresistance and mTOR expression of pancreatic cancer BxPC-3 cells at the invasive front in vitro, and promotes tumor growth and invasion in vivo. Oncology letters 11: 2773-2779, 2016.

60. Galvan JA, Zlobec I, Wartenberg M, Lugli A, Gloor B, Perren A, and Karamitopoulou E. Expression of E-cadherin repressors SNAIL, ZEB1 and ZEB2 by tumour and stromal cells influences tumour-budding phenotype and suggests heterogeneity of stromal cells in pancreatic cancer. British journal of cancer 112: 1944-1950, 2015.

61. Gasman S, Chasserot-Golaz S, Malacombe M, Way M, and Bader MF. Regulated exocytosis in neuroendocrine cells: a role for subplasmalemmal Cdc42/N-WASP-induced actin filaments. Molecular biology of the cell 15: 520-531, 2004. 


\section{REFERENCES}

62. Gerthoffer WT and Gunst SJ. Invited review: focal adhesion and small heat shock proteins in the regulation of actin remodeling and contractility in smooth muscle. Journal of applied physiology 91: 963-972, 2001.

63. Ghosh PC, Larrahondo JE, LeQuesne PW, and Raffauf RF. Antitumor plants. IV. Constituents of Simarouba versicolor. Lloydia 40: 364-369, 1977.

64. Giessrigl B, Krieger S, Rosner M, Huttary N, Saiko P, Alami M, Messaoudi S, Peyrat JF, Maciuk A, Gollinger M, Kopf S, Kazlauskas E, Mazal P, Szekeres T, Hengstschlager M, Matulis D, Jager W, and Krupitza G. Hsp90 stabilizes Cdc25A and counteracts heat shock-mediated Cdc25A degradation and cell-cycle attenuation in pancreatic carcinoma cells. Human molecular genetics 21: 4615-4627, 2012.

65. Giroux V, Iovanna JL, Garcia S, and Dagorn JC. Combined inhibition of PAK7, MAP3K7 and CK2alpha kinases inhibits the growth of MiaPaCa2 pancreatic cancer cell xenografts. Cancer gene therapy 16: 731-740, 2009.

66. Gnesutta N, Qu J, and Minden A. The serine/threonine kinase PAK4 prevents caspase activation and protects cells from apoptosis. The Journal of biological chemistry 276: 1441414419, 2001.

67. Goh KY, Ng NW, Hagen T, and Inoue T. p21-activated kinase interacts with Wnt signaling to regulate tissue polarity and gene expression. Proceedings of the National Academy of Sciences of the United States of America 109: 15853-15858, 2012.

68. Gopinathan A, Morton JP, Jodrell DI, and Sansom OJ. GEMMs as preclinical models for testing pancreatic cancer therapies. Disease models \& mechanisms 8: 1185-1200, 2015 .

69. Grise F, Bidaud A, and Moreau V. Rho GTPases in hepatocellular carcinoma. Biochimica et Biophysica Acta (BBA) - Reviews on Cancer 1795: 137-151, 2009.

70. Guan J and Chen J. [Mutant K-ras gene in pathogenesis of pancreatic ductal adenocarcinoma]. Zhonghua bing li xue za zhi Chinese journal of pathology 41: 62-65, 2012.

71. Guo C, McAlpine I, Zhang J, Knighton DD, Kephart S, Johnson MC, Li H, Bouzida D, Yang A, Dong L, Marakovits J, Tikhe J, Richardson P, Guo LC, Kania R, Edwards MP, Kraynov E, Christensen J, Piraino J, Lee J, Dagostino E, Del-Carmen C, Deng YL, Smeal T, and Murray BW. Discovery of pyrroloaminopyrazoles as novel PAK inhibitors. Journal of medicinal chemistry 55: 4728-4739, 2012.

72. Gurumurthy S and Bardeesy N. Uncapping NF-kappaB activity in pancreatic cancer. The EMBO journal 30: 1-2, 2011. 


\section{REFERENCES}

73. Hamada S, Masamune A, Miura S, Satoh K, and Shimosegawa T. miR-365 induces gemcitabine resistance in pancreatic cancer cells by targeting the adaptor protein SHC1 and pro-apoptotic regulator BAX. Cellular signalling, 2013.

74. Hamada S, Masamune A, and Shimosegawa T. Alteration of pancreatic cancer cell functions by tumor-stromal cell interaction. Frontiers in physiology 4: 318, 2013.

75. Han J, Wang F, Yuan SQ, Guo Y, Zeng ZL, Li LR, Yang J, Wang DS, Liu MY, Zhao H, Liu KY, Liao JW, Zou QF, and Xu RH. Reduced expression of p21-activated protein kinase 1 correlates with poor histological differentiation in pancreatic cancer. $B M C$ cancer 14: 650, 2014.

76. Han S, Delitto D, Zhang D, Sorenson HL, Sarosi GA, Thomas RM, Behrns KE, Wallet SM, Trevino JG, and Hughes SJ. Primary outgrowth cultures are a reliable source of human pancreatic stellate cells. Laboratory investigation; a journal of technical methods and pathology 95: 1331-1340, 2015.

77. Hanlon L, Avila JL, Demarest RM, Troutman S, Allen M, Ratti F, Rustgi AK, Stanger B, Radtke F, Adsay V, Long F, Capobianco AJ, and Kissil JL. Notch1 Functions as a Tumor Suppressor in a Model of K-ras-Induced Pancreatic Ductal Adenocarcinoma. Cancer research 70: 4280-4286, 2010.

78. Haqq J, Howells LM, Garcea G, Metcalfe MS, Steward WP, and Dennison AR. Pancreatic stellate cells and pancreas cancer: current perspectives and future strategies. European journal of cancer 50: 2570-2582, 2014.

79. Hariharan D, Saied A, and Kocher HM. Analysis of mortality rates for pancreatic cancer across the world. $H P B$ : the official journal of the International Hepato Pancreato Biliary Association 10: 58-62, 2008.

80. Hayden MS and Ghosh S. Signaling to NF-kappaB. Genes \& development 18: 21952224, 2004.

81. He H and Baldwin GS. p21-activated kinases and gastrointestinal cancer. Biochimica et biophysica acta 1833: 33-39, 2013.

82. He H, Hirokawa Y, Gazit A, Yamashita Y, Mano H, Kawakami Y, Kawakami, Hsieh CY, Kung HJ, Lessene G, Baell J, Levitzki A, and Maruta H. The Tyr-kinase inhibitor AG879, that blocks the ETK-PAK1 interaction, suppresses the RAS-induced PAK1 activation and malignant transformation. Cancer biology \& therapy 3: 96-101, 2004.

83. He H, Huynh N, Liu KH, Malcontenti-Wilson C, Zhu J, Christophi C, Shulkes A, and Baldwin GS. P-21 activated kinase 1 knockdown inhibits beta-catenin signalling and blocks colorectal cancer growth. 2012 . 


\section{REFERENCES}

84. Hemminki A, Markie D, Tomlinson I, Avizienyte E, Roth S, Loukola A, Bignell G, Warren W, Aminoff M, Hoglund P, Jarvinen H, Kristo P, Pelin K, Ridanpaa M, Salovaara R, Toro T, Bodmer W, Olschwang S, Olsen AS, Stratton MR, de la Chapelle A, and Aaltonen LA. A serine/threonine kinase gene defective in Peutz-Jeghers syndrome. Nature 391: 184-187, 1998.

85. Herreros-Villanueva M, Hijona E, Cosme A, and Bujanda L. Mouse models of pancreatic cancer. World journal of gastroenterology: WJG 18: 1286-1294, 2012.

86. Hingorani SR, Harris WP, Seery TE, Zheng L, Sigal D, Hendifar AE, Braiteh FS, Zalupski M, Baron AD, Bahary N, Wang-Gillam A, LoConte NK, Springett GM, Ritch PS, Hezel AF, Ma WW, Bathini VG, Wu XW, Jiang P, and J. BA. Interim results of a randomized phase II study of PEGPH20 added to nab-paclitaxel/gemcitabine in patients with stage IV previously untreated pancreatic cancer. In: Journal of Clinical Oncology, 2016, p. 439.

87. Hirokawa Y, Arnold M, Nakajima H, Zalcberg J, and Maruta H. Signal therapy of breast cancers by the HDAC inhibitor FK228 that blocks the activation of PAK1 and abrogates the tamoxifen-resistance. Cancer biology \& therapy 4: 956-960, 2005.

88. Hoefen RJ and Berk BC. The multifunctional GIT family of proteins. Journal of cell science 119: 1469-1475, 2006.

89. Hoffmann AC, Mori R, Vallbohmer D, Brabender J, Klein E, Drebber U, Baldus SE, Cooc J, Azuma M, Metzger R, Hoelscher AH, Danenberg KD, Prenzel KL, and Danenberg PV. High expression of HIF1a is a predictor of clinical outcome in patients with pancreatic ductal adenocarcinomas and correlated to PDGFA, VEGF, and bFGF. Neoplasia 10: 674-679, 2008.

90. Hofmann C, Shepelev M, and Chernoff J. The genetics of Pak. Journal of cell science 117: 4343-4354, 2004.

91. Hong SM, Park JY, Hruban RH, and Goggins M. Molecular signatures of pancreatic cancer. Arch Pathol Lab Med 135: 716-727, 2011.

92. Hu X, Guo J, Zheng L, Li C, Zheng TM, Tanyi JL, Liang S, Benedetto C, Mitidie ri M, Katsaros D, Zhao X, Zhang Y, Huang Q, and Zhang L. The heterochronic microRNA let-7 inhibits cell motility by regulating the genes in the actin cytoskeleton pathway in breast cancer. Molecular cancer research : MCR 11:240-250, 2013.

93. Huang C, Wang WM, Gong JP, and Yang K. Oncogenesis and the Clinical Significance of K-ras in Pancreatic Adenocarcinoma. Asian Pacific journal of cancer prevention: APJCP 14: 2699-2701, 2013. 


\section{REFERENCES}

94. Huynh N, Beutler JA, Shulkes A, Baldwin GS, and He H. Glaucarubinone inhibits colorectal cancer growth by suppression of hypoxia-inducible factor 1alpha and beta-catenin via a p-21 activated kinase 1-dependent pathway. Biochimica et biophysica acta 1853: 157$165,2015$.

95. Huynh N, Liu KH, Baldwin GS, and He H. P21-activated kinase 1 stimulates colon cancer cell growth and migration/invasion via ERK- and AKT-dependent pathways. Biochimica et biophysica acta 1803: 1106-1113, 2010.

96. Hwang HW and Mendell JT. MicroRNAs in cell proliferation, cell death, and tumorigenesis. British journal of cancer 96 Suppl: R40-44, 2007.

97. Hwang RF, Moore T, Arumugam T, Ramachandran V, Amos KD, Rivera A, Ji B, Evans DB, and Logsdon CD. Cancer-associated stromal fibroblasts promote pancreatic tumor progression. Cancer research 68: 918-926, 2008.

98. Hwang RF, Moore TT, Hattersley MM, Scarpitti M, Yang B, Devereaux E, Ramachandran V, Arumugam T, Ji B, Logsdon CD, Brown JL, and Godin R. Inhibition of the hedgehog pathway targets the tumor-associated stroma in pancreatic cancer. Molecular cancer research : MCR 10: 1147-1157, 2012.

99. Ide T, Kitajima Y, Miyoshi A, Ohtsuka T, Mitsuno M, Ohtaka K, and Miyazaki

K. The hypoxic environment in tumor-stromal cells accelerates pancreatic cancer progression via the activation of paracrine hepatocyte growth factor/c-Met signaling. Annals of surgical oncology 14: 2600-2607, 2007.

100. Ikebe T, Nakayama H, Shinohara M, and Shirasuna K. NF-kappaB involvement in tumor-stroma interaction of squamous cell carcinoma. Oral oncology 40: 1048-1056, 2004.

101. Jaffer ZM and Chernoff J. p21-activated kinases: three more join the Pak. The international journal of biochemistry \& cell biology 34: 713-717, 2002.

102. Jagadeeshan S, Krishnamoorthy YR, Singhal M, Subramanian A, Mavuluri J, Lakshmi A, Roshini A, Baskar G, Ravi M, Joseph LD, Sadasivan K, Krishnan A, Nair AS, Venkatraman G, and Rayala SK. Transcriptional regulation of fibronectin by p21activated kinase-1 modulates pancreatic tumorigenesis. Oncogene 34: 455-464, 2014.

103. Jagadeeshan S, Subramanian A, Tentu S, Beesetti S, Singhal M, Raghavan S, Surabhi RP, Mavuluri J, Bhoopalan H, Biswal J, Pitani RS, Chidambaram S, Sundaram S, Malathi R, Jeyaraman J, Nair AS, Venkatraman G, and Rayala SK. P21-activated kinase 1 (Pak1) signaling influences therapeutic outcome in pancreatic cancer. Annals of oncology: official journal of the European Society for Medical Oncology / ESMO, 2016. 


\section{REFERENCES}

104. Ji Z, Mei FC, Xie J, and Cheng X. Oncogenic KRAS activates hedgehog signaling pathway in pancreatic cancer cells. The Journal of biological chemistry 282: 14048-14055, 2007.

105. Kakizaki Y, Makino N, Tozawa T, Honda T, Matsuda A, Ikeda Y, Ito M, Saito Y, Kimura W, and Ueno Y. Stromal Fibrosis and Expression of Matricellular Proteins Correlate With Histological Grade of Intraductal Papillary Mucinous Neoplasm of the Pancreas. Pancreas 45: 1145-1152, 2016.

106. Kaneko K, Satoh K, Masamune A, Satoh A, and Shimosegawa T. Myosin light chain kinase inhibitors can block invasion and adhesion of human pancreatic cancer cell lines. Pancreas 24: 34-41, 2002.

107. Karthikeyan S, Hoti SL, Nazeer Y, and Hegde HV. Glaucarubinone sensitizes KB cells to paclitaxel by inhibiting $\mathrm{ABC}$ transporters via ROS-dependent and p53-mediated activation of apoptotic signaling pathways. Oncotarget, 2016.

108. Kelly ML and Chernoff J. Mouse models of PAK function. 2: 84-88, 2012.

109. Kiang JG and Tsokos GC. Heat shock protein $70 \mathrm{kDa}$ : molecular biology, biochemistry, and physiology. Pharmacology \& therapeutics 80: 183-201, 1998.

110. Kikuta K, Masamune A, Watanabe T, Ariga H, Itoh H, Hamada S, Satoh K, Egawa S, Unno M, and Shimosegawa T. Pancreatic stellate cells promote epithelialmesenchymal transition in pancreatic cancer cells. Biochemical and biophysical research communications 403: 380-384, 2010.

111. Kimmelman AC, Hezel AF, Aguirre AJ, Zheng H, Paik JH, Ying H, Chu GC, Zhang JX, Sahin E, Yeo G, Ponugoti A, Nabioullin R, Deroo S, Yang S, Wang X, McGrath JP, Protopopova M, Ivanova E, Zhang J, Feng B, Tsao MS, Redston M, Protopopov A, Xiao Y, Futreal PA, Hahn WC, Klimstra DS, Chin L, and DePinho RA. Genomic alterations link Rho family of GTPases to the highly invasive phenotype of pancreas cancer. Proceedings of the National Academy of Sciences of the United States of America 105: 19372-19377, 2008.

112. Kiosses WB, Hood J, Yang S, Gerritsen ME, Cheresh DA, Alderson N, and Schwartz MA. A dominant-negative p65 PAK peptide inhibits angiogenesis. Circulation research 90: 697-702, 2002.

113. Kissil JL, Wilker EW, Johnson KC, Eckman MS, Yaffe MB, and Jacks T. Merlin, the product of the Nf2 tumor suppressor gene, is an inhibitor of the p21-activated kinase, Pak1. Molecularcell 12: 841-849, 2003. 


\section{REFERENCES}

114. Koh CG, Tan EJ, Manser E, and Lim L. The p21-activated kinase PAK is negatively regulated by POPX1 and POPX2, a pair of serine/threonine phosphatases of the PP2C family. Current biology : CB 12: 317-321, 2002.

115. Kumar R, Gururaj AE, and Barnes CJ. p21-activated kinases in cancer. Nature reviews Cancer 6: 459-471, 2006.

116. Kumar R and Vadlamudi RK. Emerging functions of p21-activated kinases in human cancer cells. Journal of cellular physiology 193: 133-144, 2002.

117. Kuramitsu Y, Wang Y, Taba K, Suenaga S, Ryozawa S, Kaino S, Sakaida I, and Nakamura K. Heat-shock protein 27 plays the key role in gemcitabine-resistance of pancreatic cancer cells. Anticancer research 32: 2295-2299, 2012.

118. Lee SH, Jung YS, Chung JY, Oh AY, Lee SJ, Choi DH, Jang SM, Jang KS, Paik SS, Ha NC, and Park BJ. Novel tumor suppressive function of Smad4 in serum starvationinduced cell death through PAK1-PUMA pathway. Cell death \& disease 2: e235, 2011.

119. Lei J, Huo X, Duan W, Xu Q, Li R, Ma J, Li X, Han L, Li W, Sun H, Wu E, and Ma Q. alpha-Mangostin inhibits hypoxia-driven ROS-induced PSC activation and pancreatic cancer cell invasion. Cancer letters 347: 129-138, 2014.

120. Lei M, Lu W, Meng W, Parrini MC, Eck MJ, Mayer BJ, and Harrison SC. Structure of PAK1 in an autoinhibited conformation reveals a multistage activation switch. Cell 102: 387-397, 2000.

121. Li X and Minden A. PAK4 functions in tumor necrosis factor (TNF) alpha-induced survival pathways by facilitating TRADD binding to the TNF receptor. The Journal of biological chemistry 280: 41192-41200, 2005.

122. Li Y, Shao Y, Tong Y, Shen T, Zhang J, Gu H, and Li F. Nucleo-cytoplasmic shuttling of PAK4 modulates beta-catenin intracellular translocation and signaling. Biochimica et biophysica acta 1823: 465-475, 2012.

123. Licciulli S, Maksimoska J, Zhou C, Troutman S, Kota S, Liu Q, Duron S, Campbell D, Chernoff J, Field J, Marmorstein R, and Kissil JL. FRAX597, a Small Molecule Inhibitor of the p21-activated Kinases, Inhibits Tumorigenesis of Neurofibromatosis Type 2 (NF2)-associated Schwannomas. The Journal of biological chemistry 288: 2910529114, 2013.

124. Lin Y, Richards FM, Krippendorff BF, Bramhall JL, Harrington JA, Bapiro TE, Robertson A, Zheleva D, and Jodrell DI. Paclitaxel and CYC3, an aurora kinase A inhibitor, synergise in pancreatic cancer cells but not bone marrow precursor cells. British journal of cancer 107: 1692-1701, 2012. 


\section{REFERENCES}

125. Linder S, Castanos-Velez E, von Rosen A, and Biberfeld P. Immunohistochemical expression of extracellular matrix proteins and adhesion molecules in pancreatic carcinoma. Hepato-gastroenterology 48: 1321-1327, 2001.

126. Ling J, Kang Y, Zhao R, Xia Q, Lee DF, Chang Z, Li J, Peng B, Fleming JB, Wang H, Liu J, Lemischka IR, Hung MC, and Chiao PJ. KrasG12D-induced IKK2/beta/NFkappaB activation by IL-1alpha and p62 feedforward loops is required for development of pancreatic ductal adenocarcinoma. Cancer cell 21: 105-120, 2012.

127. Ling Q, Xu X, Zheng S-S, and Kalthoff $\mathbf{H}$. The diversity between pancreatic head and body/tail cancers: clinical parameters and in vitro models. Hepatobiliary \& Pancreatic Diseases International: HBPD INT 12: 480-487, 2013.

128. Liu KH, Huynh N, Patel O, Shulkes A, Baldwin G, and He H. P21-activated kinase 1 promotes colorectal cancer survival by up-regulation of hypoxia-inducible factor-1alpha. Cancer letters 340: 22-29, 2013.

129. Long J, Zhang Y, Yu X, Yang J, LeBrun DG, Chen C, Yao Q, and Li M. Overcoming drug resistance in pancreatic cancer. Expert opinion on therapeutic targets 15 : 817-828, 2011.

130. Lu J, Getz G, Miska EA, Alvarez-Saavedra E, Lamb J, Peck D, Sweet-Cordero A, Ebert BL, Mak RH, Ferrando AA, Downing JR, Jacks T, Horvitz HR, and Golub TR. MicroRNA expression profiles classify human cancers. Nature 435: 834-838, 2005.

131. Ma J, Xia J, Miele L, Sarkar FH, and Wang Z. Notch Signaling Pathway in Pancreatic Cancer Progression. Pancreatic disorders \& therapy 3, 2013.

132. Maehama T and Dixon JE. The tumor suppressor, PTEN/MMAC1, dephosphorylates the lipid second messenger, phosphatidylinositol 3,4,5-trisphosphate. The Journal of biological chemistry 273: 13375-13378, 1998.

133. Mahlamaki EH, Kauranie mi P, Monni O, Wolf M, Hautaniemi S, and Kallioniemi A. High-resolution genomic and expression profiling reveals 105 putative amplification target genes in pancreatic cancer. Neoplasia 6: 432-439, 2004.

134. Maitra A, Fukushima N, Takaori K, and Hruban RH. Precursors to invasive pancreatic cancer. Advances in anatomic pathology 12: 81-91, 2005.

135. Maitra A and Hruban RH. Pancreatic cancer. Annual review of pathology 3: 157-188, 2008.

136. Maitra A, Iacobuzio-Donahue C, Rahman A, Sohn TA, Argani P, Meyer R, Yeo CJ, Cameron JL, Goggins M, Kern SE, Ashfaq R, Hruban RH, and Wilentz RE. Immunohistochemical validation of a novel epithelial and a novel stromal marker of pancreatic 


\section{REFERENCES}

ductal adenocarcinoma identified by global expression microarrays: sea urchin fascin homolog and heat shock protein 47. American journal of clinical pathology 118: 52-59, 2002.

137. Maitra A, Kern SE, and Hruban RH. Molecular pathogenesis of pancreatic cancer. Best Practice \& Research in Clinical Gastroenterology 20: 211-226, 2006.

138. Maksimoska J, Feng L, Harms K, Yi C, Kissil J, Marmorstein R, and Meggers E. Targeting large kinase active site with rigid, bulky octahedral ruthenium complexes. $J$ Am Chem Soc 130: 15764-15765, 2008.

139. Manser E, Leung T, Salihuddin H, Zhao ZS, and Lim L. A brain serine/threonine protein kinase activated by Cdc42 and Rac1. Nature 367: 40-46, 1994.

140. Maroto B, Ye MB, von Lohneysen K, Schnelzer A, and Knaus UG. P21-activated kinase is required for mitotic progression and regulates Plk1. Oncogene 27: 4900-4908, 2008. 141. Maruta H. Herbal therapeutics that block the oncogenic kinase PAK1: a practical approach towards PAK1-dependent diseases and longevity. Phytotherapy research : PTR 28: 656-672, 2014.

142. Maruta H. PAKs, RAC/CDC42 (p21)-activated kinases: towards the cure of cancer and other PAK-dependent diseases / edited by Hiroshi Maruta, NF/TSC Cure Org, Melbourne, Australia: London : Elsevier, 2013.

First edition., 2013.

143. Masamune A, Kikuta K, Watanabe T, Satoh K, Hirota M, and Shimosegawa T. Hypoxia stimulates pancreatic stellate cells to induce fibrosis and angiogenesis in pancreatic cancer. American journal of physiology Gastrointestinal and liver physiology 295: G709-717, 2008 .

144. Masamune A and Shimosegawa T. Signal transduction in pancreatic stellate cells. Journal of gastroenterology 44: 249-260, 2009.

145. McCarroll JA, Naim S, Sharbeen G, Russia N, Lee J, Kavallaris M, Goldstein D, and Phillips PA. Role of pancreatic stellate cells in chemoresistance in pancreatic cancer. Frontiers in physiology 5: 141, 2014.

146. Mehenni H, Resta N, Park JG, Miyaki M, Guanti G, and Costanza MC. Cancer risks in LKB1 germline mutation carriers. Gut 55: 984-990, 2006.

147. Menard RE, Jovanovski AP, and Mattingly RR. Active p21-activated kinase 1 rescues MCF10A breast epithelial cells from undergoing anoikis. Neoplasia 7: 638-645, 2005. 


\section{REFERENCES}

148. Menard RE and Mattingly RR. Cell surface receptors activate p21-activated kinase 1 via multiple Ras and PI3-kinase-dependent pathways. Cellular signalling 15: 1099-1109, 2003.

149. Menges CW, Sementino E, Talarchek J, Xu J, Chernoff J, Peterson JR, and Testa JR. Group I p21-activated kinases (PAKs) promote tumor cell proliferation and survival through the AKT1 and Raf-MAPK pathways. Molecular cancer research : MCR 10: 1178$1188,2012$.

150. Misra UK, Deedwania R, and Pizzo SV. Binding of activated alpha2-macroglobulin to its cell surface receptor GRP78 in 1-LN prostate cancer cells regulates PAK-2-dependent activation of LIMK. The Journal of biological chemistry 280: 26278-26286, 2005.

151. Miyamoto H, Murakami T, Tsuchida K, Sugino H, Miyake H, and Tashiro S. Tumor-stroma interaction of human pancreatic cancer: acquired resistance to anticancer drugs and proliferation regulation is dependent on extracellular matrix proteins. Pancreas 28: 38-44, 2004.

152. Mizuarai S, Kawagishi A, and Kotani H. Inhibition of p70S6K2 down-regulates Hedgehog/GLI pathway in non-small cell lung cancer cell lines. Molecular cancer 8: 44, 2009. 153. Moffitt RA, Marayati R, Flate EL, Volmar KE, Loeza SG, Hoadley KA, Rashid NU, Williams LA, Eaton SC, Chung AH, Smyla JK, Anderson JM, Kim HJ, Bentrem DJ, Talamonti MS, Iacobuzio-Donahue CA, Hollingsworth MA, and Yeh JJ. Virtual microdissection identifies distinct tumor- and stroma-specific subtypes of pancreatic ductal adenocarcinoma. Nature genetics 47: 1168-1178, 2015.

154. Moir JA, Mann J, and White SA. The role of pancreatic stellate cells in pancreatic cancer. Surgical oncology 24: 232-238, 2015.

155. Molli PR, Li DQ, Bagheri-Yarmand R, Pakala SB, Katayama H, Sen S, Iyer J, Chernoff J, Tsai MY, Nair SS, and Kumar R. Arpc1b, a centrosomal protein, is both an activator and substrate of Aurora A. The Journal of cell biology 190: 101-114, 2010.

156. Molli PR, Li DQ, Murray BW, Rayala SK, and Kumar R. PAK signaling in oncogenesis. Oncogene 28: 2545-2555, 2009.

157. Monjour L, Rouquier F, Alfred C, and Polonsky J. [Therapeutic trials of experimental murine malaria with the quassinoid, glaucarubinone]. Comptes rendus de l'Academie des sciences Serie III, Sciences de la vie 304: 129-132, 1987.

158. Moon SU, Kim JW, Sung JH, Kang MH, Kim SH, Chang H, Lee JO, Kim YJ, Lee KW, Kim JH, Bang SM, and Lee JS. p21-Activated Kinase 4 (PAK4) as a Predictive Marker 


\section{REFERENCES}

of Gemcitabine Sensitivity in Pancreatic Cancer Cell Lines. Cancer research and treatment : official journal of Korean Cancer Association, 2014.

159. Morris JPt, Wang SC, and Hebrok M. KRAS, Hedgehog, Wnt and the twisted developmental biology of pancreatic ductal adenocarcinoma. Nature reviews Cancer 10: 683$695,2010$.

160. Mortazavi F, Lu J, Phan R, Lewis M, Trinidad K, Aljilani A, Pezeshkpour G, and Tamanoi F. Significance of KRAS/PAK1/Crk pathway in non-small cell lung cancer oncogenesis. BMC cancer 15: 381, 2015.

161. Morton JP, Jamieson NB, Karim SA, Athineos D, Ridgway RA, Nixon C, McKay CJ, Carter R, Brunton VG, Frame MC, Ashworth A, Oien KA, Evans TR, and Sansom OJ. LKB1 haploinsufficiency cooperates with Kras to promote pancreatic cancer through suppression of p21-dependent growth arrest. Gastroenterology 139: 586-597, 597 e581-586, 2010 .

162. Moser C, Lang SA, Hackl C, Wagner C, Scheiffert E, Schlitt HJ, Geissler EK, and Stoeltzing O. Targeting HSP90 by the novel inhibitor NVP-AUY922 reduces growth and angiogenesis of pancreatic cancer. Anticancer research 32: 2551-2561, 2012.

163. Nagao Y, Hisaoka M, Matsuyama A, Kanemitsu S, Hamada T, Fukuyama T, Nakano R, Uchiyama A, Kawamoto M, Yamaguchi K, and Hashimoto H. Association of microRNA-21 expression with its targets, PDCD4 and TIMP3, in pancreatic ductal adenocarcinoma. Modern pathology : an official journal of the United States and Canadian Academy of Pathology, Inc 25: 112-121, 2012.

164. Neesse A, Algul H, Tuveson DA, and Gress TM. Stromal biology and therapy in pancreatic cancer: a changing paradigm. Gut 64: 1476-1484, 2015.

165. Nheu TV, He H, Hirokawa Y, Tamaki K, Florin L, Schmitz ML, SuzukiTakahashi I, Jorissen RN, Burgess AW, Nishimura S, Wood J, and Maruta H. The K252a derivatives, inhibitors for the PAK/MLK kinase family selectively block the growth of RAS transformants. Cancer journal 8: 328-336, 2002.

166. Nikfarjam M, Yeo D, He H, Baldwin G, Fifis T, Costa P, Tan B, Yang E, Wen SW, and Christophi C. Comparison of Two Syngeneic Orthotopic Murine Models of Pancreatic Adenocarcinoma. Journal of investigative surgery : the official journal of the Academy of Surgical Research 26: 352-359, 2013.

167. Nuche-Berenguer B, Ramos-Alvarez I, and Jensen RT. The p21-activated kinase, PAK2, is important in the activation of numerous pancreatic acinar cell signaling cascades and in the onset of early pancreatitis events. Biochimica et biophysica acta 1862: 1122-1136, 2016. 


\section{REFERENCES}

168. Nwaeburu CC, Bauer N, Zhao Z, Abukiwan A, Gladkich J, Benner A, and Herr I. Up-regulation of microRNA Let-7c by quercetin inhibits pancreatic cancer progression by activation of Numbl. Oncotarget, 2016.

169. Olive KP, Jacobetz MA, Davidson CJ, Gopinathan A, McIntyre D, Honess D, Madhu B, Goldgraben MA, Caldwell ME, Allard D, Frese KK, Denicola G, Feig C, Combs C, Winter SP, Ireland-Zecchini H, Reichelt S, Howat WJ, Chang A, Dhara M, Wang L, Ruckert F, Grutzmann R, Pilarsky C, Izeradjene K, Hingorani SR, Huang P, Davies SE, Plunkett W, Egorin M, Hruban RH, Whitebread N, McGovern K, Adams J, Iacobuzio-Donahue C, Griffiths J, and Tuveson DA. Inhibition of Hedgehog signaling enhances delivery of chemotherapy in a mouse model of pancreatic cancer. Science 324: 1457$1461,2009$.

170. Omary MB, Lugea A, Lowe AW, and Pandol SJ. The pancreatic stellate cell: a star on the rise in pancreatic diseases. The Journal of clinical investigation 117: 50-59, 2007.

171. Ozdemir BC, Pentcheva-Hoang T, Carstens JL, Zheng X, Wu CC, Simpson TR, Laklai H, Sugimoto H, Kahlert C, Novitskiy SV, De Jesus-Acosta A, Sharma P, Heidari P, Mahmood U, Chin L, Moses HL, Weaver VM, Maitra A, Allison JP, LeBleu VS, and Kalluri R. Depletion of carcinoma-associated fibroblasts and fibrosis induces immunosuppression and accelerates pancreas cancer with reduced survival. Cancer cell 25: 719-734, 2014.

172. Pandolfi A, Stanley RF, Yu Y, Bartholdy B, Pendurti G, Gritsman K, Boultwood J, Chernoff J, Verma A, and Steidl U. PAK1 is a therapeutic target in acute myeloid leukemia and myelodysplastic syndrome. Blood 126: 1118-1127, 2015.

173. Pharmaceuticals I. Infinity reports update from Phase 2 study of saridegib plus gemcitabine in patients with metastatic pancreatic cancer.

174. Phillips P. Pancreatic stellate cells and fibrosis. In: Pancreatic Cancer and Tumor Microenvironment, edited by Grippo PJ and Munshi HG. Trivandrum (India), 2012.

175. Porchia LM, Guerra M, Wang YC, Zhang Y, Espinosa AV, Shinohara M, Kulp SK, Kirschner LS, Saji M, Chen CS, and Ringel MD. 2-amino-N-\{4-[5-(2-phenanthrenyl)3-(trifluoromethyl)-1H-pyrazol-1-yl]-phenyl \} acetamide (OSU-03012), a celecoxib derivative, directly targets p21-activated kinase. Molecular pharmacology 72: 1124-1131, 2007.

176. Pothula SP, Xu Z, Goldstein D, Pirola RC, Wilson JS, and Apte MV. Key role of pancreatic stellate cells in pancreatic cancer. Cancer letters, 2015. 


\section{REFERENCES}

177. Qu J, Li X, Novitch BG, Zheng Y, Kohn M, Xie JM, Kozinn S, Bronson R, Beg AA, and Minden A. PAK4 kinase is essential for embryonic viability and for proper neuronal development. Molecular and cellular biology 23: 7122-7133, 2003.

178. Quan M, Cui J, Xia T, Jia Z, Xie D, Wei D, Huang S, Huang Q, Zheng S, and Xie

K. Merlin/NF2 Suppresses Pancreatic Tumor Growth and Metastasis by Attenuating the FOXM1-Mediated Wnt/beta-Catenin Signaling. Cancer research 75: 4778-4789, 2015.

179. Radulovich N, Qian JY, and Tsao MS. Human pancreatic duct epithelial cell model for KRAS transformation. Methods in enzymology 439: 1-13, 2008.

180. Rahib L, Smith BD, Aizenberg R, Rosenzweig AB, Fleshman JM, and Matrisian LM. Projecting cancer incidence and deaths to 2030: the unexpected burden of thyroid, liver, and pancreas cancers in the United States. Cancer research 74: 2913-2921, 2014.

181. Rauhala HE, Teppo S, Niemela S, and Kallioniemi A. Silencing of the ARP2/3 complex disturbs pancreatic cancer cell migration. Anticancer research 33: 45-52, 2013.

182. Rebours V, Albuquerque M, Sauvanet A, Ruszniewski P, Levy P, Paradis V, Bedossa P, and Couvelard A. Hypoxia pathways and cellular stress activate pancreatic stellate cells: development of an organotypic culture model of thick slices of normal human pancreas. PloS one 8: e76229, 2013.

183. Rennefahrt UE, Deacon SW, Parker SA, Devarajan K, Beeser A, Chernoff J, Knapp S, Turk BE, and Peterson JR. Specificity profiling of Pak kinases allows identification of novel phosphorylation sites. The Journal of biological chemistry 282: 1566715678, 2007.

184. Rettig M, Trinidad K, Pezeshkpour G, Frost P, Sharma S, Moatamed F, Tamanoi F, and Mortazavi F. PAK1 kinase promotes cell motility and invasiveness through CRK-II serine phosphorylation in non-small cell lung cancer cells. PloS one 7: e42012, 2012.

185. Reynolds RB and Folloder J. Clinical Management of Pancreatic Cancer. Journal of the advanced practitioner in oncology 5: 356-364, 2014.

186. Rhim AD, Oberstein PE, Thomas DH, Mirek ET, Palermo CF, Sastra SA, Dekleva EN, Saunders T, Becerra CP, Tattersall IW, Westphalen CB, Kitajewski J, Fernandez Barrena MG, Fernandez-Zapico ME, Iacobuzio-Donahue C, Olive KP, and Stanger BZ. Stromal elements act to restrain, rather than support, pancreatic ductal adenocarcinoma. Cancer cell 25: 735-747, 2014.

187. Rhim AD and Stanger BZ. Molecular biology of pancreatic ductal adenocarcinoma progression: aberrant activation of developmental pathways. Progress in molecular biology and translational science 97: 41-78, 2010. 


\section{REFERENCES}

188. Sanchez-Solana B, Motwani M, Li DQ, Eswaran J, and Kumar R. p21-activated kinase-1 signaling regulates transcription of tissue factor and tissue factor pathway inhibitor. The Journal of biological chemistry 287: 39291-39302, 2012.

189. Sanders LC, Matsumura F, Bokoch GM, and de Lanerolle P. Inhibition of myosin light chain kinase by p21-activated kinase. Science 283: 2083-2085, 1999.

190. Sangwan V, Banerjee S, Jensen KM, Chen Z, Chugh R, Dudeja V, Vickers SM, and Saluja AK. Primary and liver metastasis-derived cell lines from KrasG12D; Trp53R172H; Pdx-1 Cre animals undergo apoptosis in response to triptolide. Pancreas 44: 583-589, 2015.

191. Sarkar S, Dubaybo H, Ali S, Goncalves P, Kollepara SL, Sethi S, Philip PA, and Li Y. Down-regulation of miR-221 inhibits proliferation of pancreatic cancer cells through upregulation of PTEN, p27(kip1), p57(kip2), and PUMA. American journal of cancer research 3: 465-477, 2013.

192. Schmitz AAP, Govek E-E, Böttner B, and Van Aelst L. Rho GTPases: Signaling, Migration, and Invasion. Experimental Cell Research 261: 1-12, 2000.

193. Schurmann A, Mooney AF, Sanders LC, Sells MA, Wang HG, Reed JC, and Bokoch GM. p21-activated kinase 1 phosphorylates the death agonist bad and protects cells from apoptosis. Molecular and cellular biology 20: 453-461, 2000.

194. Schutte M, Hruban RH, Geradts J, Maynard R, Hilgers W, Rabindran SK, Moskaluk CA, Hahn SA, Schwarte-Waldhoff I, Schmiegel W, Baylin SB, Kern SE, and Herman JG. Abrogation of the Rb/p16 tumor-suppressive pathway in virtually all pancreatic carcinomas. Cancer research 57: 3126-3130, 1997.

195. Sherman MH, Yu RT, Engle DD, Ding N, Atkins AR, Tiriac H, Collisson EA, Connor F, Van Dyke T, Kozlov S, Martin P, Tseng TW, Dawson DW, Donahue TR, Masamune A, Shimosegawa T, Apte MV, Wilson JS, Ng B, Lau SL, Gunton JE, Wahl GM, Hunter T, Drebin JA, O'Dwyer PJ, Liddle C, Tuveson DA, Downes M, and Evans RM. Vitamin D receptor-mediated stromal reprogramming suppresses pancreatitis and enhances pancreatic cancer therapy. Cell 159: 80-93, 2014.

196. Shi X, Liu S, Kleeff J, Friess H, and Buchler MW. Acquired resistance of pancreatic cancer cells towards 5-Fluorouracil and gemcitabine is associated with altered expression of apoptosis-regulating genes. Oncology 62: 354-362, 2002.

197. Sicard A, Semblat JP, Doerig C, Hamelin R, Moniatte M, Dorin-Semblat D, Spicer JA, Srivastava A, Retzlaff S, Heussler V, Waters AP, and Doerig C. Activation of a PAKMEK signalling pathway in malaria parasite-infected erythrocytes. Cellular microbiology 13: 836-845, 2011. 


\section{REFERENCES}

198. Siegel RL, Miller KD, and Jemal A. Cancer statistics, 2016. CA: a cancer journal for clinicians 66: 7-30, 2016.

199. Singh P, Wig JD, and Srinivasan R. The Smad family and its role in pancreatic cancer. Indian Journal Of Cancer 48: 351-360, 2011.

200. Song B, Liu XS, Rice SJ, Kuang S, Elzey BD, Konieczny SF, Ratliff TL, Hazbun T, Chiorean EG, and Liu X. Plk1 phosphorylation of orc2 and hbo1 contributes to gemcitabine resistance in pancreatic cancer. Molecular cancer therapeutics 12: 58-68, 2013.

201. Sun D, Yu F, Ma Y, Zhao R, Chen X, Zhu J, Zhang CY, Chen J, and Zhang J. MicroRNA-31 activates the RAS pathway and functions as an oncogenic MicroRNA in human colorectal cancer by repressing RAS p21 GTPase activating protein 1 (RASA1). The Journal of biological chemistry 288: 9508-9518, 2013.

202. Takikawa T, Masamune A, Hamada S, Nakano E, Yoshida N, and Shimosegawa T. miR-210 regulates the interaction between pancreatic cancer cells and stellate cells. Biochemical and biophysical research communications 437: 433-439, 2013.

203. Talukder AH, Meng Q, and Kumar R. CRIPak, a novel endogenous Pak1 inhibitor. Oncogene 25: 1311-1319, 2006.

204. Tang Y, Chen Z, Ambrose D, Liu J, Gibbs JB, Chernoff J, and Field J. Kinasedeficient Pak1 mutants inhibit Ras transformation of Rat-1 fibroblasts. Molecular and cellular biology 17: 4454-4464, 1997.

205. Tang Y, Marwaha S, Rutkowski JL, Tennekoon GI, Phillips PC, and Field J. A role for Pak protein kinases in Schwann cell transformation. Proceedings of the National Academy of Sciences of the United States of America 95: 5139-5144, 1998.

206. Teramoto H, Crespo P, Coso OA, Igishi T, Xu N, and Gutkind JS. The small GTPbinding protein rho activates c-Jun $\mathrm{N}$-terminal kinases/stress-activated protein kinases in human kidney 293T cells. Evidence for a Pak-independent signaling pathway. The Journal of biological chemistry 271: 25731-25734, 1996.

207. Torphy RJ, Volmar KE, Naim R, Cader SR, Johansen JS, Hollingsworth MA, Collison EA, and Yeh JJ. Quantification of tumor stroma as a biomarker in pancreatic adenocarcinoma. Journal of clinical oncology : official journal of the American Society of Clinical Oncology 33, 2015.

208. Torres MP, Rachagani S, Souchek JJ, Mallya K, Johansson SL, and Batra SK. Novel pancreatic cancer cell lines derived from genetically engineered mouse models of spontaneous pancreatic adenocarcinoma: applications in diagnosis and therapy. PloS one 8: e80580, 2013. 


\section{REFERENCES}

209. Tseng WW, Winer D, Kenkel JA, Choi O, Shain AH, Pollack JR, French R, Lowy AM, and Engleman EG. Development of an orthotopic model of invasive pancreatic cancer in an immunocompetent murine host. Clinical cancer research : an official journal of the American Association for Cancer Research 16: 3684-3695, 2010.

210. Tyagi N, Bhardwaj A, Singh AP, McClellan S, Carter JE, and Singh S. p-21 activated kinase 4 promotes proliferation and survival of pancreatic cancer cells through AKTand ERK-dependent activation of NF-kappaB pathway. Oncotarget 5: 8778-8789, 2014.

211. Tyagi N, Marimuthu S, Bhardwaj A, Deshmukh SK, Srivastava SK, Singh AP, McClellan S, Carter JE, and Singh S. p-21 activated kinase 4 (PAK4) maintains stem celllike phenotypes in pancreatic cancer cells through activation of STAT3 signaling. Cancer letters 370: 260-267, 2016.

212. Usami Y, Nakagawa-Goto K, Lang JY, Kim Y, Lai CY, Goto M, Sakurai N, Taniguchi M, Akiyama T, Morris-Natschke SL, Bastow KF, Cragg G, Newman DJ, Fujitake M, Takeya K, Hung MC, Lee EY, and Lee KH. Antitumor Agents. 282. 2'-(R)-Oacetylglaucarubinone, a quassinoid from Odyendyea gabonensis as a potential anti-breast and anti-ovarian cancer agent. Journal of natural products 73: 1553-1558, 2010.

213. Vadlamudi RK, Adam L, Wang RA, Mandal M, Nguyen D, Sahin A, Chernoff J, Hung MC, and Kumar R. Regulatable expression of p21-activated kinase-1 promotes anchorage-independent growth and abnormal organization of mitotic spindles in human epithelial breast cancer cells. The Journal of biological chemistry 275: 36238-36244, 2000.

214. Vadlamudi RK, Bagheri-Yarmand R, Yang Z, Balasenthil S, Nguyen D, Sahin AA, den Hollander P, and Kumar R. Dynein light chain 1, a p21-activated kinase 1-interacting substrate, promotes cancerous phenotypes. Cancer cell 5: 575-585, 2004.

215. Vadlamudi RK and Kumar R. P21-activated kinases in human cancer. Cancer metastasis reviews 22: 385-393, 2003.

216. Vadlamudi RK, Li F, Barnes CJ, Bagheri-Yarmand R, and Kumar R. p41-Arc subunit of human Arp2/3 complex is a p21-activated kinase-1-interacting substrate. EMBO reports 5: 154-160, 2004.

217. Valeriote FA, Corbett TH, Grieco PA, Moher ED, Collins JL, and Fleck TJ. Anticancer activity of glaucarubinone analogues. Oncology research 10: 201-208, 1998.

218. Van den Broeke C, Radu M, Chernoff J, and Favoreel HW. An emerging role for p21-activated kinases (Paks) in viral infections. Trends in cell biology 20: 160-169, 2010. 


\section{REFERENCES}

219. Verma G, Bhatia H, and Datta M. Gene expression profiling and pathway analysis identify the integrin signaling pathway to be altered by IL-1beta in human pancreatic cancer cells: role of JNK. Cancer letters 320: 86-95, 2012.

220. Viaud J and Peterson JR. An allosteric kinase inhibitor binds the p21-activated kinase autoregulatory domain covalently. Molecular cancer therapeutics 8: 2559-2565, 2009.

221. Vlahopoulos S and Zoumpourlis VC. JNK: a key modulator of intracellular signaling. Biochemistry Biokhimiia 69: 844-854, 2004.

222. Vlecken DH and Bagowski CP. LIMK1 and LIMK2 are important for metastatic behavior and tumor cell-induced angiogenesis of pancreatic cancer cells. Zebrafish 6: 433-439, 2009.

223. Von Hoff DD, Ervin T, Arena FP, Chiorean EG, Infante J, Moore M, Seay T, Tjulandin SA, Ma WW, Saleh MN, Harris M, Reni M, Dowden S, Laheru D, Bahary N, Ramanathan RK, Tabernero J, Hidalgo M, Goldstein D, Van Cutsem E, Wei X, Iglesias J, and Renschler MF. Increased survival in pancreatic cancer with nab-paclitaxel plus gemcitabine. The New England journal of medicine 369: 1691-1703, 2013.

224. Vonlaufen A, Joshi S, Qu C, Phillips PA, Xu Z, Parker NR, Toi CS, Pirola RC, Wilson JS, Goldstein D, and Apte MV. Pancreatic stellate cells: partners in crime with pancreatic cancer cells. Cancer research 68: 2085-2093, 2008.

225. Vonlaufen A, Phillips PA, Xu Z, Goldstein D, Pirola RC, Wilson JS, and Apte MV. Pancreatic stellate cells and pancreatic cancer cells: an unholy alliance. Cancer research 68: 7707-7710, 2008.

226. Waddell N, Pajic M, Patch AM, Chang DK, Kassahn KS, Bailey P, Johns AL, Miller D, Nones K, Quek K, Quinn MC, Robertson AJ, Fadlullah MZ, Bruxner TJ, Christ AN, Harliwong I, Idrisoglu S, Manning S, Nourse C, Nourbakhsh E, Wani S, Wilson PJ, Markham E, Cloonan N, Anderson MJ, Fink JL, Holmes O, Kazakoff SH, Leonard C, Newell F, Poudel B, Song S, Taylor D, Waddell N, Wood S, Xu Q, Wu J, Pinese M, Cowley MJ, Lee HC, Jones MD, Nagrial AM, Humphris J, Chantrill LA, Chin V, Steinmann AM, Mawson A, Humphrey ES, Colvin EK, Chou A, Scarlett CJ, Pinho AV, Giry-Laterriere M, Rooman I, Samra JS, Kench JG, Pettitt JA, Merrett ND, Toon C, Epari K, Nguyen NQ, Barbour A, Zeps N, Jamieson NB, Graham JS, Niclou SP, Bjerkvig R, Grutzmann R, Aust D, Hruban RH, Maitra A, Iacobuzio-Donahue CA, Wolfgang CL, Morgan RA, Lawlor RT, Corbo V, Bassi C, Falconi M, Zamboni G, Tortora G, Tempero MA, Australian Pancreatic Cancer Genome I, Gill AJ, Eshleman JR, Pilarsky C, Scarpa A, 


\section{REFERENCES}

Musgrove EA, Pearson JV, Biankin AV, and Grimmond SM. Whole genomes redefine the mutational landscape of pancreatic cancer. Nature 518: 495-501, 2015.

227. Wang Y, Shi J, Chai K, Ying X, and Zhou BP. The Role of Snail in EMT and Tumorigenesis. Current cancer drug targets 13: 963-972, 2013.

228. Wang Y, Zhang Y, Yang J, Ni X, Liu S, Li Z, Hodges SE, Fisher WE, Brunicardi FC, Gibbs RA, Gingras MC, and Li M. Genomic sequencing of key genes in mouse pancreatic cancer cells. Current molecular medicine 12: 331-341, 2012.

229. Wang Z, Oh E, Clapp DW, Chernoff J, and Thurmond DC. Inhibition or ablation of p21-activated kinase (PAK1) disrupts glucose homeostatic mechanisms in vivo. The Journal of biological chemistry 286: 41359-41367, 2011.

230. Watt $\mathbf{J}$ and Kocher HM. The desmoplastic stroma of pancreatic cancer is a barrier to immune cell infiltration. Oncoimmunology 2: e26788, 2013.

231. Wei W, Li H, Li N, Sun H, Li Q, and Shen X. WNT5A/JNK signaling regulates pancreatic cancer cells migration by Phosphorylating Paxillin. Pancreatology : official journal of the International Association of Pancreatology 13: 384-392, 2013.

232. Weisz Hubsman M, Volinsky N, Manser E, Yablonski D, and Aronheim A. Autophosphorylation-dependent degradation of Pak1, triggered by the Rho-family GTPase, Chp. The Biochemical journal 404: 487-497, 2007.

233. Westwick JK, Lambert QT, Clark GJ, Symons M, Van Aelst L, Pestell RG, and Der CJ. Rac regulation of transformation, gene expression, and actin organization by multiple, PAK-independent pathways. Molecular and cellular biology 17: 1324-1335, 1997.

234. Whitcomb DC. Inflammation and Cancer V. Chronic pancreatitis and pancreatic cancer. American journal of physiology Gastrointestinal and liver physiology 287: G315-319, 2004.

235. Wu WS. The signaling mechanism of ROS in tumor progression. Cancer metastasis reviews 25: 695-705, 2006.

236. Xia C, Ma W, Stafford LJ, Marcus S, Xiong WC, and Liu M. Regulation of the p21activated kinase (PAK) by a human Gbeta -like WD-repeat protein, hPIP1. Proceedings of the National Academy of Sciences of the United States of America 98: 6174-6179, 2001.

237. Xu J, Liu H, Chen L, Wang S, Zhou L, Yun X, Sun L, Wen Y, and Gu J. Hepatitis $\mathrm{B}$ virus $\mathrm{X}$ protein confers resistance of hepatoma cells to anoikis by up-regulating and activating p21-activated kinase 1. Gastroenterology 143: 199-212 e194, 2012. 


\section{REFERENCES}

238. Xu Z, Vonlaufen A, Phillips PA, Fiala-Beer E, Zhang X, Yang L, Biankin AV, Goldstein D, Pirola RC, Wilson JS, and Apte MV. Role of pancreatic stellate cells in pancreatic cancer metastasis. The American journal of pathology 177: 2585-2596, 2010.

239. Xue N, Jin J, Liu D, Yan R, Zhang S, Yu X, and Chen X. Antiproliferative effect of HSP90 inhibitor Y306zh against pancreatic cancer is mediated by interruption of AKT and MAPK signaling pathways. Current cancer drug targets 14: 671-683, 2014.

240. Yamada N, Noguchi S, Mori T, Naoe T, Maruo K, and Akao Y. Tumor-suppressive microRNA-145 targets catenin delta-1 to regulate Wnt/beta-catenin signaling in human colon cancer cells. Cancer letters 335: 332-342, 2013.

241. Yanase S, Luo Y, and Maruta H. PAK1-deficiency/down-regulation reduces brood size, activates HSP16.2 gene and extends lifespan in Caenorhabditis elegans. Drug discoveries \& therapeutics 7: 29-35, 2013.

242. Yang Z, Rayala S, Nguyen D, Vadlamudi RK, Chen S, and Kumar R. Pak1 phosphorylation of snail, a master regulator of epithelial-to-mesenchyme transition, modulates snail's subcellular localization and functions. Cancer research 65: 3179-3184, 2005.

243. Ye DZ and Field J. PAK signaling in cancer. Cellular logistics 2: 105-116, 2012.

244. Yi C, Wilker EW, Yaffe MB, Stemmer-Rachamimov A, and Kissil JL. Validation of the p21-activated kinases as targets for inhibition in neurofibromatosis type 2. Cancer research 68: 7932-7937, 2008.

245. Ying H, Kimmelman AC, Lyssiotis CA, Hua S, Chu GC, Fletcher-Sananikone E, Locasale JW, Son J, Zhang H, Coloff JL, Yan H, Wang W, Chen S, Viale A, Zheng H, Paik JH, Lim C, Guimaraes AR, Martin ES, Chang J, Hezel AF, Perry SR, Hu J, Gan B, Xiao Y, Asara JM, Weissleder R, Wang YA, Chin L, Cantley LC, and DePinho RA. Oncogenic Kras maintains pancreatic tumors through regulation of anabolic glucose metabolism. Cell 149: 656-670, 2012.

246. Yoshida S, Yokota T, Ujiki M, Ding XZ, Pelham C, Adrian TE, Talamonti MS, Bell RH, Jr., and Denham W. Pancreatic cancer stimulates pancreatic stellate cell proliferation and TIMP-1 production through the MAP kinase pathway. Biochemical and biophysical research communications 323: 1241-1245, 2004.

247. Yuen A and Diaz B. The impact of hypoxia in pancreatic cancer invasion and metastasis. Hypoxia 2: 91-106, 2014.

248. Zahnow CA and Baylin SB. Epigenetic networks and miRNAs in stem cells and cancer. Molecular cell 39: 661-663, 2010. 


\section{REFERENCES}

249. Zarubin T and Han J. Activation and signaling of the p38 MAP kinase pathway. Cell research 15: 11-18, 2005.

250. Zavoral M, Minarikova P, Zavada F, Salek C, and Minarik M. Molecular biology of pancreatic cancer. World journal of gastroenterology: WJG 17: 2897-2908, 2011.

251. Zhang J, Wang J, Guo Q, Wang Y, Zhou Y, Peng H, Cheng M, Zhao D, and Li F. LCH-7749944, a novel and potent p21-activated kinase 4 inhibitor, suppresses proliferation and invasion in human gastric cancer cells. Cancer letters 317: 24-32, 2012.

252. Zhang K, Chen D, Jiao X, Zhang S, Liu X, Cao J, Wu L, and Wang D. Slug enhances invasion ability of pancreatic cancer cells through upregulation of matrix metalloproteinase-9 and actin cytoskeleton remodeling. Laboratory investigation; a journal of technical methods and pathology 91: 426-438, 2011.

253. Zhao G, Wang B, Liu Y, Zhang JG, Deng SC, Qin Q, Tian K, Li X, Zhu S, Niu Y, Gong Q, and Wang CY. miRNA-141, Downregulated in Pancreatic Cancer, Inhibits Cell Proliferation and Invasion by Directly Targeting MAP4K4. Molecular cancer therapeutics 12: 2569-2580, 2013.

254. Zhao ZS, Lim JP, Ng YW, Lim L, and Manser E. The GIT-associated kinase PAK targets to the centrosome and regulates Aurora-A. Molecularcell 20: 237-249, 2005.

255. Zhao ZS and Manser E. Do PAKs make good drug targets? F1000 biology reports 2: 70, 2010.

256. Zhao ZS and Manser E. PAK family kinases: Physiological roles and regulation. Cellular logistics 2: 59-68, 2012.

257. Zhou J, Feng Y, Tao K, Su Z, Yu X, Zheng J, Zhang L, and Yang D. The expression and phosphorylation of ezrin and merlin in human pancreatic cancer. International journal of oncology 44: 2059-2067, 2014.

258. Zhou L, Yan C, Gieling RG, Kida Y, Garner W, Li W, and Han YP. Tumor necrosis factor-alpha induced expression of matrix metalloproteinase-9 through p21-activated kinase-1. BMC immunology 10: 15, 2009.

259. Zhou W, Jubb AM, Lyle K, Xiao Q, Ong CC, Desai R, Fu L, Gnad F, Song Q, Haverty PM, Aust D, Grutzmann R, Romero M, Totpal K, Neve RM, Yan Y, Forrest WF, Wang Y, Raja R, Pilarsky C, de Jesus-Acosta A, Belvin M, Friedman LS, Merchant M, Jaffee EM, Zheng L, Koeppen H, and Hoeflich KP. PAK1 mediates pancreatic cancer cell migration and resistance to MET inhibition. The Journal of pathology 234: 502-513, 2014.

260. Zhu G, Wang Y, Huang B, Liang J, Ding Y, Xu A, and Wu W. A Rac1/PAK1 cascade controls beta-catenin activation in colon cancer cells. Oncogene 31: 1001-1012, 2012. 


\section{REFERENCES}

261. Zhu L, Shi G, Schmidt CM, Hruban RH, and Konieczny SF. Acinar cells contribute to the molecular heterogeneity of pancreatic intraepithelial neoplasia. The American journal of pathology 171: 263-273, 2007.

262. Zhu M, Xu Z, Wang K, Wang N, and Li Y. microRNA and gene networks in human pancreatic cancer. Oncology letters 6: 1133-1139, 2013.

263. Zhu Y, Liu H, Xu L, An H, Liu W, Liu Y, Lin Z, and Xu J. p21-activated kinase 1 determines stem-like phenotype and sunitinib resistance via NF-kappaB/IL-6 activation in renal cell carcinoma. Cell death \& disease 6: e1637, 2015. 


\section{APPENDIX}

\section{Appendix}

\section{Appendix i. Mouse clinical signs severity score}

1. Category I clinical signs:

- Mandatory euthanasia should be performed if an animal scored 3 for any clinical signs from column 3.

- Separate from the condition above, the animals will be closely monitored or euthanised based on the total scoring system as given below.

\begin{tabular}{|l|l|}
\hline Total Score & Classification/Action \\
\hline $0-2$ & Good Health \\
\hline $3-8$ & Health to be monitored daily \\
\hline $9-12$ & $\begin{array}{l}\text { Poor Health - Observe animals twice daily, if no improvement within } 48 \\
\text { hours mandatory euthanasia }\end{array}$ \\
\hline 13 and above & Mandatory euthanasia \\
\hline
\end{tabular}

2. Category II clinical signs:

- Mandatory euthanasia should be performed if an animal scored 3 for any clinical sign from column 3 . This includes acute $(>10 \%)$ or chronic $(>15 \%)$ weight loss.

- Animals with clinical signs with a single score of 4 will be closely monitored (twice daily) and consider for euthanasia if the condition is deteriorating.

- Separate from the two conditions above, the animals will be closely monitored or euthanised based on the total scoring system as given below.

\begin{tabular}{|l|l|}
\hline Total Score & Classification/Action \\
\hline $0-2$ & Good Health \\
\hline $3-8$ & Health to be monitored daily \\
\hline $9-12$ & $\begin{array}{l}\text { Poor Health - Observe animals twice daily, if no improvement within } 48 \\
\text { hours mandatory euthanasia }\end{array}$ \\
\hline 13 and above & Mandatory euthanasia \\
\hline
\end{tabular}


CLINICAL SIGNS SEVERITY SCORE - Category I (1-3)

\begin{tabular}{|c|c|c|c|c|}
\hline SIGNS & $\mathbf{0}$ & 1 & 2 & 3 \\
\hline Activity & normal & $\begin{array}{l}\text { isolated, } \\
\text { abnormal } \\
\text { posture }\end{array}$ & $\begin{array}{l}\text { huddled/inactive } \\
\text { OR overactive }\end{array}$ & $\begin{array}{l}\text { moribund } \\
\text { OR fitting }\end{array}$ \\
\hline Alertness/Sleeping & normal & $\begin{array}{l}\text { dull or } \\
\text { depressed }\end{array}$ & $\begin{array}{l}\text { little response to } \\
\text { handling }\end{array}$ & unconscious \\
\hline Coat & normal & coat rough & $\begin{array}{l}\text { unkempt; wounds, hair } \\
\text { thinning }\end{array}$ & $\begin{array}{l}\text { bleeding or infected } \\
\text { wounds, or severe hairloss } \\
\text { or self mutilation }\end{array}$ \\
\hline Faeces & normal & faeces moist & loose, soiled perineum & $\begin{array}{l}\text { Presence of diarrhoea for } 48 \\
\text { hours OR no faeces for } 48 \\
\text { hrs OR presence ofblood on } \\
\text { faeces }\end{array}$ \\
\hline $\begin{array}{l}\text { Movement/ } \\
\text { gait }\end{array}$ & normal & $\begin{array}{l}\text { slight OR } \\
\text { abnormal } \\
\text { gait }\end{array}$ & $\begin{array}{l}\text { walking on tiptoe } \mathrm{OR} \\
\text { reluctance to move }\end{array}$ & $\begin{array}{l}\text { staggering } \text { OR limb } \\
\text { dragging OR paralysis }\end{array}$ \\
\hline Nose & normal & wetness & discharge & $\begin{array}{l}\text { Obstruction of nasal } \\
\text { passages or constant } \\
\text { purulent discharge }\end{array}$ \\
\hline Vocalisation & normal & $\begin{array}{l}\text { squeaks } \\
\text { when } \\
\text { palpated }\end{array}$ & $\begin{array}{l}\text { struggles and squeaks } \\
\text { loudly in response to } \\
\text { handling of a body part } \\
\text { \& signs of aggression }\end{array}$ & $\begin{array}{l}\text { persistent and } \begin{array}{r}\text { repetitive } \\
\text { vocalisation } \\
\text { handling }\end{array} \\
\text { without } \\
\end{array}$ \\
\hline $\begin{array}{ll}\text { Surgical } & \text { site } \\
\text { appearance } & \end{array}$ & $\begin{array}{l}\text { normal (no } \\
\text { swelling or } \\
\text { redness) }\end{array}$ & $\begin{array}{l}\text { mild } \\
\text { redness, } \\
\text { mild } \\
\text { swelling of } \\
\text { wound }\end{array}$ & $\begin{array}{l}\text { Presence of discharge } \\
\text { and presence of } \\
\text { moderate swelling }\end{array}$ & $\begin{array}{l}\text { redness and swelling of the } \\
\text { wound OR loss of wound } \\
\text { clip and/or separation of } \\
\text { wound edges. *Euthanasia } \\
\text { if re-suturing and/or } \\
\text { surgical intervention fails }\end{array}$ \\
\hline
\end{tabular}

CLINICAL SIGNS SEVERITY SCORE - Category II (1-3)

\begin{tabular}{|c|c|c|c|c|}
\hline SIGNS & $\mathbf{0}$ & 1 & 2 & 3 \\
\hline Body condition & normal & thin & $\begin{array}{l}\text { loss of body fat, failure } \\
\text { to grow }\end{array}$ & $\begin{array}{l}\text { loss of muscle mass that } \\
\text { enables palpation of the } \\
\text { spine }\end{array}$ \\
\hline Body weight & $\begin{array}{l}\text { normal } \\
\text { weight } \\
\text { and } \\
\text { growth } \\
\text { rate }\end{array}$ & $\begin{array}{l}\text { reduced growth } \\
\text { rate }\end{array}$ & $\begin{array}{l}\text { Chronic weight loss } \\
>10 \% * * \text { OR failure to } \\
\text { grow }\end{array}$ & $\begin{array}{l}\text { acute weight loss }>10 \% * \\
\text { chronic weight loss } 15 \% * * \\
\text { OR failure to grow for } \\
\text { juveniles or failure to } \\
\text { stabilise body weight }\end{array}$ \\
\hline Breathing & normal & rapid, shallow & $\begin{array}{l}\text { rapid, abdominal } \\
\text { breathing }\end{array}$ & $\begin{array}{l}\text { laboured, irregular, or } \\
\text { gaping mouth breaking, } \\
\text { and/or blue skin }\end{array}$ \\
\hline Dehydration & none & skin less elastic & skin tenting & skin tenting \& eyes sunken \\
\hline Drinking & normal & $\begin{array}{l}\text { increased OR } \\
\text { decreased intake } \\
\text { over } 24 \mathrm{hrs} \\
\end{array}$ & $\begin{array}{l}\text { increased OR decreased } \\
\text { intake over } 48 \text { hours }\end{array}$ & $\begin{array}{l}\text { constantly drinking OR } \\
\text { not drinking over } 24 \text { hours }\end{array}$ \\
\hline Eating & normal & $\begin{array}{l}\text { increased OR } \\
\text { decreased intake } \\
\text { over } 24 \text { hours } \\
\end{array}$ & $\begin{array}{l}\text { increased OR decreased } \\
\text { intake over } 48 \text { hours }\end{array}$ & $\begin{array}{l}\text { obese OR not eating over } \\
48 \text { hours }\end{array}$ \\
\hline Eyes & normal & $\begin{array}{l}\text { wetness } \\
\text { dullness }\end{array}$ & discharge & Vision obstructed \\
\hline Other & & & & \\
\hline
\end{tabular}

*, Acute refers to the weight loss occurring within 2 days.

**, Chronic refers to the weight loss occurring within 7 days; 


\section{University Library}

\section{- M I I N E R VA \\ A gateway to Melbourne's research publications}

Minerva Access is the Institutional Repository of The University of Melbourne

Author/s:

Yeo, Dannel

Title:

Role of p21-activated kinases in pancreatic cancer

Date:

2016

Persistent Link:

http://hdl.handle.net/11343/142355

Terms and Conditions:

Terms and Conditions: Copyright in works deposited in Minerva Access is retained by the copyright owner. The work may not be altered without permission from the copyright owner. Readers may only download, print and save electronic copies of whole works for their own personal non-commercial use. Any use that exceeds these limits requires permission from the copyright owner. Attribution is essential when quoting or paraphrasing from these works. 